\title{
Top Quark Pair Production Cross Section in the Lepton+Jets Channel Using b-tagging at DØ
}

\author{
by \\ Hwidong Yoo \\ B.S., Yonsei University, Republic of Korea 1997 \\ M.S., Yonsei University, Republic of Korea 2001 \\ Sc.M., Brown University, 2004
}

Thesis

Submitted in partial fulfillment of the requirements for the Degree of Doctor of Philosophy

in the Department of Physics at Brown University

Providence, Rhode Island

May 2008 
Abstract of " Top Quark Pair Production Cross Section in the Lepton+Jets Channel Using b-tagging at DØ" by Hwidong Yoo, Ph.D., Brown University, May 2008

The top quark pair production cross section measurement in the lepton+jets channel with b-tagging algorithm is described. About $900 \mathrm{pb}^{-1}$ data collected by the $\mathrm{D} \varnothing$ detector at the Fermilab Tevatron are used for this analysis. In this thesis, event selection, background estimation, and cross section calculation are discussed in detail. In addition, calibration of the Luminosity Monitor readout electronics and a new b-tagging algorithm, the SLTNN tagger, are also discussed in this thesis. 
(c) Copyright

by

Hwidong Yoo

2008 
This dissertation by Hwidong Yoo is accepted in its present form by the Department of Physics as satisfying the dissertation requirement for the degree of Doctor of Philosophy

Date.......................

\section{Richard Partridge, Advisor}

Recommended to the Graduate Council

Date.......................

David Lowe, reader

Date.......................

Meenakshi Narain, reader

Approved by the Graduate Council

Date.......................

Sheila Bonde

Dean of the Graduate School 
The Vita of Hwidong Yoo

Hwidong Yoo was born in Seoul, Republic of Korea on August $4^{\text {th }} 1974$. He graduated with B.S. in Physics Department from Yonsei University at 1997. He served in Army (Republic of Korea) from 1997 to 1999 during 28 months. He graduated with M.S. in Physics Department from Yonsei University at 2001. After receiving M.S., he moved to USA and graduated with Sc.M. in Physics Department from Brown University at 2004. 


\section{Preface and Acknowledgments}

First of all, I would like to thank my advisor, Professor Richard Partridge. Rich was always patient for my poor English expression and taught me knowledge of both detector electronics and physics analysis. He encouraged and guided me to solve a problem and make a progress. Without his support, this dissertation would not have been possible. I will always keep in mind his advice. I would also like to thank Professor David Lowe and Professor Meenakshi Narain for accepting committee members of my thesis defense and a cafeful reading of my thesis.

I am particularly grateful to Professor Meenakshi Narain, Professor Ulrich Heintz and Dr. Dookee

Cho for their help in $t \bar{t}$ cross section analysis. I was very happy to work with them and lucky to learn all aspects of top quark physics as well as physics analysis from them, although I could not sleep sometimes.

In addition, I am highly thankful to members of Brown HEP group. Especially, I was very delightful to work with Dr. Brendan Casey and Dr. Yuji Enari of Brown Luminosity Monitor Group. I learned a lot of things for hardware from Brendan and his help was very crucial to the success of the LM project. Brendan and Yuji gave me very good advices about sensitive questions.

I had many experiences with my Korean friends at Fermilab and I was very enjoyful with them. I wish them endless successes in their HEP careers. I am also thankful to my friends outside DØ. Although I could not meet them frequently, I was encouraged by the fact that they believe and support me always.

I deeply appreciate my family for their love and support. I always keep in mind their unconditional love. Especially my wife, Mihea Jung, supports and believes me for everything. I deeply thank to my wife and her love.

Finally, I would like to dedicate this thesis to my parents who give me eternal love in my life. 


\section{Contents}

1 Introduction 1

1.1 Standard Model . . . . . . . . . . . . . . . . . . . . . . . . . 1

1.2 Top Quark Pair Production and Decay . . . . . . . . . . . . . . . . . 4

1.2.1 Total Cross Section for the Top Quark Pair Production . . . . . . . . . 5

1.2 .2 Top Quark Decay. . . . . . . . . . . . . . . . . . . . . . 6

2 Tevatron and DØ Detector 10

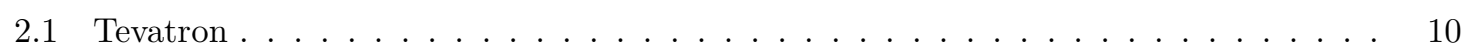

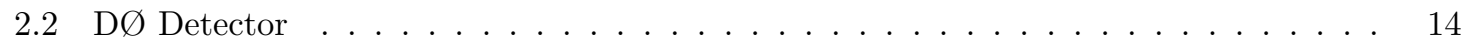

2.2 .1 Coordinate System . . . . . . . . . . . . . . . . . . . . . . 15

2.2 .2 Central Tracking System . . . . . . . . . . . . . . . . . 15

2.2 .3 Calorimeter . . . . . . . . . . . . . . . . . . . 17

2.2 .4 Muon Detector . . . . . . . . . . . . . . . . . . . 20

2.2 .5 Luminosity Monitor . . . . . . . . . . . . . . . . . . . . . 20 


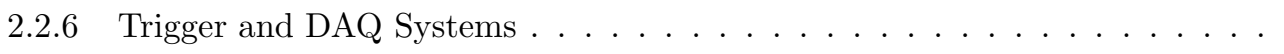

3 Event Reconstruction and Monte Carlo Simulation $\quad 25$

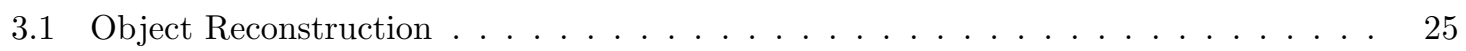

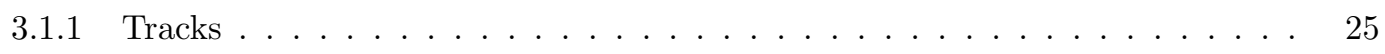

$3.1 .2 \quad$ Primary Vertices $\ldots \ldots \ldots \ldots \ldots \ldots \ldots \ldots \ldots \ldots$

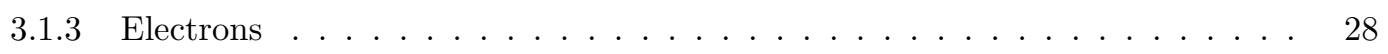

3.1 .4 Muons . . . . . . . . . . . . . . . . . . . . . . . . . . . . . 29

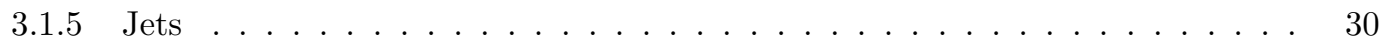

3.1.6 Missing Transverse Energy _ . . . . . . . . . . . . . . . . . 35

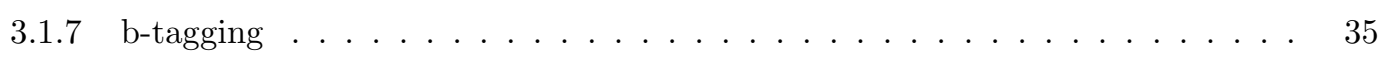

3.2 Monte Carlo Simulation . . . . . . . . . . . . . . . . . . . 36

$3.2 .1 \quad$ Monte Carlo Generation at $\mathrm{D} \emptyset \ldots \ldots \ldots \ldots \ldots$

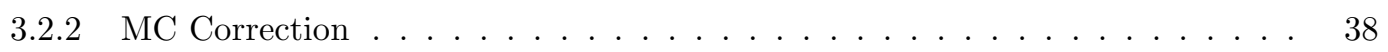

4 Data Sample and Event Selection $\quad 45$

4.1 Data Sample . . . . . . . . . . . . . . . . . . . 45

4.2 Monte Carlo Samples . . . . . . . . . . . . . . . . . . . . . 46

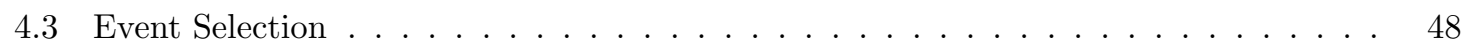

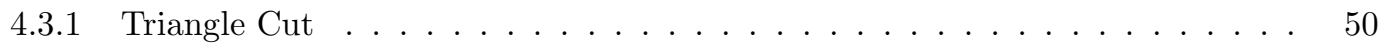

4.3.2 Event Selection Efficiency for $t \bar{t}$ MC Sample . . . . . . . . . . . . . . 50

4.4 b-tagging Optimization $\ldots \ldots \ldots \ldots \ldots \ldots \ldots \ldots$

$\begin{array}{llr}5 & \text { Background Estimation } & 58\end{array}$

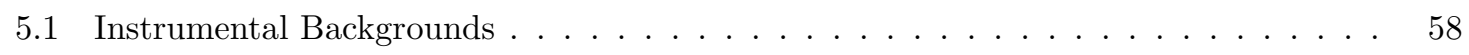

5.2 Physics Backgrounds . . . . . . . . . . . . . . . . . . . 62

5.3 Background Estimation in b-tagged Events . . . . . . . . . . . . 65

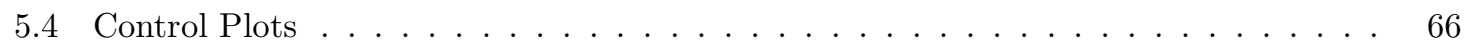




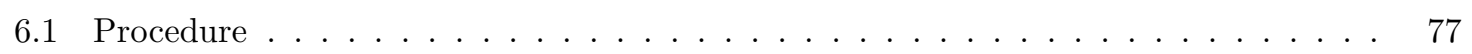

6.2 Cross Section with Statistical Uncertainty . . . . . . . . . . . . . . . 83

6.3 Systematic Uncertainties . . . . . . . . . . . . . . . . . . . . . . . . . 84

6.3.1 Uncertainties on the Preselection Efficiency . . . . . . . . . . . 86

6.3.2 Uncertainties on Both Preselection Efficiency and Tagging Probability . . . . 88

6.3.3 Summary of Systematic Uncertainties . . . . . . . . . . . . . . . . 90

6.4 Results................................. . . . . . 90

$\begin{array}{llr}7 & \text { Conclusion } & 96\end{array}$

$\begin{array}{lr}\text { Appendix } & 98\end{array}$

A Calibration of the Luminosity Monitor Readout Electronics 98

A.1 LM Readout Electronics . . . . . . . . . . . . . . . . . . . . . . . . . . . 98

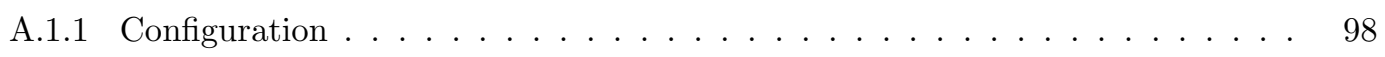

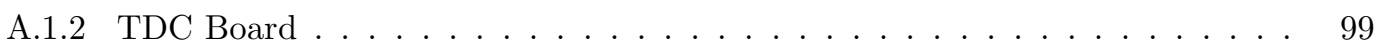

A.1.3 VTX Board . . . . . . . . . . . . . . . . . . . 102

A.2 Calibration .......................... . . . 106

A.2.1 CAFÉ Card Calibration ...................... 106

A.2.2 Global Time Calibration . . . . . . . . . . . . . . . . . . 110

B Soft Lepton Tagger with Neural Network Method (SLTNN tagger) 113

B.1 Introduction . . . . . . . . . . . . . . . . . . . . . . . . 113

B.1.1 Samples . . . . . . . . . . . . . . . . . . . 114

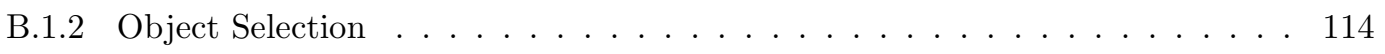

B.1.3 Method Description ..................... 115

B.2 Neural Network Method . . . . . . . . . . . . . . . . . . . . . 116

B.3 Tagging Efficiency . . . . . . . . . . . . . . . . . . . . . . . . . 123

B.3.1 Muon Tagging Efficiency in the MC Samples . . . . . . . . . . . . . . . 123 
B.3.2 System8 Method . . . . . . . . . . . . . . . . . . . . . . . . . . . 124

B.3.3 The Results for Tagging Efficiencies _ . . . . . . . . . . . . . . . . . . 134

B.3.4 Systematic Uncertainties . . . . . . . . . . . . . . . . . . . . . . 135

B.4 Fake Rate . . . . . . . . . . . . . . . . . . . . . . . . . . . . . . . . . 139

B.5 $t \bar{t}$ Cross Section Measurement Using the SLTNN Tagging . . . . . . . . . . . . 141

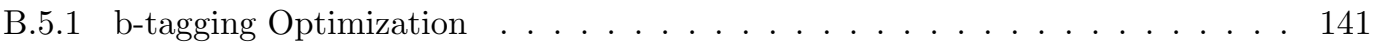

B.5.2 Control Plots . . . . . . . . . . . . . . . . . . . . . . . . . . 142

B.5.3 Cross Section Calculation . . . . . . . . . . . . . . . . . . . . . . 147

B.5.4 Systematic Uncertainty . . . . . . . . . . . . . . . . . . . . . . 149

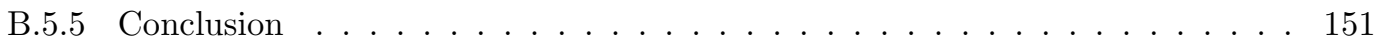

$\begin{array}{ll}\text { C Control Plots } & 152\end{array}$

C.1 Control Plots for the $e+$ jets Channel . . . . . . . . . . . . . . . . . . 153

C.1.1 Preselection Events . . . . . . . . . . . . . . . . . . . . . . 153

C.1.2 Exactly One b-tagged Events . . . . . . . . . . . . . . . . . 156

C.1.3 Two or More b-tagged Events . . . . . . . . . . . . . . . . . . . . . . 161

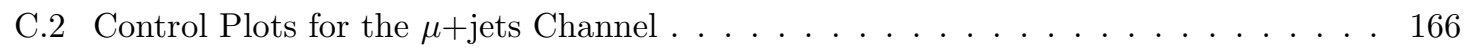

C.2.1 Preselection Events . . . . . . . . . . . . . . . . . . . . . 166

C.2.2 Exactly One b-tagged Events . . . . . . . . . . . . . . . . . . 169

C.2.3 Two or More b-tagged Events . . . . . . . . . . . . . . . . . . . . . . . 174

$\begin{array}{lr}\text { Bibliography } & 179\end{array}$ 


\section{List of Tables}

1.1 The summary of characteristics for quarks and leptons. . . . . . . . . . . . . 2

1.2 Summary of the four fundamental forces and their gauge bosons [4]. . . . . . . . . 3

1.3 The $t \bar{t}$ pair production cross section in $p \bar{p}$ collision at Tevatron $(\sqrt{s}=1.96 \mathrm{TeV})$ for $m_{t}=175 \mathrm{GeV}$ [7]. MRST2002 [9] and CTEQ6M [10] are parton distribution functions. 1PI denotes single-particle-inclusive kinematics and PIM denotes pairinvariant-mass kinematics. $\ldots \ldots \ldots \ldots \ldots \ldots \ldots$

1.4 The $t \bar{t}$ cross section predictions for the CTEQ6M and MRST parton distribution functions at $\sqrt{s}=1.96 \mathrm{TeV}[8] . \ldots \ldots \ldots \ldots \ldots \ldots$

1.5 The branching fractions of the $\mathrm{W}$ boson decay mode $[3] . \ldots \ldots \ldots \ldots$

3.1 NN input variables ranked in order of power $[34] \ldots \ldots \ldots \ldots \ldots$

4.1 Integrated luminosity for the triggers used in the $e+$ jets channel. . . . . . . . 45

4.2 Integrated luminosity for the triggers used in the $\mu+$ jets channel. $\ldots \ldots \ldots \ldots$

4.3 Summary of the $t \bar{t} \rightarrow e+j e t s$ event preselection efficiencies and the corresponding scale factors $(\kappa)$, as applicable, for exactly three jets. Only statistical uncertainties are included. . . . . . . . . . . . . . . . . . . . .

4.4 Summary of the $t \bar{t} \rightarrow e+$ jets event preselection efficiencies and the corresponding scale factors $(\kappa)$, as applicable, for four or more jets. Only statistical uncertainties

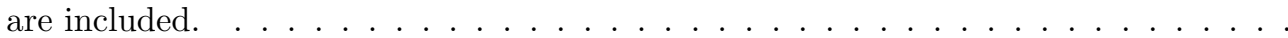


4.5 Summary of the $t \bar{t} \rightarrow \mu+$ jets event preselection efficiencies and the corresponding scale factors $(\kappa)$, as applicable, for exactly three jets. Only statistical uncertainties are included. . . . . . . . . . . . . . . . . . . . . .

4.6 Summary of the $t \bar{t} \rightarrow \mu+$ jets event preselection efficiencies and the corresponding scale factors $(\kappa)$, as applicable, for four or more jets. Only statistical uncertainties

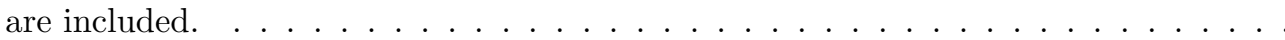

4.7 Preselection efficiency for $t \bar{t}$ dilepton MC samples. The error is statistical only. . . . 55

4.8 The NN tagger's operating points [34] $\ldots \ldots \ldots \ldots \ldots \ldots \ldots$

4.9 Signal and background ratios of different operating points in the $e+$ jets channel. The best operating point has been chosen to maximize $S / \sqrt{S+B}$ for the third inclusive

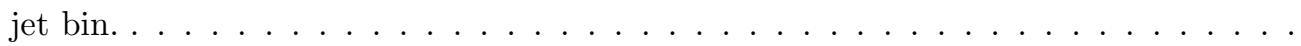

4.10 Signal and background ratios of different operating points in the $\mu+$ jets channel. The best operating point has been chosen to maximize $S / \sqrt{S+B}$ for the third inclusive

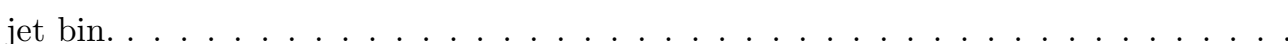

$5.1 \varepsilon_{s i g}$ for different jet multiplicities in both channels. $\varepsilon_{Q C D}$ is measured without a jet multiplicity dependence. The first error is the statistical error and the second one is the systematic error $(\%) \ldots \ldots \ldots \ldots \ldots$

5.2 Number of preselected events in the loose and tight samples. The expected contribution from multijet and $W$-like events are obtained by the matrix method for different jet multiplicity bins in the $e+$ jets channel. . . . . . . . . . . .

5.3 Number of preselected events in the loose and tight samples. The expected contribution from multijet and $W$-like events are obtained by the matrix method for different jet multiplicity bins in the $\mu+$ jets channel. . . . . . . . . . . . . . . .

5.4 Summary of event yields before $b$-tagging in the $e+$ jets channel. . . . . . . . . . . . 64

5.5 Summary of event yields before $b$-tagging in the $\mu+$ jets channel. . . . . . . . . . 65

5.6 Summary of event yields with exactly one $b$-tag in the $e+$ jets channel. . . . . . . 67

5.7 Summary of event yields with exactly one $b$-tag in the $\mu+$ jets channel. . . . . . . . 67 
5.8 Summary of event yields with two or more $b$-tags in the $e+$ jets channel. . . . . . . 68

5.9 Summary of event yields with two or more $b$-tags in the $\mu+$ jets channel. . . . . . . 68

6.1 The $t \bar{t}$ cross section results for each channel. The errors are statistical only. $\quad \ldots . \quad 84$

6.2 The combined results of cross section. The errors are statistical only. . . . . . . . 84

6.3 Summary of the systematic uncertainties. The notation "uncorrelated" and "correlated" refer to the treatment of the sources of errors in the cross section combination.

6.4 Summary of the total systematic uncertainties for the combined results (exactly one

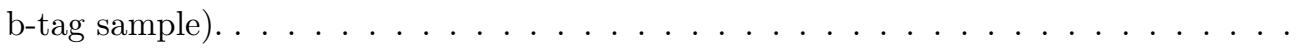

6.5 Summary of the total systematic uncertainties for the combined results (two or more b-tags sample) $\ldots \ldots \ldots \ldots \ldots \ldots \ldots \ldots \ldots \ldots \ldots \ldots \ldots \ldots \ldots \ldots$

6.6 Summary of the total systematic uncertainties for the combined results (all channels). 95

A.1 The operation on four capacitors in each stage. $\ldots \ldots \ldots \ldots \ldots$

A.2 The lookup table address, 16 bits. 10 bits of 16 bits are ADC output. Range is the information from the QIE and the CapID is the capacitor ID of the QIE. H/L decides a mode of "pass-through" or "calibrated". . . . . . . . . . . . . . . . . 108

A.3 The data format used to store the CAFÉ card calibration results [72] . . . . . . . 110

A.4 The data format used to store the global time calibration results [72]. . . . . . . . 111

A.5 Format of the Time CAFÉ lookup table output. . . . . . . . . . . . . . . . . 112

A.6 Format of the Charge CAFÉ lookup table output. . . . . . . . . . . . . . . . 112

B.1 The data samples used in this analysis. . . . . . . . . . . . . . . 114

B.2 MC samples which are used in this study $\ldots \ldots \ldots \ldots \ldots \ldots \ldots$

B.3 Muon definitions used in this analysis f . . . . . . . . . . . . . 115

B.4 NN input variables and descriptions. The SL denotes the SuperLoose operating point

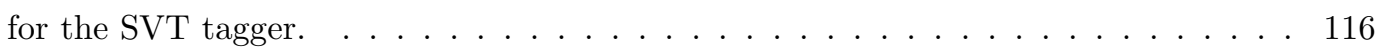

B.5 The NN cuts for the operating points for each muon definition. . . . . . . . . . 123

B.6 Summary of correlation coefficients with statistical uncertainties. . . . . . . . . . . 134 
B.7 b-tagging efficiency measured by the system8 method for the MUinclusive sample for each muon definition and its $\mathrm{NN}$ operating points. . . . . . . . . . . . . . . 135

B.8 Estimated systematic uncertainties for $S F, T R F_{b}$, and $T R F_{c}$. The systematic uncertainties estimated by the system 8 method and closure tests are also shown in this

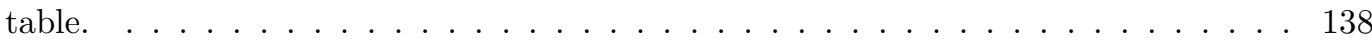

B.9 Fake rate measured in the overall QCD data sample (without any jet dependence). 140

B.10 Systematic uncertainty in the fake rate for CC, ICR and EC regions. $\quad \ldots \ldots \ldots \ldots 141$

B.11 The results of $S / \sqrt{S+B}$ ratio for each operating point. The best operating point is the Loose NN operating point with the loose muon definition. . . . . . . . . . . . 142

B.12 Summary of event yields for the $e+$ jets channel after SLTNN tagging. . . . . . . . . 147

B.13 Summary of event yields for the $\mu+$ jets channel after SLTNN tagging. . . . . . . . 148

B.14 Summary of total systematic unertainties for combined results (all channels). . . . . 150 


\section{List of Figures}

1.1 The lowest order processes for $t \bar{t}$ pair production in QCD [5]. . . . . . . . . . 4

1.2 The $t \bar{t}$ cross section in $p \bar{p}$ collision at $\sqrt{S}=1.96 \mathrm{TeV}$ as a function of $m_{t}$ for $\mu=m_{t}$. The NLO (solid) and NNLO (dotted) with uncertainties (dashed and dot-dashed) [7]. 6

1.3 Decay channels for $t \bar{t}$ pair events and their branching fractions [5]. . . . . . . . 8

1.4 The event signature of $t \bar{t}$ pair production in the lepton+jets channel $[5] \ldots \ldots$

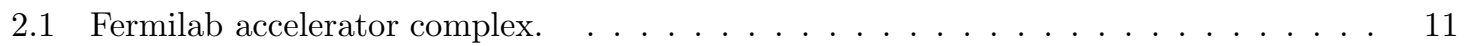

2.2 Diagram of the LINAC which shows how $\mathrm{H}^{-}$ions are accelerated $[14] . \ldots \ldots$

2.3 The diagram of the system used to accumulate and cool the antiprotons [15]. . . . 12

2.4 Luminosity at the Tevatron during Run II (2001-2008) [17]. . . . . . . . . . . . 13

2.5 Diagram of the upgraded DØ detector viewed from inside the Tevatron ring. . . . . 14

2.6 Cross-sectional view of the central tracking system with solenoid in the $x-z$ plane. $\quad 16$

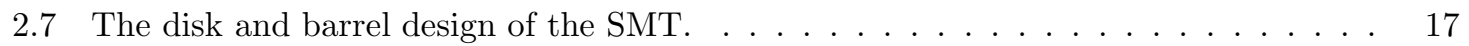

2.8 Isometric view of the central and two end calorimeters. $\ldots \ldots \ldots \ldots \ldots$

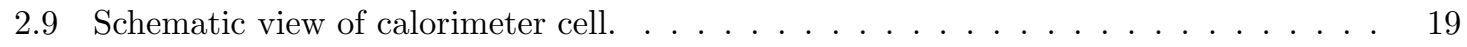

2.10 Schematic view of the $\mathrm{D} \varnothing$ calorimeter. The rays and the numbers indicate pseudorapidity intervals seen from the center of detector. . . . . . . . . . . . . . 19

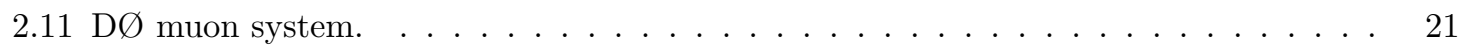

2.12 Schematic view of the LM detectors. . . . . . . . . . . . . . . . . 22

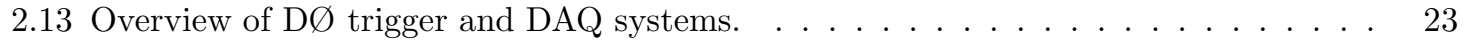

2.14 Block diagram of the L1 and L2 trigger systems. The arrows show the data flow. $\quad 24$ 
3.1 Illustration for an example of Hough transformation. This example is for $1.5 \mathrm{GeV}$ muon track. (a) The family of trajectories containing a given hit. (b) The geometric place of all trajectories containing a given hit in parameter space. (c) Curves from different hits intersect at one point corresponding to the track parameters. (d) The point of intersection can be seen as a peak in the $(\rho, \phi)$ histogram [19]. . . . . . . 26

3.2 Offset energy of $\Delta R<0.5$ cone jets for various primary vertex multiplicites [28]. . . 32

3.3 The MPF method description $[28] . \ldots \ldots \ldots \ldots \ldots \ldots \ldots \ldots$

3.4 The measured absolute response results for various $\eta$ regions as a function of partially corrected jet energy $[28] . \ldots \ldots \ldots \ldots \ldots \ldots \ldots \ldots \ldots \ldots \ldots \ldots \ldots \ldots \ldots$

3.5 The measured showering correction for $\Delta R<0.5$ jet cone in data as a function of corrected jet $E_{\mathrm{T}}[28] . \ldots \ldots \ldots \ldots \ldots \ldots \ldots \ldots \ldots \ldots \ldots \ldots \ldots \ldots \ldots \ldots$

3.6 Efficiency vs. Fake rate plot for the NN tagger and the JLIP tagger [34] . . . . . . 37

3.7 A MC event with $50 \mathrm{GeV} p_{\mathrm{T}}$ particles simulated in d0gstar [43] . . . . . . . . 38

3.8 Electron reconstruction efficiency for data (red) and MC (blue) $Z \rightarrow e e$ samples in (a) top. Bottom plot of (a) is the scale factor for electron reconstruction. Plots in (b) show the track matching and liklihood cut efficiencies (top) of data (red) and MC (blue) as well as the scale factor (bottom) $[23] \ldots \ldots \ldots \ldots$

3.9 Ratio of data and MC efficiencies for muon reconstruction (top), muon track-matching (middle) and muon isolation (bottom) [24]. In the bottom plot, the green is the scale

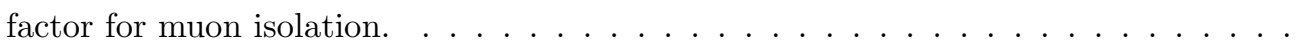

3.10 Distributions of $\langle\Delta S\rangle_{\text {data }}-\langle\Delta S\rangle_{M C}$ before (left) and after (right) applying corrections to the MC jets in CC (top), ICR (middle), and EC (bottom) $\eta$ region [44].

3.11 b-tagging efficiency in MC (red line) and data (green line) and the scale factor between them (blue line) with $1 \sigma$ error bands (black dashed line) on jet $p_{\mathrm{T}}$ (left) and $\eta$ (right)

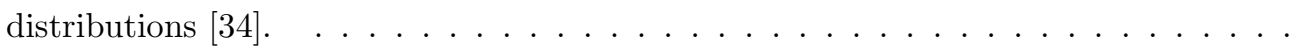

3.12 b-tagging efficiency in MC (red line) and TRF for b-jets (green line) with $1 \sigma$ error bands (black dashed line) on jet $p_{\mathrm{T}}$ (left) and $\eta$ (right) distributions [34]. . . . . . . 
4.1 Leading jet $p_{\mathrm{T}}$ (left) and $\mathbb{E}_{T}$ distributions for $t \bar{t}$ signal events (MC) and multijet background events (data) in the $e+$ jets channel. The black solid line is for $t \bar{t}$ events and the red dotted line is for multijet events. The blue dot-dashed line is the leading jet $p_{\mathrm{T}}$ cut (left) and the $E_{T}$ cut (right) used in this analysis. The y-axis is normalized to 1 .

4.2 Leading jet $p_{\mathrm{T}}$ (left) and $E_{T}$ distributions for $t \bar{t}$ signal events (MC) and multijet background events (data) in the $\mu+$ jets channel. The black solid line is for $t \bar{t}$ events and the red dotted line is for multijet events. The blue dot-dashed line is the leading jet $p_{\mathrm{T}}$ cut (left) and the $E_{T}$ cut (right) used in this analysis. The y-axis is normalized to 1.

4.3 $\Delta \phi\left(e, E_{T}\right)$ vs. $E_{T}$ distributions for multijet events in the $e+$ jets channel. Jet multiplicities in the $1,2,3, \geq 4$ bins are shown as follows 1 jet (top left), 2 jets (top right), 3 jets (bottom left), and $\geq 4$ jets (bottom right) $[58] . \ldots \ldots \ldots$

$4.4 \Delta \phi\left(\mu, E_{T}\right)$ vs. $\mathbb{E}_{T}$ distributions for multijet events in the $\mu+$ jets channel. Jet multiplicities in the $1,2,3, \geq 4$ bins are shown as follows 1 jet (top left), 2 jets (top right), 3 jets (bottom left), and $\geq 4$ jets (bottom right) $[58] \ldots \ldots \ldots$

5.1 Electron $p_{\mathrm{T}}$ distributions for the preselected sample in the $e+$ jets channel. The plots show the results for different jet multiplicities: $=1$ jet (top left), $=2$ jets (top right), $=3$ jets $($ bottom left $)$, and $\geq 4$ jets $($ bottom right $) . \ldots \ldots \ldots$

5.2 Muon $p_{\mathrm{T}}$ distributions for the preselected sample in the $\mu+$ jets channel. The plots show the results for different jet multiplicities: $=1$ jet (top left), $=2$ jets (top right), $=3$ jets (bottom left), and $\geq 4$ jets (bottom right).

5.3 $E_{T}$ distributions for the preselected sample in the $e+$ jets channel. The plots show the results for different jet multiplicities: $=1$ jet (top left), $=2$ jets (top right), $=3$ jets

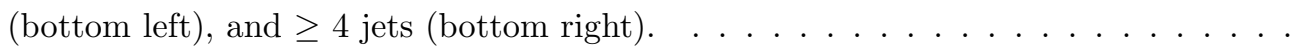


$5.4 \quad E_{T}$ distributions for the preselected sample in the $\mu+$ jets channel. The plots show the results for different jet multiplicities: $=1$ jet (top left), $=2$ jets (top right), $=3$

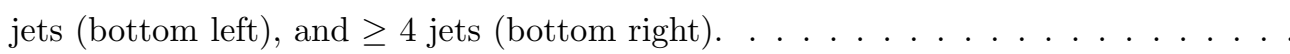

5.5 Leading jet $p_{\mathrm{T}}$ distributions for the preselected sample in the $e+$ jets channel. The plots show the results for different jet multiplicities: $=1$ jet (top left), $=2$ jets (top right), $=3$ jets (bottom left), and $\geq 4$ jets (bottom right). $\ldots \ldots \ldots$

5.6 Leading jet $p_{\mathrm{T}}$ distributions for the preselected sample in the $\mu+$ jets channel. The plots show the results for different jet multiplicities: $=1$ jet (top left), $=2$ jets (top

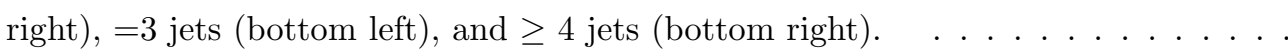

5.7 The $e \nu$ transverse mass distributions for the preselected sample in the $e+$ jets channel. The plots show the results for different jet multiplicities: $=1$ jet (top left), $=2$ jets

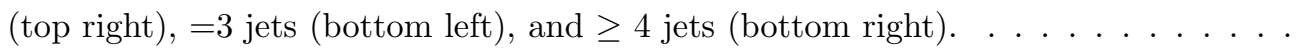

5.8 The $\mu \nu$ transverse mass distributions for the preselected sample in the $\mu+$ jets channel. The plots show the results for different jet multiplicities: $=1$ jet (top left), $=2$ jets (top right), $=3$ jets $($ bottom left), and $\geq 4$ jets $($ bottom right $) \ldots \ldots \ldots$

6.1 The predicted signal and background compositions for exactly one tag (left) and two or more tags (right) samples in the $e+$ jets channel with the measured $t \bar{t}$ cross section. The combined cross section result for all channels $(8.05 \mathrm{pb})$ is used in the figure. The red area is the contribution of $t \bar{t} \rightarrow l+$ jets signal events and the shaded region in the plots indicates the statistical uncertainty in the predicted number of events. . . . .

6.2 The predicted signal and background compositions for exactly one tag (left) and two or more tags (right) samples in the $\mu+$ jets channel with the measured $t \bar{t}$ cross section. The combined cross section result for all channels $(8.05 \mathrm{pb})$ is used in the figure. The red area is the contribution of $t \bar{t} \rightarrow l+$ jets signal events and the shaded region in the plots indicates the statistical uncertainty in the predicted number of events. . . . . 
6.3 The predicted signal and background compositions for exactly one tag (left) and two or more tags (right) samples in the lepton+jets channel with the measured $t \bar{t}$ cross section. The combined cross section result for all channels $(8.05 \mathrm{pb})$ is used in the figure. The red area is the contribution of $t \bar{t} \rightarrow l+$ jets signal events and the shaded region in the plots indicates the statistical uncertainty in the predicted number of

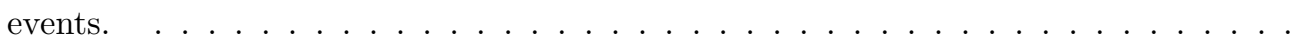

6.4 The measured top quark pair production cross section as a function of the top quark mass. The two green lines are the theoretical cross sections calculated by Cacciari et al. and Kidonakis and Vogt. The green (blue) dotted lines denote the uncertainties in the cross section The world average top quark mass at present, predictions (the measurement in this analysis). $172.6 \pm 0.8$ (stat) \pm 1.1 (sys) [11], is also shown in this

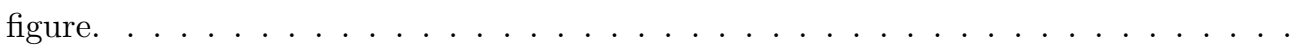

7.1 Summary of selected top quark pair production cross section measurements made by the CDF and DØ. The shaded area is the theoretical cross section with its uncertainty. 97

A.1 Overview of the LM readout system $[69] \ldots \ldots \ldots \ldots \ldots$

A.2 Photograph of the LM VME crate. The PowerPC, SBC, MFC, three LM TDC boards for the north $(-\mathrm{z})$ detector, LM VTX board, and three LM TDC boards for the south $(+z)$ LM detector are shown from left to right $[69] . \ldots \ldots \ldots$

A.3 Photograph of the TDC board $[69] . \ldots \ldots \ldots \ldots \ldots$

A.4 Photograph of the VTX board [69]. The mounted circuit is the mezzanine card with the FPGA. . . . . . . . . . . . . . . . . . . . . . 104

A.5 High-level block diagram of the VTX board showing the flow of major signals. Signals are generally labeled near their source. In some cases, only a subset of the bus lines may be connected to a particular element $[71] \ldots \ldots \ldots$. . . . . . . . 105

A.6 ADC vs. VCAL taken from CAFÉ card for calibration. . . . . . . . . . . . . . 107 
A.7 Cartoon of the lookup table. Data flows from left to right. Mode $=1$ is "calibrated" and mode $=0$ is "pass-through" $[73] . \ldots \ldots \ldots \ldots \ldots$

A.8 A comparsion between the true integrated charge (x-axis) and the calibrated charge (y-axis) for all of the ranges in a CAFÉ card. It is observed to be almost linear. . . 109

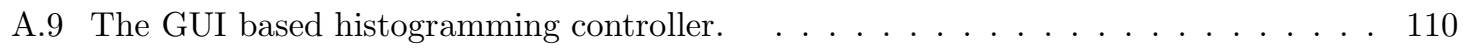

B.1 The distribution of NN input variables for the Medium muon definition. Muon variables. From left to right, muon $p_{\mathrm{T}}, p_{t}^{r e l}, \Delta R$ (muon, jet), $\chi^{2}$ and IP significance. The red histogram is for b-jets and the blue histogram is for uds-jets. . . . . . . . . . . 117

B.2 The distribution of NN input variables for the Medium muon definition. SVT variables. From left to right, $S V T_{S L}^{\text {mutrk }} D L S, S V T_{S L}^{\text {mutrk }} \chi_{\text {ndof }}^{2}, S V T_{S L}^{\text {mutrk }} N_{\text {tracks }}, S V T_{S L}^{\text {mutrk }} M a s s$, $S V T_{S L}^{m u t r k} N u m$, and CSIPcomb The red histogram is for b-jets and the blue histogram is for uds-jets. . . . . . . . . . . . . . . . . . 118

B.3 Comparison of the distributions for the $\mathrm{NN}$ input variables between data and $\mathrm{MC}$ sample on b-jets for Tight muon definition. Muon variables. From left to right, muon $p_{\mathrm{T}}, p_{t}^{r e l}, \Delta R$ (muon, jet), $\chi^{2}$ and IP significance. The red histogram is for b-jets from data sample and the blue histogram is for b-jets from the directbb MC sample. . . 119

B.4 Comparison of the distributions for the $\mathrm{NN}$ input variables between data and $\mathrm{MC}$ sample on b-jets for Tight muon definition. SVT variables. From left to right, $S V T_{S L}^{m u t r k} D L S, S V T_{S L}^{m u t r k} \chi_{n d o f}^{2}, S V T_{S L}^{m u t r k} N_{\text {tracks }}, S V T_{S L}^{m u t r k} M a s s, S V T_{S L}^{m u t r k} N u m$, and CSIPcomb. The red histogram is for b-jets from data sample and the blue histogram is for b-jets from the directbb MC sample. . . . . . . . . . . . . . . 120

B.5 Outputs for the NN training for each muon definition: Loose (top left), Medium (top right), Medium3 (bottom left) and Tight (bottom right). The red histogram is background (light-jets) from the QCD and $\gamma+$ jets combined MC samples, and the blue histogram is signal (b-jets) from the direct bb MC sample. . . . . . . . . . . . 121 
B.6 2D plot for Efficiency vs. Fake Rate measured in MC samples. The y-axis is the b-tagging efficiency (the SLTNN tagging efficiency) measured in the direct bb MC sample and the $\mathrm{x}$-axis is the fake rate determined in the $\mathrm{QCD}+\gamma+$ jets combined MC sample. The large points indicate the 3 operating points (Tight, Medium, and Loose from left to right) in this study for each muon definition. $\ldots \ldots \ldots \ldots$

B.7 2D plot for Efficiency vs. Fake Rate measured in MC samples. The y-axis is the c-tagging efficiency (the SLTNN tagging efficiency) measured in the direct cc MC sample and the $\mathrm{x}$-axis is the fake rate determined in the $\mathrm{QCD}+\gamma+$ jets combined MC sample. The large points indicate the 3 operating points (Tight, Medium, and Loose from left to right) in this study for each muon definition. . . . . . . . .

B.8 The muon-tagging efficiency for b-jets (Left) and c-jets (right), Loose muon definition. The red histogram denotes $t \bar{t}$ MC sample, the blue histogram denotes $Z \rightarrow b b(c c)$, the purple histogram denotes direct $\mathrm{bb}(\mathrm{cc})$ sample. From top to bottom, plots show the muon-tagging effciencies for CC, ICR, and EC regions, respectively. . . . . . . .

B.9 Measured systematic uncertainties for each $\eta$ region. From left to right, the plots show the results for Loose muon, Medium muon, Medium3 muon and Tight muon,

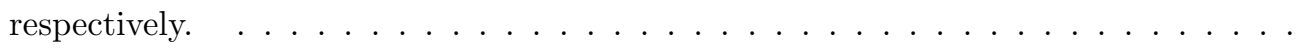

B.10 modJLIP distribution for each muon definition. Loose (top left), Medium (top right), Medium3 (bottom left), and Tight (bottom right). A modJLIP $<0.1$ cut is required

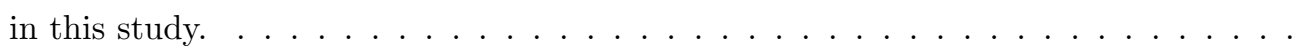

B.11 $\kappa_{b}$ for the Loose Muon and its Tight NN operating point. Top left (right) plot is the coefficient measured in jet $p_{\mathrm{T}}(\eta)$ bins. Bottom left (right) plot is the comparison between coefficients measured in $Z \rightarrow b b \rightarrow \mu$ and $t \bar{t}$ MC samples, respectively. The red line indicates $\varepsilon_{b}^{S L T N N}$, the pink line indicates $\varepsilon_{b}^{\operatorname{modJLIP}}$, the blue line indicates $\varepsilon_{b}^{S L T N N, \operatorname{modJLIP}}$. The black line indicates the coefficient estimated by fitting with a zeroth order polynomial function. . . . . . . . . . . . . . . . . 130 
B.12 $\kappa_{c l}$ for the Medium Muon and its Medium NN operating point. Top left (right) plot is the coefficient measured in jet $p_{\mathrm{T}}(\eta)$. Bottom left (right) plot is the comparison between coefficients measured in $Z \rightarrow c c \rightarrow \mu$ and directcc MC samples, respectively. The red line indicates $\varepsilon_{c l}^{S L T N N}$, the pink line indicates $\varepsilon_{c l}^{\text {modJLIP }}$, the blue line indicates $\varepsilon_{c l}^{S L T N N, \bmod J L I P}$. The black line indicates the coefficient estimated by fitting with a zeroth order polynomial function. . . . . . . . . . . . . . 131

B.13 $\beta$ for the Medium3 Muon and its Loose NN operating point. Top left (right) plot is the coefficient measured in jet $p_{\mathrm{T}}(\eta)$. Bottom left (right) plot is the comparison between coefficients measured in $Z \rightarrow b b \rightarrow \mu$ and $t \bar{t}$ MC samples, respectively. The red (blue) line indicates $\varepsilon^{S L T N N}$ meaured in muonic (b-enriched) sample. The black line indicates the coefficient estimated by fitting with a zeroth order polynomial function. 132

B.14 $\alpha$ for the Tight Muon and its Tight NN operating point. Top left (right) plot is the coefficient measured in jet $p_{\mathrm{T}}(\eta)$. Bottom left (right) plot is the comparison between coefficients measured in $Z \rightarrow c c \rightarrow \mu$ and directcc MC samples, respectively. The red (blue) line indicates $\varepsilon^{S L T N N}$ meaured in muonic (b-enriched) sample. The black line indicates the coefficient estimated by fitting with a zeroth order polynomial function. 133

B.15 b-tagging efficiency and scale factor for the Tight muon, Tight NN operating point. Left plots are the b-tagging efficiency of data (red) and MC sample (blue). Right plots are the scale factors for jet $p_{\mathrm{T}}$ (top) and $\eta$ (bottom). The dashed lines denote estimated statistical uncertainties. . . . . . . . . . . . . . . . . 136

B.16 $T R F_{b}$ (top) and $T R F_{c}$ (bottom) measured in the medium3 muon, medium NN operating point. Left plots are for the jet $p_{\mathrm{T}}$ distribution and right plots are for the jet $\eta$ distribution. The blue line is the MC b-tagging efficiency and the black line is the TRF. The dashed lines denotes estimated statistical uncertainties. $\quad \ldots \ldots \ldots$. . 137

B.17 The fake rate as fuction of jet $p_{\mathrm{T}}$ measured in the loose muon, tight NN operating point, CC region. $F_{h f}$ (top left), $F_{l l}$ (top right), $\varepsilon_{\text {data }}^{-}$(bottom left), and $\varepsilon_{\text {light }}$ (bottom right). . . . . . . . . . . . . . . . . . . . . . 140 
B.18 Electron $p_{\mathrm{T}}$ distributions for single tagged sample in the $e+$ jets channel. The plots show the data for different jet multiplicities: $=1$ jet (top left), $=2$ jets (top right), $=3$ jets (bottom left), and $\geq 4$ jets (bottom right). . . . . . . . . . . . 143

B.19 $E_{T}$ distributions for single tagged sample in the $e+$ jets channel. The plots show the data for different jet multiplicities: $=1$ jet (top left), $=2$ jets (top right), $=3$ jets (bottom left), and $\geq 4$ jets (bottom right). . . . . . . . . . . . . 143

B.20 Leading jet $p_{\mathrm{T}}$ distributions for single tagged sample in the $e+$ jets channel. The plots show the data for different jet multiplicities: $=1$ jet (top left), $=2$ jets (top right), $=3$ jets $($ bottom left), and $\geq 4$ jets (bottom right) . . . . . . . . . . . . . . 144

B.21 The $e \nu$ transverse mass distributions for single tagged sample in the $e+$ jets channel. The plots show the data for different jet multiplicities: $=1$ jet (top left),$=2$ jets (top right), $=3$ jets (bottom left), and $\geq 4$ jets (bottom right). . . . . . . . . . 144

B.22 Muon $p_{\mathrm{T}}$ distributions for single tagged sample in the $\mu+$ jets channel. The plots show the data for different jet multiplicities: $=1$ jet (top left), $=2$ jets (top right), $=3$ jets

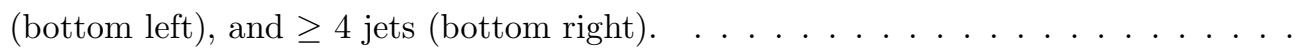

B.23 $E_{T}$ distributions for single tagged sample in the $\mu+$ jets channel. The plots show the data for different jet multiplicities: $=1$ jet (top left), $=2$ jets (top right), $=3$ jets

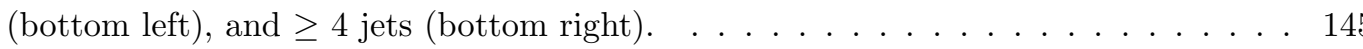

B.24 Leading jet $p_{\mathrm{T}}$ distributions for single tagged sample in the $\mu+$ jets channel. The plots show the data for different jet multiplicities: $=1$ jet (top left), $=2$ jets (top right), $=3$ jets (bottom left), and $\geq 4$ jets (bottom right). . . . . . . . . . . . 146

B.25 The $\mu \nu$ transverse mass distributions for single tagged sample in the $\mu+$ jets channel. The plots show the data for different jet multiplicities: $=1$ jet (top left), $=2$ jets (top

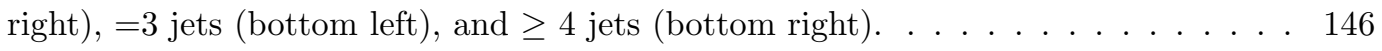

B.26 The signal and background composition predicted using MC samples and the number of events in the data sample as a function of jet multiplicity for the SLTNN tagging algorithm. The plot shows the result in the lepton+jets channel. . . . . . . . . . . . 149 
B.27 Summary of the various $t \bar{t}$ cross section measurements made by CDF and DØ. The shade areas denote the theoretical cross sections with its uncertainty. The red bars denote the statistical uncertainty and the blue bars denote the total uncertainty for the measurement. . . . . . . . . . . . . . . . . . . . . . 151

C.1 Centrality distributions for the preselected sample in the $e+$ jets channel. $\quad \ldots \ldots$. . 153

C.2 Sphericity distributions for the preselected sample in the $e+$ jets channel. . . . . . . 154

C.3 $\quad H_{T}$ distributions for the preselected sample in the $e+$ jets channel. . . . . . . . 154

C.4 $\Delta \phi$ (leading Jet, $\mathbb{E}_{T}$ ) distributions for the preselected sample in the $e+$ jets channel. . 155

C.5 $\Delta \phi\left(\right.$ electron, $\left.E_{T}\right)$ distributions for the preselected sample in the $e+$ jets channel. $\quad . \quad 155$

C.6 Electron $p_{\mathrm{T}}$ distributions for the exactly one tagged sample in the $e+$ jets channel. . 156

C.7 $E_{T}$ distributions for the exactly one tagged sample in the e+jets channel. . . . . . 157

C.8 Leading jet $p_{\mathrm{T}}$ distributions for the exactly one tagged sample in the $e+$ jets channel. 157

C.9 The $e \nu$ transverse mass distributions for the exactly one tagged sample in the $e+$ jets

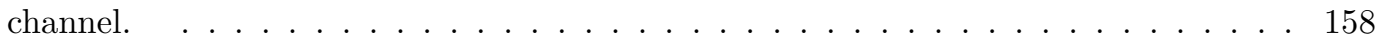

C.10 Centrality distributions for the exactly one tagged sample in the $e+$ jets channel. . . 158

C.11 Sphericity distributions for the exactly one tagged sample in the e+jets channel. . . 159

C.12 $H_{T}$ distributions for the exactly one tagged sample in the $e+$ jets channel. . . . . . 159

C.13 $\Delta \phi$ (leading Jet, $\left.\mathbb{E}_{T}\right)$ distributions for the exactly one tagged sample in the $e+$ jets

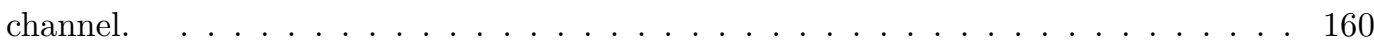

C.14 $\Delta \phi\left(\right.$ electron, $\left.\mathbb{E}_{T}\right)$ distributions for the exactly one tagged sample in the $e+$ jets channel. 160

C.15 Electron $p_{\mathrm{T}}$ distributions for the two or more tagged sample in the $e+$ jets channel. $\quad 161$

C.16 $E_{T}$ distributions for the two or more tagged sample in the $e+$ jets channel. $\quad \ldots \ldots$.

C.17 Leading jet $p_{\mathrm{T}}$ distributions for the two or more tagged sample in the $e+$ jets channel. 162

C.18 The $e \nu$ transverse mass distributions for the two or more tagged sample in the $e+$ jets channel. . . . . . . . . . . . . . . . . . . . . 163

C.19 Centrality distributions for the two or more tagged sample in the e+jets channel. $\quad$. 163

C.20 Sphericity distributions for the two or more tagged sample in the $e+$ jets channel. $\quad$. 164 
C.21 $H_{T}$ distributions for the two or more tagged sample in the $e+$ jets channel. $\quad \ldots . . . \quad 164$

C.22 $\Delta \phi$ (leading Jet, $\left.\mathbb{E}_{T}\right)$ distributions for the two or more tagged sample in the $e+$ jets

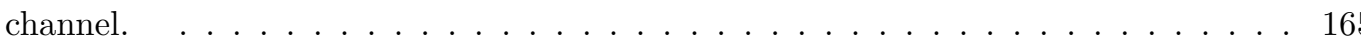

C.23 $\Delta \phi\left(\right.$ electron, $\left.E_{T}\right)$ distributions for the two or more tagged sample in the $e+$ jets chan-

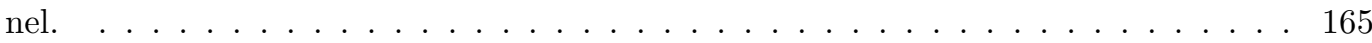

C.24 Centrality distributions for the preselected sample in the $\mu+$ jets channel. $\quad \ldots \ldots$. 166

C.25 Sphericity distributions for the preselected sample in the $\mu+$ jets channel. $\ldots \ldots \ldots$. 167

C.26 $H_{T}$ distributions for the preselected sample in the $\mu+$ jets channel. . . . . . . . . 167

C.27 $\Delta \phi$ (leading Jet, $\left.\mathbb{E}_{T}\right)$ distributions for the preselected sample in the $\mu+$ jets channel. $\quad 168$

C.28 $\Delta \phi\left(\right.$ muon,$\left.E_{T}\right)$ distributions for the preselected sample in the $\mu+$ jets channel. $\ldots . .168$

C.29 Muon $p_{\mathrm{T}}$ distributions for the exactly one tagged sample in the $\mu+$ jets channel. $\quad$. 169

C.30 $E_{T}$ distributions for the exactly one tagged sample in the $\mu+$ jets channel. $\ldots \ldots \ldots 170$

C.31 Leading jet $p_{\mathrm{T}}$ distributions for the exactly one tagged sample in the $\mu+$ jets channel. 170

C.32 The $\mu \nu$ transverse mass distributions for the exactly one tagged sample in the $\mu+$ jets

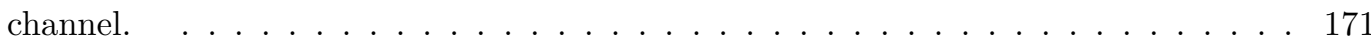

C.33 Centrality distributions for the exactly one tagged sample in the $\mu+$ jets channel. . . 171

C.34 Sphericity distributions for the exactly one tagged sample in the $\mu+$ jets channel. $\quad$. 172

C.35 $H_{T}$ distributions for the exactly one tagged sample in the $\mu$ +jets channel. $\quad \ldots \ldots$. 172

C.36 $\Delta \phi$ (leading Jet, $\left.E_{T}\right)$ distributions for the exactly one tagged sample in the $\mu+$ jets channel. . . . . . . . . . . . . . . . . . . . . . . . 173

C.37 $\Delta \phi\left(\right.$ muon,$\left.E_{T}\right)$ distributions for the exactly one tagged sample in the $\mu+$ jets channel. 173

C.38 Muon $p_{\mathrm{T}}$ distributions for the two or more tagged sample in the $\mu+$ jets channel. . . 174

C.39 $E_{T}$ distributions for the two or more tagged sample in the $\mu+$ jets channel. $\quad \ldots \ldots$. 175

C.40 Leading jet $p_{\mathrm{T}}$ distributions for the two or more tagged sample in the $\mu+$ jets channel. 175

C.41 The $\mu \nu$ transverse mass distributions for the two or more tagged sample in the $\mu+$ jets channel. . . . . . . . . . . . . . . . . . . . . . . . 176

C.42 Centrality distributions for the two or more tagged sample in the $\mu+$ jets channel. $\quad 176$ 
C.43 Sphericity distributions for the two or more tagged sample in the $\mu+$ jets channel. $\quad$ • 177

C.44 $H_{T}$ distributions for the two or more tagged sample in the $\mu$ +jets channel. $\quad$. . . . 177

C.45 $\Delta \phi\left(\right.$ leading Jet, $\left.\mathbb{E}_{T}\right)$ distributions for the two or more tagged sample in the $\mu+$ jets channel. ............................. . . . . . . . . . . . . . . . . . .

C.46 $\Delta \phi\left(\right.$ muon, $\left.\mathbb{E}_{T}\right)$ distributions for the two or more tagged sample in the $\mu+$ jets channel. 178 


\section{Chapter 1}

\section{Introduction}

The top quark, known as the heaviest fundamental particle, was discovered in 1995 by both the CDF and DØ collaborations $[1,2]$. However, studies of the top quark are still at the beginning stage and its characteristics are still under a veil of mystery. Moreover, studying properties of the top quark is interesting because it gives insights into Higgs search, new physics beyond the Standard Model (SM), electroweak symmetry breaking, and perturbative Quantum Chromo Dynamics (QCD). Therefore, top quark physics is one of the most interesting topics in High Energy Physics (HEP) today and the Tevatron accelerator at Fermilab is currently the only place where the top quark can be created and studied until the Large Hadron Collider (LHC) at CERN starts taking data. In this chapter, the SM, which describes fundamental particles and their interactions well, is generally discussed and the production of top quark pair events at the Tevatron is also described.

\section{$1.1 \quad$ Standard Model}

What does our world consist of? This question always stimulates people's intellectual curiosity. From ancient times, scientists have devoted their efforts finding the smallest units of matter and how these units interact with each other. We believe at present that there are two types of the smallest units of matter, leptons and quarks, and four different fundamental interactions (forces), gravity, electromagnetism, weak interaction, and strong interaction. The theories that describe these 
units and interactions are collectively called "the Standard Model".

The quark has 6 flavors (up, down, strange, charm, bottom, and top) at present and they fall into doublets called "generation"

$$
\left(\begin{array}{l}
u \\
d
\end{array}\right)\left(\begin{array}{l}
c \\
s
\end{array}\right)\left(\begin{array}{l}
t \\
b
\end{array}\right)
$$

where $\mathrm{u}$ is up, $\mathrm{d}$ is down, $\mathrm{s}$ is strange, $\mathrm{c}$ is charm, $\mathrm{b}$ is bottom and $\mathrm{t}$ is top. The top row in the doublets has $2 / 3$ electric charge and the bottom row has $-1 / 3$. The quark also carries a charge of QCD, called color charge. There are three colors: red, blue, and green.

The lepton also has 6 flavors with three generations of doublet

$$
\left(\begin{array}{c}
\nu_{e} \\
e
\end{array}\right)\left(\begin{array}{c}
\nu_{\mu} \\
\mu
\end{array}\right)\left(\begin{array}{c}
\nu_{\tau} \\
\tau
\end{array}\right)
$$

where $e$ is electron, $\mu$ is muon and $\tau$ is tau. $\nu$ is the neutrino and the subscript is the generation associated with one of $e, \mu$ and $\tau$. The summary of charateristics for quarks and leptons is shown in Table 1.1.

\begin{tabular}{|c|c|c|c|c|c|c|}
\hline \hline Type & Name & Mass $[3]$ & Spin & Charge $(Q)$ & $N_{\text {baryon }}(B)$ & $N_{\text {lepton }}(L)$ \\
\hline \multirow{5}{*}{ Quarks } & $\mathrm{u}$ & $1.5 \sim 3.0 \mathrm{MeV}$ & $\frac{1}{2}$ & $+\frac{2}{3}$ & $\frac{1}{3}$ & 0 \\
& $\mathrm{~d}$ & $3 \sim 7 \mathrm{MeV}$ & $\frac{1}{2}$ & $-\frac{1}{3}$ & $\frac{1}{3}$ & 0 \\
& $\mathrm{~s}$ & $95 \pm 25 \mathrm{MeV}$ & $\frac{1}{2}$ & $-\frac{1}{3}$ & $\frac{1}{3}$ & 0 \\
& $\mathrm{c}$ & $1.25 \pm 0.09 \mathrm{GeV}$ & $\frac{1}{2}$ & $+\frac{2}{3}$ & $\frac{1}{3}$ & 0 \\
& $\mathrm{~b}$ & $4.20 \pm 0.07 \mathrm{GeV}$ & $\frac{1}{2}$ & $-\frac{1}{3}$ & $\frac{1}{3}$ & 0 \\
& $\mathrm{t}$ & $174.2 \pm 3.3 \mathrm{GeV}$ & $\frac{1}{2}$ & $+\frac{2}{3}$ & $\frac{1}{3}$ & 0 \\
\hline \multirow{6}{*}{ Leptons } & $\mathrm{e}$ & $0.51 \pm 0.00 \mathrm{MeV}$ & $\frac{1}{2}$ & -1 & 0 & 1 \\
& $\nu_{e}$ & $<2 \mathrm{eV}$ & $\frac{1}{2}$ & 0 & 0 & 1 \\
& $\mu$ & $105.66 \pm 0.00 \mathrm{MeV}$ & $\frac{1}{2}$ & -1 & 0 & 1 \\
& $\nu_{\mu}$ & $<0.19 \mathrm{MeV}$ & $\frac{1}{2}$ & 0 & 0 & 1 \\
& $\tau$ & $1776.99{ }_{-0.26}^{+0.29} \mathrm{MeV}$ & $\frac{1}{2}$ & -1 & 0 & 1 \\
& $\nu_{\tau}$ & $<18.2 \mathrm{MeV}$ & $\frac{1}{2}$ & 0 & 0 & 1 \\
\hline \hline
\end{tabular}

Table 1.1: The summary of characteristics for quarks and leptons.

In addition to the quarks and leptons, there are basic elementary particles which transmit the 
four fundamental forces, called "gauge bosons". The graviton mediates gravity, the photon $(\gamma)$ mediates the electromagnetism, the $W^{ \pm}$and $Z^{0}$ mediate the weak interaction which is responsible for neutrino $(\nu)$ interactions and nuclear beta decay, and the gluons mediate the strong interaction which binds the quarks in the proton. These interactions and the behavior of elementary particles can be calculated by quantum field theory. Table 1.2 shows a summary of the four forces and their gauge bosons.

\begin{tabular}{|c|c|c|c|}
\hline \hline Force & act on & gauge bosons & characteristics \\
\hline Gravity & all particles & graviton & massless, spin -2 \\
Electromagnetism & all charged particles & photon & massless, spin -1 \\
Weak Interaction & quarks, leptons, electroweak gauge bosons & $W^{ \pm}, Z^{0}$ & heavy, spin -1 \\
Strong Interaction & quarks, gluons (color charged) & eight gluons & massless, spin -1 \\
\hline \hline
\end{tabular}

Table 1.2: Summary of the four fundamental forces and their gauge bosons [4].

The electromagnetic and weak interactions are unified in the SM, and are called the "electroweak interaction" with $S U(2)_{L} \times U(1)_{Y}$ symmetry. Four vector fields are introduced to describe the electroweak interaction: two massive charged bosons $W^{ \pm}$and two neutral fields $Z^{0}$ and $\gamma$. In the quantum field theory, the gauge bosons are massless, but in the unified electroweak interaction, $W^{ \pm}$and $Z^{0}$ have heavy masses. Their masses can be explained by spontaneous symmetry breaking. Spontaneous breaking of the local $U(1)$ and $S U(2)$ gauge symmetries makes the $W^{ \pm}$and $Z^{0}$ massive and the photon remains massless. This is called Higgs mechanism. The scalar field resulting from the Higgs mechanism is called the "Higgs boson" and the search for it is one of the major challenges in elementary particle physics.

The strong interaction can be described by the QCD theory, which has a $S U(3)$ gauge symmetry for phase transformations on the quark color fields. Each quark has three color flavors and they form a triplet in the $S U(3)$ group. There exist eight gluons to mediate the strong interaction, and they form an octet in the adjoint representation by the product $3 \otimes 3=1 \oplus 8$. The effect of strong interactions at large $\mathrm{Q}^{2}$ can be calculated by perturbative QCD. The top quark pair events are produced by this strong interaction at the Tevatron. The theoretical aspects of the production will 
be described in the next section.

\subsection{Top Quark Pair Production and Decay}

The top quark can be produced at the Tevatron (a proton-antiproton collider) in two different ways: pair production of the top quarks (namely, $t \bar{t}$ events) and Drell-Yan production of single top quark (called single top). The $t \bar{t}$ events can be produced by strong interaction through the processes $q \bar{q}$ annihilation and gluon fusion, shown in Figure 1.1. At the Tevatron, the contributions of these two processes are $85 \%$ and $15 \%$ at $\sqrt{s}=1.96 \mathrm{TeV}$, respectively. In this thesis, QCD pair production of the top quark is examined.

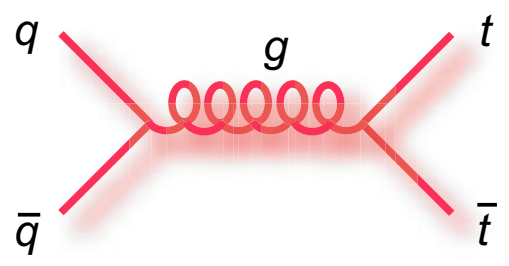

(a) $q \bar{q}$ annihilation process
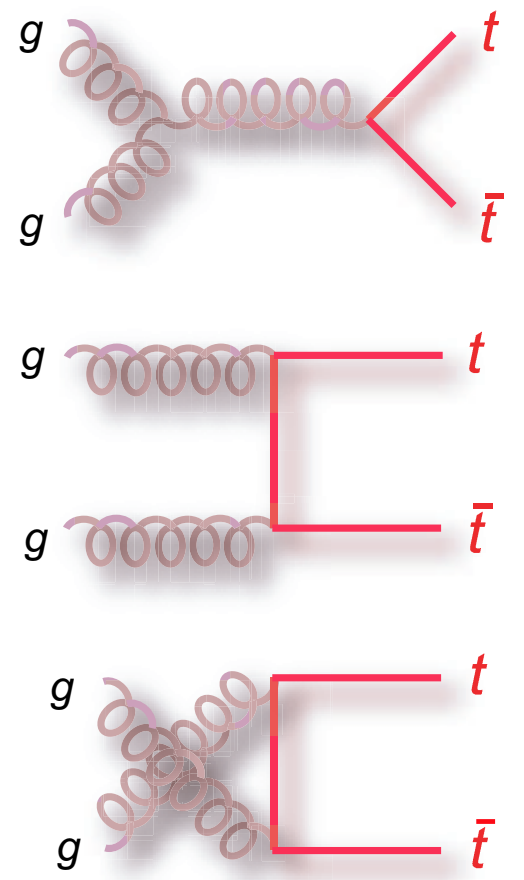

(b) gluon fusion process

Figure 1.1: The lowest order processes for $t \bar{t}$ pair production in QCD [5]. 


\subsubsection{Total Cross Section for the Top Quark Pair Production}

The total cross section for $t \bar{t}$ pair events, $p \bar{p} \rightarrow t \bar{t}$, at a center of mass $\sqrt{s}$ can be calculated by the following formula $[6]$

$$
\sigma(s)=\sum_{i, j=q, \bar{q}, g} \int d x_{i} d x_{j} \hat{\sigma}_{i, j}\left(x_{i} x_{j} s, m_{t}^{2}, \mu^{2}\right) F_{i}^{p}\left(x_{i}, \mu\right) F_{j}^{\bar{p}}\left(x_{j}, \mu\right)
$$

where $i$ and $j$ indicate light quarks and gluons for $t \bar{t}$ pair event production, for example $q \bar{q} \rightarrow t \bar{t}$ and $g+g \rightarrow t \bar{t}$ in the Leading Order (LO) production (Figure 1.1). The $F_{i}^{p}$ and $F_{j}^{\bar{p}}$ are the parton distribution functions (PDF), the probability densities of finding a parton with a given momentum fraction for $p$ and $\bar{p}$, evaluated at a scale $\mu . \mu$ is the renomalization and factorization scale. $x_{i}$ and $x_{j}$ denote the momentum fractions of $i$ and $j$ partons respectively.

The symbol $\hat{\sigma}$ is the subprocess (parton-parton) cross section for $t \bar{t}$ events $\left(\mu \approx m_{t}\right)$. It is calculable as a perturbation series in terms of $\alpha_{S}\left(\mu^{2}\right)$, a coupling constant of the strong interaction. The formula for $\hat{\sigma}$ is

$$
\hat{\sigma}_{i, j}\left(s, m_{t}^{2}, \mu^{2}\right)=\frac{\alpha_{S}^{2}\left(\mu^{2}\right)}{m_{t}^{2}} f_{i j}\left(\rho, \frac{\mu^{2}}{m_{t}^{2}}\right)
$$

where $\rho=4 m_{t}^{2} / s$ and $s$ is the square of the partonic center of mass energy. In this formula, the dimensionless function $f_{i j}$ can be expanded as following

$$
f_{i j}\left(\rho, \frac{\mu^{2}}{m_{t}^{2}}\right)=f_{i j}^{(0)}(\rho)+g^{2}\left(\mu^{2}\right)\left[f_{i j}^{(1)}(\rho)+\bar{f}_{i j}^{(1)}(\rho) \ln \left(\frac{\mu^{2}}{m_{t}^{2}}\right)\right]+O\left(g^{4}\right)
$$

where the coupling constant $\alpha_{S}=g^{2} / 4 \pi$. Therefore, the functions $f_{i j}^{(0)}$ are the LO contributions $\left(O\left(\alpha_{S}^{2}\right)\right)$, and the $f_{i j}^{(1)}$ and $\bar{f}_{i j}^{(1)}$ are the Next-to-Leading-Order (NLO) contributions $\left(O\left(\alpha_{S}^{3}\right)\right)$. According to Reference [6], the functions $f_{i j}^{(0)}$ are

$$
\begin{aligned}
f_{q \bar{q}}^{(0)}(\rho) & =\frac{\pi \beta \rho}{27}[2+\rho] \\
f_{g g}^{(0)}(\rho) & =\frac{\pi \beta \rho}{192}\left[\frac{1}{\beta}\left(\rho^{2}+16 \rho+16\right) \ln \left(\frac{1+\beta}{1-\beta}\right)-28-31 \rho\right]
\end{aligned}
$$




$$
f_{g q}^{(0)}(\rho)=f_{g \bar{q}}^{(0)}(\rho)=0
$$

where $\beta=\sqrt{1-\rho} . \quad f_{i j}^{(1)}$ and $\bar{f}_{i j}^{(1)}$ can be numerically calculated and represented by a physically motivated fit function. The results of functions are presented in Reference [6]. Using these functions, the total cross section can be evaluated theoretically in Equation 1.1. The NLO results determined by this calculation are shown in Figure 1.2 and Table 1.3.

A few years ago, the Next-to-Next-to-Leading-Order (NNLO) correction at $\sqrt{s}=1.96 \mathrm{TeV}$ was calculated in References [7, 8]. The results in Reference [7] are shown in Figure 1.2 and Table 1.3. The best estimation in the reference is $\sigma_{t \bar{t}}=6.77 \pm 0.42 \mathrm{pb}$ at $\mu=m_{t}=175 \mathrm{GeV}$. The uncertainty is from the choice of the PDF. The results in Reference [8] are shown in Table 1.4.

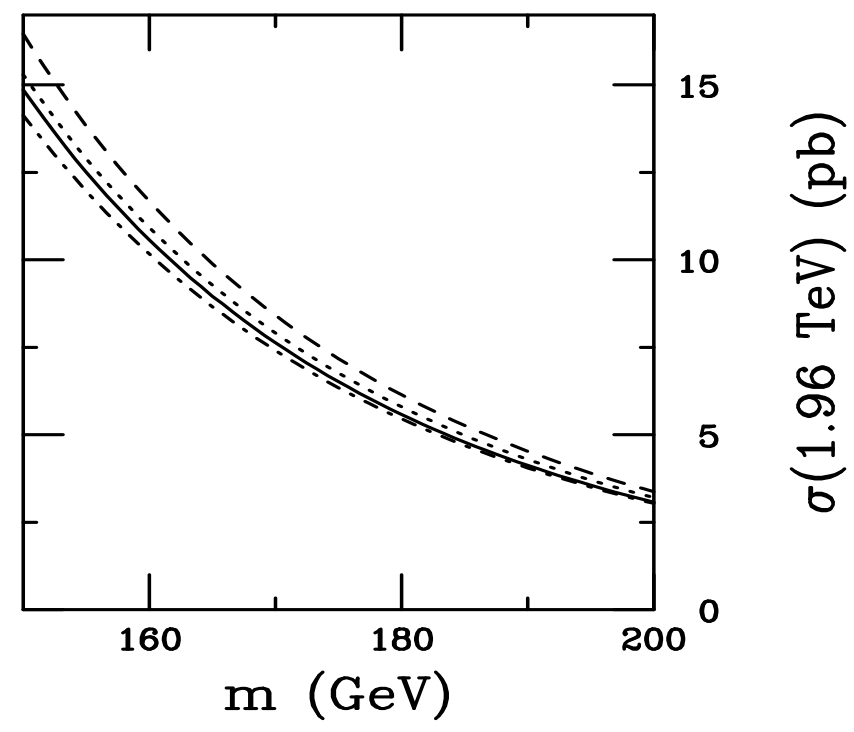

Figure 1.2: The $t \bar{t}$ cross section in $p \bar{p}$ collision at $\sqrt{S}=1.96 \mathrm{TeV}$ as a function of $m_{t}$ for $\mu=m_{t}$. The NLO (solid) and NNLO (dotted) with uncertainties (dashed and dot-dashed) [7].

\subsubsection{Top Quark Decay}

The world average of the top quark mass measurement is $172.6 \pm 0.8$ (stat) \pm 1.1 (sys) GeV at present [11]. The top quark has the heaviest mass of the discovered fundamental elementary particles and its lifetime is too short for it to hadronize due to its heavy mass. 


\begin{tabular}{|c|c|c|c|c|c|c|}
\hline \hline \multicolumn{9}{|c|}{$\sigma_{t \bar{t}}(\mathrm{pb})$} \\
\hline & \multicolumn{3}{|c|}{ MRST2002 NNO } & \multicolumn{3}{c|}{ CTEQ6M } \\
\hline Order & $\mu=m_{t} / 2$ & $\mu=m_{t}$ & $\mu=2 m_{t}$ & $\mu=m_{t} / 2$ & $\mu=m_{t}$ & $\mu=2 m_{t}$ \\
\hline NLO & 6.79 & 6.52 & 5.83 & 6.79 & 6.54 & 5.85 \\
NNLO 1PI & 7.00 & 7.17 & 6.99 & 7.01 & 7.21 & 7.04 \\
NNLO PIM & 6.14 & 6.35 & 6.28 & 6.08 & 6.33 & 6.29 \\
\hline \hline
\end{tabular}

Table 1.3: The $t \bar{t}$ pair production cross section in $p \bar{p}$ collision at Tevatron $(\sqrt{s}=$ $1.96 \mathrm{TeV}$ ) for $m_{t}=175 \mathrm{GeV}$ [7]. MRST2002 [9] and CTEQ6M [10] are parton distribution functions. 1PI denotes single-particle-inclusive kinematics and PIM denotes pair-invariant-mass kinematics.

\begin{tabular}{|c|c|c|c|c|}
\hline \hline PDF & $m_{t}$ & $\sigma_{\min }(\mathrm{pb})$ & $\sigma_{r e f}(\mathrm{pb})$ & $\sigma_{\max }(\mathrm{pb})$ \\
\hline & 170 & 6.79 & 7.83 & 8.54 \\
CTEQ6M & 175 & 5.82 & 6.70 & 7.30 \\
& 180 & 5.00 & 5.75 & 6.25 \\
\hline & 170 & 7.11 & 7.90 & 8.31 \\
MRST & 175 & 6.08 & 6.76 & 7.10 \\
& 180 & 5.21 & 5.79 & 6.08 \\
\hline \hline
\end{tabular}

Table 1.4: The $t \bar{t}$ cross section predictions for the CTEQ6M and MRST parton distribution functions at $\sqrt{s}=1.96 \mathrm{TeV}[8]$.

The top quark decays into a $\mathrm{W}$ boson and a $\mathrm{b}$ quark before hadronization, $t \rightarrow W b$, with almost $100 \%$ branching fraction in the SM. The top quark can also decay to Ws and Wd by the Cabibbo-Kobayashi-Maskawa (CKM) mixing. However, the branching fraction is very small and it is not considered in this analysis. After decaying to the $\mathrm{W}$ boson and $\mathrm{b}$ quark, the $\mathrm{b}$ quark is hadronized. The $\mathrm{W}$ boson has two decay modes, leptonic decay and hadronic decay. Table 1.5 shows the branching fractions of the $\mathrm{W}$ boson decay modes [3].

\begin{tabular}{|c|c|c|}
\hline \hline \multicolumn{2}{|c|}{ Decay Mode $\left(W^{+}\right)$} & Branching Fraction \\
\hline \multirow{3}{*}{ Leptonic Decay } & $e^{+} \nu_{e}$ & $10.75 \pm 0.13 \%$ \\
& $\mu^{+} \nu_{\mu}$ & $10.57 \pm 0.15 \%$ \\
& $\tau^{+} \nu_{\tau}$ & $11.25 \pm 0.20 \%$ \\
\hline \multirow{2}{*}{ Hadronic Decay } & $u \bar{d}$ & $67.60 \pm 0.27 \%$ \\
& $c \bar{s}$ & \\
\hline \hline
\end{tabular}

Table 1.5: The branching fractions of the $\mathrm{W}$ boson decay mode [3]. 
Depending on the $\mathrm{W}$ boson decay mode, the analysis of $t \bar{t}$ pair events can be separated into three channels: dilepton channel, lepton+jets channel, and all-hadronic channel. The dilepton channel has both the $W^{+}$and $W^{-}$from $t \bar{t}$ decay leptonically, the lepton+jets channel has one of the W boson decays leptonically and the other W boson decays hadronically, and the all-hadronic channel has both the $\mathrm{W}$ bosons decay hadronically. The branching fraction for each channel is determined by the branching fractions of the $\mathrm{W}$ boson shown in Table 1.5. Figure 1.3 shows the decay channels for $t \bar{t}$ pair events and their branching fractions.

\section{Top Pair Decay Channels}

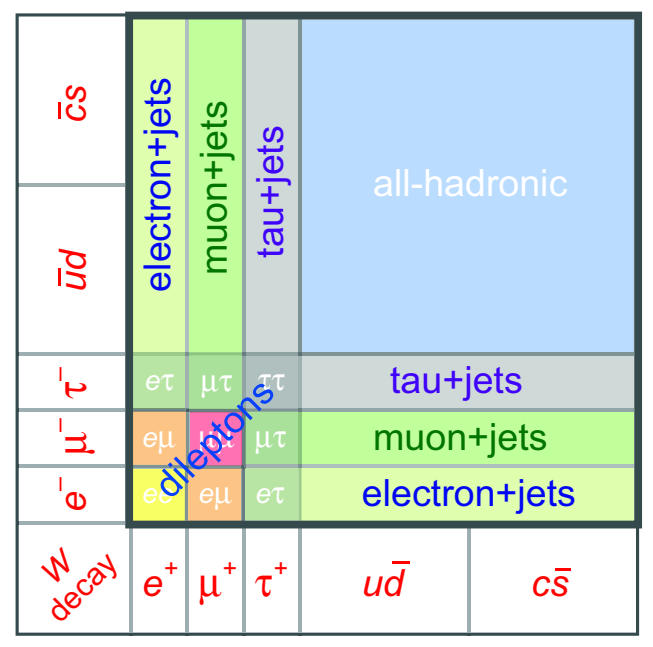

(a) The decay channels for $t \bar{t}$ pair events.

\section{Top Pair Branching Fractions}

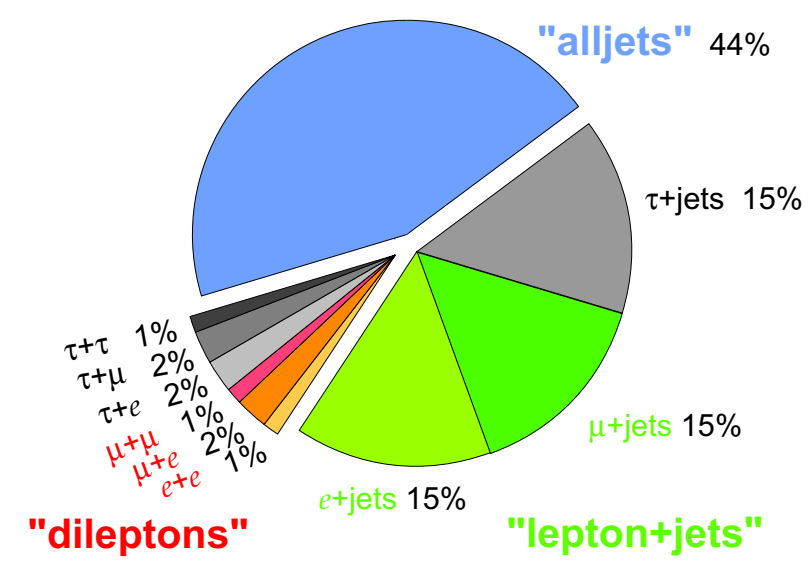

(b) The branching fractions for the decay channels of $t \bar{t}$ pair events.

Figure 1.3: Decay channels for $t \bar{t}$ pair events and their branching fractions [5]. 
In this thesis, only electron+jets and muon+jets channels, including the contributions from the $\tau+$ jets channel $(\tau \rightarrow e$ or $\tau \rightarrow \mu)$, are discussed. The $t \bar{t}$ pair events in the lepton + jets channel at DØ have one lepton, missing transverse energy $\left(E_{T}\right)$ originating from the neutrino, two jets from a $\mathrm{W}$ boson decaying hadronically, and two b-quark jets from $t \bar{t}$ decays in the final state. The lepton and $E_{T}$ from leptonic $\mathrm{W}$ boson decay have large transverse momentum because of the heavy mass of the $\mathrm{W}$ boson (about $80.4 \mathrm{GeV}$ ). Figure 1.4 shows the Feynman Diagram for $t \bar{t}$ events in the lepton+jets channel.

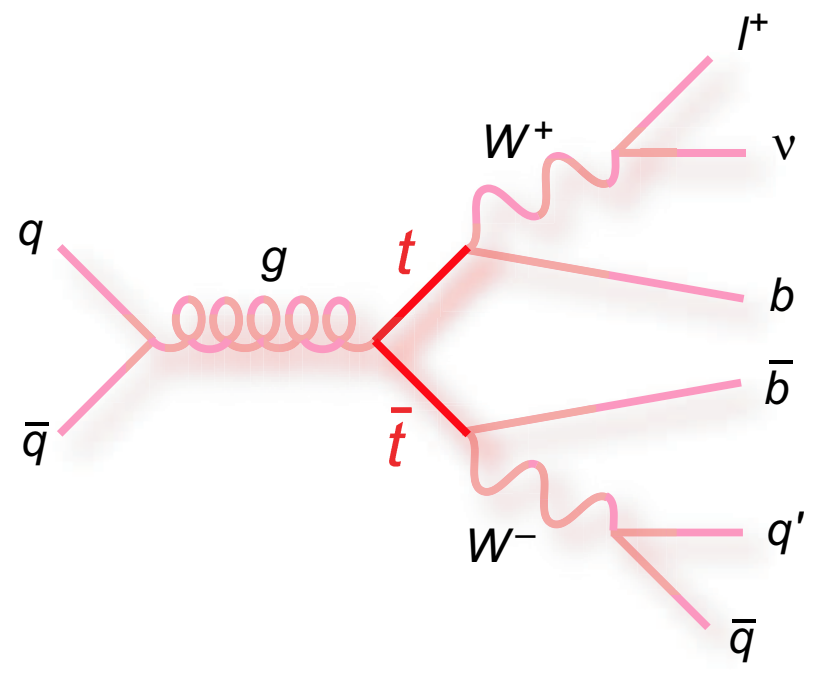

Figure 1.4: The event signature of $t \bar{t}$ pair production in the lepton+jets channel [5]. 


\section{Chapter 2}

\section{Tevatron and DØ Detector}

The Fermi National Accelerator Laboratory (Fermilab) [12] plays a leading role in the field of experimental particle physics of the world. At the Fermilab Tevatron collider, Run I (1992-1996) was successfully completed with about $130 p b^{-1}$ of delivered luminosity, resulting in the discovery of the top quark. Now in Run II (2002-) the accelerators and detectors are upgraded and data taking is under way.

\subsection{Tevatron}

The Fermilab accelerator [13] consists of a chain of accelerators: the Cockcroft-Walton injector, the LINAC (LINear ACcelerator), the Booster, the Main Injector, and the Tevatron as shown in Figure 2.1. In the Tevatron, protons and antiprotons are counter-rotating with $1.96 \mathrm{TeV}$ center of mass energy, making the Tevatron as the highest energy collider in the world until the Large Hadron Collider (LHC) at CERN starts to run. Futhermore, the Tevatron is currently the only collider with sufficient center of mass energy to produce top quark pair events.

Protons are accelerated to $400 \mathrm{MeV}$ in the Cockroft-Walton and the LINAC. The CockroftWalton is a $750 \mathrm{kV}$ DC voltage source in which $\mathrm{H}^{-}$ions are pre-accelerated to $750 \mathrm{KeV}$. The $\mathrm{H}^{-}$ ions are further accelerated in the LINAC, which is a $130 \mathrm{~m}$ long set of drift tubes divided by vacuum gaps. In the LINAC, an electric field is produced by radio frequency $(\mathrm{RF})$ power and it is polarized 


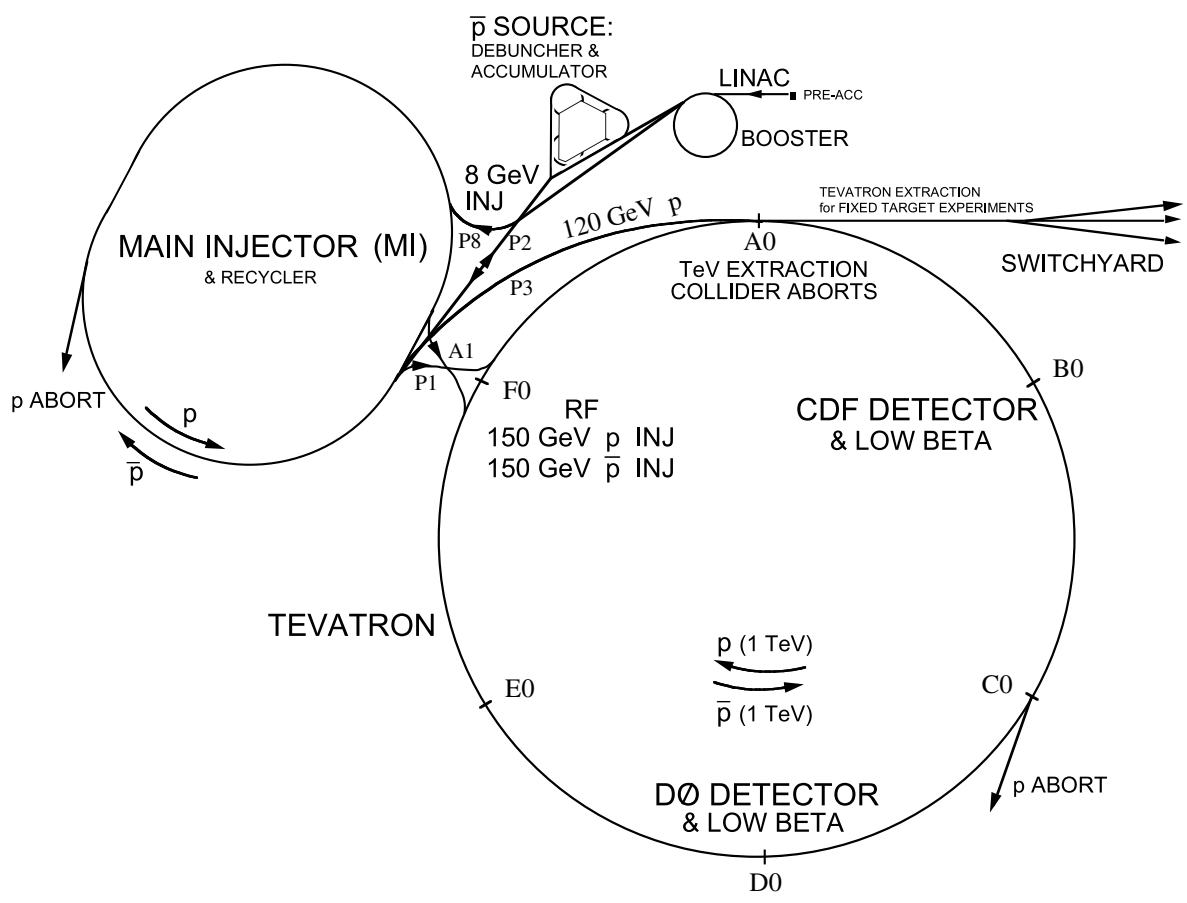

Figure 2.1: Fermilab accelerator complex.

in the same direction in all the gaps. The $\mathrm{H}^{-}$ions increase their speed in the gaps and keep the speed inside the drift tubes when the electric field reverses. The drift tubes become longer and longer as the velocity of $\mathrm{H}^{-}$increases as shown in Figure 2.2. Instead of a continuous ion beam, the ions are "bunched" together in the LINAC because of the alternating electric field. The bunches of ions are spaced $5 \mathrm{~ns}$ apart and the typical bunch contains 1.5 billion particles. After acceleration to $400 \mathrm{MeV}$, ions pass through a carbon foil to make bare protons. The bare protons are injected into the Booster, which is a $475 \mathrm{~m}$ circumference synchrotron. In the Booster, protons are bent into a circular orbit by dipole magnets and accelerated to $8 \mathrm{GeV}$ by RF cavities [14].

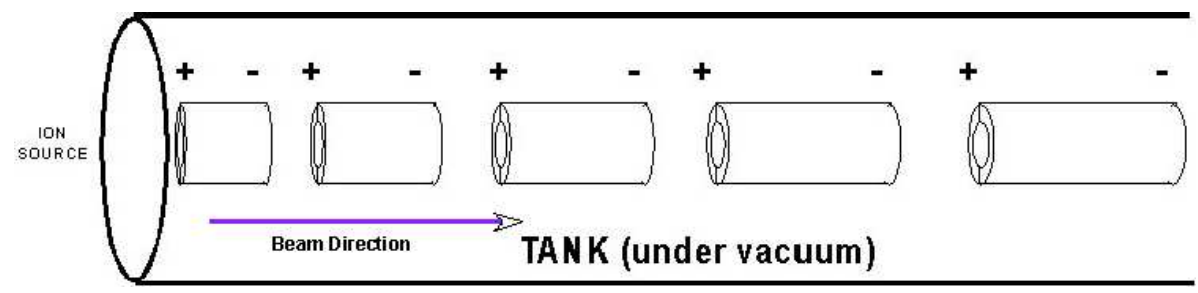

Figure 2.2: Diagram of the LINAC which shows how $\mathrm{H}^{-}$ions are accelerated [14].

After the Booster, protons are injected into the Main Injector. The Main Injector is a 2 mile 
circumference synchrotron that accelerates protons to $150 \mathrm{GeV}$. Another important role of the Main Injector is to produce antiprotons (Figure 2.3). Beams of $120 \mathrm{GeV}$ protons are collided into a nickel target every 1.5 second, and many secondary particles are created by these collisons. Particles other than antiprotons are filterd away by sending the beam through a pulsed magnet which acts as a charge-mass spectrometer. After that, the antiprotons move to the Debuncher and the Accumulater sequentially, which are $8 \mathrm{GeV}$ syncrotrons. In the Debuncher, higher energy antiprotons travel on the outside of the ring and lower energy ones on the inside of the ring. Whenever they pass through the RF cavity, the former is decelerated and the latter is accelerated. As this process happens over and over, eventually the energy spread of antiprotons will be reduced and they are debunched. Then, the antiprotons are sent to the Accumulator. Stochastic cooling technique is used to both the Debuncher and the Accumulator for cooling to reduce the beam emittance [15]. After accumulating a large number of antiprotons (over hours or days), they are sent back to the Main Injector and accelerated to $150 \mathrm{GeV}$. The Recycler is a fixed-energy storage ring for the antiprotons, and it is a leading factor in the increased luminosity of the Tevatron in Run II [16].

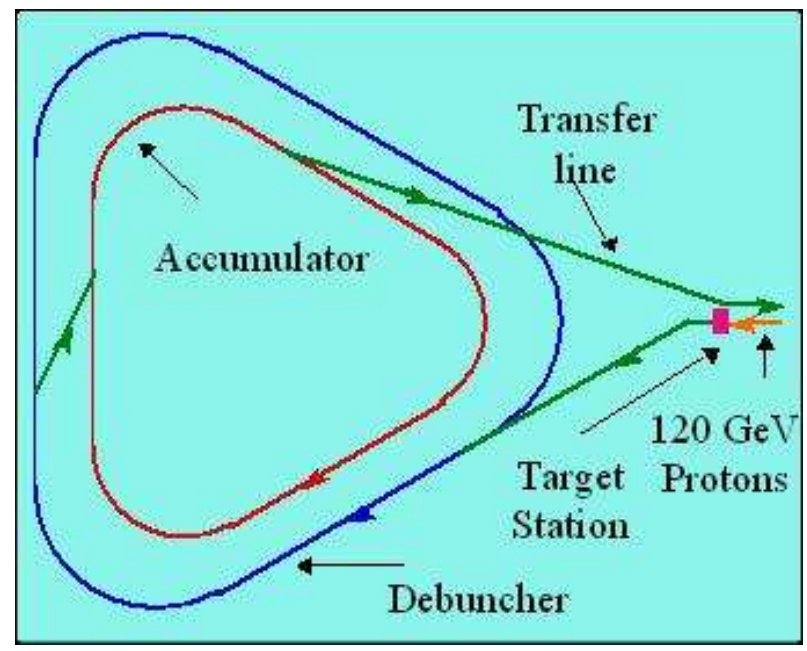

Figure 2.3: The diagram of the system used to accumulate and cool the antiprotons [15].

Both protons and antiprotons are injected into the Tevatron and accelerated to $980 \mathrm{GeV}$ individually. The Tevatron is the world's first superconducting syncrotron, and utilizes 1000 superconduncting magnets operating at $4.3 \mathrm{~K}$ [13]. The superconducting magnets produce a magnetic field of 4.2 Tesla, and the circumference of the Tevatron is 4 miles. Two circulating beams collide at 396 
ns time interval at two interaction points where the CDF and D $\varnothing$ detectors are located.

The Run II project began in 2001. It is split into two subsection: Run IIa and Run IIb. Run IIa was completed in April 2006 with an integrated luminosity of about $1.0 \mathrm{fb}^{-1}$, and Run IIb started in June 2006. Run IIb is planed to take data until 2009 with $8 \mathrm{fb}^{-1}$ expected to be recorded. Figure 2.4 shows (a) integrated luminosity and (b) the peak instantaneous luminosity at the Tevatron during Run II.

Collider Run II Integrated Luminosity

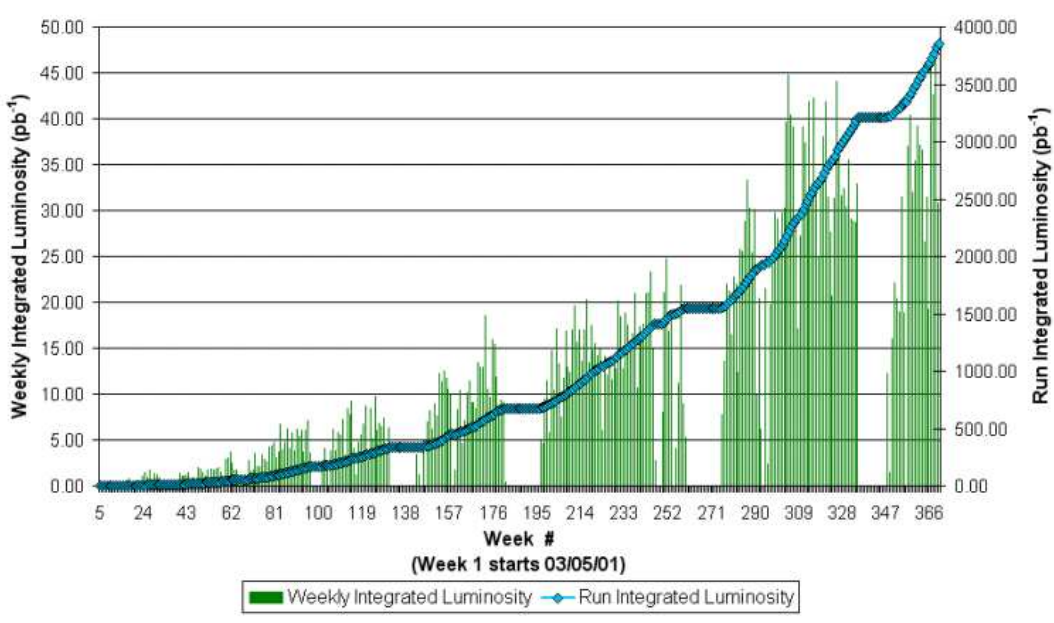

(a) Integrated Luminosity

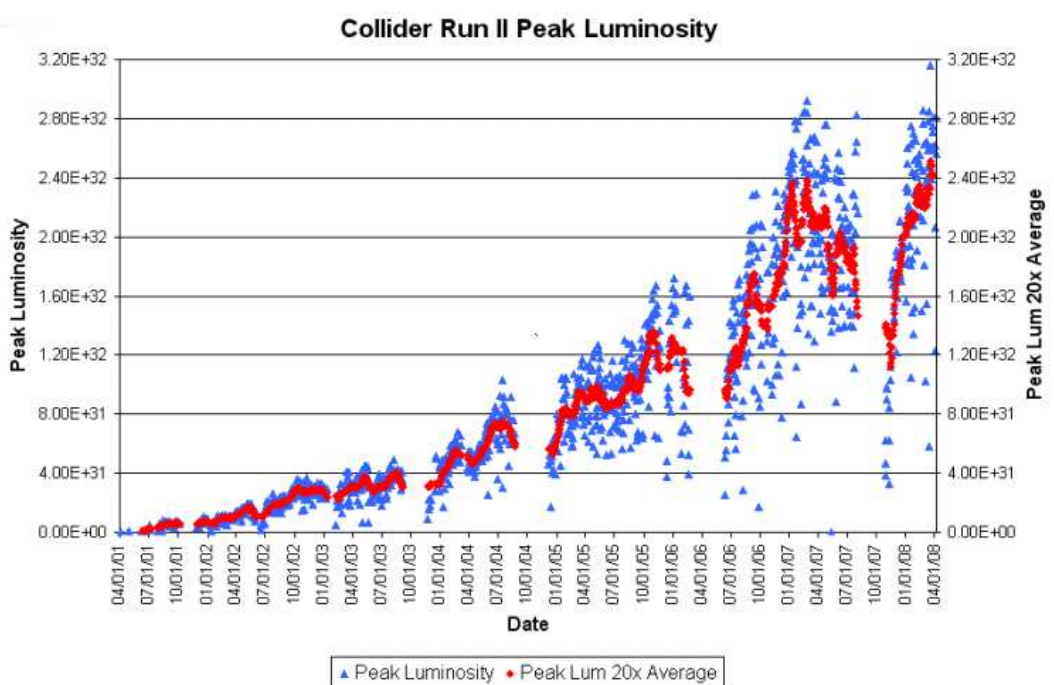

(b) Peak Instantaneous Luminosity

Figure 2.4: Luminosity at the Tevatron during Run II (2001-2008) [17]. 


\section{$2.2 \mathrm{D} \emptyset$ Detector}

The $\mathrm{D} \emptyset$ detector is a multi-purpose detector designed to identify and measure particles originating from high mass states and study large $p_{\mathrm{T}}$ phenomena. During Run I, the D $\varnothing$ experiment took about $130 p b^{-1}$ of data using the $\mathrm{D} \varnothing$ detector. For Run II, the $\mathrm{D} \varnothing$ detector was upgraded to improve detector performance and operate at higher luminosity. In this section the design and performance of the upgraded $\mathrm{D} \varnothing$ detector is described. This section is based on Reference [18] and the upgraded $\mathrm{D} \emptyset$ detector is discussed in detail in this reference.

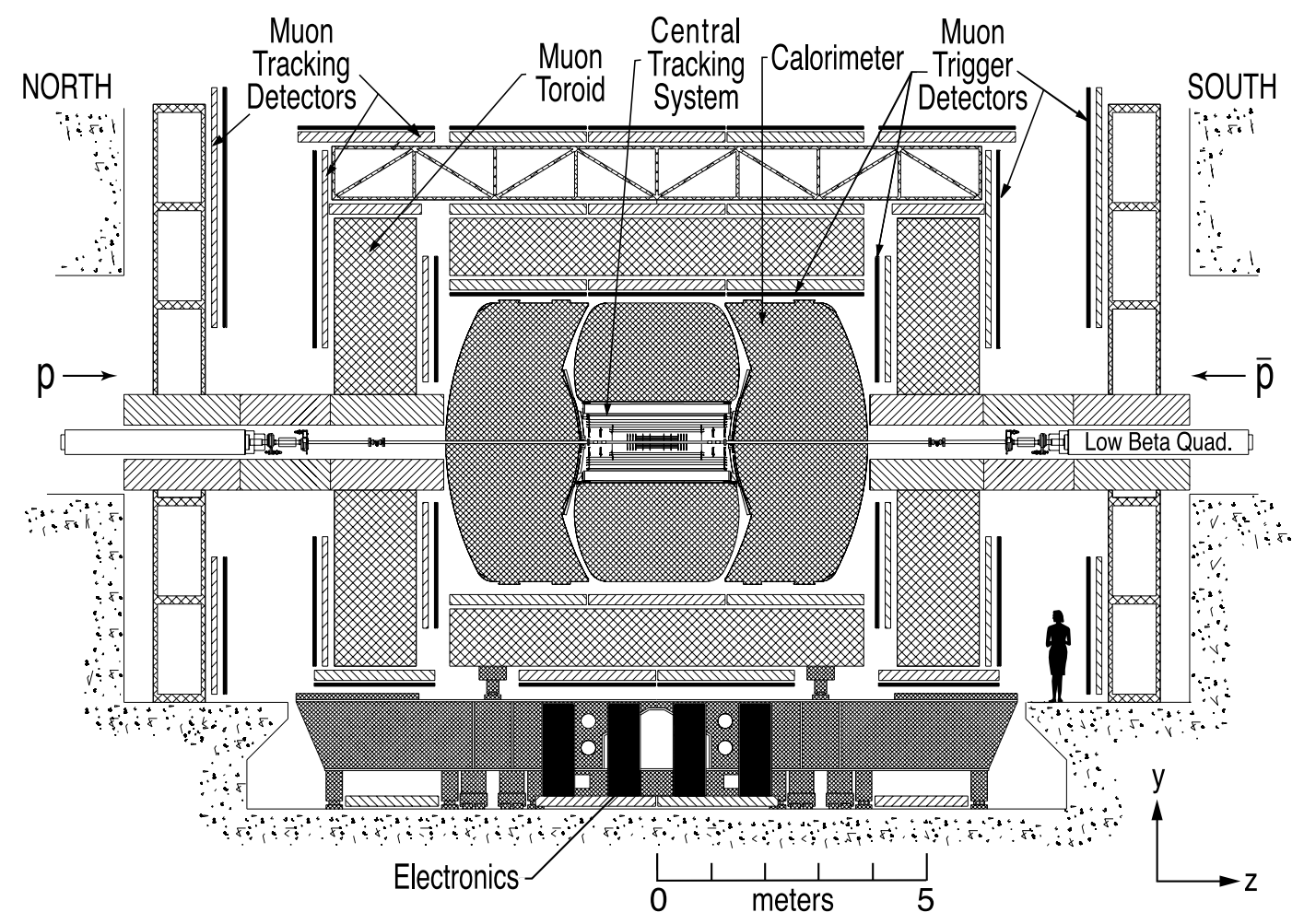

Figure 2.5: Diagram of the upgraded D $\varnothing$ detector viewed from inside the Tevatron ring.

The $\mathrm{D} \varnothing$ detector consists of several subdetectors. An overview of the $\mathrm{D} \emptyset$ detector is shown in Figure 2.5. The central tracking system that measures the vertex and the path of charged particles is described in Section 2.2.2. The calorimeters that measure the energy depositions of particles that interact electromagnetically, such as electrons and photons, but also particles that interact with the strong force, such as pions are briefly presented in Section 2.2.3. The muon detector that identifies muons is discussed in Section 2.2.4. Section 2.2.5 describes the luminosity monitor 
designed to measure Tevatron luminosity at the $\mathrm{D} \varnothing$ interaction region by counting inelastic $p \bar{p}$ collisions. Finally, Section 2.2.6 describes the trigger and data acquisition (DAQ) systems.

\subsubsection{Coordinate System}

The $\mathrm{D} \varnothing$ experiment uses a right-handed coordinate system. The $z$ axis is paralell to the beam axis, the $y$ axis is upward and the $x$ axis is outward from the center of the Tevatron. The spherical coordinate $(r, \theta, \phi)$ is also useful

$$
\left(r=\sqrt{x^{2}+y^{2}}, \theta=\arccos \left(\frac{z}{\sqrt{x^{2}+y^{2}+z^{2}}}\right), \phi=\arctan \left(\frac{y}{x}\right)\right)
$$

where the $\theta$ is the polar angle and $\phi$ is the azimuthal angle. For the polar angle, pseudorapidity $\eta$ is more convenient to use for relativistic particles and the definition is

$$
\eta=-\ln \tan \frac{\theta}{2}
$$

It is approximately the same as the true rapidity defined as

$$
y=\frac{1}{2} \ln \frac{E+p_{z}}{E-p_{z}}
$$

for finite angles in the limit that $\left(m c^{2} / E\right) \rightarrow 0$.

\subsubsection{Central Tracking System}

The central tracking system is the innermost layer of the D $\varnothing$ detector and it consists of the silicon microstrip tracker (SMT) and the central fiber tracker (CFT). A superconducting solenoidal magnet with 2.0 T magnetic field surrounds the SMT and the CFT. The SMT and the CFT measure the helical trajectories of charged particles as they travel through the tracker. They also determine

the location of the primary interaction vertex with a resolution of about $35 \mu \mathrm{m}$ along the beamline and identify b-quark jets with an impact parameter resolution better than $15 \mu \mathrm{m}$ for transverse 
momentum $p_{\mathrm{T}}>10 \mathrm{GeV} / \mathrm{c}$ at $|\eta|=0$. A schematic view of the tracking system is shown in Figure 2.6.

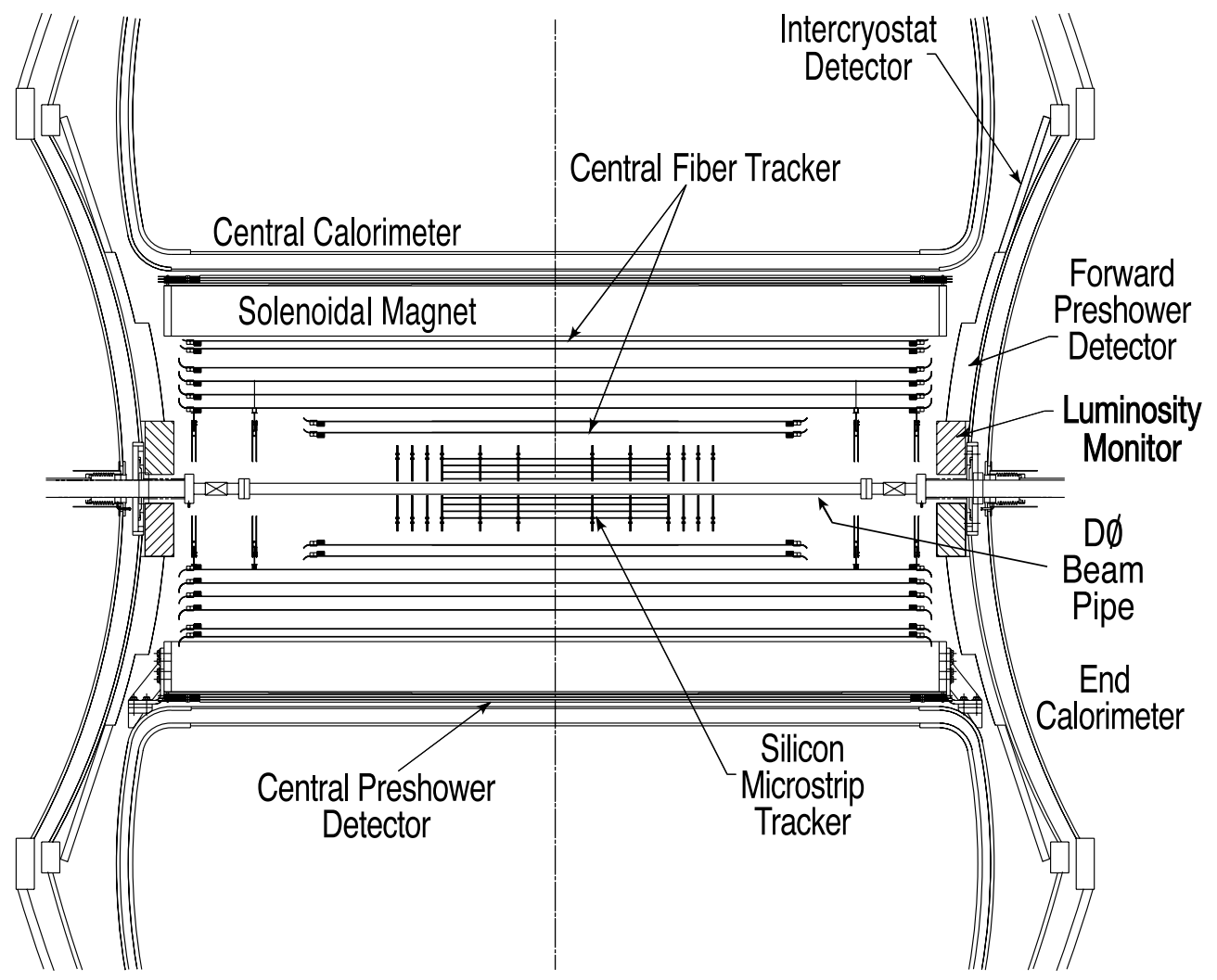

Figure 2.6: Cross-sectional view of the central tracking system with solenoid in the $x-z$ plane.

\section{Silicon Microstrip Tracker}

The SMT provides both tracking and vertexing of charged particles for almost the full $\eta$ range covered by the calorimeter and muon systems. The SMT consists of 12 F-disks, 4 H-disks and 6 barrels. The barrel detectors primarily measure the $r-\phi$ coordinate for particles in the low $\eta$ region and the disk detectors measure both $r-z$ and $r-\phi$ in the high $\eta$ region. A 3-D schematic view of the SMT is shown in Figure 2.7.

In central region of the SMT, there are 6 barrels and each barrel has 4 silicon readout layers. Layers 1 and 2 each have 12 silicon modules called "ladders." Layers 3 and 4 each have 24 ladders each, a total of 432 ladders with $2.7 \mathrm{~cm}<r<10.5 \mathrm{~cm}$ and $|z|<38 \mathrm{~cm}$. Each barrel is connected with F-disks at high $|z|$. F-disks are a set of 12 double-sided wedge modules and forward of the 3 


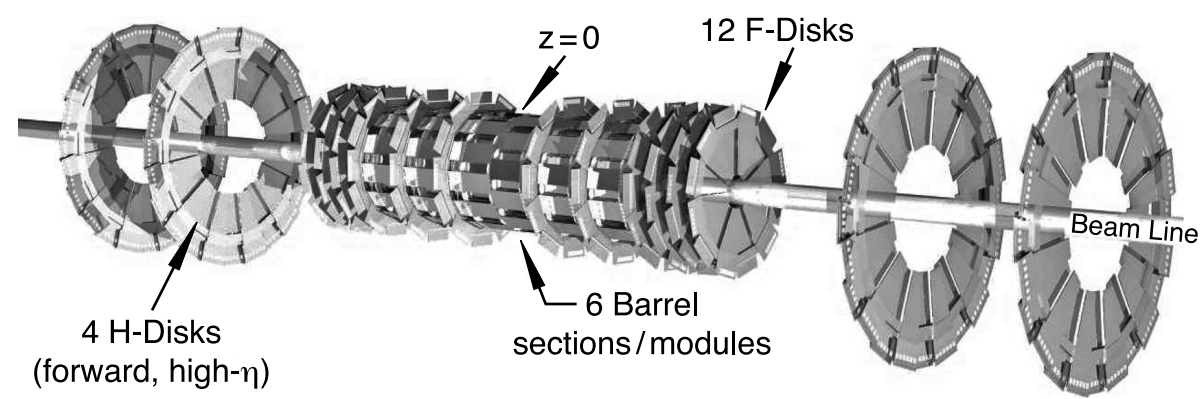

Figure 2.7: The disk and barrel design of the SMT.

disk/barrel on each side is a unit consisting of 3 F-disks. The F-disks are at $|z|=12.5,25.3,38.2$, 43.1, 48.1 and $53.1 \mathrm{~cm}$. H-disks are located in the far foward regions $(|z|=100.4,121.0 \mathrm{~cm})$ and are designed to cover the high $|\eta|$ region. They consist of 2 large-diameter disks, and on each H-disk, 24 full wedges are mounted, each full wedge consisting of two back-to-back single-sided wedges. The axial hit resolution is $10 \mu \mathrm{m}$. The $z$ hit resolution is $35 \mu \mathrm{m}$ for $90^{0}$ stereo and $450 \mu \mathrm{m}$ for $2^{0}$ stereo.

\section{Central Fiber Tracker}

The CFT is located outside of the SMT covering the radial space from 20 to $52 \mathrm{~cm}$ from the center of the beam pipe. The CFT consists of scintillating fibers mounted on eight concentric support cylinders (two inner cylinders that are $1.66 \mathrm{~m}$ long and 6 outer cylinders that are with $2.52 \mathrm{~m}$ long.) One doublet layer of fibers on each cylinder is parallel with the beam axis and the other doublet layer is at a stereo angle of $\pm 3^{\circ}$. The scintillation light provided by the scintillating fibers is carried to visible light photon counters (VLPCs) for read out through fiber waveguides. The fibers in the CFT have $835 \mu \mathrm{m}$ diameter and the CFT has a resolution of about $100 \mu \mathrm{m}$.

\subsubsection{Calorimeter}

The liquid argon calorimeters are designed to measure energy for electrons, photons, and jets including missing transverse energy $\left(E_{T}\right)$. As shown in Figure 2.8, the calorimeters can be divided into 3 parts: the central calorimeter (CC) covers $|\eta| \leq 1.0$ and the two end calorimeters (EC) extend coverage to $|\eta| \approx 4.0$. Each calorimeter contains an electromagnetic (EM) section and hadronic sections (fine and coarse). The CC and EC are located in their own cryostats held at a temperature 
of approximately $90 \mathrm{~K}$. The region between the two cryostats $(0.8<|\eta|<1.4)$ is instrumented with the intercryostat detector (ICD), which provides additional sampling layers to minimize the degradation of the energy resolution in this region.

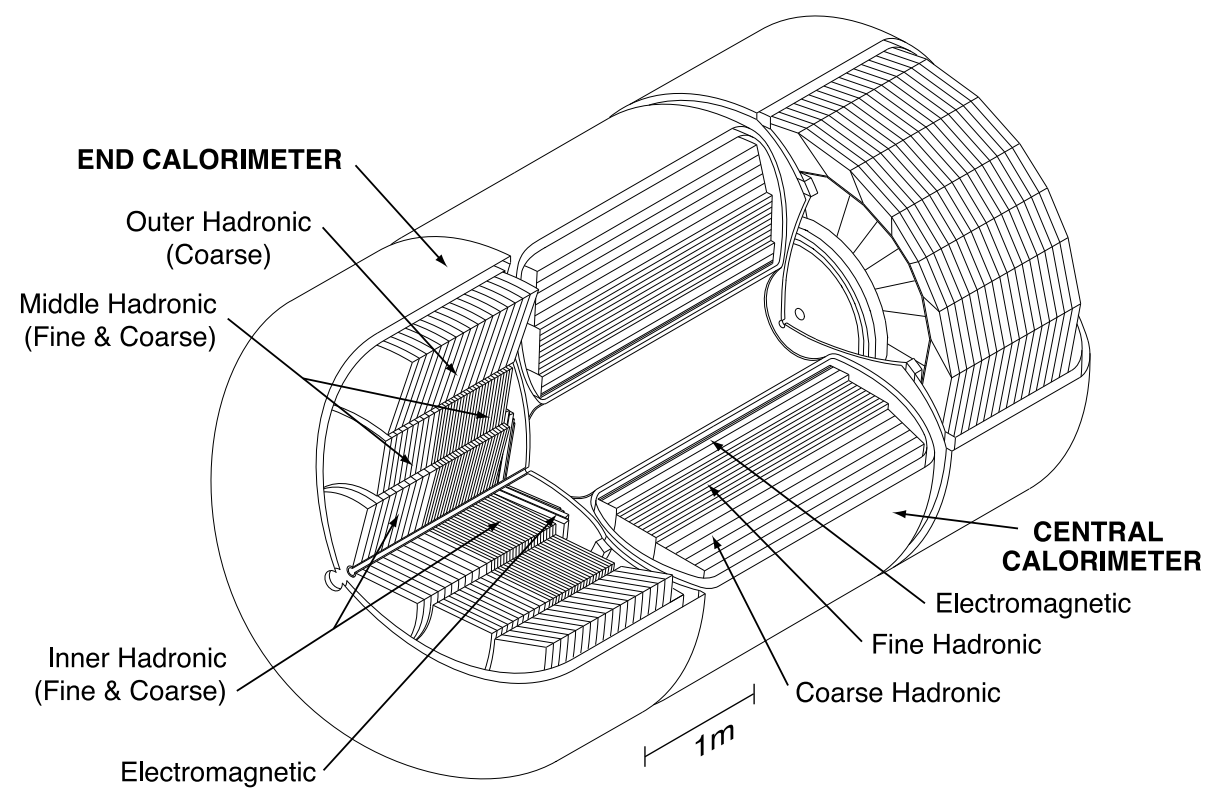

Figure 2.8: Isometric view of the central and two end calorimeters.

Thin depleted uranium absorber plates are used in the EM calorimeters ( 3 or $4 \mathrm{~mm}$ in the CC and EC, respectively). The fine hadronic (FH) calorimeters are made from $6 \mathrm{~mm}$ thick uraniumniobium $(2 \%)$ alloy plates and the coarse hadronic $(\mathrm{CH})$ calorimeters consist of $46.5 \mathrm{~mm}$ thick plates of copper in the CC or stainless steel in the EC. Figure 2.9 shows a schematic view of a calorimeter cell. This cell has an electron drift time of approximately $450 \mathrm{~ns}$ in the $2.3 \mathrm{~mm}$ liquid argon gap.

In the $\mathrm{CC}$, the cells are combined to form $4 \mathrm{EM}$ readout layers, $3 \mathrm{FH}$ layers and $1 \mathrm{CH}$ layer. In the EC, there are 4 EM layers, $4 \mathrm{FH}$ layers and $1 \mathrm{CH}$ layer. The size of the readout cells in the $\mathrm{FH}$ and $\mathrm{CH}$ layers is $\delta \eta \times \delta \phi=0.1 \times 0.1$, as shown in Figure 2.10. The EM layers have $\delta \eta \times \delta \phi=0.05 \times 0.05$ larger cell sizes are used for at $\eta>3.2$. The resolution of the calorimeter for electromagnetic particles is $\frac{14 \%}{\sqrt{\mathrm{E}}}+1 \%$ and the resolution of the calorimeter for hadronic particles is $\frac{50 \%}{\sqrt{\mathrm{E}}}+4 \%$. 


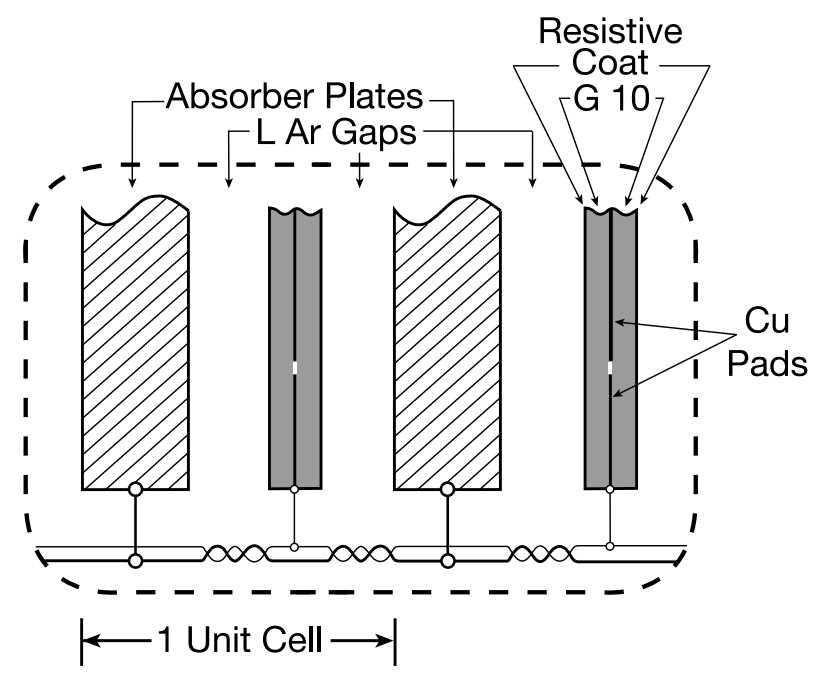

Figure 2.9: Schematic view of calorimeter cell.

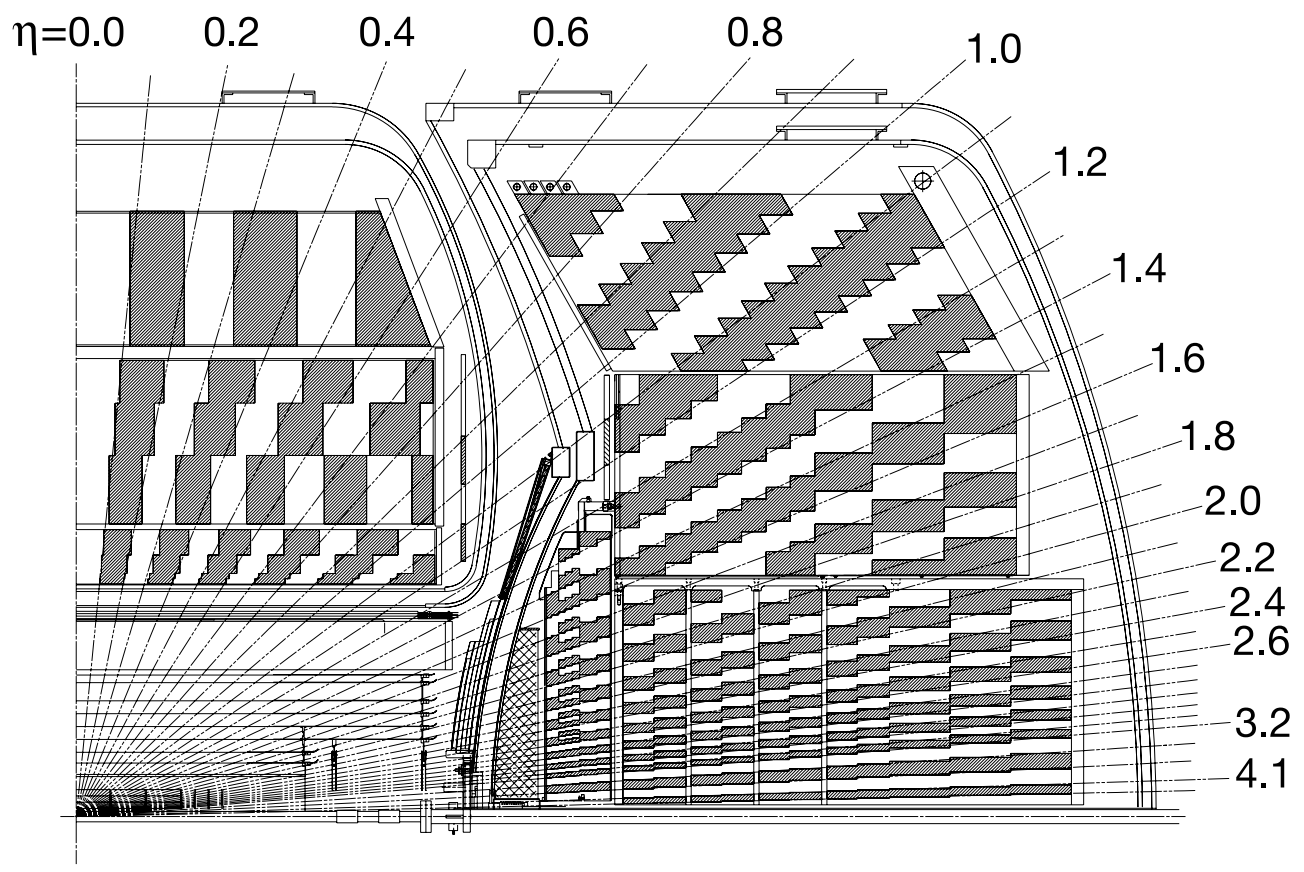

Figure 2.10: Schematic view of the $\mathrm{D} \varnothing$ calorimeter. The rays and the numbers indicate pseudorapidity intervals seen from the center of detector. 


\subsubsection{Muon Detector}

The upgraded muon system has two subdetectors, the central muon system using proportional drift tubes (PDTs) and the forward muon system using mini drift tubes (MDTs). The central muon system covers $|\eta| \leq 1.0$ and the forward muon system increases coverage to $|\eta| \approx 2.0$. Exploded views of the muon system are shown in Figure 2.11.

The central muon system consists of a toroidal magnet, drift chambers, the cosmic cap and bottom scintillation counters and the $A \phi$ scintillation counters. The toroidal magnets (visible in Figure 2.5) produce a magnetic field of approximately $1.9 \mathrm{~T}$ in the iron absorber. The drift chambers (PDTs) are arranged in three layers and they are located inside (A layer) and outside (B and C layers) of the central toroidal magnet. The PDTs record the electron drift time, giving a resolution for the drift distance in the PDTs of $\sigma \approx 1 \mathrm{~mm}$.

The forward muon system consists of end toroidal magnets $(\approx 2.0 T)$, three layers of MDTs to reconstruct muon tracks, three layers of scintillation counters to trigger on events with muons, and shielding around the beam pipe. The 3 layers of $\operatorname{MDTs}(\mathrm{A}, \mathrm{B}, \mathrm{C})$ are located inside the toroidal magnets (A), outside the toroidal magnets (B), and further out from toroidal magnets (C). The efficiency of the MDTs is $\approx 95 \%$ and the MDT coordinate resolution is about $0.7 \mathrm{~mm}$ per hit. The stand-alone momentum resolution is approximately $20 \%$ for muon momentum below $40 \mathrm{GeV} / \mathrm{c}$.

\subsubsection{Luminosity Monitor}

The luminosity monitor (LM) determines the luminosity at the $\mathrm{D} \emptyset$ detector, measures beam halo rates and makes a fast measurement of the $\mathrm{z}$ coordinate of the interaction vertex. The LM detector consists of two LM counter arrays (north and south). The LM arrays are made from 24 plastic scintillation counters with photomultiplier (PMT) readout. The LM detector has a coverage of $2.7<|\eta|<4.4$ as shown in Figure 2.12. The LM detectors are located at $z= \pm 140 \mathrm{~cm}$. The readout system for the LM detector and its calibration are discussed in detail in Appendix A.

To separate $p \bar{p}$ interactions from beam halo backgrounds, the time-of-flight for particles is mea- 


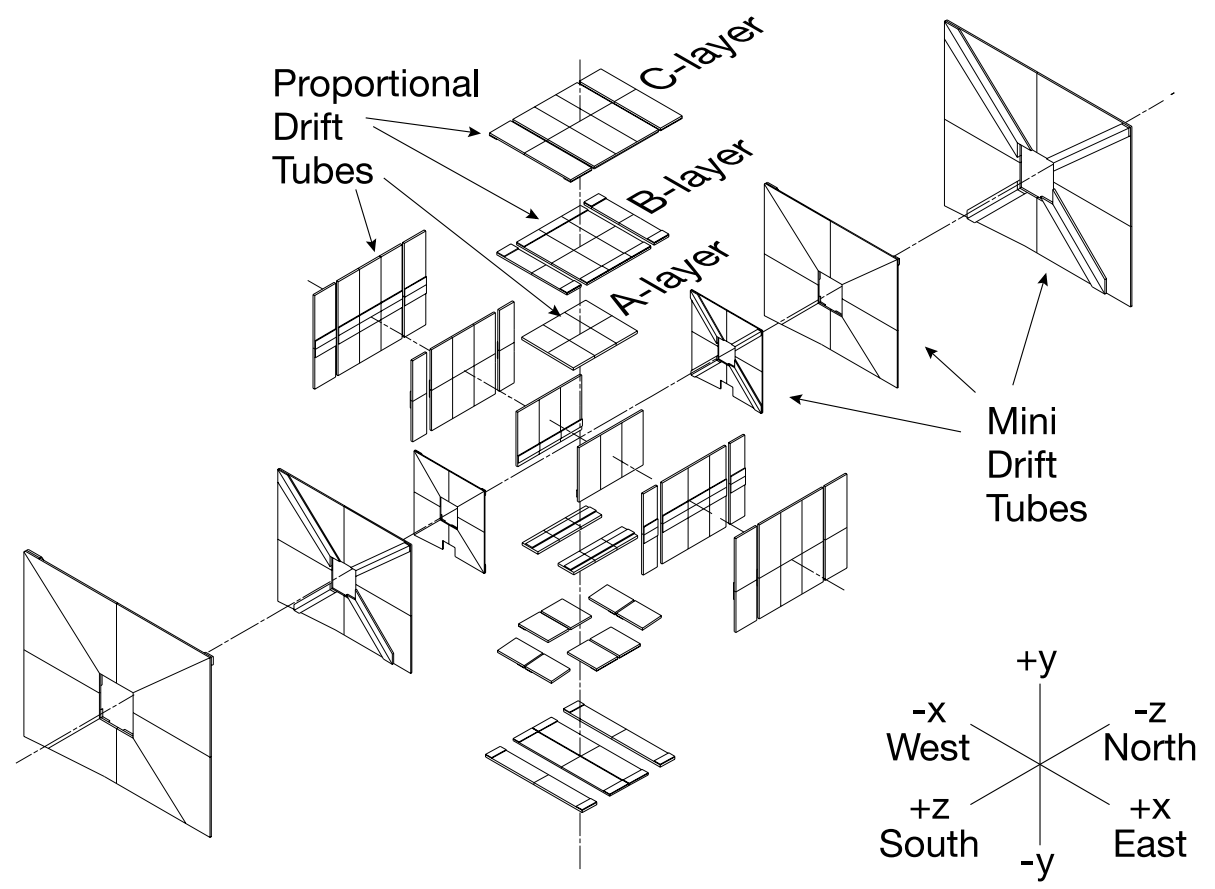

(a) Exploded view of the muon wire chambers.

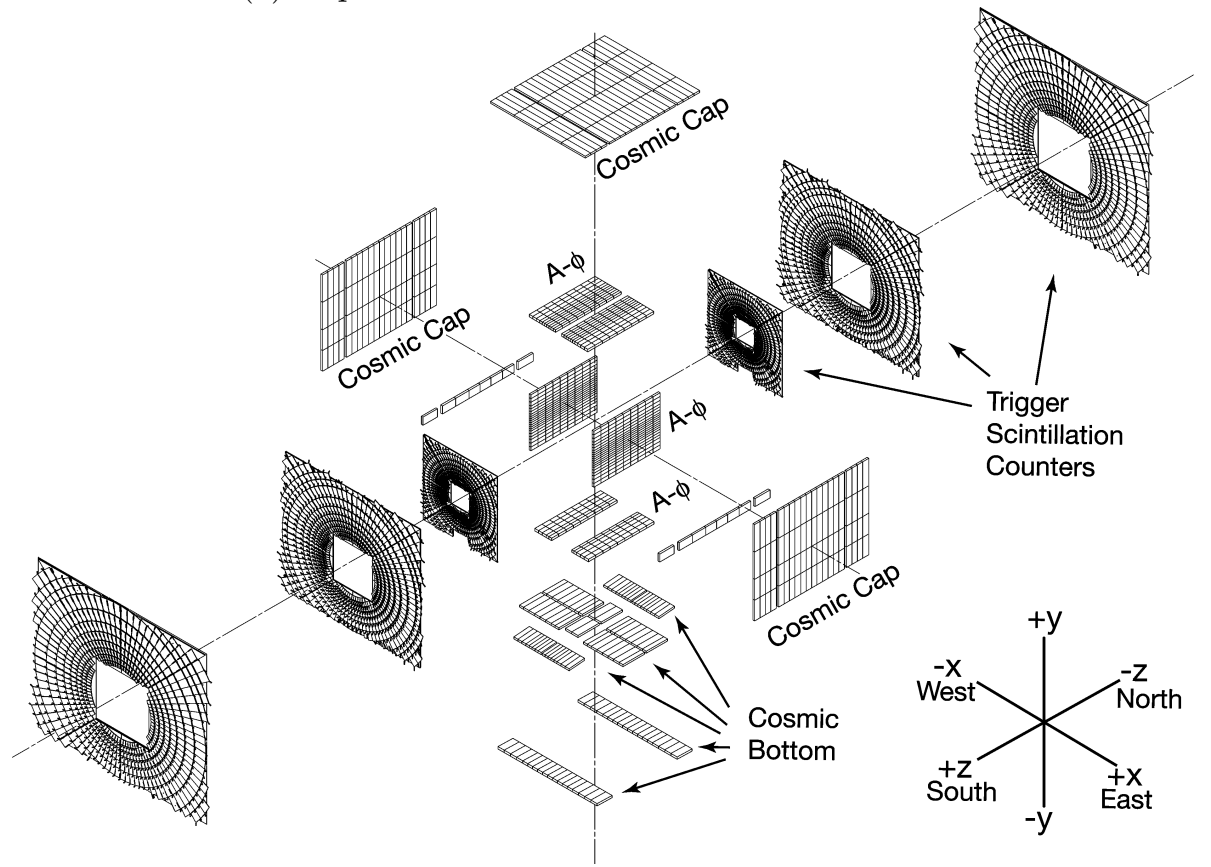

(b) Exploded view of the muon scintillation detectors.

Figure 2.11: DØ muon system. 


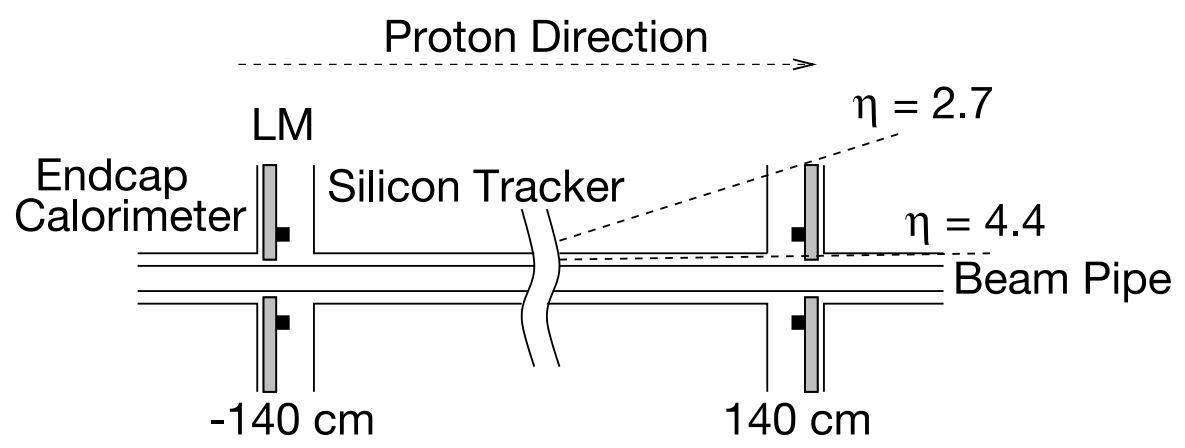

Figure 2.12: Schematic view of the LM detectors.

sured in the LM detector. The position of the interaction vertex is calculated

$$
z_{v}=\frac{c}{2}\left(t_{-}-t_{+}\right)
$$

where $t_{+}\left(t_{-}\right)$are the time-of-flight for particles hitting the south (north) LM counters and $z_{v}$ is the $z$ coordinate of the interaction vertex estimated by the LM detector. Typically, particles from beam halo have $z_{v} \approx \mp 140 \mathrm{~cm}$, and therefore $\left|z_{v}\right|<100 \mathrm{~cm}$ is required to eliminate beam halo backgrounds.

The luminosity $\mathcal{L}$ is determined from the average number of inelastic collisions per beam crossing $\left(\bar{N}_{L M}\right)$ measured by the LM detector. The formula is

$$
\mathcal{L}=\frac{f \bar{N}_{L M}}{\sigma_{L M}}
$$

where $f$ is the beam crossing frequency and $\sigma_{L M}$ is the effective cross section that takes into account the acceptance and efficiency of the LM detector.

\subsubsection{Trigger and DAQ Systems}

In the Tevatron, $p \bar{p}$ collisions happen every $396 \mathrm{~ns}$ and the initial data rate is approximately 1.7 MHz. Most of events in this stage are low $p_{\mathrm{T}}$ backgrounds, while we are interested in only high $p_{\mathrm{T}}$ physics events such as $t \bar{t}$ pair events. Furthermore, due to the limited ability of the $\mathrm{D} \emptyset$ to record and analyze data, it is necessary for the trigger system to be able to reduce the rate to about $50 \mathrm{~Hz}$. 
To do this, a three level trigger system, with the levels denoted by L1, L2 and L3, is used by DØ.

Figure 2.13 shows an overview of the D $\varnothing$ trigger systems.

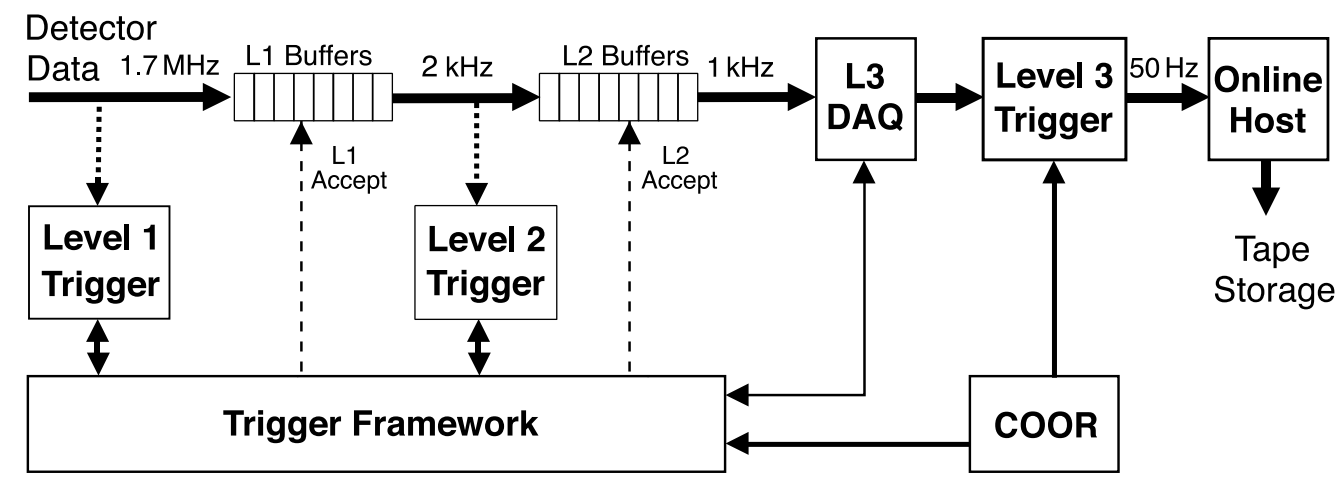

Figure 2.13: Overview of DØ trigger and DAQ systems.

\section{L1 trigger}

The L1 trigger consists of a set of hardware trigger elements and the rate of L1 acceptance is up to $2 \mathrm{kHz}$. This stage includes the calorimeter trigger (L1Cal), the muon trigger (L1Muon), and the central track trigger (L1CTT). The L1Cal decision is detemined by the energies deposited in the trigger towers covering $\delta \eta \times \delta \phi=0.2 \times 0.2$. The L1Muon trigger looks for patterns consistent with muons using the information from muon wire chambers, muon scintillation counters and tracks from the L1CTT. The L1CTT reconstructs the trajectories of charged particles and uses them to make a trigger decision. Within $\leq 3.5 \mu \mathrm{s}$, the $\mathrm{L} 1$ trigger decision should arrive at the trigger framework.

\section{L2 trigger}

The input rate of the L2 trigger is up to $2 \mathrm{kHz}$ from the L1 trigger. In this stage, the rate should be reduced to less than $1 \mathrm{kHz}$. The L2 trigger has both hardware engines and embeded microprocessors for subdetectors including tracking, calorimeter, and muon systems. In the L2 trigger, the preprocessors reconstruct physics objects and the physics objects are used individually to make trigger decisions. They are also provided to L2Global to test object correlation for trigger decision. A block diagram of the L1 and L2 trigger system is shown in Figure 2.14. 


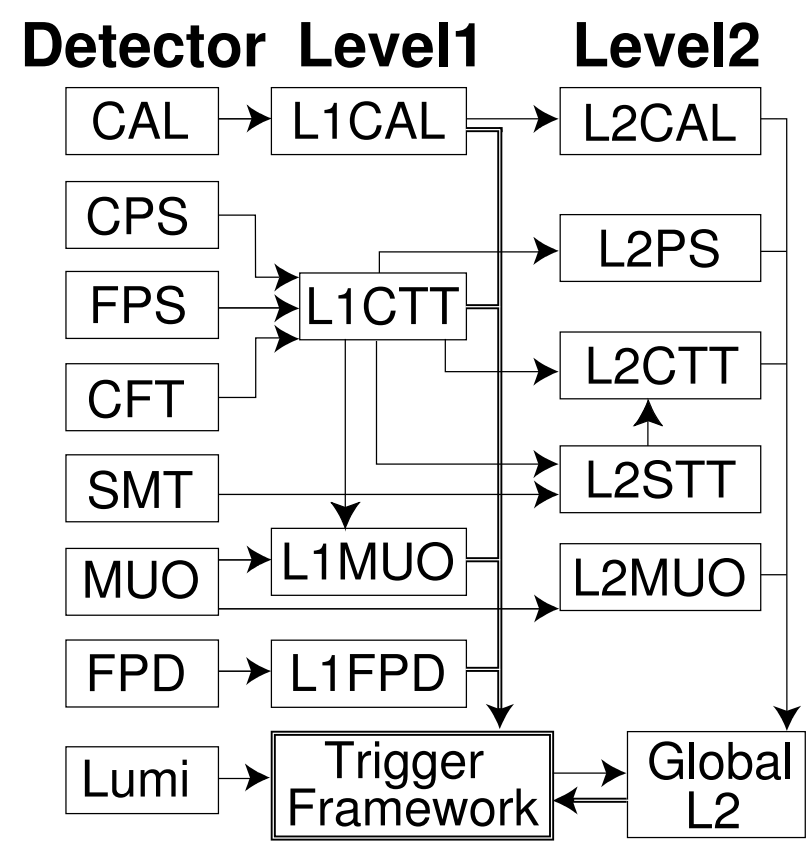

Figure 2.14: Block diagram of the L1 and L2 trigger systems. The arrows show the data flow.

\section{L3 trigger and L3DAQ}

Candidates passing through the L1 and L2 triggers are sent to the L3 trigger, and the rate is reduced to about $50 \mathrm{~Hz}$ in this stage to enable events to be recorded. The L3 trigger is a farm of microprocessors and this farm performs limited reconstruction of the physics objects in each event. In the L3 trigger, trigger decisions are constructed by not only the presence of physics objects but also the relationships between them. It takes about $15 \mathrm{~ms}$ per event for input, event building and output and about $235 \mathrm{~ms}$ per event for unpacking, reconstruction, and filtering at $1 \mathrm{kHz}$.

The L3DAQ system is in charge of sending the data for fully digitized events from the VME readout crates of subdetectors to the L3 trigger farm. The L3DAQ is designed with $250 \mathrm{MB} / \mathrm{s}$ bandwidth, corresponding to an average event size of about $200 \mathrm{kB}$ and a L2 trigger accept of rate, $1 \mathrm{kHz}$. 


\section{Chapter 3}

\section{Event Reconstruction and Monte}

\section{Carlo Simulation}

\subsection{Object Reconstruction}

In this section, the following fundamental physics objects are discussed: tracks, primary vertices, electrons, muons, jets and missing transverse energy $\left(E_{T}\right)$. The physics objects are reconstructed using the data collected by the $\mathrm{D} \varnothing$ detector or from Monte Carlo (MC) samples generated by the $\mathrm{D} \emptyset$ detector simulation. To identify the objects, specific algorithms and selections developed at $\mathrm{D} \varnothing$ are used. The b-tagging algorithm which was recently developed and is being used at $\mathrm{D} \varnothing$ is also described in this section.

\subsubsection{Tracks}

Charged particles leave their traces in the form of helical trajectories when they pass through the SMT and CFT. The SMT and CFT transform energy deposits of charged particles into electric signals called hits. In the SMT, a hit is formed by energy deposition of the charged particles in a silicon strip. The two or more adjacent hits are defined as a cluster. In the CFT, a hit is formed when a charged particle traverses a scintillating fiber. Hits from adjacent scintillating fibers 
are also clustered together. Using the hits, tracks are reconstructed by a track finding algorithm. The track finding is performed using a global track reconstruction (GTR) algorithm combining the Histogramming Track Finding (HTF) and the Alternative Algorithm (AA).

The HTF [19] is useful for the complicated cases with many hits, because this method reduces the combinations of the hits using the Hough transformation. Figure 3.1 shows an example of the Hough transformation. The hits mapped in $(x, y)$ coordinates are transformed into $(\rho, \phi)$ by the Hough transformation. Hits from a track candidate produce a peak in the $\phi-\rho$ histogram, while random combinations of hits will be distributed uniformly. This result is parameterized with templates. The templates are futher processed by a 2D Kalman filter in order to discard fake templates and calculate track parameters precisely.

(a)
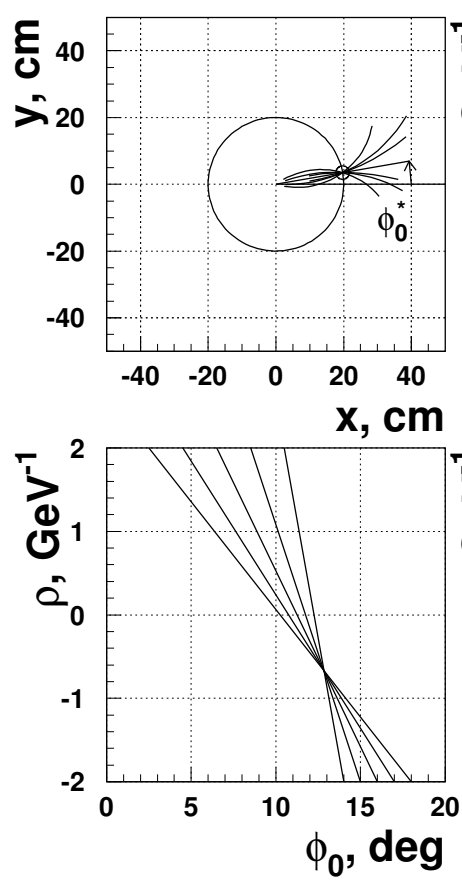

(c) (b)
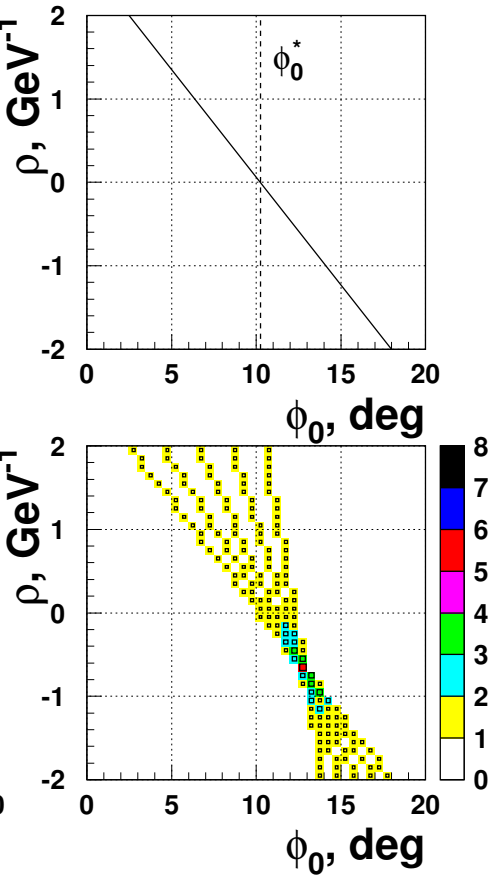

(d)

Figure 3.1: Illustration for an example of Hough transformation. This example is for $1.5 \mathrm{GeV}$ muon track. (a) The family of trajectories containing a given hit. (b) The geometric place of all trajectories containing a given hit in parameter space. (c) Curves from different hits intersect at one point corresponding to the track parameters. (d) The point of intersection can be seen as a peak in the $(\rho, \phi)$ histogram $[19]$.

The AA [20, 21] is based on pattern recognition and its main concerns are keeping the track 
reconstruction efficiency high and reducing the rate of fake tracks. The AA can reconstruct low momentum tracks as well as high momentum tracks. In the AA, all possible combinations of 3 hits in the SMT layers are constructed at the beginning stage and track candidates are extrapolated to hits in the next layer of the SMT or CFT. If the $\chi^{2}$ cut is satisfied, it is associated with the track candidate. The AA also allows "CFT only" tracks to be reconstructed if hits in the SMT are less than 3 .

\subsubsection{Primary Vertices}

The primary vertex $(\mathrm{PV})$ is defined as the location of the inelastic $p \bar{p}$ interaction within the DØ detector, and it is used as the origin in calculating kinematic variables used in physics analyses. The adaptive primary vertex algorithm [22] is currently being used at D $\varnothing$. The reconstruction using this algorithm consists of 3 stages: track selection, vertex fitting and vertex selection.

In the adaptive primary vertex algorithm, tracks are required to have $p_{\mathrm{T}}>0.5 \mathrm{GeV} / \mathrm{c}^{2}$ as well as 2 or more hits in the SMT. Only tracks within $2 \mathrm{~cm}$ are clustered, called as z-clustering, to discard tracks generated by other interactions. All tracks selected by the z-clustering algorithm are fitted to the same vertex and both the location and other information for the vertex are determined. The tracks with the largest $\chi^{2}$ are removed until the $\chi^{2}$ is less than 10 . Then, the distance of closest approach (dca) to the previously fitted vertex is calculated for tracks and $d c a / \sigma(d c a)<5$ is required. The tracks that pass the algorithm are fitted to a common vertex using the adaptive fitting algorithm. In the adaptive fitting, errors on tracks are reweighted by the following function

$$
w_{i}=\frac{1}{1+e^{\left(\chi_{i}^{2}-\chi_{\text {cutoff }}^{2}\right) / 2 T}}
$$

where $\chi_{i}^{2}$ is the $\chi^{2}$ contribution of track $i$ to the vertex, $\chi_{\text {cutoff }}^{2}$ is the distance where the weight function drops to 0.5 and $\mathrm{T}$ is a parameter that determines the sharpness of the function. Finally, the hard-scatter vertex is selected by the minimum bias probability selection algorithm. 


\subsubsection{Electrons}

Electrons [23] deposit their energies in the calorimeter and provide tracks in the central tracking system. Therefore, electrons are reconstructed with information from two detectors. Energy clusters in the calorimeter are reconstructed and matched to the track reconstructed by the central tracking system. This track matching is also useful for distinguishing electrons from photons since photons do not leave track in the central tracking system.

The simple cone algorithm makes use of a list of towers with energy deposits sorted by $E_{\mathrm{T}}$. In the list, a seed is defined and initialized with the highest $E_{\mathrm{T}}$ tower. Then all of towers with $\Delta R<0.2$ are added to the seed. The final group of towers is an EM cluster. Various parameters, including track matching, are calculated for each EM cluster to help identify electrons. The important parameters are described below.

- Isolation:

$$
f_{i s o}=\frac{E_{t o t}(\Delta R<0.4)-E_{E M}(\Delta R<0.2)}{E_{E M}(\Delta R<0.2)}
$$

where $E_{E M}(\Delta R<0.2)$ is the EM energy with $\Delta R<0.2$ (based on EM layers) and $E_{\text {tot }}(\Delta R<$

0.4 ) is the EM energy with $\Delta R<0.4$ (based on EM, FH and $\mathrm{CH}$ layers). $f_{\text {iso }}<0.15$ is required in this analysis.

- EM fraction:

$$
E M F=\frac{E_{E M}(\Delta R<0.2)}{E_{t o t}(\Delta R<0.2)} .
$$

This is the ratio of EM calorimeter energy to the hadron calorimeter energy. Large EM fraction $(E M F>0.9)$ is required for EM clusters in this analysis.

- Track matching:

$$
\chi^{2}=\left(\frac{\Delta \phi}{\sigma_{\phi}}\right)^{2}+\left(\frac{\Delta z}{\sigma_{z}}\right)^{2}
$$


where $\Delta \phi(\Delta z)$ is the difference in $\phi(z)$ coordinate between the location of the EM cluster in outermost EM calorimeter layer and the extrapolation of the track to the same layer. $\chi^{2}<50$ is required in this analysis.

- Electron Likelihood:

$$
\mathcal{L}(x)=\frac{P_{s i g}(x)}{P_{s i g}(x)+P_{b g}(x)}
$$

where $P_{s i g}\left(P_{b g}\right)$ is the probability for EM clusters to be signal (background). It is calculated with 7 variables: EM fraction, H-matrix, ratio of the calorimeter transverse energy of the cluster to the transverse momentum of the matched track, track matching $\chi^{2}$ probability, distance of closest approach, number of tracks in a $\Delta R<0.05$ cone, and sum of the $p_{\mathrm{T}}$ of all tracks. $\mathcal{L}>0.85$ is applied to the high quality electrons only.

\subsubsection{Muons}

Muons [24] can be reconstructed using information from both the muon detector and the central tracking system. In the muon detector, local muons, which are reconstructed using only muon detector information, are identified with high purity. The wire hits in each (A, B, C) layer are jointed to form track line segments, and the segments are matched with the scintillator hits. Then, the reconstructed segments from the three layers are used to measure the muon momentum using the curvature caused by the toroidal magnet. The resolution on the muon momentum is poor, so the local muon is matched to a track from the central tracking system to improve the resolution. A successfully matched local muon is called a central track-matched muon.

Reconstructed muons are classified by two parameters: muon type and muon quality. nseg is defined to sort muon types. Muons with only one layer hits in the muon detector have $|n s e g|=1$, muons with hits in the $\mathrm{B}$ or $\mathrm{C}(\mathrm{BC})$ layer have $|n s e g|=2$, and muons with $\mid$ nseg $\mid=3$ have hits in both A and BC layers. A local muon segment not matched to a central track has a negative nseg. The second parameter is muon quality. There are three muon qualities: "Loose", "Medium", and 
"Tight". In this analysis, Medium muon quality with $n s e g=3$ is used. The selection criteria for this quality is,

- at least two A layer wire hits

- at least one A layer scintillator hit

- at least two BC layer wire hits

- at least one BC scintillator hit (except for central muons with less than four BC wire hits).

The central track matching between local muons and tracks from the central tracking system considers the following characteristics: the number of hits in the central tracking system, $\chi_{\text {dof }}^{2}$ of the central track fitting, and the dca in $(\mathrm{x}, \mathrm{y})$ of the track with respect to the primary vertex. In this analysis, $|d c a|<0.2 \mathrm{~cm}$ is required and it tightens $|d c a|<0.02 \mathrm{~cm}$ if the track has hits in the SMT. $\chi_{d o f}^{2}<4$ is also required.

Additional requirements for muon identification are muon isolaton and the cosmic ray veto. The muon isolation cut is designed to remove backgrounds from heavy flavor muon decay such as $b \rightarrow \mu$. To avoid these backgrounds, $\Delta R(\mu$, jet $)>0.5$ is required for a muon to be isolated from a nearby jet. Cosmic ray backgrounds are removed using the scintillator hit times and the requirement that $\mid$ ABC-layer time $\mid<10 \mathrm{~ns}$.

\subsubsection{Jets}

Quarks and gluons hadronize to jets of particles through the strong interaction, and the resulting hadrons shower in the calorimeter. The reconstruction and measurement of jets are very important to physics analyses such as top physics and Higgs search that include them in the final state, where the accurate measurement of the jet momentum is required to infer the original quarks and gluons. The T42 algorithm [25] and improved legacy cone algorithm (ILCA) [26] are used in Run II to reconstruct jets.

The T42 algorithm attempts to keep low energy cells of coming from the signal and reject the cells from background. The threshholds are $4 \sigma$ and $2 \sigma$ in this algorithm, where $\sigma$ is the RMS of the 
measured pedestal distribution of the cell. Cells passing $4 \sigma$ threshold are believed to originate from signal. Cells passing $2 \sigma$ threshold are left if they are neighbors to cells believed to originate from signal and otherwise they are rejected. When the $4 \sigma$ cut is applied, only less than 1.5 noisy cell per event will be left after the cut.

After noisy cells are removed, the most energetic calorimeter towers, where the tower is sum of cells in $\delta \eta \times \delta \phi=0.1 \times 0.1$, with greater than $0.5 \mathrm{GeV}$ are selected by the ILCA. This is called "seeds". Then, all of towers around the seed with $\Delta R<0.5$ in the $\eta \times \phi$ dimension are added to the seed and it is defined as a jet if the jet energy is more than $1 \mathrm{GeV}$. The $E_{\mathrm{T}}$ weighted centeroids are calculated in each cone and used as the center of a cone. This procedure is repeated until the cone axis corresponds to the centeroid. The final jet $E_{\mathrm{T}}$ requirement is $E_{\mathrm{T}}>6 \mathrm{GeV}$. If two jets overlap, they are merged if the overlapping energy is greater than half of each jet energy, otherwise they are split into two individual jets. Following a split or merge, the $E_{\mathrm{T}}$ and the axis of jets are recalculated.

\section{Jet Energy Scale}

The reconstructed jet needs a "Jet Energy Scale" (JES) correction [28, 29] to measure more precisely for the jet momentum. The goal of the JES correction is to make the jet energy reconstructed by the calorimeter match on average sum of particle energies for particles in the jet before passing through the detector.

The JES correction is defined in Equation 3.6

$$
E_{\text {jet }}^{\text {particle }}=\frac{E_{j e t}^{r a w}-O}{F_{\eta} \times R \times S}
$$

where quantities used in the equation are defined as follows

- $E_{\text {jet }}^{\text {particle }}$ : corrected jet energy

- $E_{j e t}^{r a w}$ : uncorrected jet energy,

- $O$ : offset energy correction 
- $F_{\eta}$ : relative response correction ( $\eta$-intercalibration)

- $R$ : absolute response correction

- $S$ : showering correction.

The underlying events from beam remnants and multiple parton interaction make energy deposits in the calorimeter jet cone called the "offset energy". These energy deposits are not associated with hard scattering, and thus are not associated with the jet. The "offset energy correction" removes the average energy from this effect. It is determined using minimum-bias events triggered by the luminosity monitor detector, and these minimum-bias events are assumed to be representative of inelastic $p \bar{p}$ collisions. The energy density per tower for minimum-bias events is measured as a function of pseudorapidity and for various primary vertex multiplicities in order to account for the instantaneous luminosity dependence, shown in Figure 3.2.

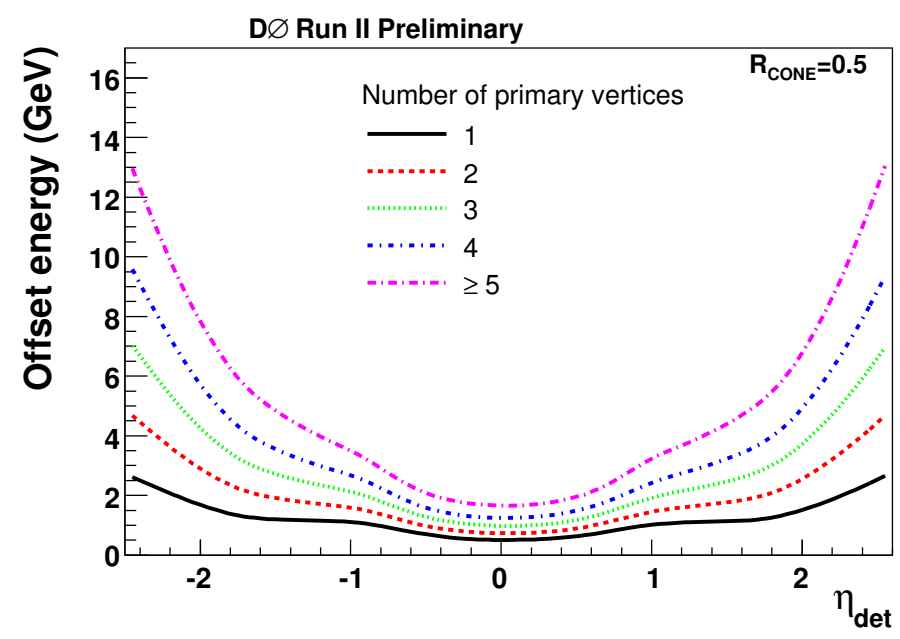

Figure 3.2: Offset energy of $\Delta R<0.5$ cone jets for various primary vertex multiplicites [28].

The "relative response correction" and "absolute response correction" have the purpose of making the calorimeter have the same response independent of energy. The calorimeter is not well instrumented between the $\mathrm{CC}$ and EC calorimeters, causing a non-uniform response in this region. The ICD detector provides additional sampling layers between the CC and EC, but it is not sufficient. To completely remove this effect, the relative response correction is performed using the $E_{T}$ Projection Fraction (MPF) method explained in Figure 3.3. The MPF method measures the 
differences in response between jet (probe) objects and photon (tag) objects. The correction is obtained in the $|\eta|<0.1$ region with photon+jets and dijet events. After calibrating the dijet events against the photon+jet events, the measurement is extended to the whole kinematic range using the dijet events. The absolute response correction is measured using the MPF method for photon+jets events applying the offset and relative response corrections. Figure 3.4 shows the measured absolute response for the $\Delta R<0.7$ jet cone size. The response in each pseudorapidity region is consistent after the relative response correction.

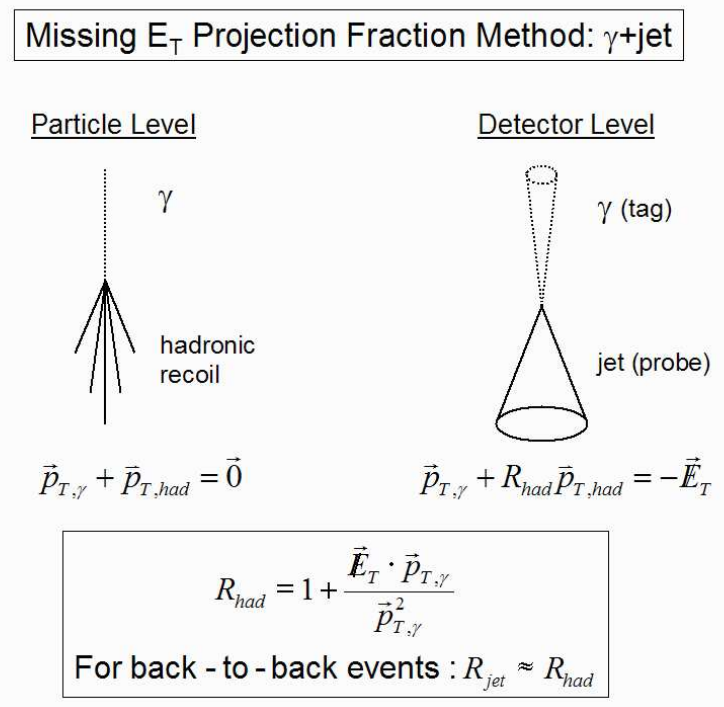

Figure 3.3: The MPF method description [28].

The showering correction is to correct for instrumental effects such as shower development in the calorimeter and magnetic field bending. In the Monte Carlo, the ratio between the energies of inside and outside jet cone is measured ("detector+physics") and the same ratio is measured in the generation level without detector effects ("physics only"). Then the ratio of "detector+physics" to "physics only" is defined as the showering correction. To evaluate this correction, photon+1 jet events are used with the requirement of exactly one primary vertex. Figure 3.5 shows the measured showering correction split into jet $\eta$ regions. 


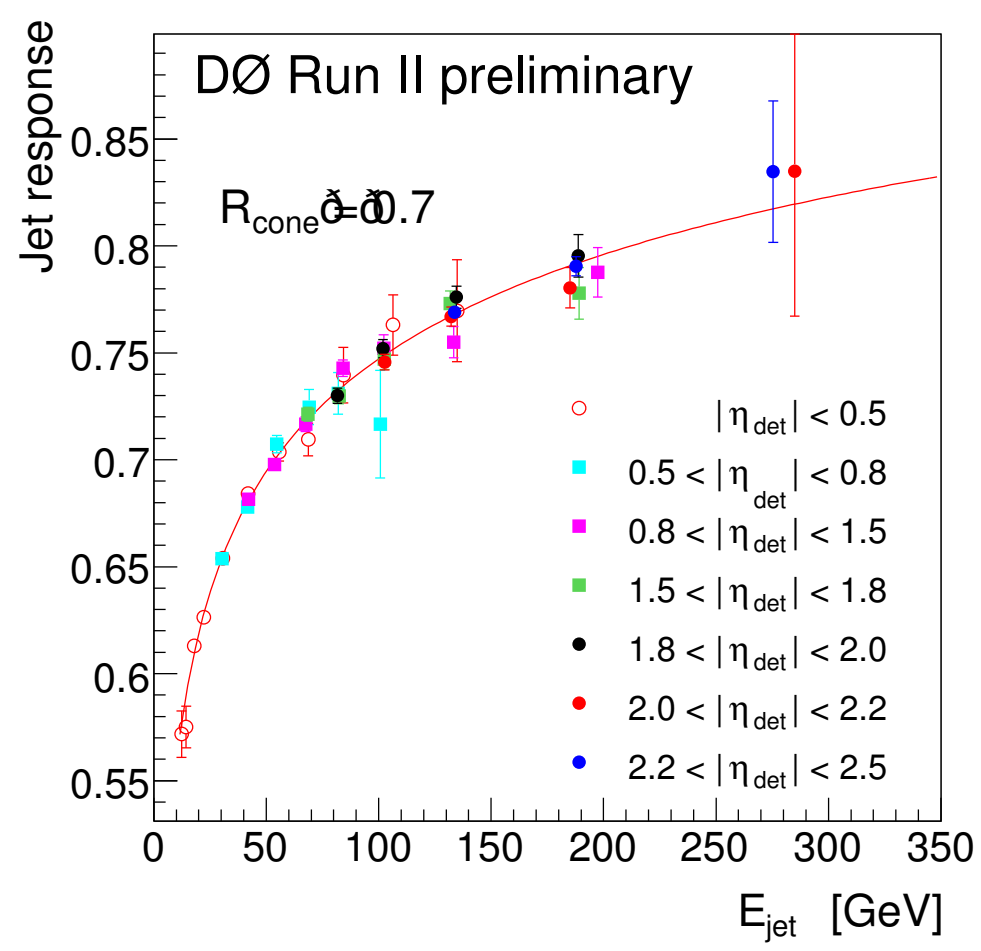

Figure 3.4: The measured absolute response results for various $\eta$ regions as a function of partially corrected jet energy [28].

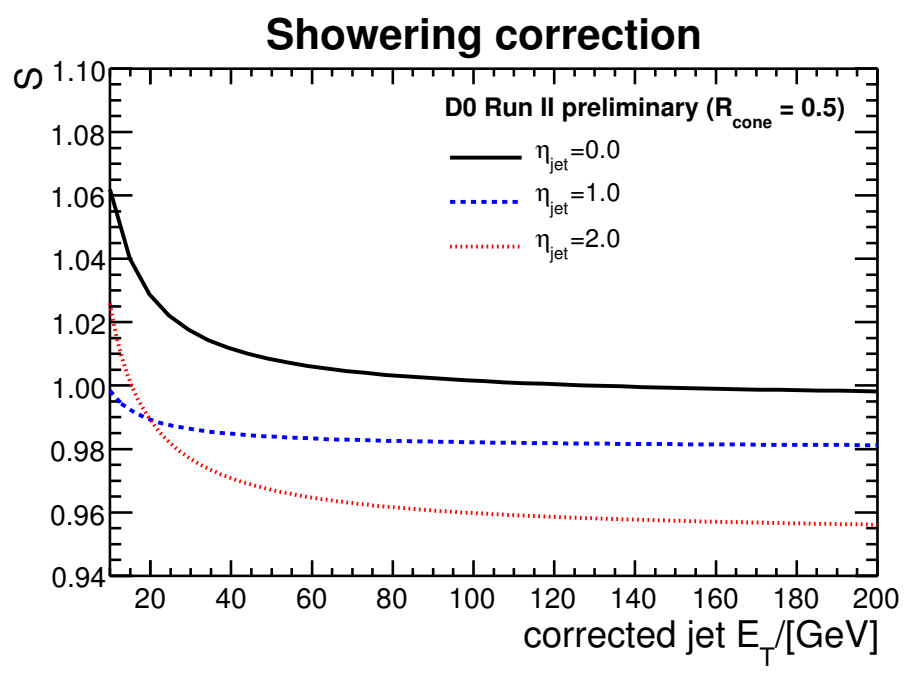

Figure 3.5: The measured showering correction for $\Delta R<0.5$ jet cone in data as a function of corrected jet $E_{\mathrm{T}}[28]$. 


\subsubsection{Missing Transverse Energy}

There is undetected energy in the detector due to non-interacting particles such as neutrinos. The quantity defined as missing transverse energy $\left(E_{T}\right)[30]$ is calculated to describe the effect from these invisible particles. The momentum imbalance resulting from invisible particles can be estimated in the transverse plane only because the detector does not cover the whole forward region including the beam pipe. $\mathbb{E}_{T}$ is reconstructed by vectorially summing the energy of cells in the electromagnetic and hadronic calorimeter and correcting for the momentum of muons that exit the calorimeter.

\subsection{7 b-tagging}

The event signature for many physics analyses in the $\mathrm{D} \emptyset$ experiment, such as $t \bar{t}$ pair and Higgs events have a b-jet in the final state. If the b-jet can be identified with high efficiency, the backgrounds in these physics analyses can be suppressed significantly and the sensitivity for searches and precision of measurements will increase. Therefore, it is very important to maximize the efficiency for b-jet identification, called b-tagging. The b-tagging algorithm can be classified by the characteristics of the b-quark: lifetime tagging and soft lepton tagging.

b-hadron has a long life time [3] because of small $\mathrm{V}_{\mathrm{cb}}$ and $\mathrm{V}_{\mathrm{ub}}$. Tagging based on the long life time is called "lifetime tagging" and there are 3 different types of lifetime tagging: Counting Signed Impact Parameters (CSIP) tagger [31], Jet Lifetime Probability (JLIP) tagger [32] and Secondary Vertex Tag (SVT) tagger [33]. The DØ experiment combines the 3 different lifetime taggers using a neural network and trains to optimize b-tagging. This is called the Neural Network (NN) tagger [34] and the efficiency for b-tagging improves about $15 \%$ compared to the other taggers.

Another characteristics of the b-quark is that it can decay semi-leptonically. Approximately $20 \%$ of b-jets have a lepton (muon) in the final state and a jet can therefore be identified as a b-jet if a lepton (muon) is found inside the jet. This is called muon-tagging, and is done by the "Soft Lepton (Muon) Tag" (SLT) tagger [35]. This SLT tagger was recently combined with the neural network lifetime method to maximize the tagging efficiency and reduce the fake rate after muon-tagging. It is called the SLTNN tagger [36]. In this section, the NN tagger based on CSIP, JLIP and SVT is 
briefly described. The SLTNN tagger will be discussed in detail in Appendix B.

The NN tagger uses 7 quantites from CSIP, JLIP and SVT as NN input variables. The definition of variables are described in Table 3.1. A NN is constructed using the input variables, NN training is

\begin{tabular}{|c|c|c|}
\hline \hline Rank & Variable & Description \\
\hline 1 & SVTdls & Decay length significance of the secondary vertex \\
2 & CSIPcomb & Weighted combination of the tracks' impact parameter significances \\
3 & JLIPprob & Probability that the jet originates from the primary vertex \\
4 & SVT $\chi_{d o f}^{2}$ & $\chi_{d o f}^{2}$ of the secondary vertex \\
5 & SVTntracks & Number of tracks used to reconstruct the secondary vertex \\
6 & SVTmass & Mass of the secondary vertex \\
7 & SVTnum & Number of the secondary vertices found in the jet \\
\hline \hline
\end{tabular}

Table 3.1: NN input variables ranked in order of power [34].

performed, and the operating points are determined by the output of NN training. Then, b-tagging efficiency is measured in both MC and data samples. The scale factor is calculated to correct the difference between them. To measure the b-tagging efficiency in data sample, the system 8 method [37] is used in DØ. The fake rate is estimated using the negative tag rate (NTR). The system8 and NTR methods are described in Appendix B. Figure 3.6 shows the plot of b-tagging efficiency versus fake rate.

\subsection{Monte Carlo Simulation}

The Monte Carlo technique is a method of calculating integrals in a multi-dimensional space by random sampling [38]. It allows a detailed simulation of detector response to simulated data. This section describes how the detector is modeled and MC samples are generated at $\mathrm{D} \emptyset$. The corrections to the MC simulations are also discussed in this section.

\subsubsection{Monte Carlo Generation at DØ}

The generation of a $p \bar{p} \quad \mathrm{MC}$ event at $\mathrm{D} \varnothing$ is performed by simulating the following chain: the hard scatter interaction, underlying event, hadronization, DØ detector simulation and finally event reconstruction. 


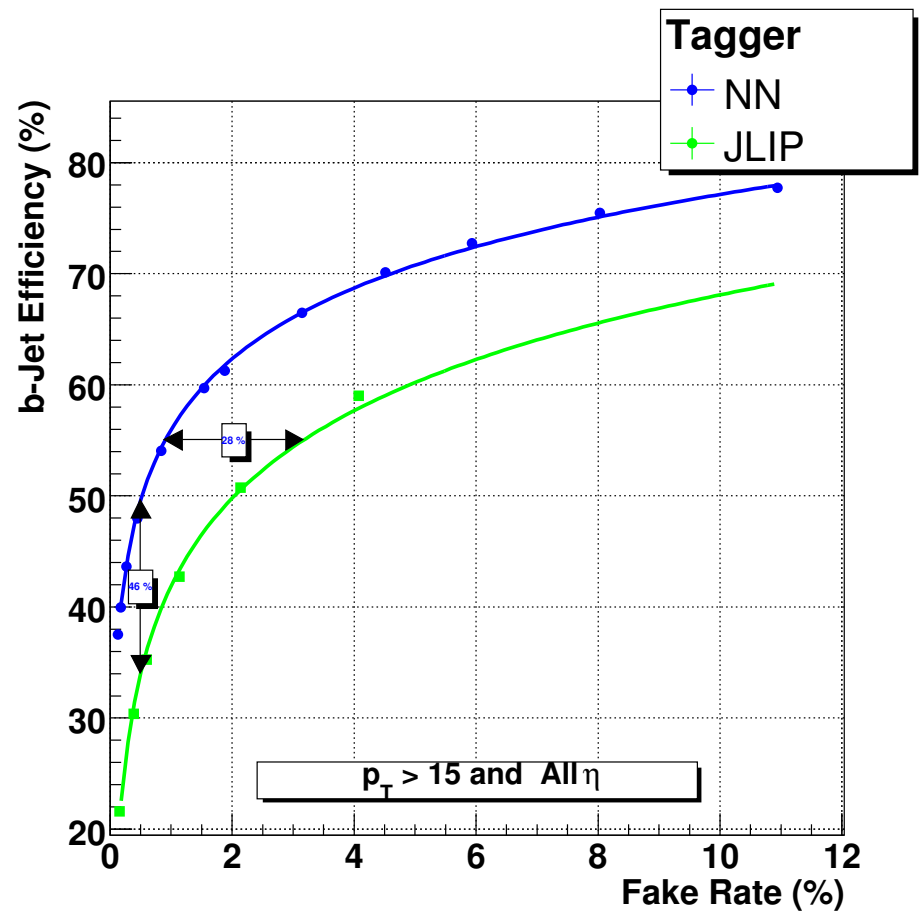

Figure 3.6: Efficiency vs. Fake rate plot for the NN tagger and the JLIP tagger [34].

The hard scatter interaction is defined as the perturbative QCD process generated using a calculation of the leading order matrix element. The ALPGEN [39] generator is typically used with the CTEQ6L1 [40] including the NLO correction used for the PDF. The underlying event is defined as everything except for the hard scattering and it consists of the "beam-beam remnant" plus initial and final state radiations. Also, multiple parton interactions contribute to the underlying event. PYTHIA 6.323 [41] is used to model the underlying event. Then, the partons simulated by the above generators are hadronized into colorless mesons and baryons. This hadronization describes the strong interaction effects and is performed by PYTHIA.

After hadronization, the $\mathrm{D} \emptyset$ detector response is simulated for the particles in the final state. The $\mathrm{D} \emptyset$ detector simulation is performed by two independent software packages: d0gstar and d0sim. d0gstar is a wrapper for the full GEANT simulation [42] and it simulates the detector response using the $\mathrm{D} \emptyset$ detector geometry and materials. Figure 3.7 shows a simulated event in d0gstar. Then $d 0$ sim performs electronics simulation (digitization) plus the overlay of additional minimum bias interactions from the output of d0gstar. Noise from each subdetector is simulated and added 
in this stage. The simulated MC events passing from $d 0$ sim, as well as real data collected by DØ detector, are called "raw data". The "raw data" from MC and real data are treated and reconstructed identically in the event reconstruction algorithms which are discussed in section 3.1.

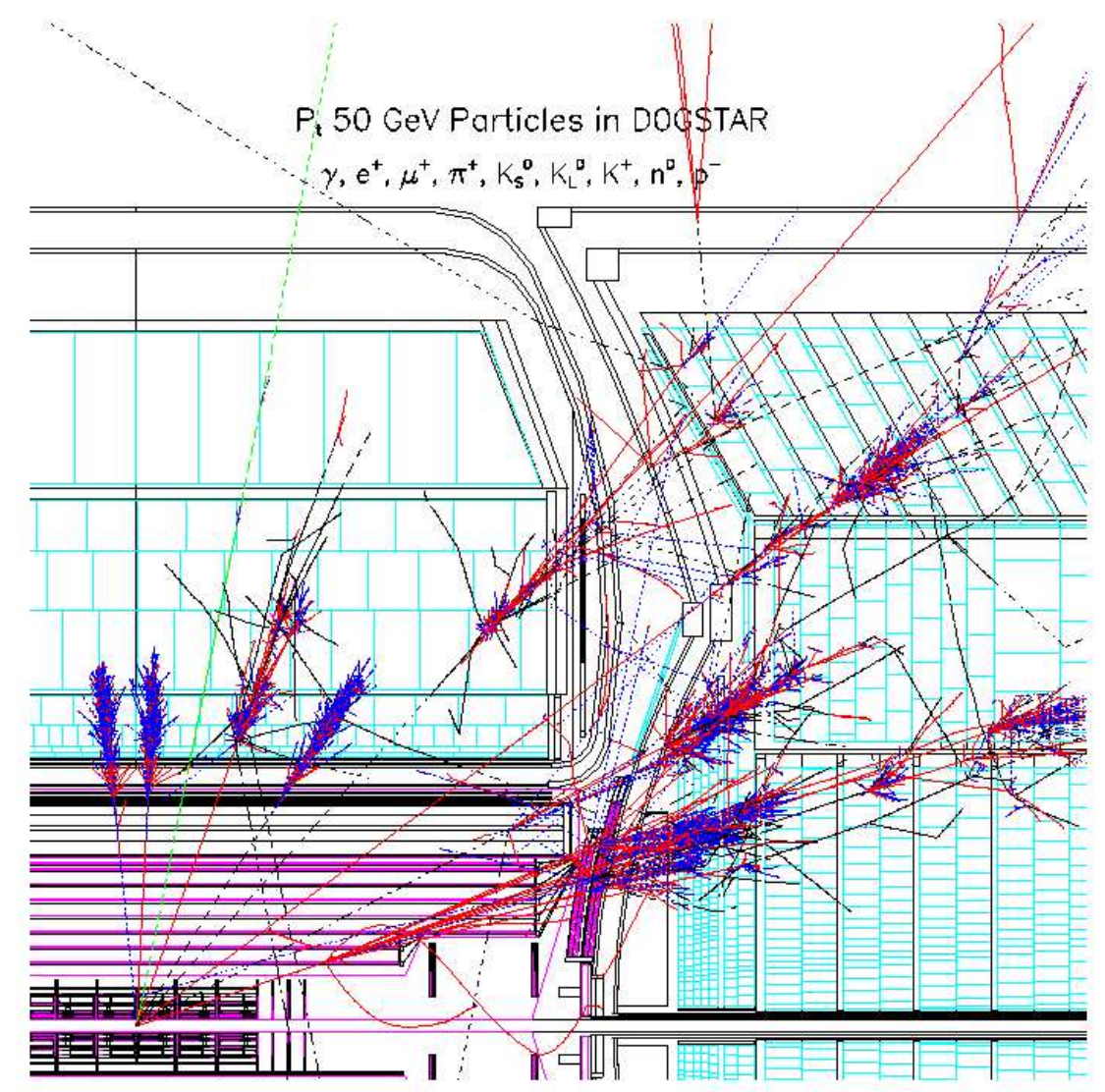

Figure 3.7: A MC event with $50 \mathrm{GeV} p_{\mathrm{T}}$ particles simulated in d0gstar [43].

\subsubsection{Correction}

In spite of much effort, the MC simulation does not perfectly model the DØ detector. Typically, the detector resolution in the MC simulation is underestimated and corrections are needed to take into 
account for the difference between data samples and MC simulations. The scale factors between them are also measured in event reconstruction level. In this section, the MC corrections for various physics objects are described.

\section{Electrons}

For electrons, two different scale factors are measured using $Z \rightarrow e e$ data and MC samples: one is for electron reconstruction and another is for the track matching plus likelihood cut. The two scale factors are then multiplied. Electron reconstruction efficiencies in both data and MC samples are shown in Figure 3.8 as a function of jet $p_{\mathrm{T}}$. The top plot in the Figure 3.8 (a) shows the reconstruction efficiencies in both data and $\mathrm{MC}$ as a function of jet $p_{\mathrm{T}}$. The bottom plot in the Figure 3.8 (a) shows the measured scale factor between data and MC reconstruction efficiencies. It is measured only in the $\mathrm{CC}$ region $(|\eta|<1.1)$. Using the reconstructed electrons in $Z \rightarrow e e$ samples, track matching + likelihood cut efficiency is also measured and the scale factor is calculated. Figure 3.8 (b) shows the result of this correction. The two scale factors are multiplied and used as a MC correction for the electron efficiency.

\section{Muons}

Muons simulated by the MC need both smearing and correction factors. Muon smearing is applied

to have a similar resolution for data and MC muons. The smearing is done in the variable $\frac{q}{p_{\mathrm{T}}}$, where $q$ is the charge of muon track. The following function is applied for the smearing correction [24]

$$
\frac{q}{p_{\mathrm{T}}} \rightarrow \frac{q}{p_{\mathrm{T}}}+\left(A+\frac{B}{p_{\mathrm{T}}}\right) \times R n d
$$

where $R$ nd is a Gaussian random number generated with width 1 and mean value 0 . The smearing parameters $\mathrm{A}$ and $\mathrm{B}$ are determined using the following 3 different samples at $p_{\mathrm{T}}=40 \mathrm{GeV}$ :

- Muons with SMT hits and $\left|\eta_{C F T}\right|<1.6$

- Muons with SMT hits and $\left|\eta_{C F T}\right|>1.6$ 

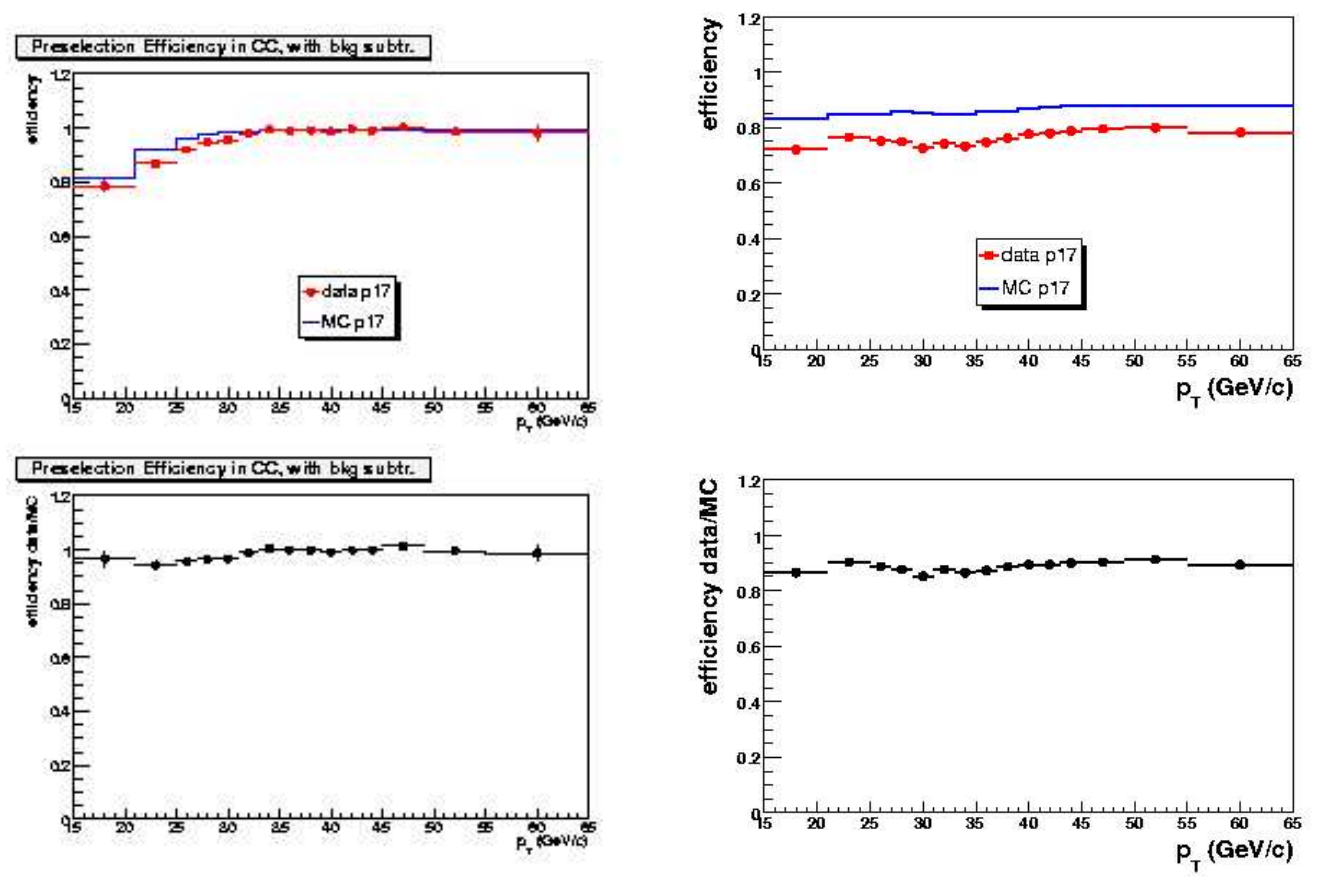

(a) Reconstruction efficiency

(b) Track-matching+likelihood cut efficiency

Figure 3.8: Electron reconstruction efficiency for data (red) and MC (blue) $Z \rightarrow e e$ samples in (a) top. Bottom plot of (a) is the scale factor for electron reconstruction. Plots in (b) show the track matching and liklihood cut efficiencies (top) of data (red) and MC (blue) as well as the scale factor (bottom) [23]. 
- Muons without SMT hits.

After applying muon smearing, the correction factors can be determined for muon reconstruction, track matching and isolation individually. Each correction factor is measured by the ratio of data efficiency to MC efficiency. The total correction is the product of 3 correction factors.

$$
S F=\left(\frac{\varepsilon_{\text {data }}^{r e c o}}{\varepsilon_{M C}^{r e c o}}\right) \times\left(\frac{\varepsilon_{\text {data }}^{\text {trk }}}{\varepsilon_{M C}^{\text {trk }}}\right) \times\left(\frac{\varepsilon_{\text {data }}^{\text {iso }}}{\varepsilon_{M C}^{\text {iso }}}\right)
$$

Each efficiency is determined using $Z \rightarrow \mu \mu$ data and MC samples. In Figure 3.9, the plot on top shows the scale factor for muon reconstruction as a function of muon $\eta$. The scale factors for track-matching (middle) and isolation (bottom) are also shown in Figure 3.9.

\section{Jets}

There exist differences between the simulated MC samples and the real data samples for jet energy scale, jet energy resolution, and jet reconstruction efficiency. These three differences are correlated with each other and therefore corrections should be applied in a consistent way. A method called JSSR (Jet Shifting + Smearing + Removing) is applied to jets [44, 45].

The formula, $\Delta S=\frac{p_{\mathrm{T}^{j e t}}-p_{\mathrm{T}^{\gamma}}}{p_{\mathrm{T}^{\gamma}}}$, is measured in $\gamma+$ jets events for both data and MC samples. The measured $\Delta S$ is plotted as a function of the $\gamma p_{\mathrm{T}}$ distribution and fitted with a gaussian $\times$ turn-on shown in Equation 3.9

$$
f(\Delta S)=N \times\left(1+\operatorname{erf}\left(\frac{\Delta S-\alpha}{\beta \sqrt{2}}\right)\right) \times e^{-\frac{\left(\Delta S-\Delta S_{0}\right)^{2}}{2 \sigma^{2}}}
$$

where the turn-on function is a model for the effect of the inefficiency to reconstruct low jet $p_{\mathrm{T}}$. The turn-on function models jet reconstruction in efficiency, the mean value of the Gaussian $\left(\Delta S_{0}\right)$ models differences in the jet energy scale, and the width of the Gaussian $(\sigma)$ models differences in jet $p_{\mathrm{T}}$ resolution. Figure 3.10 shows the $\Delta S$ distributions for each jet $\eta$ region before (left) and after (right) applying the corrections. 

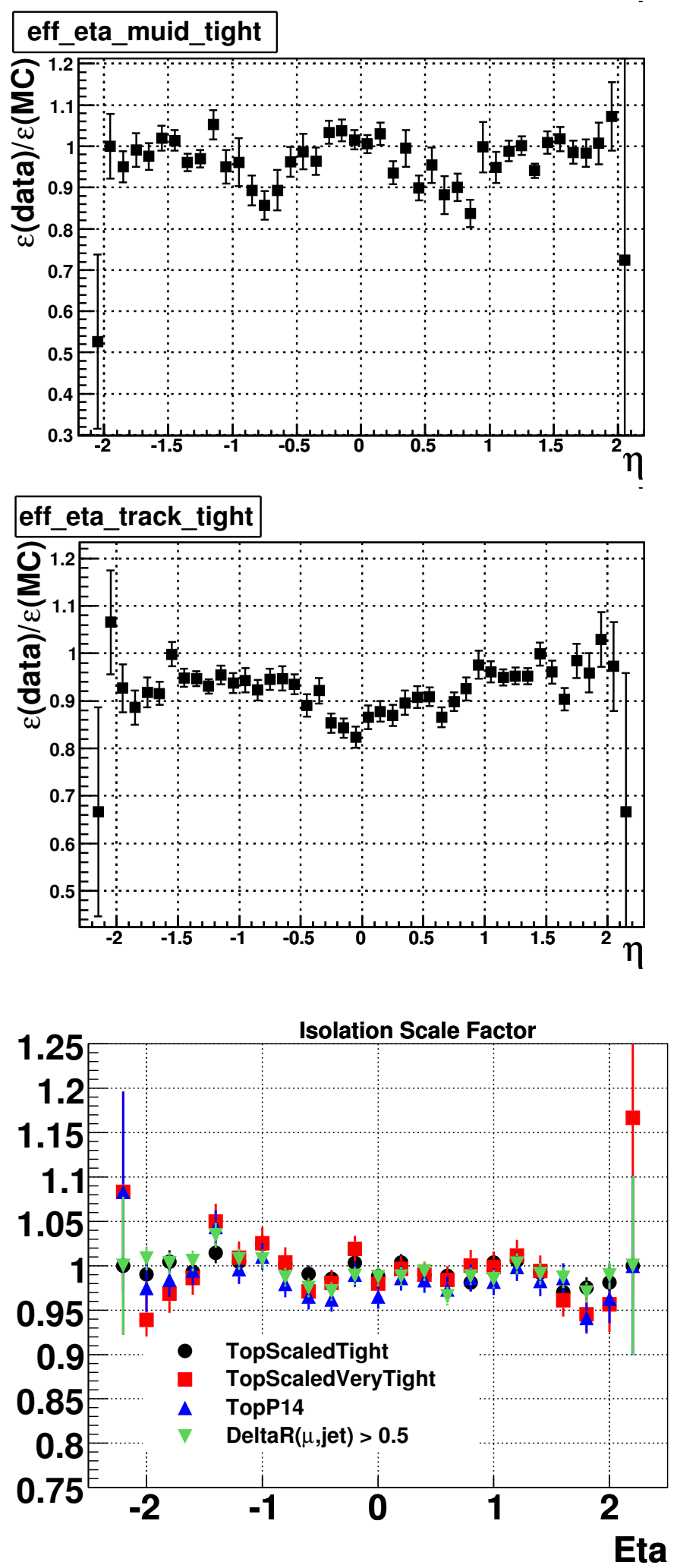

Figure 3.9: Ratio of data and MC efficiencies for muon reconstruction (top), muon track-matching (middle) and muon isolation (bottom) [24]. In the bottom plot, the green is the scale factor for muon isolation. 

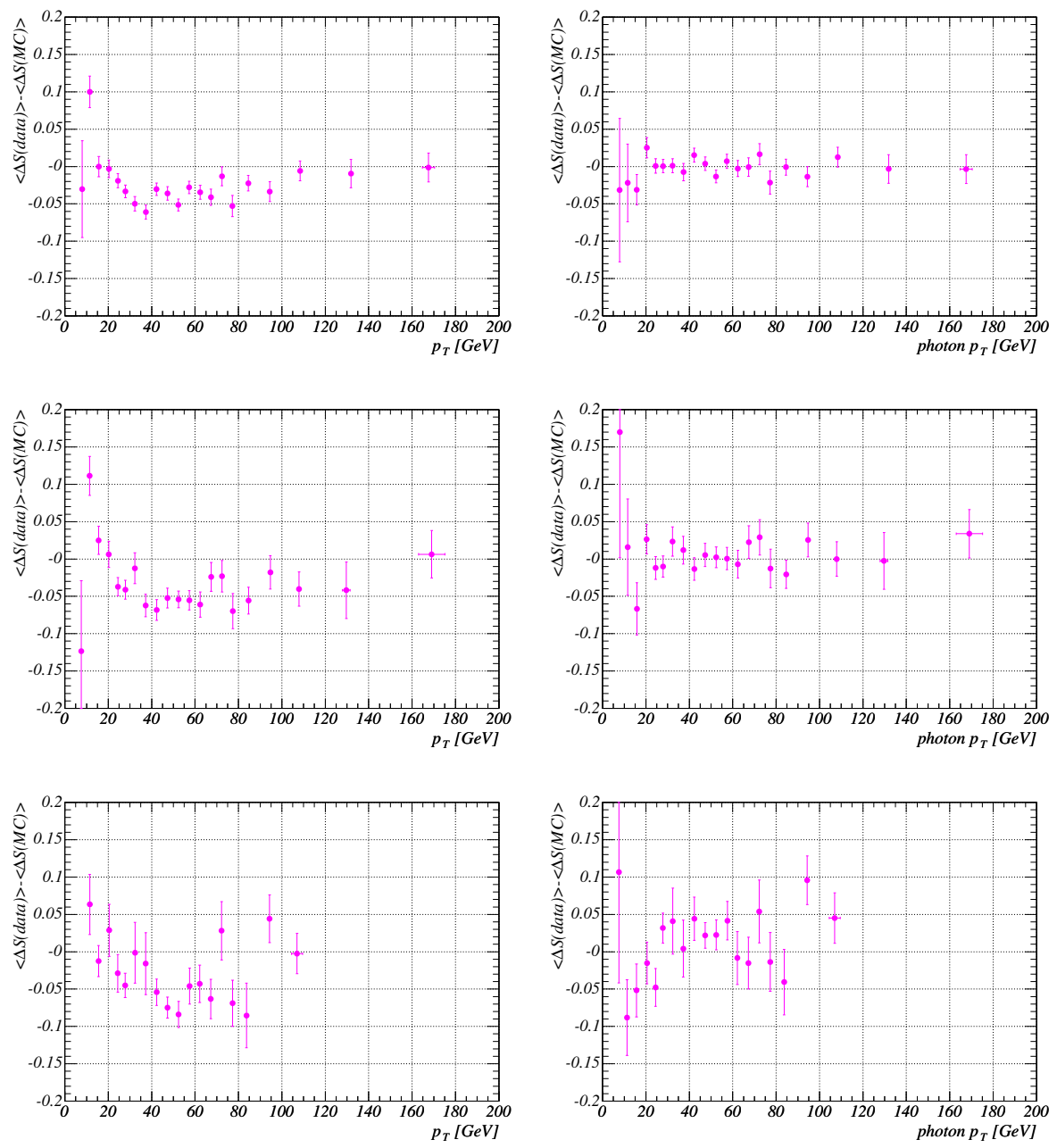

Figure 3.10: Distributions of $\langle\Delta S\rangle_{\text {data }}-\langle\Delta S\rangle_{M C}$ before (left) and after (right) applying corrections to the MC jets in CC (top), ICR (middle), and EC (bottom) $\eta$ region [44].

\section{b-tagging}

The b-id group provides a Tag Rate Function (TRF) that gives the estimated tagging efficiency and fake rate. The b-jet tagging efficiency is measured on both data and MC samples respectively. The difference between data and MC is large and the scale factor between them is measured to correct this difference. Figure 3.11 shows b-tagging efficiency in MC (red line) and data (green line) and the scale factor between them (blue line) on jet $p_{\mathrm{T}}$ (left) and $\eta$ (right) distributions.

The b-jet tagging efficiency, scale factor between data and MC samples and fake rate are determined as a function of jet $p_{\mathrm{T}}$ and $\eta$ dependences. Then, the TRF for a b-jet is computed by 

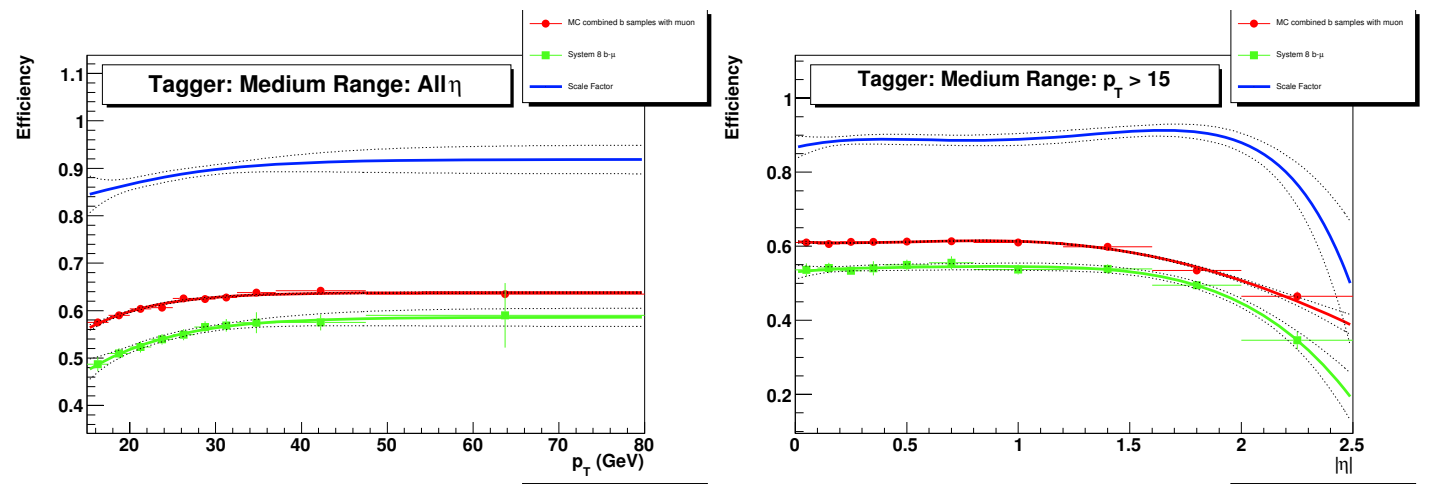

Figure 3.11: b-tagging efficiency in MC (red line) and data (green line) and the scale factor between them (blue line) with $1 \sigma$ error bands (black dashed line) on jet $p_{\mathrm{T}}$ (left) and $\eta$ (right) distributions [34].

multiplying the inclusive b-taging efficiency in MC by the scale factor as follows

$$
\begin{gathered}
T R F_{b}=(\text { scale factor }) \times\left(\varepsilon_{b-\text { tagging }} \text { in } M C\right) \\
T R F_{c}=(\text { scale factor }) \times\left(\varepsilon_{c-\text { tagging }} \text { in } M C\right) .
\end{gathered}
$$

Figure 3.12 shows the TRF for b-jet.

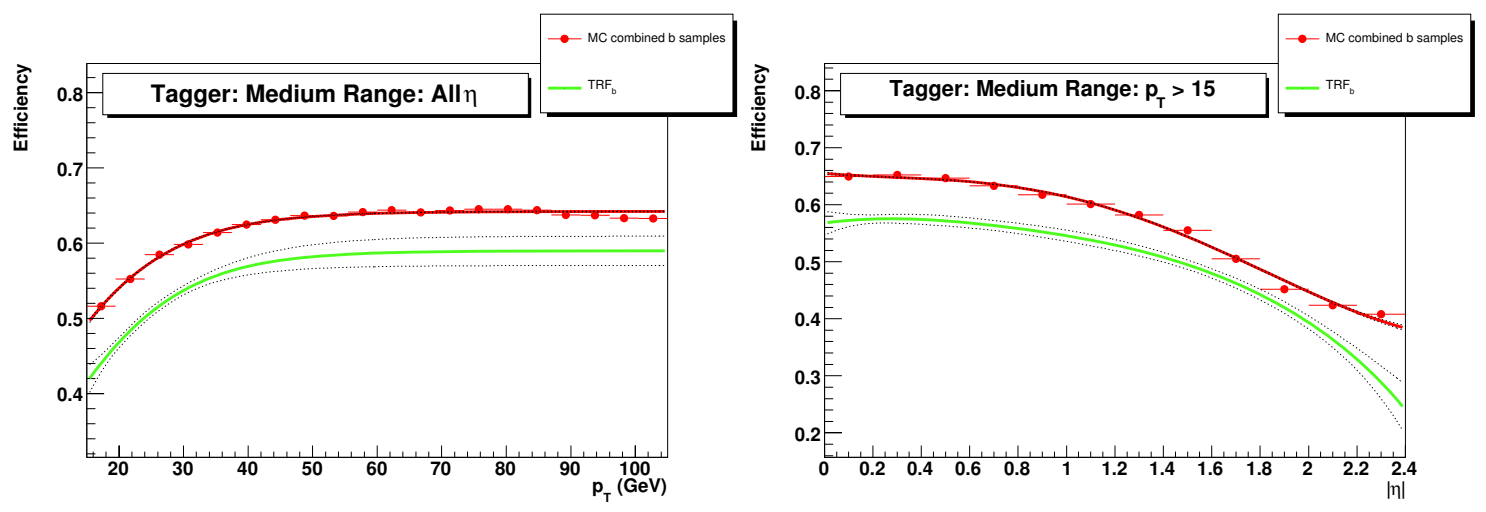

Figure 3.12: b-tagging efficiency in MC (red line) and TRF for b-jets (green line) with $1 \sigma$ error bands (black dashed line) on jet $p_{\mathrm{T}}$ (left) and $\eta$ (right) distributions [34]. 


\section{Chapter 4}

\section{Data Sample and Event Selection}

\subsection{Data Sample}

The data sample used in this analysis is collected from August 2002 to December 2005 by the $\mathrm{D} \emptyset$ detector. The run number range is $151,817-213,063$. The total integrated luminosity for the $e+$ jets channel is $912.5 \mathrm{pb}^{-1}$ and $871.3 \mathrm{pb}^{-1}$ for the $\mu+$ jets channel. The data taken during January and February in 2006 (about $\approx 60 \mathrm{pb}^{-1}$ ) is excluded in this analysis because cables for the calorimeter readout system were swapped. The data samples are processed using Common Analysis Format (CAF) developed by Common Sample Group (CSG) [46].

\begin{tabular}{|l||c|c|c|c|}
\hline \hline Trigger Version & Trigger Name & Delivered $\left(\mathrm{pb}^{-1}\right)$ & Recorded $\left(\mathrm{pb}^{-1}\right)$ & Good Quality $\left(\mathrm{pb}^{-1}\right)$ \\
\hline V8.0 - V9.0 & EM15_2JT15 & 6.17 & 4.94 & 4.86 \\
V9.0 - V10.0 & EM15_2JT15 & 47.84 & 41.73 & 24.73 \\
V10.0 - V11.0 & EM15_2JT15 & 20.35 & 18.29 & 9.81 \\
V11.0 - V12.0 & EM15_2JT15 & 78.87 & 71.83 & 62.82 \\
V12.0 - V13.0 & E1_SHT15_2J20 & 272.64 & 250.76 & 227.14 \\
V13.0 - V13.3 & E1_SHT15_2J_J25 & 80.43 & 73.02 & 54.81 \\
V13.3 - V14.0 & E1_SHT15_2J_J30 & 353.70 & 324.69 & 294.27 \\
V14.0 - V15.0 & E1_SHT15_2J_J25 & 290.01 & 270.62 & 234.11 \\
\hline Total & & 1150.01 & 1055.88 & 912.55 \\
\hline \hline
\end{tabular}

Table 4.1: Integrated luminosity for the triggers used in the $e+$ jets channel.

Table 4.1 and 4.2 show the integrated luminosities for the triggers used in both the $e+$ jets 


\begin{tabular}{|l||c|c|c|c|}
\hline \hline Trigger Version & Trigger Name & Delivered $\left(\mathrm{pb}^{-1}\right)$ & Recorded $\left(\mathrm{pb}^{-1}\right)$ & Good Quality $\left(\mathrm{pb}^{-1}\right)$ \\
\hline V8.0 - V9.0 & MU_JT20_L2M0 & 7.23 & 5.75 & 5.64 \\
V9.0 - V10.0 & MU_JT20_L2M0 & 47.89 & 41.77 & 24.77 \\
V10.0 - V11.0 & MU_JT20_L2M0 & 21.37 & 19.30 & 10.70 \\
V11.0 - V12.0 & MU_JT20_L2M0 & 79.27 & 74.34 & 65.24 \\
V12.0 - V13.0 & MU_JT25_L2M0 & 277.02 & 254.96 & 230.93 \\
V13.0 - V13.2 & MUJ2_JT25 & 55.74 & 39.47 & 31.43 \\
V13.2 - V13.3 & MUJ2_JT25_LM3 & 26.27 & 22.26 & 16.10 \\
V13.3 - V14.0 & MUJ2_JT30_LM3 & 382.01 & 277.12 & 252.17 \\
V14.0 - V14.2 & MUJ1_JT25_LM3 & 0.01 & 0.01 & 0.01 \\
V14.2 - V14.3 & MUJ1_JT25_ILM3 & 24.83 & 22.81 & 20.84 \\
V14.3 - V15.0 & MUJ1_JT35_LM3 & 265.45 & 248.06 & 213.51 \\
\hline Total & & 1187.09 & 1005.85 & 871.34 \\
\hline \hline
\end{tabular}

Table 4.2: Integrated luminosity for the triggers used in the $\mu+$ jets channel.

channel and the $\mu+$ jets channel. The triggers used in this analysis are described in Reference [47]. All integrated luminosities in the tables are derived using the new luminosity constant measured with the improved luminosity readout system $[48,49]$. The new constant includes effects from back propagation, radiation damage, and single diffractive efficiency corrections.

\subsection{Monte Carlo Samples}

In this analysis, the $t \bar{t} \mathrm{MC}$ sample is used to model the signal. Background processes such as $\mathrm{W}+\mathrm{jets}$, $\mathrm{Z}+$ jets, single top and diboson production, which can have the same final event signature as the $t \bar{t}$ lepton+jets channel, are also modeled using the MC. The PDF version used is CTEQ6L1 [40] for all of the MC samples.

The cross section measurement in this analysis is based on a $t \bar{t} \mathrm{MC}$ sample generated by PYTHIA 6.323 [41] with the top quark mass set to $175 \mathrm{GeV}$. The factorization scale is set to $m_{t}^{2}+\sum p_{T}^{2}$ (jets). Other $t \bar{t}$ MC samples with various top quark masses $(140,145,160,165,170,180,185,190,195$, $200,215 \mathrm{GeV}$ ) are also used to determine the mass dependence of the $t \bar{t}$ cross section. These MC samples are generated with same parameters as the main $175 \mathrm{GeV} t \bar{t}$ sample except for the top quark mass.

The main physics background, $\mathrm{W}+$ jets, is generated using ALPGEN MC event generator in- 
cluding a jet-matching algorithm prescribed by MLM [50]. The W+jets samples are split into three subsamples by parton flavors: $W b b, W c c$, and $W j j+$ extra light partons, where $b$ represents the bquark, $c$ represents the c-quark, and $j$ denotes a light-quark or gluon. The factorization scale for the samples is set to $m_{W}^{2}+p_{T}^{2}(W)$. The cross section for each subsample is provided by ALPGEN, but it does not include NLO corrections on the relative contributions for these subsamples. Therefore, a heavy flavor scale factor, the so-called K-factor, is used in this analysis. The measured K-factor is $1.17 \pm 0.18$ [51] and is applied to the number of expected events for both $W b b$ and $W c c$ samples. This K-factor is determined using data sample and $\mathrm{W}+$ jets $\mathrm{MC}$ sample with 2 jets multiplicity bin. The number of $\mathrm{W}+$ jets constribution is estimated using the b-tagged sample and untagged sample respectively, and then the K-factor is obtained by comparing the contributions determined in data and $\mathrm{W}+$ jets $\mathrm{MC}$ sample.

The other backgrounds, single top (s-channel and t-channel), $\mathrm{Z}(\rightarrow$ ee, $\mu \mu, \tau \tau, \nu \nu)+$ jets, and diboson (WW, WZ and ZZ) samples are also generated by MC simulation. CompHEP-singleTop [52] is used to simulate single top events with the top quark mass set to $175 \mathrm{GeV}$. The NLO cross section of $0.88(1.98) \mathrm{pb}$ is used for s-channel (t-channel) with $12.5 \%$ uncertainty [53]. Z+jets samples are generated by ALPGEN and also classified into 3 subsamples: $Z b b, Z c c$, and $Z j j$, the same as for the $\mathrm{W}+$ jets samples. To account for the NLO effect, a NLO factor of 1.23 is applied to all subsamples with $15 \%$ uncertainty and in addition a heavy flavor scale factor of 1.35 is applied to $Z b b$ and $Z c c$ subsamples with $50 \%$ uncertainty [54]. $\mathrm{Z} p_{\mathrm{T}}$ reweighting described in Reference [55] is applied to the $\mathrm{Z}+$ jets $\mathrm{MC}$ sample to correct the difference between the simulated $\mathrm{Z} p_{\mathrm{T}}$ distribtuion and data. This correction is measured using $Z \rightarrow e e$ samples for $\mathrm{MC}$ and data. Diboson samples are generated using PYTHIA with the following subchannels: $W W \rightarrow l+j e t s, W Z \rightarrow l+j e t s, W Z \rightarrow j j l \bar{l}, Z Z \rightarrow l \bar{l} j j$. The NLO cross section of 12.0 (WW), 3.68 (WZ), and 1.42 (ZZ) pb with $20 \%$ uncertainty are used for the diboson samples, respectively [56]. 


\subsection{Event Selection}

The purpose of event selection, so-called preselection, is to find an event candidate with the final event signature of the $t \bar{t}$ signal. This analysis is based on the lepton+jets channel of $t \bar{t}$, and therefore in the final state the event candidate should have one isolated high $p_{\mathrm{T}}$ lepton, large $E_{T}$ from a neutrino, and 4 or more jets. This event selection is designed to define a data sample enriched in $\mathrm{W}+$ jets and $t \bar{t}$ events. Using this event selection, the instrumental backgrounds like multijet events can be significantly removed.

The event selection criteria for both the $e+$ jets channel and the $\mu+$ jets channel are as follows. The same requirements are applied for data and $\mathrm{MC}$ samples. Common requirements for both channels are:

- Good quality [57] (for data)

- Exactly three or four or more jets with $p_{T}>20 \mathrm{GeV}$ and $|\eta|<2.5$

- Leading jet $p_{T}>40 \mathrm{GeV}$

- Good vertex with $\left|z_{P V}\right| \leq 60 \mathrm{~cm}$ and at least 3 tracks attached to the vertex.

For the $e+$ jets channel only:

- One tight electron with $p_{T}>20 \mathrm{GeV}$ in the $\mathrm{CC}$

- No second tight electron with $p_{T}>15 \mathrm{GeV}$ in the $\mathrm{CC}$ or EC

- No isolated muon with $p_{T}>15 \mathrm{GeV}$

- Electron coming from the primary vertex: $|\Delta z(e, P V)|<1 \mathrm{~cm}$

- $E_{T}>20 \mathrm{GeV}$ and triangle cut $\Delta \phi\left(e, E_{T}\right)>0.7 \cdot \pi-0.045 \cdot E_{T}$,

where tight electron denotes the electron candidate passed the requirements described in Section 3.1.3. To make this analysis orthogonal to the analysis using dilepton channel, events with a second electron with high $p_{\mathrm{T}}$ are explicitly vetoed.

For the $\mu+$ jets channel only: 
- One tight muon with $p_{T}>20 \mathrm{GeV}$

- Invariant mass of the selected muon and any second muon $m_{\mu \mu}<70 \mathrm{GeV}$ or $m_{\mu \mu}>110 \mathrm{GeV}$ to reject $Z(\rightarrow \mu \mu)+$ jets events

- No second muon with $p_{T}>15 \mathrm{GeV}$ with muon quality Medium and $n s e g=3$

- No tight electron with $p_{T}>15 \mathrm{GeV}$

- Muon coming from the primary vertex: $|\Delta z(\mu, P V)|<1 \mathrm{~cm}$

- $E_{T}>25 \mathrm{GeV}$ and triangle cut $\Delta \phi\left(\mu, E_{T}\right)>2.1-0.035 \cdot E_{T}$,

where tight muon denotes the muon candidates passed the requirements described in Section 3.1.4.

Figures 4.1 and 4.2 show the distributions for leading jet $p_{\mathrm{T}}$ (left) and $E_{T}$ (right) in the e+jets channel and the $\mu+$ jets channel. In the figures, the black solid line is the distribution for the $t \bar{t}$ signal events (MC), the red dotted line is the distribution for the multijet background events (data), and the blue dot-dashed line is the leading jet $p_{\mathrm{T}}$ cut (left) and the $E_{T}$ cut (right) used in this analysis.
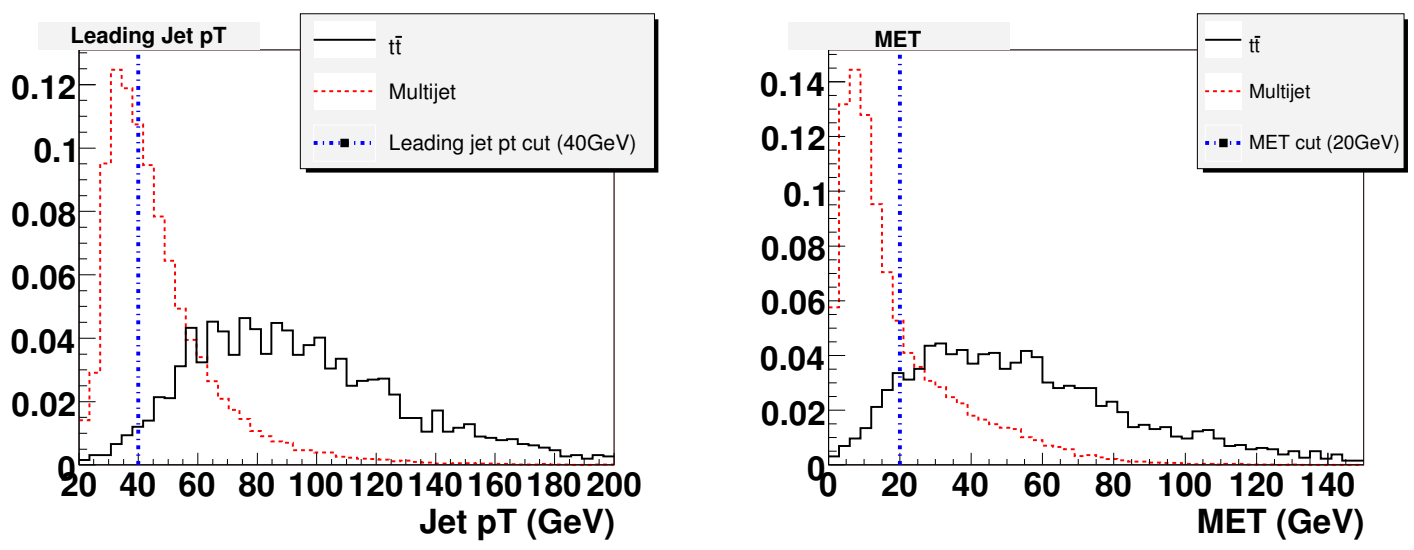

Figure 4.1: Leading jet $p_{\mathrm{T}}$ (left) and $E_{T}$ distributions for $t \bar{t}$ signal events (MC) and multijet background events (data) in the $e+$ jets channel. The black solid line is for $t \bar{t}$ events and the red dotted line is for multijet events. The blue dot-dashed line is the leading jet $p_{\mathrm{T}}$ cut (left) and the $\mathbb{E}_{T}$ cut (right) used in this analysis. The y-axis is normalized to 1. 

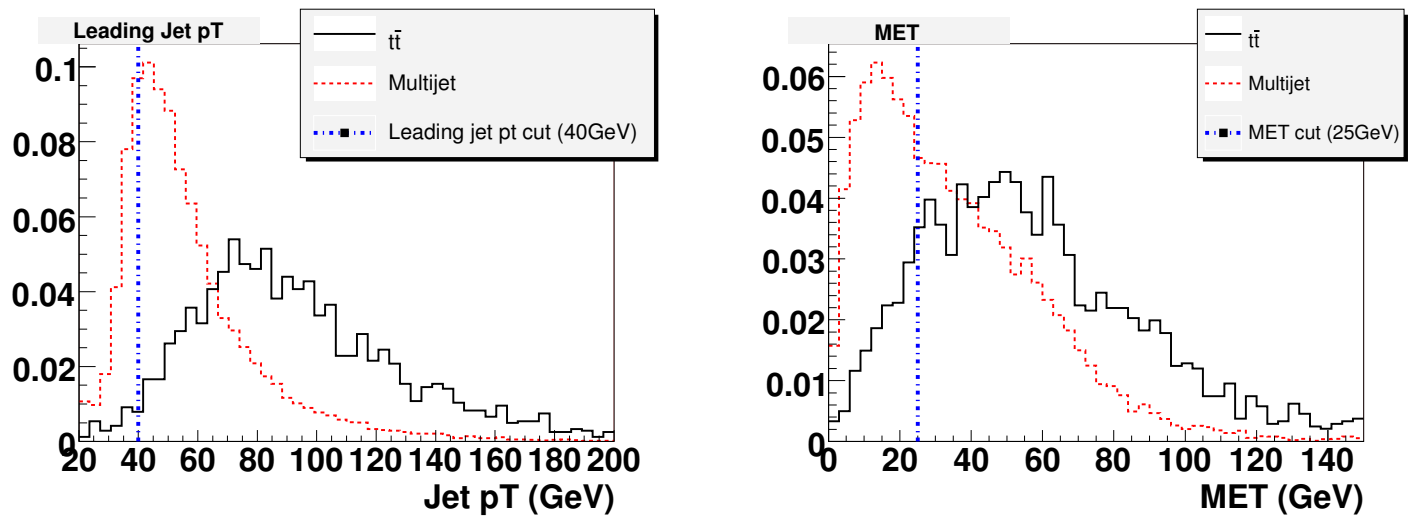

Figure 4.2: Leading jet $p_{\mathrm{T}}$ (left) and $\mathbb{E}_{T}$ distributions for $t \bar{t}$ signal events (MC) and multijet background events (data) in the $\mu+$ jets channel. The black solid line is for $t \bar{t}$ events and the red dotted line is for multijet events. The blue dot-dashed line is the leading jet $p_{\mathrm{T}}$ cut (left) and the $E_{T}$ cut (right) used in this analysis. The y-axis is normalized to 1.

\subsubsection{Triangle Cut}

The instrumental background in this analysis is multijet events. The multijet events can have the same final state as the signal by having a jet misidentifed as a lepton or a lepton produced inside a jet. To remove the background without suffering from a large selection efficiency loss, so-called "triangle cut" is applied.

Figure 4.3 (4.4) shows the distribution of $\Delta \phi\left(e, E_{T}\right)\left(\Delta \phi\left(\mu, E_{T}\right)\right)$ versus $E_{T}$ plane for QCD enriched multijet events obtained by inverting the electron (muon) isolation cuts in the $e+$ jets $(\mu+$ jets $)$ channel. $\mathbb{E}_{T}$ can result from inaccurately measuring jet energies due to the finite jet energy resolution. By this mismeasurement, events tend to accumulate in the low $\Delta \phi\left(\ell, E_{T}\right)$ region and are shifted toward higher $E_{T}$ values as shown in Figure 4.3 and 4.4. Therefore, multijet events can be more effectively suppressed by applying cuts on both $E_{T}$ and $\Delta \phi\left(\ell, E_{T}\right)$ versus $E_{T}$ than only on $E_{T}$. The black solid lines denote the $E_{T}$ cut and the triangle cut in the figures.

\subsubsection{Event Selection Efficiency for $t \bar{t}$ MC Sample}

Event selection efficiency for the $t \bar{t}$ signal is determined using $t \bar{t}$ MC events with $t \bar{t} \rightarrow \ell \nu_{\ell} q \bar{q}^{\prime} b \bar{b}$. The cut flow, number of events after cuts, exclusive efficiency for each cut, and cumulative efficiencies are shown in Table 4.3 for the 3 jet multiplicity bin and Table 4.4 for the 4 or more jet multiplicity bin in 

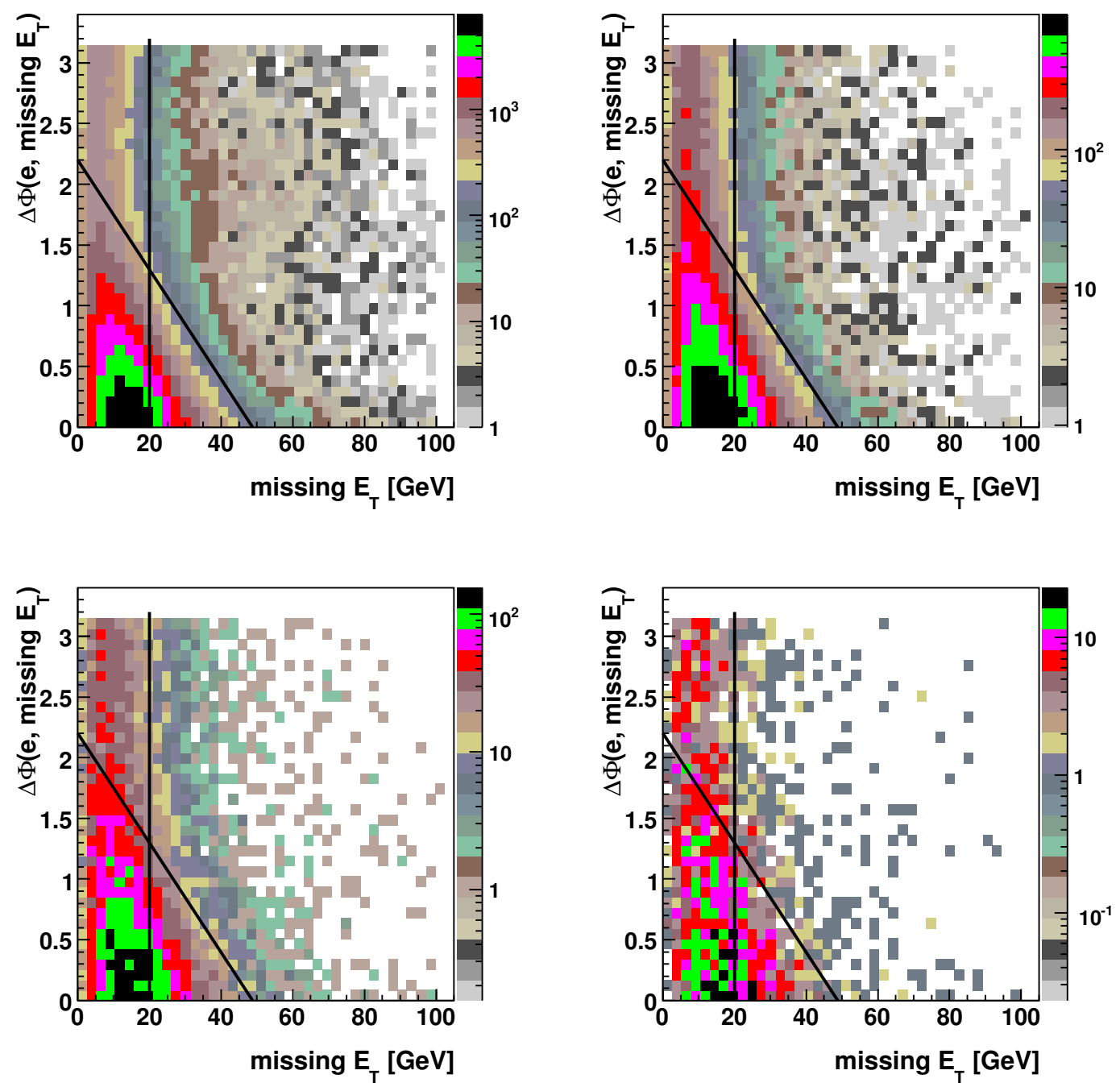

Figure 4.3: $\Delta \phi\left(e, \mathbb{E}_{T}\right)$ vs. $\mathbb{E}_{T}$ distributions for multijet events in the $e+$ jets channel. Jet multiplicities in the $1,2,3, \geq 4$ bins are shown as follows 1 jet (top left), 2 jets (top right), 3 jets (bottom left), and $\geq 4$ jets (bottom right) [58]. 

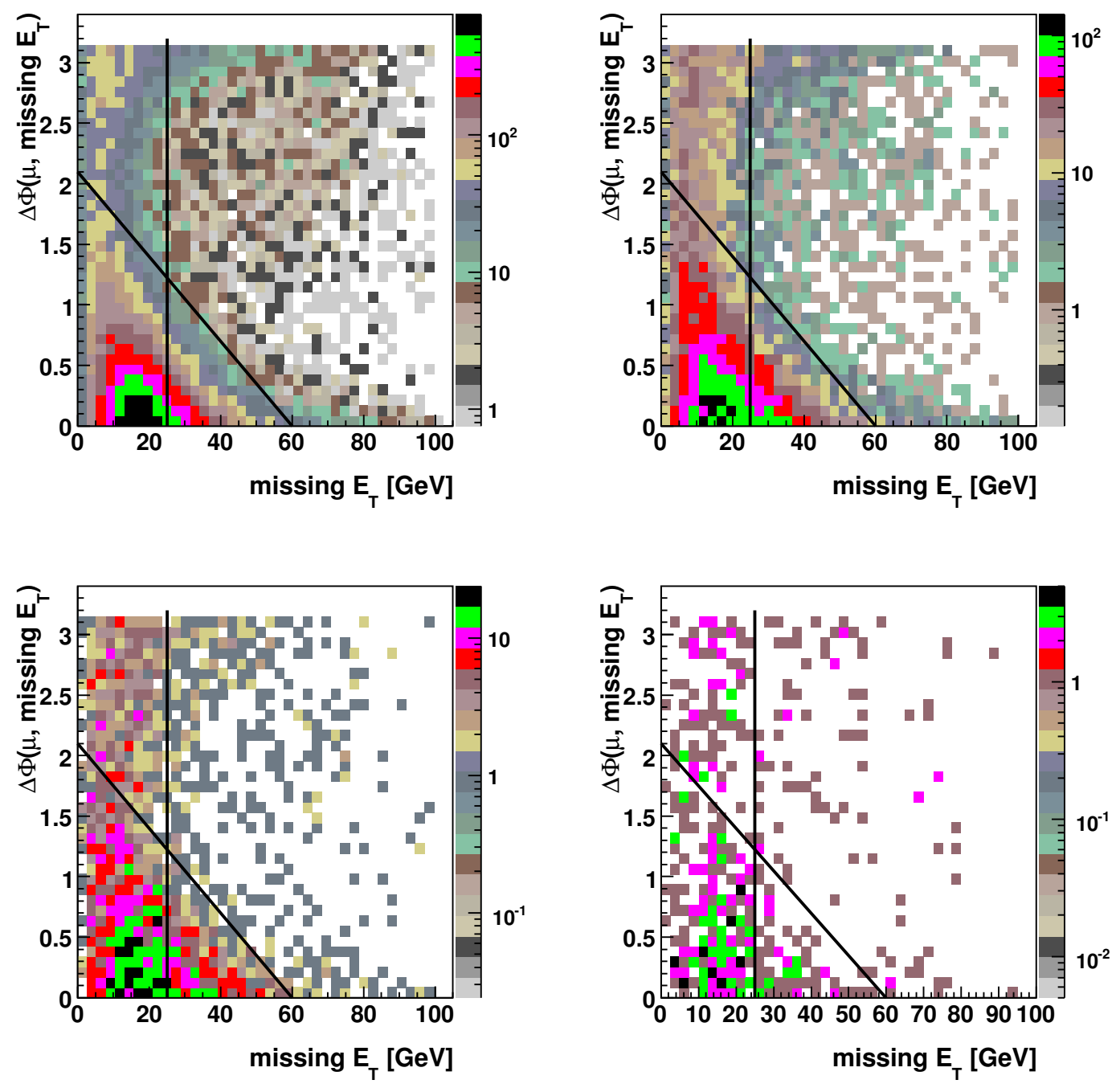

Figure 4.4: $\Delta \phi\left(\mu, E_{T}\right)$ vs. $E_{T}$ distributions for multijet events in the $\mu+$ jets channel. Jet multiplicities in the 1,2,3, $\geq 4$ bins are shown as follows 1 jet (top left), 2 jets (top right), 3 jets (bottom left), and $\geq 4$ jets (bottom right) [58]. 
the $e+$ jets channel. Table 4.5 shows the same quantities for the 3 jet multiplicity bin and Table 4.6 for the 4 or more jet multiplicity bin in the $\mu+$ jets channel. Some of the correction factors are multiplied to calculate the efficiencies. The MC-to-data correction factors are typically derived using a control sample that allows the efficiency to be extracted on both MC and collider data. Selection efficiency for the data quality requirement is also considered in calculating the event selection efficiency. This efficiency is $97.14 \%$ and is measured with an insignificant statistical error [59].

The contribution from the $t \bar{t}$ dilepton channel is not negligible, in spite of the second lepton veto cut. Therefore, the number of events from the dilepton channel after event selection are used in the calculation of the cross section. Table 4.7 shows the event selection efficiency for $t \bar{t}$ dilepton contribution.

\begin{tabular}{|l|r|r|r|}
\hline \hline Selection or $\kappa$ & Events left & Exclusive efficiency[\%] & Cumulative efficiency[\%] \\
\hline $\mathrm{N}_{\text {jets }}=3$ & 242352 & & \\
Leading jet $>40 \mathrm{GeV}$ & 92381 & $38.12 \pm 0.10$ & $38.12 \pm 0.10$ \\
Loose electron & 91156 & $98.67 \pm 0.04$ & $37.61 \pm 0.10$ \\
Muon veto & 48338 & $53.03 \pm 0.17$ & $19.95 \pm 0.08$ \\
2nd electron veto & 48329 & $99.98 \pm 0.01$ & $19.94 \pm 0.08$ \\
Vertex selection & 48318 & $99.98 \pm 0.01$ & $19.94 \pm 0.08$ \\
$E_{T}>20 \mathrm{GeV}$ & 42301 & $98.58 \pm 0.05$ & $19.65 \pm 0.08$ \\
Triangle selection & 39735 & $88.81 \pm 0.14$ & $17.45 \pm 0.08$ \\
Tight electron & 35111 & $93.93 \pm 0.12$ & $16.40 \pm 0.08$ \\
\hline Trigger probability & & $88.36 \pm 0.16$ & $14.49 \pm 0.07$ \\
$\kappa_{\text {electron reco, ID }}$ & & $0.966 \pm 0.000$ & $14.00 \pm 0.07$ \\
$\kappa_{\text {electron likelihood }}$ & & $0.985 \pm 0.000$ & $13.79 \pm 0.07$ \\
\hline Data Quality & & $0.891 \pm 0.000$ & $12.28 \pm 0.06$ \\
\hline$\varepsilon^{\text {total }}$ & & $0.971 \pm 0.005$ & $11.93 \pm 0.09$ \\
\hline \hline
\end{tabular}

Table 4.3: Summary of the $t \bar{t} \rightarrow e+$ jets event preselection efficiencies and the corresponding scale factors $(\kappa)$, as applicable, for exactly three jets. Only statistical uncertainties are included.

\section{4 b-tagging Optimization}

There are totally 12 operating points for the NN tagger [34]. The NN cuts for each operating point are shown in Table 4.8. The optimized NN operating point is selected using the $S / \sqrt{S+B}$ ratio. $S$ indicates the number of signal events and $B$ is the number of background events. The 


\begin{tabular}{|l|r|r|r|}
\hline \hline Selection or $\kappa$ & Events left & Exclusive efficiency[\%] & Cumulative efficiency[\%] \\
\hline $\mathrm{N}_{\text {jets }} \geq 4$ & 242352 & & \\
Leading jet $>40 \mathrm{GeV}$ & 115770 & $47.77 \pm 0.10$ & $47.77 \pm 0.10$ \\
Loose electron & 113856 & $98.35 \pm 0.04$ & $46.98 \pm 0.10$ \\
Muon veto & 47122 & $41.39 \pm 0.15$ & $19.44 \pm 0.08$ \\
2nd electron veto & 47105 & $99.98 \pm 0.01$ & $19.44 \pm 0.08$ \\
Vertex selection & 46378 & $99.98 \pm 0.01$ & $19.44 \pm 0.08$ \\
$E_{T}>20$ GeV & 40998 & $98.46 \pm 0.06$ & $19.14 \pm 0.08$ \\
Triangle selection & 38557 & $98.40 \pm 0.15$ & $16.92 \pm 0.08$ \\
Tight electron & 34565 & $89.65 \pm 0.12$ & $15.91 \pm 0.07$ \\
\hline Trigger probability & & $0.968 \pm 0.000$ & $14.26 \pm 0.07$ \\
$\kappa_{\text {electron reco, ID }}$ & & $0.985 \pm 0.000$ & $13.81 \pm 0.07$ \\
$\kappa_{\text {electron likelihood }}$ & & $0.891 \pm 0.000$ & $13.60 \pm 0.07$ \\
\hline Data Quality & $0.971 \pm 0.005$ & $12.12 \pm 0.06$ \\
\hline$\varepsilon^{\text {total }}$ & & $11.77 \pm 0.09$ \\
\hline \hline
\end{tabular}

Table 4.4: Summary of the $t \bar{t} \rightarrow e+j e t s$ event preselection efficiencies and the corresponding scale factors $(\kappa)$, as applicable, for four or more jets. Only statistical uncertainties are included.

\begin{tabular}{|c|c|c|c|}
\hline Selection or $\kappa$ & Events left & Exclusive efficiency[\%] & Cumulative efficiency[\%] \\
\hline & 240919 & & \\
\hline $\mathrm{N}_{\mathrm{jets}}=3$ & 94436 & $39.20 \pm 0.10$ & $39.20 \pm 0.10$ \\
\hline Leading jet $>40 \mathrm{GeV}$ & 93400 & $98.90 \pm 0.03$ & $38.77 \pm 0.10$ \\
\hline Loose muon & 42345 & $45.34 \pm 0.16$ & $17.58 \pm 0.08$ \\
\hline 2nd muon veto & 42339 & $99.99 \pm 0.01$ & $17.57 \pm 0.08$ \\
\hline Electron veto & 42299 & $99.91 \pm 0.01$ & $17.56 \pm 0.08$ \\
\hline Vertex selection & 41706 & $98.60 \pm 0.06$ & $17.31 \pm 0.08$ \\
\hline$E_{T}>25 \mathrm{GeV}$ & 35809 & $85.86 \pm 0.17$ & $14.86 \pm 0.07$ \\
\hline Triangle selection & 33313 & $93.03 \pm 0.13$ & $13.83 \pm 0.07$ \\
\hline Tight muon & 29133 & $87.45 \pm 0.18$ & $12.09 \pm 0.07$ \\
\hline Trigger probability & & $0.877 \pm 0.001$ & $10.61 \pm 0.06$ \\
\hline$\kappa_{\mu I D \times a c c \times}$ cosmic veto & & $0.956 \pm 0.001$ & $10.14 \pm 0.06$ \\
\hline$\kappa_{\mu \text { track }}$ & & $0.855 \pm 0.001$ & $8.67 \pm 0.05$ \\
\hline$\kappa_{\mu+\text { jets iso corr }}$ & & $1.102 \pm 0.000$ & $9.55 \pm 0.06$ \\
\hline$\kappa_{\text {Rat } 11<0.08 \text { and Rattrk }<0.06}$ & & $1.002 \pm 0.000$ & $9.57 \pm 0.06$ \\
\hline Data Quality & & $0.971 \pm 0.005$ & $9.30 \pm 0.07$ \\
\hline$\varepsilon^{\text {total }}$ & & & $9.30 \pm 0.07$ \\
\hline
\end{tabular}

Table 4.5: Summary of the $t \bar{t} \rightarrow \mu+$ jets event preselection efficiencies and the corresponding scale factors $(\kappa)$, as applicable, for exactly three jets. Only statistical uncertainties are included. 


\begin{tabular}{|l|r|r|r|}
\hline \hline Selection or $\kappa$ & Events left & Exclusive efficiency[\%] & Cumulative efficiency[\%] \\
\hline $\mathrm{N}_{\text {jets }} \geq 4$ & 240919 & & \\
Leading jet $>40 \mathrm{GeV}$ & 116286 & $48.27 \pm 0.10$ & $48.27 \pm 0.10$ \\
Loose muon & 115253 & $99.11 \pm 0.03$ & $47.84 \pm 0.10$ \\
2nd muon veto & 47432 & $41.15 \pm 0.14$ & $19.69 \pm 0.08$ \\
Electron veto & 47431 & $100.00 \pm 0.00$ & $19.69 \pm 0.08$ \\
Vertex selection & 47393 & $99.92 \pm 0.01$ & $19.67 \pm 0.08$ \\
$E_{T}>25$ GeV & 46716 & $98.57 \pm 0.05$ & $19.39 \pm 0.08$ \\
Triangle selection & 39740 & $85.07 \pm 0.16$ & $16.50 \pm 0.08$ \\
Tight muon & 36982 & $93.06 \pm 0.13$ & $15.35 \pm 0.07$ \\
\hline Trigger probability & 32027 & $86.60 \pm 0.18$ & $13.29 \pm 0.07$ \\
$\kappa_{\mu}$ ID $\times$ acc $\times$ cosmic veto & & $0.878 \pm 0.001$ & $11.67 \pm 0.06$ \\
$\kappa_{\mu \text { track }}$ & & $0.957 \pm 0.001$ & $11.17 \pm 0.06$ \\
$\kappa_{\mu+\text { jets iso corr }}$ & $0.854 \pm 0.001$ & $9.54 \pm 0.05$ \\
$\kappa_{\text {Rat11 }<0.08 \text { and Rattrk }<0.06}$ & & $1.003 \pm 0.000$ & $9.57 \pm 0.05$ \\
\hline Data Quality & & $1.002 \pm 0.000$ & $9.59 \pm 0.05$ \\
\hline$\varepsilon^{\text {total }}$ & & $9.971 \pm 0.005$ & $9.31 \pm 0.07$ \\
\hline \hline
\end{tabular}

Table 4.6: Summary of the $t \bar{t} \rightarrow \mu+$ jets event preselection efficiencies and the corresponding scale factors $(\kappa)$, as applicable, for four or more jets. Only statistical uncertainties are included.

\begin{tabular}{|l|c|c|}
\hline \hline$N_{\text {Jets }}$ & $e+j e t s$ & $\mu+j e t s$ \\
\hline$=3$ jets $(\%)$ & $7.66 \pm 0.10$ & $1.64 \pm 0.05$ \\
$\geq 4$ jets (\%) & $6.40 \pm 0.09$ & $1.37 \pm 0.04$ \\
\hline
\end{tabular}

Table 4.7: Preselection efficiency for $t \bar{t}$ dilepton MC samples. The error is statistical only.

$S / \sqrt{S+B}$ ratio is measured for all of operating points and then the best ratio is selected. $t \bar{t}$ MC samples are used as signal events and other MC samples ( $\mathrm{W}+$ jets, $\mathrm{Z}+$ jets, and single top) are used as backgrounds to calculate the $S / \sqrt{S+B}$ ratio. Table 4.9 and 4.10 show the results for the $S / \sqrt{S+B}$ ratio of the individual operating points. In this analysis, the "MEDIUM" operating point is chosen for the NN tagger.

\begin{tabular}{|l|c|c|c|c|c|c|}
\hline \hline Name & MegaTight & UltraTight & VeryTight & Tight & Medium & oldLoose \\
\hline NN cut & $>0.925$ & $>0.9$ & $>0.85$ & $>0.775$ & $>0.65$ & $>0.5$ \\
\hline \hline Name & Loose & L2 & L3 & L4 & L5 & L6 \\
\hline NN cut & $>0.45$ & $>0.325$ & $>0.25$ & $>0.2$ & $>0.15$ & $>0.1$ \\
\hline \hline
\end{tabular}

Table 4.8: The NN tagger's operating points [34]. 


\begin{tabular}{|c|c|c|c|}
\hline \hline e+jets 3 exclusive & $S$ & $B$ & $S / \sqrt{S+B}$ \\
\hline MegaTight & 67.3 & 77.9 & 5.6 \\
Tight & 78.6 & 107.3 & 5.8 \\
Medium & 84.7 & 130.6 & 5.8 \\
Loose & 92.5 & 177.1 & 5.6 \\
L2 & 97.2 & 223.3 & 5.4 \\
L3 & 100.9 & 269.7 & 5.2 \\
L4 & 103.6 & 314.9 & 5.1 \\
L6 & 109.0 & 441.7 & 4.6 \\
\hline e+jets 4 inclusive & $S$ & $B$ & $S / \sqrt{S+B}$ \\
\hline MegaTight & 68.3 & 15.0 & 7.5 \\
Tight & 79.2 & 20.6 & 7.9 \\
Medium & 85.0 & 25.2 & 8.1 \\
Loose & 92.1 & 34.8 & 8.2 \\
L2 & 96.3 & 44.2 & 8.1 \\
L3 & 99.5 & 52.9 & 8.1 \\
L4 & 101.8 & 63.4 & 7.9 \\
L6 & 106.2 & 87.2 & 7.6 \\
\hline MegaTight & 135.6 & 92.9 & $S / \sqrt{S+B}$ \\
Tight & 157.9 & 127.9 & 9.0 \\
Medium & 169.7 & 155.8 & 9.4 \\
Loose & 184.6 & 211.9 & 9.3 \\
L2 & 193.5 & 267.5 & 9.0 \\
L3 & 200.3 & 322.6 & 8.8 \\
L4 & 205.4 & 378.3 & 8.5 \\
L6 & 215.2 & 528.9 & 7.9 \\
\hline \hline
\end{tabular}

Table 4.9: Signal and background ratios of different operating points in the $e+$ jets channel. The best operating point has been chosen to maximize $S / \sqrt{S+B}$ for the third inclusive jet bin. 


\begin{tabular}{|c|c|c|c|}
\hline \hline$\mu+$ jets 3 exclusive & $S$ & $B$ & $S / \sqrt{S+B}$ \\
\hline MegaTight & 57.0 & 74.6 & 5.0 \\
Tight & 66.4 & 102.4 & 5.1 \\
Medium & 71.4 & 124.8 & 5.1 \\
Loose & 77.8 & 168.0 & 5.0 \\
L2 & 81.6 & 208.0 & 4.8 \\
L3 & 84.5 & 248.3 & 4.6 \\
L4 & 86.7 & 286.6 & 4.5 \\
L6 & 91.0 & 401.7 & 4.1 \\
\hline$\mu+$ jets 4 inclusive & $S$ & $B$ & $S / \sqrt{S+B}$ \\
\hline MegaTight & 65.6 & 13.9 & 7.3 \\
Tight & 74.6 & 21.0 & 7.6 \\
Medium & 79.9 & 26.0 & 7.7 \\
Loose & 86.4 & 35.7 & 7.8 \\
L2 & 90.2 & 46.2 & 7.7 \\
L3 & 93.0 & 56.6 & 7.6 \\
L4 & 95.1 & 68.0 & 7.5 \\
L6 & 99.0 & 96.1 & 7.1 \\
\hline Mets 3 inclusive & $S$ & $B$ & $S / \sqrt{S+B}$ \\
\hline MegaTight & 121.5 & 88.5 & 8.4 \\
Tight & 141.0 & 123.4 & 8.7 \\
Medium & 151.3 & 150.8 & 8.7 \\
Loose & 164.2 & 203.7 & 8.6 \\
L2 & 171.8 & 254.2 & 8.3 \\
L3 & 177.6 & 304.9 & 8.1 \\
L4 & 181.8 & 354.6 & 7.9 \\
L6 & 190.0 & 497.8 & 7.2 \\
\hline \hline
\end{tabular}

Table 4.10: Signal and background ratios of different operating points in the $\mu+$ jets channel. The best operating point has been chosen to maximize $S / \sqrt{S+B}$ for the third inclusive jet bin. 


\section{Chapter 5}

\section{Background Estimation}

This chapter describes the procedure used to estimate the background that passes the event selection discussed in Section 4.3. The background can be classified into two different types: instrumental background and physics background. The instrumental background is mainly multijet events with four or more jets which can be produced by the strong interaction. Such events can pass the event selection by having one or more of the following: $\mathbb{E}_{T}$ mismeasurement, a fake tight electron produced inside a jet, a photon associated with a random track, a fake muon from hadron punchthrough, a lepton produced by the leptonic decay of a heavy flavor jet where a heavy flavor jet is not reconstruncted. Physics backgrounds are from events that have the same final state as the $t \bar{t}$ signal, such as radiative $\mathrm{W}$ production accompanied by four or more jets. Other physics backgrounds with small contributions are single top, $\mathrm{Z}+$ jets and diboson events.

\subsection{Instrumental Backgrounds}

The "matrix method" is used to estimate not only the contributions of a fake lepton originating from multijet events but also the contribution of real isolated leptons from $t \bar{t}$ signal or physics backgrounds in the data sample passing the event selection. In the matrix method, two subsamples are defined for each jet multiplicity $(n=1,2,3, \geq 4)$ : the tight sample and the loose sample. The tight sample is the collection of events passing all criteria of the event selection that are described in Section 4.3 
for the tight lepton selection. The loose sample is the same as the tight sample except that the loose lepton selection (without the likelihood cut for electrons or isolation cut for muons) is applied. Therefore, the tight sample is a subset of the loose sample. The formula for matrix method is

$$
\begin{aligned}
N_{\ell} & =N_{\ell}^{\mathrm{W}+\mathrm{t} \overline{\mathrm{t}}}+N_{\ell}^{\mathrm{QCD}} \\
N_{t} & =N_{t}^{\mathrm{W}+\mathrm{t} \overline{\mathrm{t}}}+N_{t}^{\mathrm{QCD}} \\
& =\varepsilon_{s i g} N_{\ell}^{\mathrm{W}+\mathrm{t} \overline{\mathrm{t}}}+\varepsilon_{Q C D} N_{\ell}^{\mathrm{QCD}}
\end{aligned}
$$

where $N_{t}$ and $N_{\ell}$ denote the number of events for the tight sample and the loose sample, respectively. $N_{\ell}^{\mathrm{W}+\mathrm{t} \overline{\mathrm{t}}}\left(N_{t}^{\mathrm{W}+\mathrm{t} \overline{\mathrm{t}}}\right)$ is the total number of physics background and $t \bar{t}$ signal events in the loose (tight) sample, and $N_{\ell}^{\mathrm{QCD}}\left(N_{t}^{\mathrm{QCD}}\right)$ is the contribution of multijet events in the loose (tight) sample. $\varepsilon_{s i g}$ is the efficiency for a real lepton in the loose sample to pass the tight lepton selection and $\varepsilon_{Q C D}$ is the efficiency for a fake lepton in the loose sample to pass the tight lepton selection. This system of two linear equations can be solved for $N_{\ell}^{\mathrm{QCD}}$ and $N_{\ell}^{\mathrm{W}+\mathrm{t} \overline{\mathrm{t}}}$

$$
N_{\ell}^{\mathrm{W}+\mathrm{t} \overline{\mathrm{t}}}=\frac{N_{t}-\varepsilon_{Q C D} N_{\ell}}{\varepsilon_{s i g}-\varepsilon_{Q C D}} \quad \text { and } \quad N_{\ell}^{\mathrm{QCD}}=\frac{\varepsilon_{s i g} N_{\ell}-N_{t}}{\varepsilon_{s i g}-\varepsilon_{Q C D}} .
$$

Therefore, $N_{\ell}^{\mathrm{W}+\mathrm{t} \overline{\mathrm{t}}}$ and $N_{\ell}^{\mathrm{QCD}}$ can be determined if $\varepsilon_{\text {sig }}$ and $\varepsilon_{Q C D}$ are measured.

$\varepsilon_{s i g}$ can be obtained from the corresponding $W+$ jets and $t \bar{t} \mathrm{MC}$ samples by measuring the fraction of events passing the loose selection that also pass the tight selection. A correction factor for the differences between $\mathrm{MC}$ and data is applied. $\varepsilon_{Q C D}$ is obtained from the low $\mathbb{E}_{T}$ region where the multijet events are dominant. $\varepsilon_{Q C D}$ is measured directly from the data sample by measuring the ratio of the number of events in the tight sample to the loose sample with $\mathscr{E}_{T}<10 \mathrm{GeV}$ [58]. Table 5.1 shows the measured $\varepsilon_{s i g}[60]$ and $\varepsilon_{Q C D}[58]$ for each jet multiplicity for both the $e+$ jets and $\mu+$ jets channels. In this table, the systematic uncertainty is obtained by measuring the dependence of various kinematic variables such as lepton $p_{\mathrm{T}}$, lepton $\eta$, lepton $\phi$, leading jet $\eta$, and the number of primary vertices. The biggest deviation of their dependences from the central value is chosen as the systematice uncertainty. 


\begin{tabular}{|l|c|c|c|c|}
\hline \hline \multirow{2}{*}{$N_{\text {jets }}$} & \multicolumn{2}{|c|}{$e+j e t s$} & \multicolumn{2}{c|}{$\mu+j e t s$} \\
\cline { 2 - 5 } & $\varepsilon_{\text {sig }}(\%)$ & $\varepsilon_{Q C D}(\%)$ & $\varepsilon_{\text {sig }}(\%)$ & $\varepsilon_{Q C D}(\%)$ \\
\hline$=1$ jet & $83.6 \pm 0.5 \pm 3.5$ & & $91.5 \pm 0.6 \pm 0.9$ & \\
$=2$ jets & $84.6 \pm 0.3 \pm 1.5$ & $19.5 \pm 0.4 \pm 1.7$ & $88.7 \pm 0.3 \pm 0.7$ & $27.2 \pm 1.3 \pm 5.2$ \\
$=3$ jets & $84.8 \pm 0.3 \pm 0.1$ & & $87.3 \pm 0.3 \pm 0.5$ & \\
$\geq 4$ jets & $84.0 \pm 0.4 \pm 1.8$ & & $84.5 \pm 0.4 \pm 2.2$ & \\
\hline \hline
\end{tabular}

Table 5.1: $\varepsilon_{s i g}$ for different jet multiplicities in both channels. $\varepsilon_{Q C D}$ is measured without a jet multiplicity dependence. The first error is the statistical error and the second one is the systematic error (\%).

Using the measured $\varepsilon_{s i g}$ and $\varepsilon_{Q C D}$, the contributions of $N_{t}^{\mathrm{W}+\mathrm{t} \overline{\mathrm{t}}}$ and $N_{t}^{\mathrm{QCD}}$ in the preselected events of the tight sample are calculated using Equation 5.3.

$$
N_{t}^{\mathrm{W}+\mathrm{t} \overline{\mathrm{t}}}=\varepsilon_{s i g} \frac{N_{t}-\varepsilon_{Q C D} N_{\ell}}{\varepsilon_{s i g}-\varepsilon_{Q C D}} \quad \text { and } \quad N_{t}^{\mathrm{QCD}}=\varepsilon_{Q C D} \frac{\varepsilon_{\text {sig }} N_{\ell}-N_{t}}{\varepsilon_{s i g}-\varepsilon_{Q C D}} .
$$

Tables 5.2 and 5.3 show the values of $N_{t}^{\mathrm{W}+\mathrm{tt}}$ and $N_{t}^{\mathrm{QCD}}$ determined by matrix method in the $e+$ jets and $\mu+$ jets channels, respectively. In the tables, "before b-tagging" row shows the results in the preselected sample.

The multijet background contribution in the b-tagged sample is determined by the matrix method using the same procedure that is described above. The matrix method is applied to the b-tagged sample directly. Equations 5.4, 5.5, and 5.6 show the formula and solutions

$$
\begin{aligned}
& N_{\ell}^{\text {tag }}=N_{\ell}^{\text {tag, } \mathrm{W}+\mathrm{t} \overline{\mathrm{t}}}+N_{\ell}^{\text {tag, } \mathrm{QCD}} \\
& N_{t}^{\text {tag }}=\quad N_{t}^{\text {tag, }, \mathrm{W}+\mathrm{t} \overline{\mathrm{t}}}+\quad N_{t}^{\text {tag, }, \mathrm{QCD}} \\
& =\varepsilon_{s i g} N_{\ell}^{\text {tag, } \mathrm{W}+\mathrm{t} \overline{\mathrm{t}}}+\varepsilon_{Q C D} N_{\ell}^{\text {tag }, \mathrm{QCD}} \\
& N_{\ell}^{t a g, \mathrm{~W}+\mathrm{t} \overline{\mathrm{t}}}=\frac{N_{t}^{t a g}-\varepsilon_{Q C D} N_{\ell}^{t a g}}{\varepsilon_{s i g}-\varepsilon_{Q C D}} \quad \text { and } \quad N_{\ell}^{t a g, \mathrm{QCD}}=\frac{\varepsilon_{s i g} N_{\ell}^{t a g}-N_{t}^{t a g}}{\varepsilon_{s i g}-\varepsilon_{Q C D}} \\
& N_{t}^{\text {tag }, \mathrm{W}+\mathrm{t} \overline{\mathrm{t}}}=\varepsilon_{s i g} \frac{N_{t}^{t a g}-\varepsilon_{Q C D} N_{\ell}^{t a g}}{\varepsilon_{s i g}-\varepsilon_{Q C D}} \quad \text { and } \quad N_{t}^{\text {tag, } \mathrm{QCD}}=\varepsilon_{Q C D} \frac{\varepsilon_{s i g} N_{\ell}^{\text {tag }}-N_{t}^{\text {tag }}}{\varepsilon_{s i g}-\varepsilon_{Q C D}}
\end{aligned}
$$


where "tag" indicates the b-tagged samples. The values of $\varepsilon_{s i g}$ and $\varepsilon_{Q C D}$ are given in Table 5.1 since they are not expected to have a dependence on the flavor composition of the preselected events. In the Tables 5.2 and 5.3, "exactly one b-tag" means that an event is found with the exactly one b-tagged jet and "two or more b-tags" is an event having two or more b-tagged jets.

\begin{tabular}{|c|c|c|c|c|c|}
\hline \hline & & \multicolumn{4}{|c|}{ e+jets } \\
\hline \hline & & $N_{\text {jets }}=1$ & $N_{\text {jets }}=2$ & $N_{\text {jets }}=3$ & $N_{\text {jets }} \geq 4$ \\
\hline \multirow{4}{*}{ before b-tagging } & $N_{l}$ & 22398.0 & 10377.0 & 2592.0 & 618.0 \\
& $N_{t}$ & 15863.0 & 6043.0 & 1300.0 & 320.0 \\
& $N_{t}^{W+t \bar{t}}$ & $14992.4 \pm 221.4$ & $5223.5 \pm 102.9$ & $1031.8 \pm 34.4$ & $259.8 \pm 10.1$ \\
& $N_{t}^{Q C D}$ & $870.6 \pm 221.4$ & $819.5 \pm 102.9$ & $268.2 \pm 34.4$ & $60.2 \pm 10.1$ \\
\hline \multirow{5}{*}{ exactly one b-tag } & $N_{l}^{1 \mathrm{tag}}$ & 606.0 & 650.0 & 284.0 & 130.0 \\
& $N_{t}^{1 \mathrm{tag}}$ & 411.0 & 373.0 & 164.0 & 88.0 \\
& $N_{t}^{1 \mathrm{tag}, \mathrm{W}+\mathrm{t} \overline{\mathrm{t}}}$ & $381.9 \pm 7.9$ & $320.0 \pm 9.3$ & $141.1 \pm 5.2$ & $81.6 \pm 2.6$ \\
& $N_{t}^{1 \mathrm{tag}, \mathrm{QCD}}$ & $29.1 \pm 7.9$ & $53.0 \pm 9.3$ & $22.9 \pm 5.2$ & $6.4 \pm 2.6$ \\
\hline & $N_{l}^{\geq 2 \mathrm{tag}}$ & - & 51.0 & 58.0 & 34.0 \\
& $N_{t}^{\geq 2 \mathrm{tag}}$ & - & 39.0 & 41.0 & 26.0 \\
& $N_{t}^{\geq 2 \operatorname{tag}, \mathrm{W}+\mathrm{t} \overline{\mathrm{t}}}$ & - & $37.8 \pm 1.1$ & $38.6 \pm 1.5$ & $25.2 \pm 0.9$ \\
& $N_{t}^{\geq 2 \mathrm{tag}, \mathrm{QCD}}$ & - & $1.2 \pm 1.1$ & $2.4 \pm 1.5$ & $0.8 \pm 0.9$ \\
\hline \hline
\end{tabular}

Table 5.2: Number of preselected events in the loose and tight samples. The expected contribution from multijet and $W$-like events are obtained by the matrix method for different jet multiplicity bins in the $e+$ jets channel.

The errors on the matrix method results are calculated by the procedure described in Reference [61]. The statistical uncertainties on the number of $N_{t}^{\mathrm{W}+\mathrm{t} \overline{\mathrm{t}}}$ and $N_{t}^{\mathrm{QCD}}$ are defined as follow

$$
\begin{aligned}
\delta N_{t}^{\mathrm{W}+\mathrm{t} \overline{\mathrm{t}}} & =-\delta N_{t}^{\mathrm{QCD}}=\sqrt{\frac{N_{t}^{\mathrm{W}+\mathrm{t} \overline{\mathrm{t}}} \times N_{t}^{\mathrm{QCD}}}{N_{t}}} \\
\delta N_{t}^{\mathrm{W}+\mathrm{t} \overline{\mathrm{t}}}\left(\varepsilon_{s i g}\right) & =-\delta N_{t}^{\mathrm{QCD}}\left(\varepsilon_{s i g}\right)=\frac{d N_{t}^{\mathrm{W}+\mathrm{t} \overline{\mathrm{t}}}}{d \varepsilon_{s i g}} \delta \varepsilon_{s i g}=-\varepsilon_{q c d} \frac{N_{t}-\varepsilon_{q c d} N_{\ell}}{\left(\varepsilon_{s i g}-\varepsilon_{q c d}\right)^{2}} \delta \varepsilon_{s i g} \\
\delta N_{t}^{\mathrm{W}+\mathrm{t} \overline{\mathrm{t}}}\left(\varepsilon_{q c d}\right) & =-\delta N_{t}^{\mathrm{QCD}}\left(\varepsilon_{q c d}\right)=\frac{d N_{t}^{\mathrm{W}+\mathrm{t} \overline{\mathrm{t}}}}{d \varepsilon_{q c d}} \delta \varepsilon_{q c d}=-\varepsilon_{s i g} \frac{\varepsilon_{s i g} N_{\ell}-N_{t}}{\left(\varepsilon_{s i g}-\varepsilon_{q c d}\right)^{2}} \delta \varepsilon_{q c d}
\end{aligned}
$$

where the minus sign indicates that the uncertainties are completely anticorrelated. $\delta N_{t}^{\mathrm{W}+\mathrm{t} \overline{\mathrm{t}}}\left(\varepsilon_{s i g}\right)$ and $\delta N_{t}^{\mathrm{W}+\mathrm{t} \overline{\mathrm{t}}}\left(\varepsilon_{q c d}\right)$ denote the uncertainties coming from the efficiencies and they are derived by error propagation. The total errors for $N_{t}^{\mathrm{W}+\mathrm{t} \overline{\mathrm{t}}}$ and $N_{t}^{\mathrm{QCD}}$ are the quadrature sum of three different uncertainties in Equation 5.7. The calculated errors are shown in Tables 5.2 and 5.3. 


\begin{tabular}{|c|c|c|c|c|c|}
\hline \hline & & \multicolumn{4}{|c|}{$\mu+$ jets } \\
\hline \hline & & $N_{\text {jets }}=1$ & $N_{\text {jets }}=2$ & $N_{\text {jets }}=3$ & $N_{\text {jets }} \geq 4$ \\
\hline \multirow{4}{*}{ before b-tagging } & $N_{l}$ & 15289.0 & 6194.0 & 1389.0 & 388.0 \\
& $N_{t}$ & 13472.0 & 5191.0 & 1120.0 & 306.0 \\
& $N_{t}^{W+t \bar{t}}$ & $13253.1 \pm 90.9$ & $5057.0 \pm 44.0$ & $1078.1 \pm 13.9$ & $295.6 \pm 5.7$ \\
& $N_{t}^{Q C D}$ & $218.9 \pm 90.9$ & $134.0 \pm 44.0$ & $41.9 \pm 13.9$ & $10.4 \pm 5.7$ \\
\hline \multirow{4}{*}{ exactly one b-tag } & $N_{l}^{\text {Itag }}$ & 382.0 & 381.0 & 160.0 & 106.0 \\
& $N_{t}^{1 \mathrm{tag}}$ & 324.0 & 300.0 & 130.0 & 91.0 \\
& $N_{t}^{1 \mathrm{tag}, \mathrm{W}+\mathrm{t} \overline{\mathrm{t}}}$ & $313.2 \pm 4.7$ & $283.2 \pm 6.3$ & $125.6 \pm 2.4$ & $91.7 \pm 1.4$ \\
& $N_{t}^{1 \mathrm{tag}, \mathrm{QCD}}$ & $10.8 \pm 4.7$ & $16.8 \pm 6.3$ & $4.4 \pm 2.4$ & $-0.7 \pm 1.4$ \\
\hline & $N_{l}^{\geq 2 \operatorname{tag}}$ & - & 30.0 & 42.0 & 35.0 \\
two or more b-tags & $N_{t}^{\geq 2 \operatorname{tag}}$ & - & 26.0 & 35.0 & 32.0 \\
& $N_{t}^{\geq 2 \operatorname{tag}, \mathrm{W}+\mathrm{t} \overline{\mathrm{t}}}$ & - & $25.7 \pm 0.5$ & $34.2 \pm 0.9$ & $33.2 \pm 1.2$ \\
& $N_{t}^{\geq 2 \operatorname{tag}, \mathrm{QCD}}$ & - & $0.3 \pm 0.5$ & $0.8 \pm 0.9$ & $-1.2 \pm 1.2$ \\
\hline \hline
\end{tabular}

Table 5.3: Number of preselected events in the loose and tight samples. The expected contribution from multijet and $W$-like events are obtained by the matrix method for different jet multiplicity bins in the $\mu+$ jets channel.

\subsection{Physics Backgrounds}

The contributions of physics backgrounds, except for $\mathrm{W}+$ jets $(W j j, W b b, W c c)$, in the preselected sample are determined as follows

$$
N_{i}^{\text {presel }}=\sigma_{i} \cdot \varepsilon_{i}^{\text {presel, } \text { tot }} \cdot B R_{i} \cdot \mathcal{L}
$$

where $i$ denotes the index of a given background process. In Equation $5.8, \sigma_{i}, \varepsilon_{i}^{\text {presel,tot }}, B R_{i}$ and $\mathcal{L}$ indicate the cross section, preselection efficiency, branching ratio and integrated luminosity for the backgrounds under consideration, respectively. In this analysis, the following backgrounds are estimated using Equation 5.8

- diboson production: $W W \rightarrow l+j e t s, W Z \rightarrow l+j e t s, W Z \rightarrow j j l \bar{l}, Z Z \rightarrow l \bar{l} j j$;

- single top production in $s$-channel and $t$-channel;

- $Z+j e t s: Z j j, Z b b, Z c c$.

The main physics background, $\mathrm{W}+$ jets $(W j j, W b b$, and $W c c)$, is estimated by normalization to the number of events in data sample. The contributions from other backgrounds and the expected 
$t \bar{t}$ signal are obtained using the Equation 5.8 and are subtracted from the number of W-like events determined by the matrix method in each jet multiplicity (denoted as $N_{t}^{\mathrm{W}+\mathrm{t} \overline{\mathrm{t}}}$ in Tables 5.2 and 5.3). The Equation 5.9 shows the formula used to calculate the number of $\mathrm{W}+$ jets events before b-tagging $\left(N_{W+j e t s}^{\text {presel }}\right)$

$$
N_{W+\text { jets }}^{\text {presel }}=N_{t}^{\mathrm{W}+\mathrm{t} \overline{\mathrm{t}}}-\sum_{i} N_{i}^{\text {presel }}-N_{t \bar{t}}^{\text {presel }} .
$$

Tables 5.4 and 5.5 show the contribution of the preselected event sample. The number of expected events for the $t \bar{t}$ signal follows from the Equation 5.8 in the tables. The theoretical $t \bar{t}$ cross section $(6.8 \mathrm{pb})[7]$ is used in the tables. However, the $t \bar{t}$ contributions are allowed to float in the cross section calculation and the results here are used as an example for control plots that are described in Section 5.4. The cross section calculation will be discussed in Chapter 6 .

The number of $\mathrm{W}+$ jets events in the tables are further broken down into $\mathrm{Wjj}$, Wbb and Wcc contributions using the ALPGEN cross sections. To give the relative normalization of these processes, the Wbb and Wcc NLO cross sections are multiplied by a heavy flavor scale factor of $1.17 \pm 0.18$ to account for NLO corrections that are not provided by the ALPGEN. The heavy flavor scale factor is obtained by comparing MC and data samples for lepton +2 jets events [51]. The relative normalization factor is calculated using the Equation 5.10

$$
f_{\text {norm }}=\frac{N_{W+\text { jets }}^{\text {presel }}}{\bar{N}_{W j j}^{\text {presel }}+1.17 \cdot\left(\bar{N}_{W b b}^{\text {presel }}+\bar{N}_{W c c}^{\text {presel }}\right)}
$$

where $\bar{N}_{W j j}^{\text {presel }}, \bar{N}_{W b b}^{\text {presel }}$, and $\bar{N}_{W c c}^{\text {presel }}$ are the number of expected events that is calculated using the Equation 5.8 and the ALPGEN cross sections, respectively. Using this normalization factor, the contributions of $\mathrm{Wjj}$, Wbb and Wcc can be determined as follows

$$
\begin{aligned}
& N_{W j j}^{\text {presel }}=f_{\text {norm }} \cdot \bar{N}_{W j j}^{\text {presel }} \\
& N_{W b b}^{\text {presel }}=f_{\text {norm }} \cdot 1.17 \cdot \bar{N}_{W b b}^{\text {presel }} \\
& N_{W c c}^{\text {presel }}=f_{\text {norm }} \cdot 1.17 \cdot \bar{N}_{W c c}^{\text {presel }} .
\end{aligned}
$$


The errors for $\mathrm{W}+$ jets contribution are determined by two sources: the matrix method and K-factor uncertainty and are calculated using Equation 5.12.

$$
\begin{aligned}
& \delta N_{W+\text { jets }}^{\text {presel }}=\sqrt{\delta_{1}^{2}+\delta_{2}^{2}} \\
& \delta_{1}=\frac{N_{W+\text { jets }}^{\text {presel }}}{N_{t}^{\mathrm{W}+\mathrm{t} \overline{\mathrm{t}}}} \cdot \delta N_{t}^{\mathrm{W}+\mathrm{t} \overline{\mathrm{t}}} \\
& \delta_{2}=\frac{\Delta\left(N_{W+\text { jets }}^{1.17+0.18}-N_{W+\text { jets }}^{1.17-0.18}\right)}{2}
\end{aligned}
$$

where $N_{W+j e t s}^{1.17+0.18}$ and $N_{W+j e t s}^{1.17-0.18}$ indicate the number of events recalculated by varying the K-factor by $\pm 1 \sigma$ errors $( \pm 0.18)$. In Tables 5.4 and 5.5 , the errors for other physics processes are not calculated because the contributions are measured using the huge statistics MC samples as well as are very smaller than the contributions of $\mathrm{W}+$ jets.

\begin{tabular}{|c|c|c|c|c|}
\hline \hline & \multicolumn{4}{|c|}{$\mathrm{e}+\mathrm{jets}$} \\
\hline & $1 j e t$ & $2 j e t s$ & $3 j e t s$ & $\geq 4 j e t s$ \\
\hline \hline$W j j$ & 13131.3 & 3802.9 & 560.9 & 69.0 \\
$W b b$ & 344.7 & 217.6 & 53.1 & 7.9 \\
$W c c$ & 1031.0 & 618.9 & 131.8 & 16.3 \\
\hline$W+$ jets & $14507.0 \pm 214.2$ & $4639.4 \pm 91.4$ & $745.8 \pm 24.9$ & $93.2 \pm 3.6$ \\
\hline$t b$ & 5.3 & 14.5 & 4.2 & 0.8 \\
$t q b$ & 12.7 & 26.6 & 8.6 & 2.2 \\
\hline$Z j j$ & 285.5 & 225.5 & 62.2 & 19.0 \\
$Z b b$ & 11.8 & 14.1 & 6.4 & 2.1 \\
$Z c c$ & 20.3 & 24.4 & 10.4 & 3.5 \\
\hline$W W$ & 113.2 & 155.0 & 33.3 & 5.9 \\
$W Z$ & 16.0 & 26.2 & 6.4 & 1.2 \\
$Z Z$ & 1.4 & 1.6 & 0.9 & 0.2 \\
\hline$t \bar{t} \rightarrow l l$ & 14.1 & 46.6 & 22.9 & 5.0 \\
$t \bar{t} \rightarrow l+$ jets & 5.0 & 49.6 & 130.7 & 126.6 \\
\hline$M u l t i j e t$ & $870.6 \pm 221.4$ & $819.5 \pm 102.9$ & $268.2 \pm 34.4$ & $60.2 \pm 10.1$ \\
\hline total & 15863.0 & 6043.0 & 1300.0 & 320.0 \\
\hline \hline data & 15863.0 & 6043.0 & 1300.0 & 320.0 \\
\hline \hline
\end{tabular}

Table 5.4: Summary of event yields before $b$-tagging in the $e+$ jets channel. 


\begin{tabular}{|c|c|c|c|c|}
\hline \hline & \multicolumn{4}{|c|}{$\mu+$ jets } \\
\hline & $1 j e t$ & $2 j e t s$ & $3 j e t s$ & $\geq 4$ jets \\
\hline \hline$W j j$ & 11162.9 & 3659.1 & 612.6 & 113.9 \\
$W b b$ & 281.4 & 206.5 & 57.5 & 12.5 \\
$W c c$ & 849.5 & 580.2 & 153.4 & 24.6 \\
\hline$W+$ jets & $12293.9 \pm 84.3$ & $4445.8 \pm 38.7$ & $823.5 \pm 10.6$ & $151.1 \pm 2.9$ \\
\hline$t b$ & 2.9 & 9.5 & 2.9 & 0.6 \\
$t q b$ & 10.1 & 22.1 & 7.1 & 1.8 \\
\hline$Z j j$ & 765.5 & 308.2 & 76.8 & 21.6 \\
$Z b b$ & 12.6 & 14.2 & 5.9 & 1.7 \\
$Z c c$ & 55.0 & 38.0 & 12.5 & 3.7 \\
\hline$W W$ & 85.6 & 124.6 & 27.1 & 5.2 \\
$W Z$ & 14.0 & 25.1 & 6.0 & 1.1 \\
$Z Z$ & 2.0 & 3.6 & 1.0 & 0.2 \\
\hline$t \bar{t} \rightarrow l l$ & 9.0 & 35.4 & 18.2 & 3.9 \\
$t \bar{t} \rightarrow l+$ jets & 2.6 & 30.5 & 97.1 & 104.9 \\
\hline$M u l t i j e t$ & $218.9 \pm 90.9$ & $134.0 \pm 44.0$ & $41.9 \pm 13.9$ & $10.4 \pm 5.7$ \\
\hline total & 13472.0 & 5191.0 & 1120.0 & 306.0 \\
\hline \hline data & 13472.0 & 5191.0 & 1120.0 & 306.0 \\
\hline \hline
\end{tabular}

Table 5.5: Summary of event yields before $b$-tagging in the $\mu+$ jets channel.

\subsection{Background Estimation in b-tagged Events}

In b-tagged events, the event tagging probability for each jet multiplicity is evaluated. It is multiplied by the estimated number of preselected events which are discussed in the previous section in order to obtain the expected number of tagged events

$$
N_{i}^{\text {tag }}=N_{i}^{\text {presel }} P_{i}^{\text {tag }}
$$

where $i$ denotes the index of a given background process and $P_{i}^{t a g}$ is the event tagging probability for each jet multiplicity.

The b-id group provides the b-tagging efficiency of a jet depending on jet $p_{\mathrm{T}}$ and $\eta$ as well as jet flavors $\left(b, c\right.$ and light-quark) [34]. The probability of tagging a given jet $P_{j e t(\alpha)}$ is the product of the taggability $P^{\text {taggability }}[62]$ and tagging efficiency $\varepsilon_{\alpha}$ for the jet

$$
P_{j e t(\alpha)}=P^{\text {taggability }} \varepsilon_{\alpha}
$$


where $\alpha$ denotes the flavor of the jet. The event probability to have at least one b-tagged jet is then

$$
P_{\text {event }}^{\text {tag }}(n \geq 1)=1-\prod_{i=1}^{N_{\text {jets }}}\left(1-P_{\text {jet }\left(\alpha_{i}\right)}\right)
$$

where $N_{\text {jets }}$ represents the number of jets with $p_{T}>20 \mathrm{GeV}$ and $|\eta|<2.5$ in the event. The probability to have exactly one b-tagged jet is

$$
P_{\text {event }}^{\text {tag }}(n=1)=\sum_{j=1}^{N_{\text {jets }}} P_{j e t}\left(\alpha_{j}\right) \prod_{i=1 ; i \neq j}^{N_{j e t s}}\left(1-P_{j e t}\left(\alpha_{i}\right)\right)
$$

and hence the probability to have two or more b-tagged jets is

$$
P_{\text {event }}^{\text {tag }}(n \geq 2)=P_{\text {event }}^{\text {tag }}(n \geq 1)-P_{\text {event }}^{\text {tag }}(n=1)
$$

These event probabilities can be applied to Equation 5.13.

The number of expected tagged events for signal and background contributions are shown in Tables $5.6 \sim 5.9$. The results are split into "exactly one b-tag" (Tables 5.6 and 5.7) and "two or more b-tags" (Tables 5.8 and 5.9). The difference in the tables describes the relative error between the number of data events and the total number of expected events.

\subsection{Control Plots}

Using the estimated number of events for $t \bar{t}$ signal (using the theoretical cross section $6.8 \mathrm{pb}$ ) and background contribution, so-called "control plots" showing the distributions for kinematic variables are generated in order to check for any discrepancies between the MC model of the data and the actual data. These distributions are very important because they show that the method used to estimate backgrounds is accurately reproducing the features of the real data. Distributions of the four main kinematic variables (lepton $p_{\mathrm{T}}, \mathbb{E}_{T}$, leading jet $p_{\mathrm{T}}$, and $\mathrm{W}$ transverse momentum) are shown in Figures $5.1 \sim 5.8$, respectively. Control plots for other kinematic variables are shown in Appendix C. There are no significant disagreements between the MC and the data sample for these 


\begin{tabular}{|c|c|c|c|c|}
\hline \hline & \multicolumn{4}{|c|}{ e+jets } \\
\hline & $1 j e t$ & $2 j e t s$ & $3 j e t s$ & $\geq 4 j e t s$ \\
\hline \hline$W j j$ & 176.1 & 74.6 & 14.1 & 2.2 \\
$W b b$ & 109.6 & 79.5 & 20.1 & 3.0 \\
$W c c$ & 61.7 & 59.8 & 16.1 & 2.4 \\
\hline$W+$ jets & $347.5 \pm 22.0$ & $213.8 \pm 16.1$ & $50.4 \pm 4.0$ & $7.5 \pm 0.6$ \\
\hline$t b$ & 2.5 & 6.8 & 2.0 & 0.4 \\
$t q b$ & 4.9 & 11.8 & 3.9 & 1.0 \\
\hline$Z j j$ & 1.4 & 2.4 & 1.1 & 0.5 \\
$Z b b$ & 2.6 & 4.8 & 2.4 & 0.9 \\
$Z c c$ & 1.1 & 2.3 & 1.2 & 0.5 \\
\hline$W W$ & 3.9 & 9.0 & 2.4 & 0.6 \\
$W Z$ & 1.6 & 3.1 & 0.8 & 0.2 \\
$Z Z$ & 0.1 & 0.2 & 0.1 & 0.0 \\
\hline$t \bar{t} \rightarrow l l$ & 6.6 & 21.8 & 10.7 & 2.3 \\
$t \bar{t} \rightarrow l+$ jets & 1.6 & 22.1 & 60.2 & 57.8 \\
\hline$M u l t i j e t$ & $29.1 \pm 7.9$ & $53.0 \pm 9.3$ & $22.9 \pm 5.2$ & $6.4 \pm 2.6$ \\
\hline total & $402.8 \pm 23.4$ & $351.2 \pm 18.6$ & $158.1 \pm 6.6$ & $78.0 \pm 2.7$ \\
\hline \hline data & 411.0 & 373.0 & 164.0 & 88.0 \\
\hline \hline difference & $+2.0 \%$ & $+5.8 \%$ & $+3.6 \%$ & $+11.4 \%$ \\
\hline \hline
\end{tabular}

Table 5.6: Summary of event yields with exactly one $b$-tag in the $e+$ jets channel.

\begin{tabular}{|c|c|c|c|c|}
\hline \hline & \multicolumn{4}{|c|}{$\mu+$ jets } \\
\hline & $1 j e t$ & $2 j e t s$ & $3 j e t s$ & $\geq 4 j e t s$ \\
\hline \hline$W j j$ & 146.3 & 74.0 & 15.9 & 4.0 \\
$W b b$ & 91.5 & 77.5 & 22.3 & 4.8 \\
$W c c$ & 49.8 & 55.2 & 19.5 & 3.7 \\
\hline$W+$ jets & $287.7 \pm 17.8$ & $206.7 \pm 14.8$ & $57.8 \pm 4.2$ & $12.5 \pm 0.9$ \\
\hline$t b$ & 1.4 & 4.4 & 1.4 & 0.3 \\
$t q b$ & 4.0 & 10.0 & 3.2 & 0.8 \\
\hline$Z j j$ & 5.8 & 4.0 & 1.5 & 0.5 \\
$Z b b$ & 3.2 & 5.1 & 2.3 & 0.7 \\
$Z c c$ & 3.8 & 4.2 & 1.5 & 0.5 \\
\hline$W W$ & 3.1 & 7.5 & 2.0 & 0.5 \\
$W Z$ & 1.4 & 2.8 & 0.7 & 0.2 \\
$Z Z$ & 0.2 & 0.4 & 0.1 & 0.0 \\
\hline$t \bar{t} \rightarrow l l$ & 4.4 & 16.5 & 8.5 & 1.8 \\
$t \bar{t} \rightarrow l+$ jets & 0.8 & 13.6 & 44.8 & 47.7 \\
\hline$M u l t i j e t$ & $10.8 \pm 4.7$ & $16.8 \pm 6.3$ & $4.4 \pm 2.4$ & $-0.7 \pm 1.4$ \\
\hline total & $326.5 \pm 18.4$ & $292.0 \pm 16.1$ & $128.2 \pm 4.9$ & $64.8 \pm 1.6$ \\
\hline \hline data & 324.0 & 300.0 & 130.0 & 91.0 \\
\hline \hline difference & $-0.8 \%$ & $+2.7 \%$ & $+1.4 \%$ & $+28.8 \%$ \\
\hline \hline
\end{tabular}

Table 5.7: Summary of event yields with exactly one $b$-tag in the $\mu+$ jets channel. 


\begin{tabular}{|c|c|c|c|c|}
\hline \hline & \multicolumn{4}{|c|}{ e+jets } \\
\hline & $1 j e t$ & $2 j e t s$ & $3 j e t s$ & $\geq 4 j e t s$ \\
\hline \hline$W j j$ & - & 0.3 & 0.1 & 0.0 \\
$W b b$ & - & 12.4 & 3.8 & 0.6 \\
$W c c$ & - & 1.8 & 0.7 & 0.2 \\
\hline$W+$ jets & - & $14.5 \pm 1.8$ & $4.7 \pm 0.5$ & $0.8 \pm 0.1$ \\
\hline$t b$ & - & 2.8 & 0.9 & 0.2 \\
$t q b$ & - & 0.5 & 0.8 & 0.3 \\
\hline$Z j j$ & - & 0.0 & 0.0 & 0.0 \\
$Z b b$ & - & 0.4 & 0.4 & 0.2 \\
$Z c c$ & - & 0.1 & 0.1 & 0.0 \\
\hline$W W$ & - & 0.1 & 0.1 & 0.1 \\
$W Z$ & - & 0.7 & 0.2 & 0.0 \\
$Z Z$ & - & 0.0 & 0.0 & 0.0 \\
\hline$t \bar{t} \rightarrow l l$ & - & 8.3 & 4.7 & 1.1 \\
$t \bar{t} \rightarrow l+j e t s$ & - & 3.8 & 22.0 & 28.6 \\
\hline$M u l t i j e t$ & - & $1.2 \pm 1.1$ & $2.4 \pm 1.5$ & $0.8 \pm 0.9$ \\
\hline total & - & $32.4 \pm 2.1$ & $36.2 \pm 1.6$ & $32.1 \pm 0.9$ \\
\hline \hline data & - & 39.0 & 41.0 & 26.0 \\
\hline \hline difference & - & $+16.8 \%$ & $+11.6 \%$ & $-23.5 \%$ \\
\hline \hline
\end{tabular}

Table 5.8: Summary of event yields with two or more $b$-tags in the $e+$ jets channel.

\begin{tabular}{|c|c|c|c|c|}
\hline \hline & \multicolumn{4}{|c|}{$\mu+$ jets } \\
\hline & $1 j e t$ & $2 j e t s$ & $3 j e t s$ & $\geq 4 j e t s$ \\
\hline \hline$W j j$ & - & 0.3 & 0.1 & 0.1 \\
$W b b$ & - & 12.5 & 4.4 & 1.1 \\
$W c c$ & - & 1.5 & 1.0 & 0.3 \\
\hline$W+$ jets & - & $14.4 \pm 1.8$ & $5.5 \pm 0.6$ & $1.4 \pm 0.2$ \\
\hline$t b$ & - & 1.9 & 0.6 & 0.1 \\
$t q b$ & - & 0.4 & 0.7 & 0.3 \\
\hline$Z j j$ & - & 0.0 & 0.0 & 0.0 \\
$Z b b$ & - & 0.5 & 0.4 & 0.2 \\
$Z c c$ & - & 0.1 & 0.1 & 0.0 \\
\hline$W W$ & - & 0.1 & 0.1 & 0.0 \\
$W Z$ & - & 0.6 & 0.2 & 0.1 \\
$Z Z$ & - & 0.1 & 0.0 & 0.0 \\
\hline$t \bar{t} \rightarrow l l$ & - & 6.7 & 3.9 & 0.9 \\
$t \bar{t} \rightarrow l+j \mathrm{jets}$ & - & 2.4 & 17.3 & 25.1 \\
\hline$M u l t i j e t$ & - & $0.3 \pm 0.5$ & $0.8 \pm 0.9$ & $-1.2 \pm 1.2$ \\
\hline total & - & $27.5 \pm 1.8$ & $29.5 \pm 1.1$ & $27.0 \pm 1.2$ \\
\hline \hline data & - & 26.0 & 35.0 & 32.0 \\
\hline \hline difference & - & $-5.6 \%$ & $+15.7 \%$ & $+15.8 \%$ \\
\hline \hline
\end{tabular}

Table 5.9: Summary of event yields with two or more $b$-tags in the $\mu+$ jets channel. 
distributions of kinematic variables.
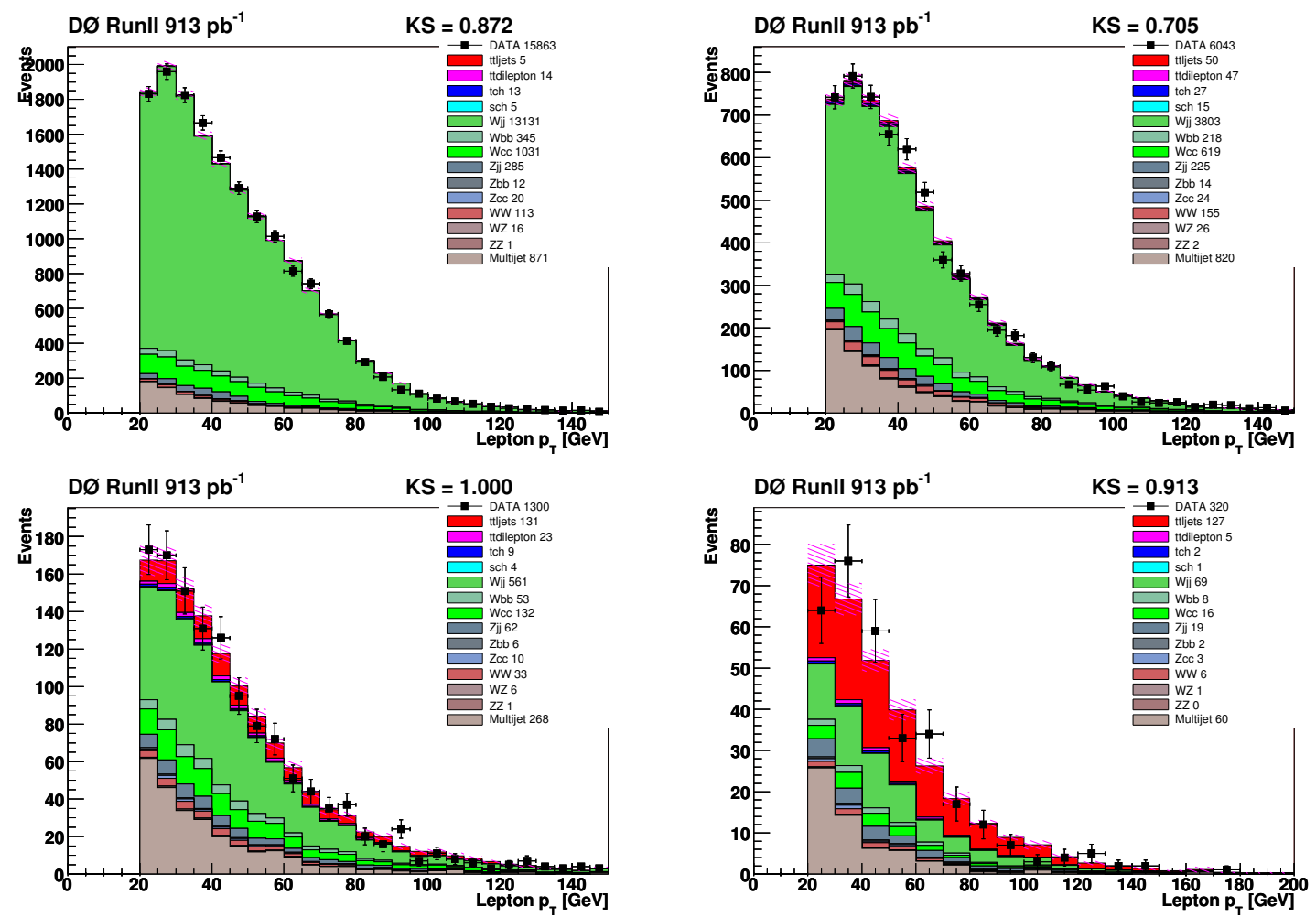

Figure 5.1: Electron $p_{\mathrm{T}}$ distributions for the preselected sample in the $e+$ jets channel. The plots show the results for different jet multiplicities: $=1$ jet (top left), $=2$ jets (top right), $=3$ jets (bottom left), and $\geq 4$ jets (bottom right). 

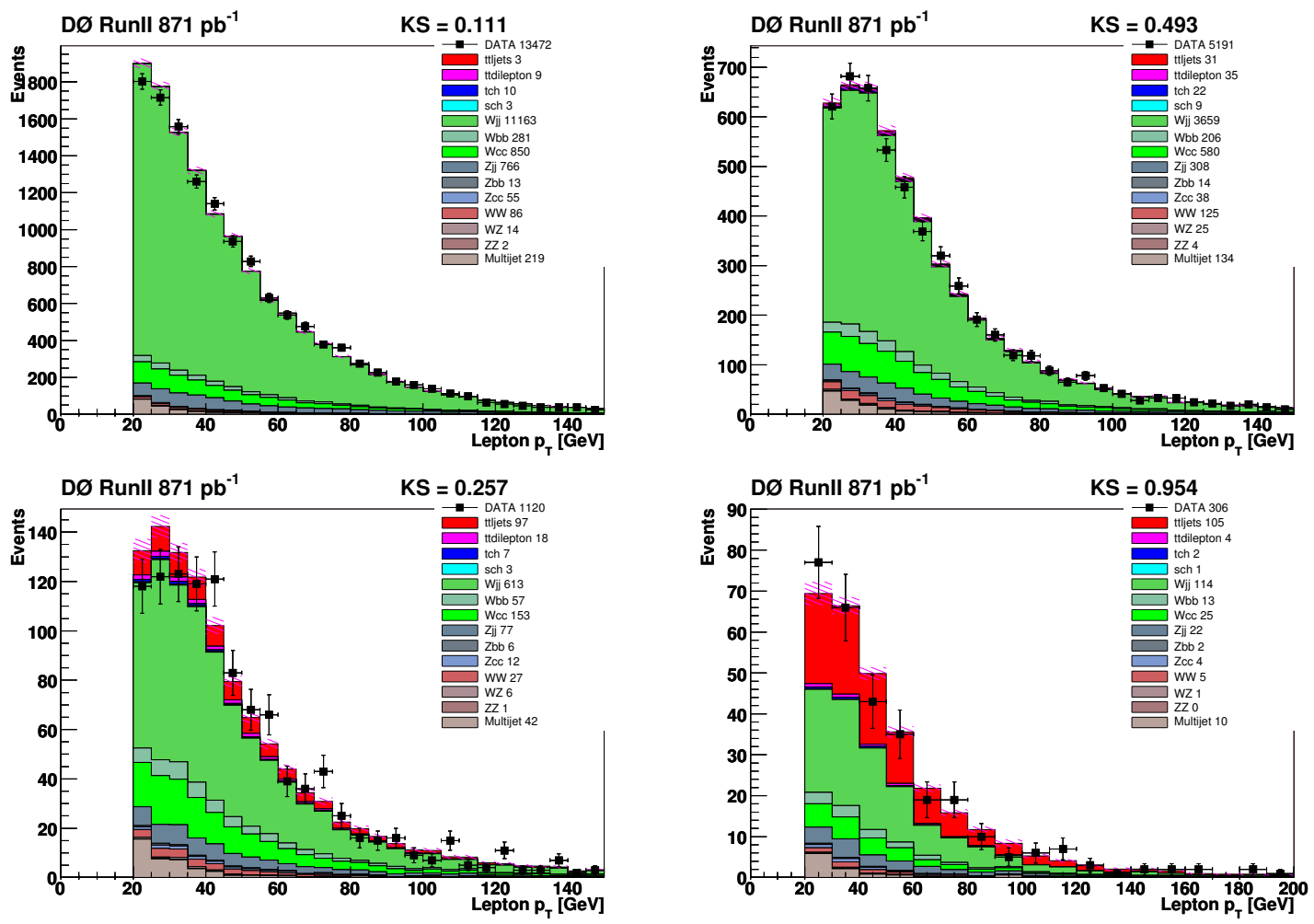

Figure 5.2: Muon $p_{\mathrm{T}}$ distributions for the preselected sample in the $\mu+$ jets channel. The plots show the results for different jet multiplicities: $=1$ jet (top left), $=2$ jets (top right), $=3$ jets (bottom left), and $\geq 4$ jets (bottom right). 

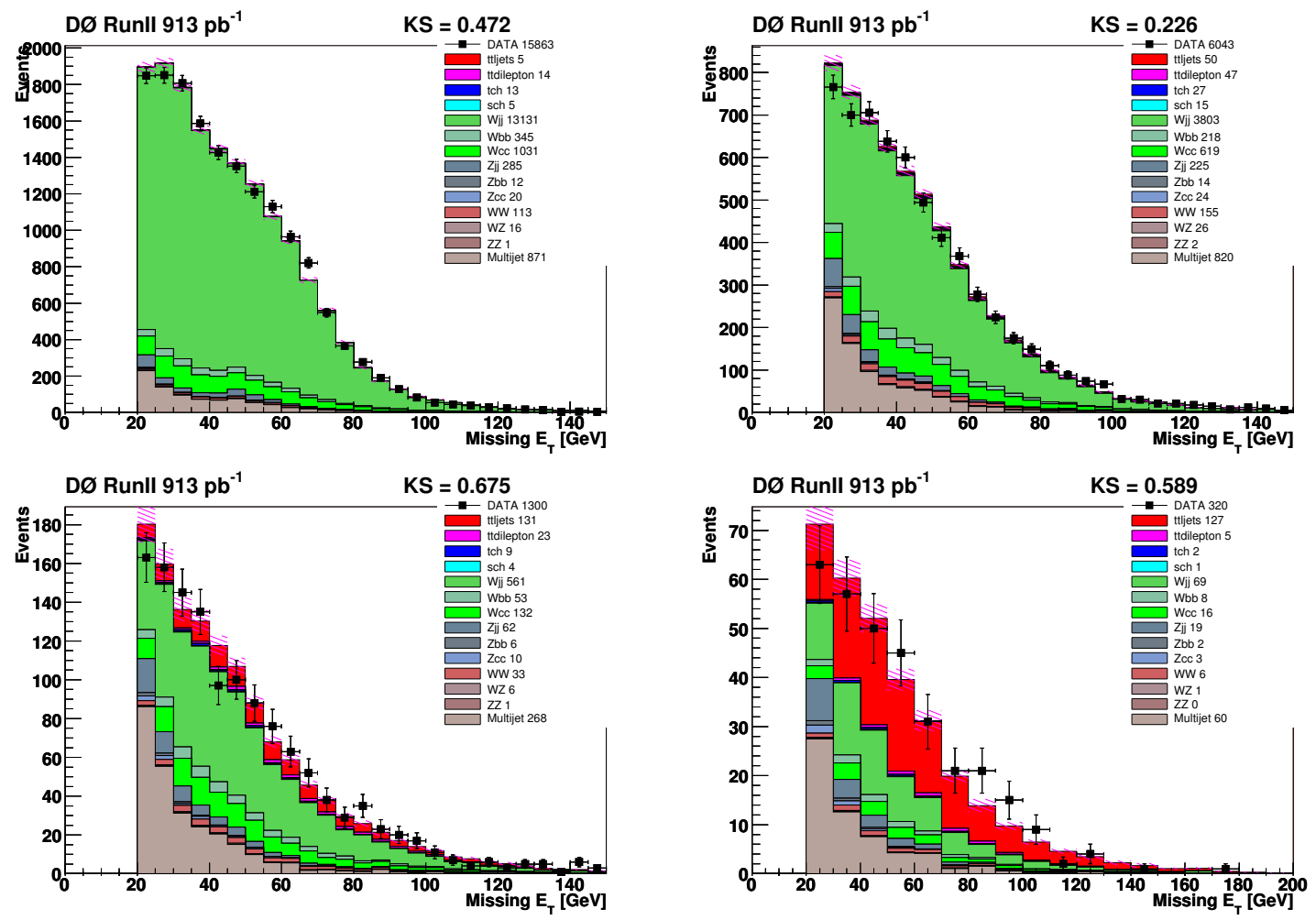

Figure 5.3: $\mathbb{E}_{T}$ distributions for the preselected sample in the $e+$ jets channel. The plots show the results for different jet multiplicities: $=1$ jet (top left), $=2$ jets (top right), $=3$ jets (bottom left), and $\geq 4$ jets (bottom right). 

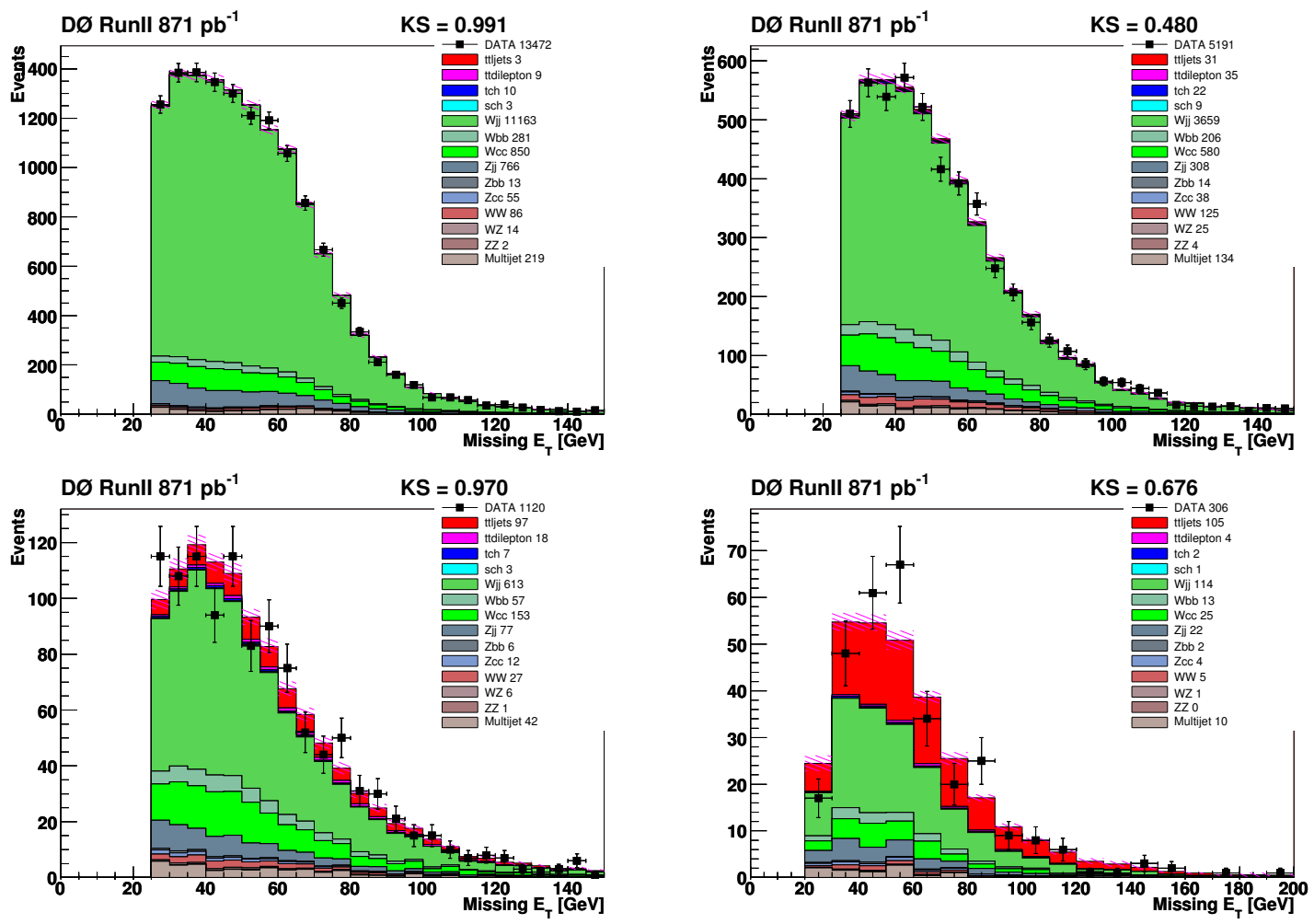

Figure 5.4: $E_{T}$ distributions for the preselected sample in the $\mu+$ jets channel. The plots show the results for different jet multiplicities: $=1$ jet (top left), $=2$ jets (top right), $=3$ jets (bottom left), and $\geq 4$ jets (bottom right). 

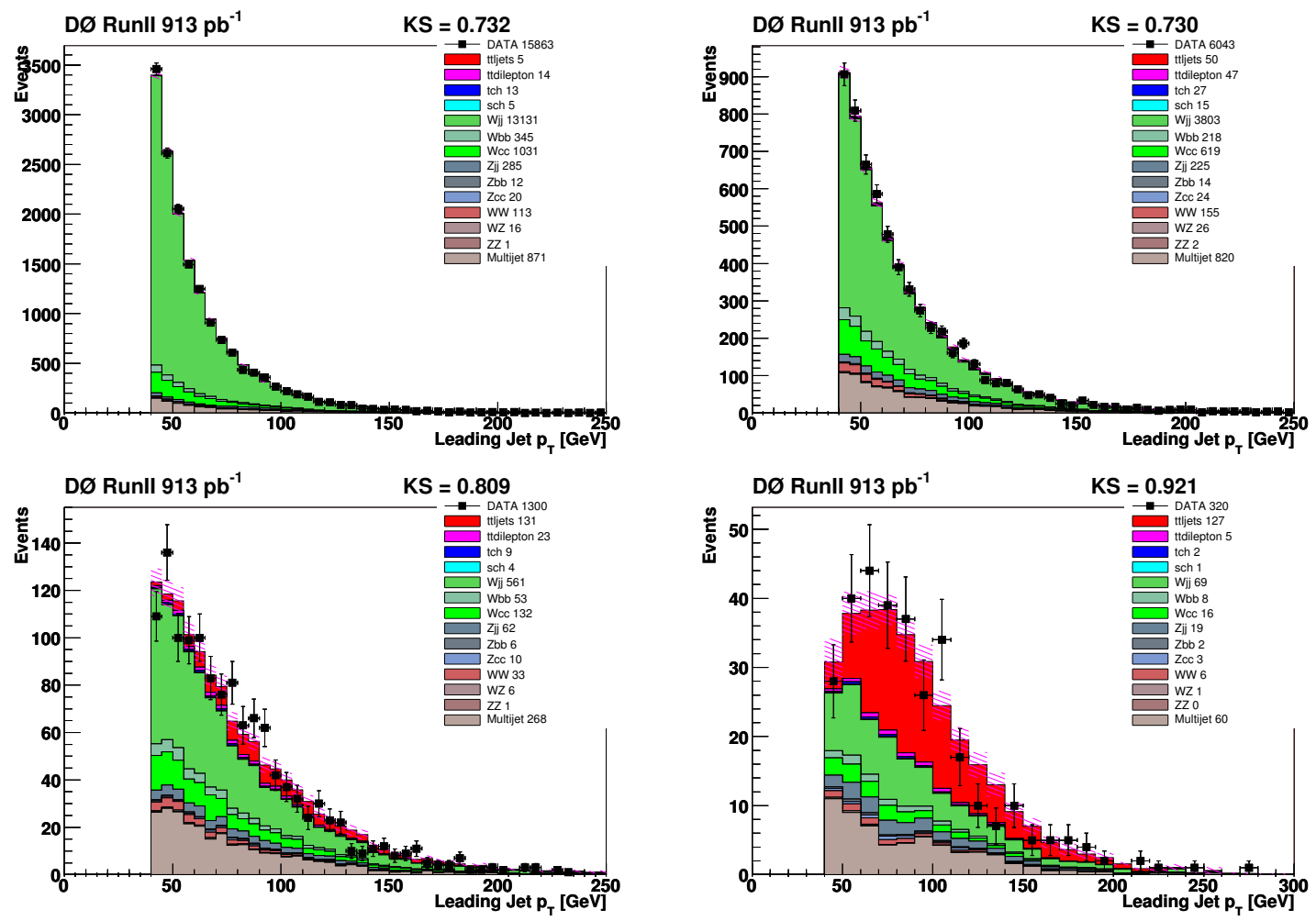

Figure 5.5: Leading jet $p_{\mathrm{T}}$ distributions for the preselected sample in the $e+$ jets channel. The plots show the results for different jet multiplicities: $=1$ jet (top left), $=2$ jets (top right), $=3$ jets (bottom left), and $\geq 4$ jets (bottom right). 

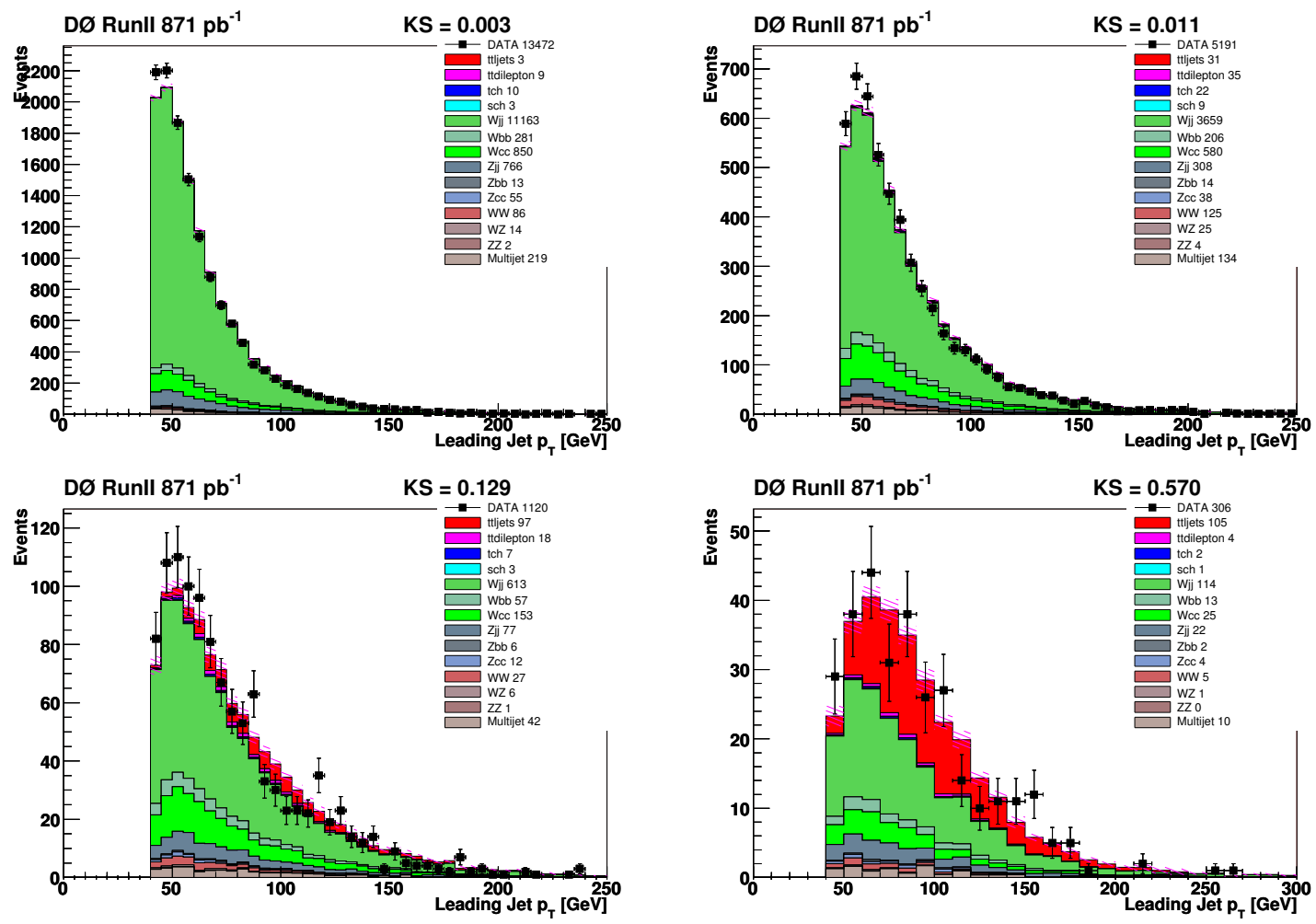

Figure 5.6: Leading jet $p_{\mathrm{T}}$ distributions for the preselected sample in the $\mu+$ jets channel. The plots show the results for different jet multiplicities: $=1$ jet (top left), $=2$ jets (top right), $=3$ jets (bottom left), and $\geq 4$ jets (bottom right). 

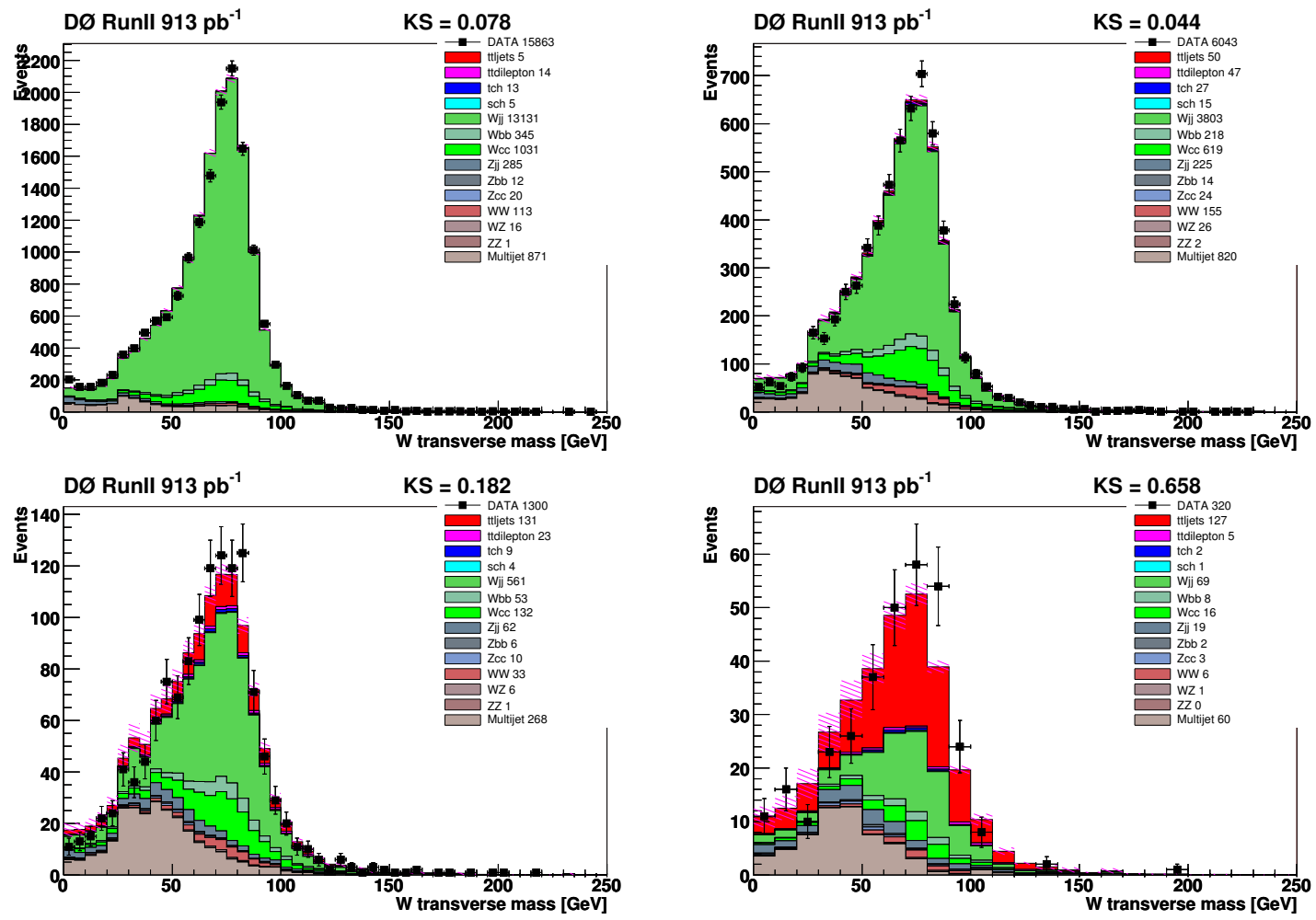

Figure 5.7: The $e \nu$ transverse mass distributions for the preselected sample in the $e+$ jets channel. The plots show the results for different jet multiplicities: $=1$ jet (top left), $=2$ jets (top right), $=3$ jets (bottom left), and $\geq 4$ jets (bottom right). 

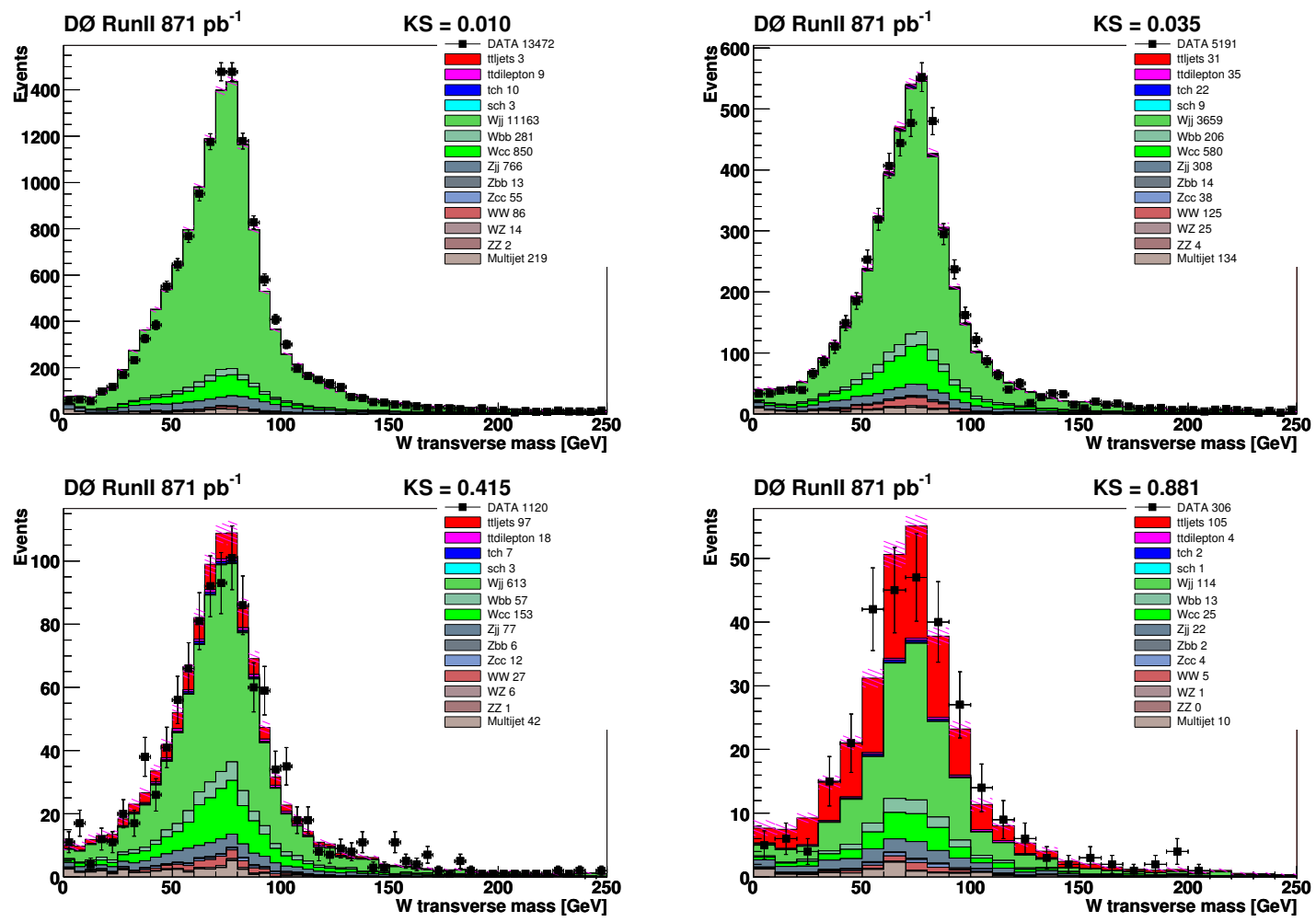

Figure 5.8: The $\mu \nu$ transverse mass distributions for the preselected sample in the $\mu+$ jets channel. The plots show the results for different jet multiplicities: $=1$ jet (top left), $=2$ jets (top right), $=3$ jets (bottom left), and $\geq 4$ jets (bottom right). 


\section{Chapter 6}

\section{Cross Section Calculation}

This analysis is a counting experiment. Therefore, the cross section of the top quark pair production is extracted after subtracting backgrounds estimated from data. To calculate the cross section, a maximum likelihood fit is performed to the observed number of events. In this chapter, it is described how to extract the cross section and the result is presented.

\subsection{Procedure}

The likelihood function $\mathcal{L}$ used to calculate the top quark pair production cross section $\sigma_{t \bar{t}}$ is given by

$$
\mathcal{L}=\prod_{\gamma} \mathcal{P}\left(N_{\gamma}^{\text {obs }}, N_{\gamma}^{\text {predicted }}\left(\sigma_{t \bar{t}}\right)\right)
$$

where $\gamma$ indicates the channel and $N_{\gamma}^{\text {obs }}$ denotes the number of observed events in the channel $\gamma$. This analysis has eight different channels: $e+$ jets $(3$ jets $), e+$ jets $(4$ or more jets), $\mu+$ jets $(3$ jets $)$, and $\mu+$ jets $(4$ or more jets $)$ with exactly one b-tag and two or more b-tags. $\mathcal{P}\left(N^{\text {obs }}, N^{\text {predicted }}\right)$ denotes the Poisson probability to observe $N^{\text {obs }}$ events when the number of predicted events is $N^{\text {predicted }}$. The number of predicted events is the sum of the number of estimated background events $(\mathrm{W}+\mathrm{jets}$, $\mathrm{Z}+$ jets, single top, diboson, and multijet) and the number of expected signal events $(t \bar{t} \rightarrow l+$ jets 
and $t \bar{t} \rightarrow l l)$ which are proportional to the $t \bar{t}$ cross section. It is assumed that the cross section is the same in the $t \bar{t} \rightarrow l+$ jets and $t \bar{t} \rightarrow l l$ channels. The $t \bar{t}$ cross section is determined by maximizing the likelihood function in Equation 6.1. The number of predicted background events also depend on $\sigma_{t \bar{t}}$ because the number of $\mathrm{W}+$ jets events are normalized to the data after subtracting the number of expected events for signal and other background, as shown in Equation 5.9.

The matrix method, which is discussed in Section 5.1, is applied three times in this analysis for both untagged and tagged samples.

1. In the untagged sample (rejected by b-tagging), the solutions of the matrix method are

$$
\begin{aligned}
N_{W+t \bar{t}}^{0 \mathrm{tag}} & =\varepsilon_{\text {sig }} \frac{\tilde{N}_{\text {tight }}^{0 \mathrm{tag}}-\varepsilon_{Q C D} \tilde{N}_{\text {loose }}^{0 \mathrm{tag}}}{\varepsilon_{\text {sig }}-\varepsilon_{Q C D}} \\
N_{Q C D}^{0 \mathrm{tag}} & =\varepsilon_{Q C D} \frac{\varepsilon_{\text {sig }} \tilde{N}_{\text {loose }}^{0 \mathrm{tag}}-\tilde{N}_{\text {tight }}^{0 \mathrm{tag}}}{\varepsilon_{\text {sig }}-\varepsilon_{Q C D}}
\end{aligned}
$$

where $N_{W+t \bar{t}}^{0 \text { tag }}$ and $N_{Q C D}^{0 \mathrm{tag}}$ are the number of events with true and fake isolated leptons in the untagged tight sample. Otag indicates the number of events in untagged sample shown in Table 5.2 and 5.3. The tilde denotes it is an observed number in this analysis. Therefore, $\tilde{N}_{\text {loose }}^{0 t a g}$ and $\tilde{N}_{\text {tight }}^{0 t a g}$ are the number of events observed in the loose and the tight untagged samples, respectively. In addition, $\tilde{N}_{\text {loose }}^{0 \text { tag }}$ and $\tilde{N}_{\text {tight }}^{0 t a g}$ are not independent variables because $\tilde{N}_{\text {tight }}^{0 \text { tag }}$ is a subsample of $\tilde{N}_{\text {loose }}^{0 \text { tag }}$. To solve this problem, $\tilde{N}_{\text {loose }}^{\text {otag }}$ can be split into $\tilde{N}_{\text {tight }}^{0 \text { tag }}$ and $\tilde{N}_{\text {loose-tight }}^{0 \text { tag }}$. The Equation 6.2 can be re-expressed by these independent variables as follows

$$
\begin{aligned}
N_{W+t \bar{t}}^{0 \mathrm{tag}} & =\varepsilon_{\text {sig }} \frac{\tilde{N}_{\text {tight }}^{0 \mathrm{tag}}-\varepsilon_{Q C D}\left(\tilde{N}_{\text {tight }}^{\mathrm{tag}}+\tilde{N}_{\text {loose-tight }}^{\mathrm{tag}}\right)}{\varepsilon_{\text {sig }}-\varepsilon_{Q C D}} \\
N_{Q C D}^{0 \mathrm{tag}} & =\varepsilon_{Q C D} \frac{\varepsilon_{\text {sig }}\left(\tilde{N}_{\text {tight }}^{0 \mathrm{tag}}+\tilde{N}_{\text {loose-tight }}^{\mathrm{ttag}}\right)-\tilde{N}_{\text {tight }}^{0 \mathrm{tag}}}{\varepsilon_{\text {sig }}-\varepsilon_{Q C D}} .
\end{aligned}
$$

The number of observed events, $\tilde{N}_{\text {tight }}^{0 \text { tag }}$ and $\tilde{N}_{\text {loose-tight }}^{0 \text { tag }}$, are put into the likelihood function, $\mathcal{L}_{0}$

$$
\mathcal{L}_{0}=\mathcal{P}\left(\tilde{\mathrm{N}}_{\text {tight }}^{0 \text { tag }}, \mathrm{N}_{\text {tight }}^{\text {0tag }}\right) \times \mathcal{P}\left(\tilde{\mathrm{N}}_{\text {loose-tight }}^{0 \text { tag }}, \mathrm{N}_{\text {loose-tight }}^{0 \text { tag }}\right)
$$


where $N_{\text {tight }}^{0 \text { tag }}$ and $N_{\text {loose-tight }}^{0 \text { tag }}$ without the tilde denote the true values. The Poisson probability $\mathcal{P}$ is the probability to observe $\tilde{N}_{\text {tight }}^{0 t a g}\left(\tilde{N}_{\text {loose-tight }}^{\text {otag }}\right)$ events given the true value $N_{\text {tight }}^{0 \text { tag }}$ $\left(N_{\text {loose-tight }}^{0 \text { tag }}\right) \cdot N_{\text {tight }}^{0 \text { tag }}$ and $N_{\text {loose-tight }}^{\text {tag }}$ are allowed to float at each step of the maximization procedure to find the best estimate of their true values.

2. The solution of the matrix method in the exactly one b-tag sample can be derived by same formula as Equation 6.2

$$
\begin{aligned}
N_{W+t \bar{t}}^{1 \mathrm{tag}} & =\varepsilon_{\text {sig }} \frac{\tilde{N}_{\text {tight }}^{1 \mathrm{tag}}-\varepsilon_{Q C D}\left(\tilde{N}_{\text {tight }}^{1 \mathrm{tag}}+\tilde{N}_{\text {loose-tight }}^{1 \mathrm{tag}}\right)}{\varepsilon_{\text {sig }}-\varepsilon_{Q C D}} \\
N_{Q C D}^{1 \mathrm{tag}} & =\varepsilon_{Q C D} \frac{\varepsilon_{\text {sig }}\left(\tilde{N}_{\text {tight }}^{1 \mathrm{tag}}+\tilde{N}_{\text {loose-tight }}^{1 \mathrm{tag}}\right)-\tilde{N}_{\text {tight }}^{1 \mathrm{tag}}}{\varepsilon_{\text {sig }}-\varepsilon_{Q C D}} .
\end{aligned}
$$

In Equation 6.5, the numbers of observed loose-tight and tight events in the exactly one b-tag samples, $\tilde{N}_{\text {loose-tight }}^{1 \text { tag }}$ and $\tilde{N}_{\text {tight }}^{1 \text { tag }}$, are used. The likelihood function, $\mathcal{L}_{1}$ is

$$
\mathcal{L}_{1}=\mathcal{P}\left(\tilde{\mathrm{N}}_{\text {tight }}^{1 \mathrm{tag}}, \mathrm{N}_{\text {tight }}^{1 \mathrm{tag}}\right) \times \mathcal{P}\left(\tilde{\mathrm{N}}_{\text {loose-tight }}^{1 \text { tag }}, \mathrm{N}_{\text {loose-tight }}^{1 \text { tag }}\right)
$$

3. Equation 6.7 and 6.8 show the solution of the matrix method and the likelihood function in the two or more b-tag sample,

$$
\begin{gathered}
N_{W+t \bar{t}}^{2 \mathrm{tag}}=\varepsilon_{\text {sig }} \frac{\tilde{N}_{\text {tight }}^{2 \mathrm{tag}}-\varepsilon_{Q C D}\left(\tilde{N}_{\text {tight }}^{2 \mathrm{tag}}+\tilde{N}_{\text {loose-tight }}^{2 \mathrm{tag}}\right)}{\varepsilon_{\text {sig }}-\varepsilon_{Q C D}} \\
N_{Q C D}^{2 \mathrm{tag}}=\varepsilon_{Q C D} \frac{\varepsilon_{\text {sig }}\left(\tilde{N}_{\text {tight }}^{2 \mathrm{tag}}+\tilde{N}_{\text {loose-tight }}^{2 \mathrm{tag}}\right)-\tilde{N}_{\text {tight }}^{\text {tag }}}{\varepsilon_{\text {sig }}-\varepsilon_{Q C D}} \\
\mathcal{L}_{2}=\mathcal{P}\left(\tilde{\mathrm{N}}_{\text {tight }}^{2 \mathrm{tag}}, \mathrm{N}_{\text {tight }}^{2 \mathrm{tag}}\right) \times \mathcal{P}\left(\tilde{\mathrm{N}}_{\text {loose-tight }}^{2 \mathrm{tag}}, \mathrm{N}_{\text {loose-tight }}^{2 \operatorname{tag}}\right) .
\end{gathered}
$$

In the above likelihood functions, the number of events without a tilde is a floating parameter that is constrained to its observed value by including a Poisson probability term in the likelihood function.

The three subsamples (untagged, exactly one tag, and two or more tags) can be combined and 
the solutions of the matrix method for the number of overall events (before b-tagging) are given by

$$
\begin{aligned}
N_{W+t \bar{t}}= & N_{W+t \bar{t}}^{0 \text { tag }}+N_{W+t \bar{t}}^{1 \mathrm{tag}}+N_{W+t \bar{t}}^{2 \mathrm{tag}} \\
= & \frac{\varepsilon_{\text {sig }}}{\varepsilon_{\text {sig }}-\varepsilon_{Q C D}}\left(\left(N_{\text {tight }}^{0 \text { tag }}+N_{\text {tight }}^{1 \text { tag }}+N_{\text {tight }}^{2 \text { tag }}\right)-\varepsilon_{Q C D}\left(N_{\text {tight }}^{0 \text { tag }}+N_{\text {tight }}^{1 \text { tag }}+N_{\text {tight }}^{2 \text { tag }}\right)\right) \\
& -\frac{\varepsilon_{\text {sig }} \varepsilon_{Q C D}}{\varepsilon_{\text {sig }}-\varepsilon_{Q C D}}\left(N_{\text {loose-tight }}^{0 \text { tag }}+N_{\text {loose-tight }}^{1 \text { tag }}+N_{\text {loose-tight }}^{2 \text { tag }}\right) \\
N_{Q C D}= & N_{Q C D}^{0 \text { tag }}+N_{Q C D}^{1 \text { tag }}+N_{Q C D}^{2 \text { tag }} \\
= & \frac{\varepsilon_{Q C D}}{\varepsilon_{\text {sig }}-\varepsilon_{Q C D}}\left(\varepsilon_{\text {sig }}\left(N_{\text {tight }}^{\text {Otag }}+N_{\text {tight }}^{1 \text { tag }}+N_{\text {tight }}^{2 t a g}\right)-\left(N_{\text {tight }}^{\text {tatag }}+N_{\text {tight }}^{1 \text { tag }}+N_{\text {tight }}^{2 \text { tag }}\right)\right) \\
& +\frac{\varepsilon_{\text {sig }} \varepsilon_{Q C D}}{\varepsilon_{\text {sig }}-\varepsilon_{Q C D}}\left(N_{\text {loose-tight }}^{0 \text { tag }}+N_{\text {loose-tight }}^{1 \text { tag }}+N_{\text {loose-tight }}^{2 \text { tag }}\right)
\end{aligned}
$$

and the likelihood function can be expressed as follows

$$
\begin{aligned}
\mathcal{L}= & \mathcal{L}_{0} \times \mathcal{L}_{1} \times \mathcal{L}_{2} \\
= & \mathcal{P}\left(\tilde{N}_{\text {tight }}^{0 \text { tag }}, N_{\text {tight }}^{\text {Otag }}\right) \times \mathcal{P}\left(\tilde{N}_{\text {loose-tight }}^{\text {Otag }}, N_{\text {loose-tight }}^{\text {Otag }}\right) \\
& \times \mathcal{P}\left(\tilde{N}_{\text {tight }}^{1 \text { tag }}, N_{\text {tight }}^{1 \text { tag }}\right) \times \mathcal{P}\left(\tilde{N}_{\text {loose-tight }}^{1 \text { tag }}, N_{\text {loose-tight }}^{1 \text { tag }}\right) \\
& \times \mathcal{P}\left(\tilde{N}_{\text {tight }}^{2 \text { tag }}, N_{\text {tight }}^{2 \text { tag }}\right) \times \mathcal{P}\left(\tilde{N}_{\text {loose-tight }}^{2 \text { tag }}, N_{\text {loose-tight }}^{2 \text { tag }}\right) .
\end{aligned}
$$

Next, $N_{\text {tight }}^{1 \text { tag }}$ and $N_{\text {tight }}^{2 \text { tag }}$ are re-expressed in terms of $\sigma_{t \bar{t}}$, the number of background events predicted from MC, and the number of observed events in order to account for their dependence on $\sigma_{t \bar{t}}$ in the likelihood, with respect to $\sigma_{t \bar{t}}$

$$
\begin{aligned}
N_{t i g h t}^{1 t a g} & =P_{t \bar{t}}^{1 t a g} N_{t \bar{t}}+N_{Q C D}^{1 t a g}+P_{W}^{1 t a g} N_{W}+P_{M C b k g}^{1 t a g} N_{M C b k g} \\
N_{t i g h t}^{2 t a g} & =P_{t \bar{t}}^{2 t a g} N_{t \bar{t}}+N_{Q C D}^{2 t a g}+P_{W}^{2 t a g} N_{W}+P_{M C b k g}^{2 t a g} N_{M C b k g}
\end{aligned}
$$

where $P^{1 \text { tag }}\left(P^{2 \operatorname{tag}}\right)$ with subscriptions denotes the event probability calculated using Equation 5.16 (5.17). Equation 6.11 simply expresses that the number of tagged events is equal to the sum of number of tagged events from $t \bar{t}$, multijet, $\mathrm{W}+$ jets and other physics backgrounds estimated in 


\section{Chapter 5 .}

According to the Equation 5.9, the contribution of $\mathrm{W}+$ jets events in the before-tagging sample is

$$
N_{W}=N_{W+t \bar{t}}-N_{t \bar{t}}-N_{M C} b k g .
$$

After this is inserted into Equation 6.11, it is obtained that

$$
\begin{gathered}
N_{t i g h t}^{1 t a g}=P_{t \bar{t}}^{1 t a g} N_{t \bar{t}}+N_{Q C D}^{1 t a g}+P_{W}^{1 t a g} N_{W+t \bar{t}}-P_{W}^{1 t a g} N_{M C b k g}-P_{W}^{1 t a g} N_{t \bar{t}}+P_{M C b k g}^{1 t a g} N_{M C b k g} \\
N_{t i g h t}^{2 t a g}=P_{t \bar{t}}^{2 t a g} N_{t \bar{t}}+N_{Q C D}^{2 t a g}+P_{W}^{2 t a g} N_{W+t \bar{t}}-P_{W}^{2 t a g} N_{M C b k g}-P_{W}^{2 t a g} N_{t \bar{t}}+P_{M C b k g}^{2 t a g} N_{M C b k g} .
\end{gathered}
$$

In Equation 6.13, $N_{\text {tight }}^{1 t a g}$ and $N_{\text {tight }}^{2 t a g}$ appear on both sides of the equation, giving a system of two equations with two unknowns $\left(N_{\text {tight }}^{1 \text { tag }}\right.$ and $\left.N_{\text {tight }}^{2 t a g}\right)$. Since $N_{W+t \bar{t}}, N_{Q C D}^{1 t a g}$ and $N_{Q C D}^{2 t a g}$ can be expressed explicitly as a functions of $N_{\text {tight }}^{\text {0tag }}, N_{\text {tight }}^{1 \text { tag }}, N_{\text {tight }}^{2 \text { tag }}, N_{\text {loose-tight }}^{\text {Otag }}, N_{\text {loose-tight }}^{1 \text { tag }}$, and $N_{\text {loose-tight }}^{\text {2tag }}$ using Equations 6.5, 6.7 and 6.9, the system of equations can be rewritten as

$$
\begin{aligned}
N_{\text {tight }}^{1 \text { tag }}= & k_{1}+a_{1} N_{\text {tight }}^{0 \text { tag }}+b_{1} N_{\text {tight }}^{1 \text { tag }}+c_{1} N_{\text {tight }}^{2 \text { tag }} \\
& +d_{1} N_{\text {loose-tight }}^{0 \text { tag }}+e_{1} N_{\text {loose-tight }}^{1 \text { tag }}+f_{1} N_{\text {loose-tight }}^{2 \text { tag }} \\
N_{\text {tight }}^{2 \text { tag }}= & k_{2}+a_{2} N_{\text {tight }}^{0 \text { tag }}+b_{2} N_{\text {tight }}^{1 \text { tag }}+c_{2} N_{\text {tight }}^{2 \text { tag }} \\
& +d_{2} N_{\text {loose-tight }}^{\text {0tag }}+e_{2} N_{\text {loose-tight }}^{1 \text { tag }}+f_{2} N_{\text {loose-tight }}^{2 \text { tag }}
\end{aligned}
$$

where $k_{1}$ and $k_{2}$ are defined as

$$
\begin{aligned}
& k_{1}=P_{t \bar{t}}^{1 t a g} N_{t \bar{t}}-P_{W}^{1 t a g} N_{M C b k g}-P_{W}^{1 t a g} N_{t \bar{t}}+P_{M C b k g}^{1 t a g} N_{M C b k g} \\
& k_{2}=P_{t \bar{t}}^{2 t a g} N_{t \bar{t}}-P_{W}^{2 t a g} N_{M C b k g}-P_{W}^{2 t a g} N_{t \bar{t}}+P_{M C b k g}^{2 t a g} N_{M C b k g} .
\end{aligned}
$$

In Equation $6.14, a_{1}, b_{1}, c_{1}, d_{1}, e_{1}$, and $f_{1}$ are given by

$$
a_{1}=\frac{P_{W}^{1 t a g} \varepsilon_{s i g}\left(1-\varepsilon_{Q C D}\right)}{\varepsilon_{s i g}-\varepsilon_{Q C D}}
$$




$$
\begin{aligned}
b_{1} & =\frac{\varepsilon_{s i g} \varepsilon_{Q C D}-\varepsilon_{Q C D}+P_{W}^{1 t a g} \varepsilon_{s i g}-P_{W}^{1 t a g} \varepsilon_{s i g} \varepsilon_{Q C D}}{\varepsilon_{s i g}-\varepsilon_{Q C D}} \\
c_{1} & =a_{1} \\
d_{1} & =\frac{-P_{W}^{1 t a g} \varepsilon_{s i g} \varepsilon_{Q C D}}{\varepsilon_{s i g}-\varepsilon_{Q C D}} \\
e_{1} & =\frac{\varepsilon_{s i g} \varepsilon_{Q C D}-P_{W}^{1 t a g} \varepsilon_{s i g} \varepsilon_{Q C D}}{\varepsilon_{s i g}-\varepsilon_{Q C D}} \\
f_{1} & =d_{1}
\end{aligned}
$$

and $a_{2}, b_{2}, c_{2}, d_{2}, e_{2}, f_{2}$ are given by

$$
\begin{aligned}
& a_{2}=\frac{P_{W}^{2 t a g} \varepsilon_{s i g}\left(1-\varepsilon_{Q C D}\right)}{\varepsilon_{s i g}-\varepsilon_{Q C D}} \\
& b_{2}=a_{2} \\
& c_{2}=\frac{\varepsilon_{s i g} \varepsilon_{Q C D}-\varepsilon_{Q C D}+P_{W}^{2 t a g} \varepsilon_{s i g}-P_{W}^{2 t a g} \varepsilon_{s i g} \varepsilon_{Q C D}}{\varepsilon_{s i g}-\varepsilon_{Q C D}} \\
& d_{2}=\frac{-P_{W}^{2 t a g} \varepsilon_{s i g} \varepsilon_{Q C D}}{\varepsilon_{s i g}-\varepsilon_{Q C D}} \\
& e_{2}=d_{2} \\
& f_{2}=\frac{\varepsilon_{s i g} \varepsilon_{Q C D}-P_{W}^{2 t a g} \varepsilon_{s i g} \varepsilon_{Q C D}}{\varepsilon_{s i g}-\varepsilon_{Q C D}} .
\end{aligned}
$$

Solving Equation 6.14 for $N_{\text {tight }}^{1 t a g}$ and $N_{\text {tight }}^{2 t a g}$ gives

$$
\begin{aligned}
N_{\text {tight }}^{1 \text { tag }}= & \frac{1}{\left(c_{2}-1\right)\left(b_{1}-1\right)-c_{1} b_{2}} \times \\
& \left(k_{2} c_{1}-k_{1} c_{2}+k_{1}\right. \\
& +N_{\text {tight }}^{\text {Otag }}\left(c_{1} a_{2}-\left(c_{2}-1\right) a_{1}\right) \\
& +N_{\text {loose-tight }}^{\text {Otag }}\left(c_{1} d_{2}-\left(c_{2}-1\right) d_{1}\right) \\
& +N_{\text {loose-tight }}^{1 \text { tag }}\left(c_{1} e_{2}-\left(c_{2}-1\right) e_{1}\right) \\
& \left.+N_{\text {loose-tight }}^{2 \text { tag }}\left(c_{1} f_{2}-\left(c_{2}-1\right) f_{1}\right)\right) \\
N_{\text {tight }}^{2 \text { tag }}= & \frac{1}{\left(c_{2}-1\right)\left(b_{1}-1\right)-c_{1} b_{2}} \times
\end{aligned}
$$




$$
\begin{aligned}
& \left(k_{1} b_{2}-k_{2} b_{1}+k_{2}\right. \\
& +N_{\text {tight }}^{0 t a g}\left(b_{2} a_{1}-\left(b_{1}-1\right) a_{2}\right) \\
& +N_{\text {loose-tight }}^{0 t a g}\left(b_{2} d_{1}-\left(b_{1}-1\right) d_{2}\right) \\
& +N_{\text {loose-tight }}^{1 \text { tag }}\left(b_{2} e_{1}-\left(b_{1}-1\right) e_{2}\right) \\
& \left.+N_{\text {loose-tight }}^{2 \text { tag }}\left(b_{2} f_{1}-\left(b_{1}-1\right) f_{2}\right)\right) .
\end{aligned}
$$

Thus, $N_{\text {tight }}^{1 t a g}$ and $N_{\text {tight }}^{2 t a g}$ are expressed as a function of the tagging probabilities, efficiencies for the matrix method, the number of predicted events for the processes such as single top, diboson, $Z+j e t s$, the floating parameters $N_{\text {tight }}^{\text {0tag }}, N_{\text {loose-tight }}^{\text {0tag }}, N_{\text {loose-tight }}^{1 \text { tag }}$ and $N_{\text {loose-tight }}^{\text {2tag }}$ in Equation 6.18 and 6.19, and the expected number of $t \bar{t}$ events in the sample.

A single likelihood function is constructed by taking the product of the likelihoods $e+$ jets (3 jets), $e+$ jets ( 4 or more jets), $\mu+$ jets ( 3 jets), and $\mu+$ jets ( 4 or more jets) channels

$$
\begin{aligned}
\mathcal{L}= & \prod_{i} \mathcal{P}\left(\tilde{N}_{\text {tight }}^{0 t a g}, N_{\text {tight }}^{0 t a g}\right) \times \mathcal{P}\left(\tilde{N}_{\text {loose-tight }}^{\text {ttag }}, N_{\text {loose-tight }}^{\text {Otag }}\right) \\
& \times \mathcal{P}\left(\tilde{N}_{\text {tight }}^{1 t a g}, N_{\text {tight }}^{1 t a g}\right) \times \mathcal{P}\left(\tilde{N}_{\text {loose-tight }}^{1 \text { tag }}, N_{\text {loose-tight }}^{1 \text { tag }}\right) \\
& \times \mathcal{P}\left(\tilde{N}_{\text {tight }}^{\text {ttag }}, N_{\text {tight }}^{2 t a g}\right) \times \mathcal{P}\left(\tilde{N}_{\text {loose-tight }}^{2 \text { tag }}, N_{\text {loose-tight }}^{\text {ttag }}\right)
\end{aligned}
$$

where $i$ denotes the channel. $\mathcal{L}$ summarizes all Poisson constraints used in the cross section calculation. The top quark pair production cross section, $\sigma_{t \bar{t}}$ is determined by maximizing the likelihood $\mathcal{L}$ by varying the parameters $N_{\text {loose-tight }}^{0 \text { tag }}, N_{\text {loose-tight }}^{1 \text { tag }}, N_{\text {loose-tight }}^{2 \text { tag }}, N_{\text {tight }}^{0 \text { tag }}, N_{\text {tight }}^{1 \text { tag }}$, and $N_{\text {tight }}^{2 \text { tag }} . N_{\text {tight }}^{1 \text { tag }}$ and $N_{\text {tight }}^{2 t a g}$ are the functions of $\sigma_{t \bar{t}}$.

\subsection{Cross Section with Statistical Uncertainty}

The results of the cross section calculation are presented in this section for exactly one tag events and two or more tags events using the procedure discussed in the previous section. Table 6.1 shows the cross sections measured for each channel and each sample. Combined results are shown in 
Table 6.2. The errors in the tables are statistical only. The statistical error is determined by the width bewteen two points that change one unit from the maximized likelihood at the parabolic curve of the likelihood.

\begin{tabular}{|c|c|c|c|c|}
\hline \hline \multirow{2}{*}{ Channel } & \multicolumn{2}{|c|}{$e+$ jets $(\mathrm{pb})$} & \multicolumn{2}{c|}{$\mu+$ jets $(\mathrm{pb})$} \\
\cline { 2 - 5 } & 3 jets & 4 or more jets & 3 jets & 4 or more jets \\
\hline \multirow{2}{*}{ Exactly One Tagged Events } & $7.48_{-1.38}^{+1.45}$ & $8.05_{-1.17}^{+1.25}$ & $7.12_{-1.65}^{+1.74}$ & $11.05_{-1.48}^{+1.58}$ \\
\cline { 2 - 6 } & \multicolumn{2}{|c|}{$7.81_{-0.90}^{+0.94}$} & \multicolumn{2}{|c|}{$9.48_{-1.09}^{+1.14}$} \\
\hline \multirow{2}{*}{ Two or More Tagged Events } & $8.05_{-1.67}^{+1.84}$ & $5.39_{-1.18}^{+1.33}$ & $8.60_{-1.96}^{+2.18}$ & $8.22_{-1.53}^{+1.72}$ \\
\cline { 2 - 5 } & \multicolumn{2}{|c|}{$6.47_{-1.01}^{+1.10}$} & \multicolumn{2}{|c|}{$8.37_{-1.23}^{+1.33^{2}}$} \\
\hline \hline
\end{tabular}

Table 6.1: The $t \bar{t}$ cross section results for each channel. The errors are statistical only.

\begin{tabular}{|l|c|c|}
\hline \hline & $e+$ jets $(\mathrm{pb})$ & $\mu+$ jets $(\mathrm{pb})$ \\
\hline \multirow{3}{*}{ Combined Cross Section } & $7.27_{-0.69}^{+0.72}$ & $9.03_{-0.83}^{+0.87}$ \\
\cline { 2 - 3 } & \multicolumn{2}{|c|}{$8.05_{-0.53}^{+0.55}$} \\
\hline \hline
\end{tabular}

Table 6.2: The combined results of cross section. The errors are statistical only.

Figures $6.1 \sim 6.3$ show the signal and background compositions that result from these fits. The combined cross section result of $\sigma_{t \bar{t}}=8.05 \mathrm{pb}$ is used for these figures.

\subsection{Systematic Uncertainties}

The systematic uncertainties from different sources can effect the preselection efficiencies and btagging probabilities. In this section, the various sources of systematic uncertainties and their results are discussed.

The biggest systematic uncertainty is resulted from uncertainty on the integrated luminosity measurement and it is $6.1 \%$ [49]. It is assigned as additional systematic uncertainty on the measured cross section.

A complete list of systematic uncertainties is shown in Table 6.3, where a cross denotes that the uncertainty is applicable to the channel. Uncertainties whose origin is statistical are treated as being 

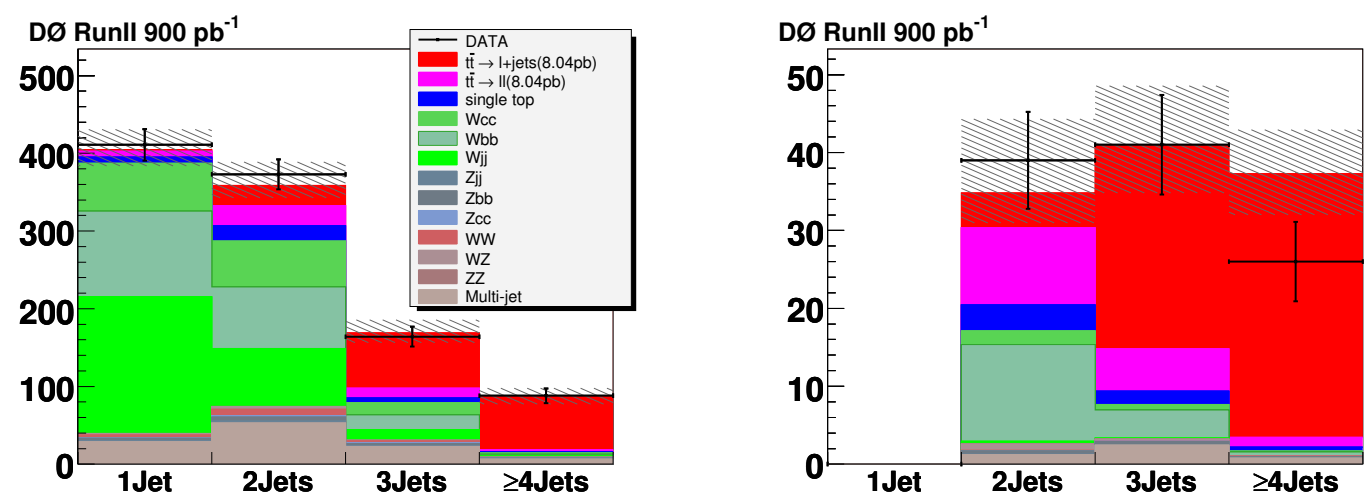

Figure 6.1: The predicted signal and background compositions for exactly one tag (left) and two or more tags (right) samples in the $e+$ jets channel with the measured $t \bar{t}$ cross section. The combined cross section result for all channels $(8.05 \mathrm{pb})$ is used in the figure. The red area is the contribution of $t \bar{t} \rightarrow l+$ jets signal events and the shaded region in the plots indicates the statistical uncertainty in the predicted number of events.
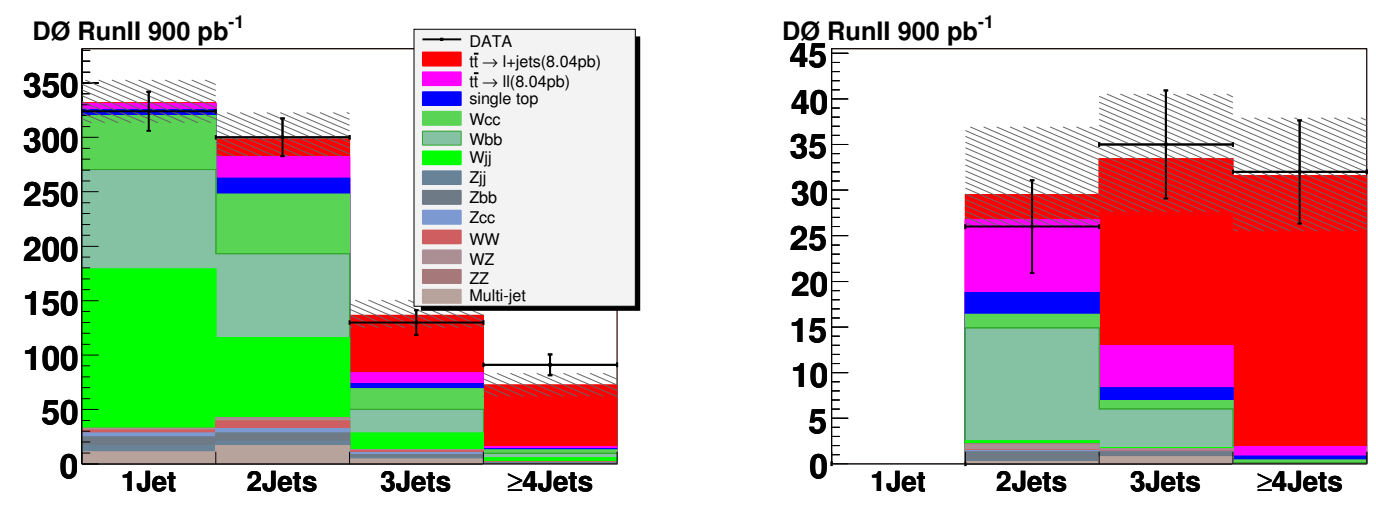

Figure 6.2: The predicted signal and background compositions for exactly one tag (left) and two or more tags (right) samples in the $\mu+$ jets channel with the measured $t \bar{t}$ cross section. The combined cross section result for all channels $(8.05 \mathrm{pb})$ is used in the figure. The red area is the contribution of $t \bar{t} \rightarrow l+$ jets signal events and the shaded region in the plots indicates the statistical uncertainty in the predicted number of events. 

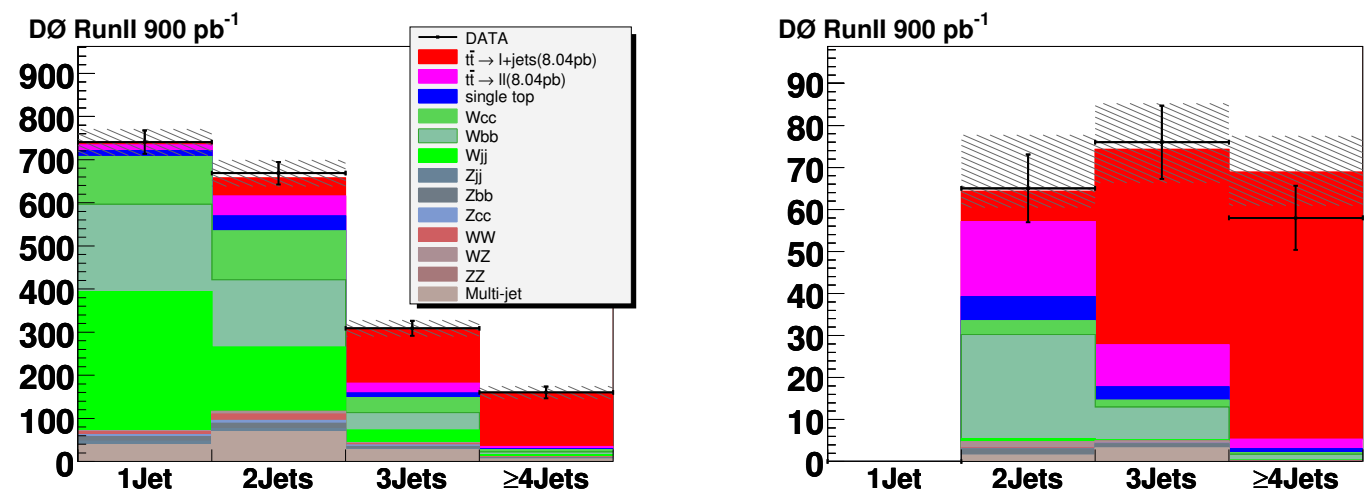

Figure 6.3: The predicted signal and background compositions for exactly one tag (left) and two or more tags (right) samples in the lepton+jets channel with the measured $t \bar{t}$ cross section. The combined cross section result for all channels $(8.05 \mathrm{pb})$ is used in the figure. The red area is the contribution of $t \bar{t} \rightarrow l+$ jets signal events and the shaded region in the plots indicates the statistical uncertainty in the predicted number of events.

uncorrelated, and while non-statistical uncertainties are related as being fully correlated between the $e+$ jets and $\mu+$ jets channels.

\subsubsection{Uncertainties on the Preselection Efficiency}

The systematic uncertainties contributing to the preselection efficiency are briefly described in this section.

Primary Vertex scale factor This systematic uncertainty arises from the difference between data and $\mathrm{MC}$ for the selection of primary vertices, where $|Z|<60 \mathrm{~cm}, \geq 3$ tracks and $\Delta \mid P V-$ $P V_{\text {Lepton }} \mid<1 \mathrm{~cm}$ are required. This uncertainty is estimated to be $1.5 \%$ in Reference [56].

Vertex $Z$ position simulation This uncertainty is based on Reference [63]. The uncertainty is from the inaccuracies in the vertex $\mathrm{z}$ position simulation. It is measured to be $1.6 \%$ for the data set used in this analysis.

Luminosity profile in data and MC There is a difference between MC and data luminosity profiles and it is necessary to reweight the MC to match the luminosity profile in the data. This correction is not added to the central value, instead it is treated as a systematic uncertainty 
in this analysis.

$\mathbf{Z} p_{\mathrm{T}}$ reweighting The uncertainty due to $\mathrm{Z} p_{\mathrm{T}}$ reweighting in the Monte Carlo to match data was taken into account using the constant value of $12 \%$ uncertainty quoted in [55].

Electron identification efficiency According to studies by the dilepton cross section measurement group at $\mathrm{D} \varnothing$, this systematic uncertainty is $2.5 \% .1 .3 \%$ is from a jet multiplicity dependence [60] in the electron ID efficiency and 2.3\% [64] from dependence on other quantities.

Muon identification efficiency This systematic uncertainty is estimated by the muon ID group to be $0.7 \%$, and is due to the uncertainty in the muon reconstruction efficiency [24].

Muon tracking efficiency This systematic uncertainty is also estimated by the muon ID group to be $0.7 \%$, and is due to the uncertainty in the efficiency for matching tracks reconstructed in the central tracker and the local muon track reconstructed by the muon detector [24].

Muon isolation efficiency This is the uncertainty in the isolation scale factor for muons and is found to be $2 \%[24]$.

Trigger efficiency The trigger efficiency as a function of $p_{\mathrm{T}}$ for each object in MC event is shifted up and down by one standard deviation and then the weight of the event is recalculated. This shift is taken as an overall constant systematic error on the MC acceptance.

Parton distribution functions The computed acceptance for $t \bar{t}$ events depends on the parton distribution functions used. It is an uncertainty that corresponds to the shift observed when the parton momenta are reweighted from the CTEQ6L1 pdf set to the CTEQ6.1M sets [60].

Cross section for MC background estimation The errors on the NLO cross sections used in the single top, Z+jets, and diboson samples, are $12.6 \%$ [53], 15\% [54], and $20 \%$ [56] respectively. These NLO cross sections are shifted up and down by those errors and the $t \bar{t}$ cross section is recalculated. This uncertainty is determined by the difference between the central value and recalculation. 
Signal Modeling In this analysis, the $t \bar{t}$ MC sample is generated using PYTHIA. To determine the generator dependence, the $t \bar{t}$ MC sample generated with PYTHIA is substituted by a sample generated with ALPGEN. Then, the relative error in the signal preselection efficiency between the two different $t \bar{t}$ MC simulations is calculated.

\subsubsection{Uncertainties on Both Preselection Efficiency and Tagging Proba- bility}

In this section, the sources of systematic uncertainties contributing to the preselection efficiency as well as the b-tagging probability are described.

JES Uncertainty The effect of the jet energy scale (JES) uncertainty [29] is obtained by varying the JES by $\pm 1 \sigma$, where

$$
\sigma=\sqrt{\sigma_{\text {stat }, \text { data }}^{2}+\sigma_{\text {syst }, \text { data }}^{2}+\sigma_{\text {stat }, M C}^{2}+\sigma_{\text {syst }, M C}^{2}}
$$

Jet energy resolution (JSSR) The uncertainty on the jet energy resolution in data is already included in the systematic uncertainty due to the jet energy scale. The energy of jets in Monte Carlo is smeared to the jet energy resolution of the data. The jet energy resolution in Monte Carlo has an uncertainty which is not taken into account in the jet energy scale. To account for this effect, the parameters of the jet energy smearing are varied by the size of the uncertainty in the jet energy resolution parameters in Monte Carlo.

Jet Reconstruction and Identification Efficiency (JetID) This is the systematic uncertainty on the correction of the jet reconstruction $\times$ identification efficiency in the simulation.

$b$ fragmentation The $\mathrm{D} \emptyset \mathrm{MC}$ simulation uses the default $b$-quark fragmentation function provided by PYTHIA. The difference between this default fragmentation function and the fragmentation function tuned to LEP data [65] is quoted as a systematic uncertainty. The LEP fragmentation function was implemented by reweighting the events accordingly. 
Tag Rate Functions (TRF) The b-ID group provide the b-ID package and the errors for taggability and TRF are summed quadratically. The uncertainty associated with the TRF is evaluated by raising and lowering the error by one standard deviation in order to determine the new event tagging weight. The TRF uncertainties originate from several sources: statistical errors in MC data sets, the assumed fraction of heavy flavor in the MC QCD sample used for the mistag rate determination, and the parameterizations of the tag rate functions.

Flavor dependence of taggability (FDT) This uncertainty is obtained by substituting the parameterized taggability for the QCD MC sample by the taggability for the $W b \bar{b}$ and $W c \bar{c} \mathrm{MC}$ samples for the b-jets and c-jets, respectively. The result, which was studied in p14 analysis [66], is used in this analysis.

W TRF correction At low jet $p_{\mathrm{T}}$, there is a discrepancy in the $W b \bar{b}$ and $W c \bar{c} \mathrm{MC}$ samples between the actual tagging efficiency and the TRF because of the low b-quark momentum in these samples [67]. The correction factor is measured and the effect of this factor is treated as a systematic uncertainty.

MC Factorization scale The factorization scale is estimated by calculating the fitted number of $t \bar{t}$ events in the $W+$ jets background Monte Carlo sample generated with $Q^{2}=<p_{T_{j}}>^{2}$ instead of the default scale $Q^{2}=M_{W}^{2}+\sum p_{T_{j}}^{2}$. Changing the factorization scale from the default $Q^{2}=$ $M_{W}^{2}+\sum p_{T_{j}}^{2}$ to $Q^{2}=<p_{T_{j}}>^{2}$ leads to a softer $p_{T}$ spectra, whereas other tested factorization scales lead to a harder $p_{T}$ spectra. Since the distributions for $Q^{2}=M_{W}^{2}+\sum p_{T_{j}}^{2}$ are found to be well centered between these other choices, it is a good approximation to symmetrize the one-sided error determined from the choice of $Q^{2}=<p_{T_{j}}>^{2}$ as the factorization scale [68].

Heavy flavor (HF) scale factor This systematic uncertainty comes from the heavy flavor scale factor between $W j j$ and $W b b / W c c$ samples, which is $1.17 \pm 0.18$. For $\mathrm{Z}+\mathrm{jets}, 1.35$ is used as the heavy flavor scale factor with $50 \%$ uncertainty.

Limited MC statistics This uncertainty results from limited MC statistics. The statistical uncertainties on the determined preselection efficiencies in $\mathrm{MC}$ samples are used to calculate this 
uncertainty.

Uncertainty on $\varepsilon_{s i g}$ and $\varepsilon_{Q C D}$ The uncertainty in the number of $W+$ jets and QCD events per exclusive jet multiplicity is obtained by varying $\varepsilon_{s i g}$ and $\varepsilon_{Q C D}$ by one standard deviation. The results of the two variations are summed in quadrature. The uncertainties are assumed to be totally uncorrelated between the $e+$ jets and $\mu+$ jets channels.

\subsubsection{Summary of Systematic Uncertainties}

Summaries of systematic uncertainties are shown in Tables $6.4 \sim 6.6$. The systematic uncertainty on the cross section is calculated for each independent source by making a one standard deviation variation up and down and then propagating the variation into both the fitted number of $t \bar{t}$ events and the signal efficiencies.

\subsection{Results}

The final results of the top quark pair production cross section measurements are presented in this section. The results from the combined cross sections for exactly one b-tag and two or more b-tags in the lepton + jets channel are

$$
\begin{gathered}
\ell+\text { jets }(=1 \mathrm{tag}) \quad: \quad \sigma_{p \bar{p} \rightarrow t \bar{t}+X}=8.53_{-0.70}^{+0.72} \text { (stat) }{ }_{-0.54}^{+0.58} \text { (syst) } \pm 0.52 \text { (lum) pb } \\
\ell+\text { jets }(\geq 2 \text { tags }): \quad \sigma_{p \bar{p} \rightarrow t \bar{t}+X}=7.33_{-0.79}^{+0.84} \text { (stat) }{ }_{-0.89}^{+1.01} \text { (syst) } \pm 0.45 \text { (lum) pb }
\end{gathered}
$$

and the final combined result for all channels is

$$
\ell+\text { jets }: \quad \sigma_{p \bar{p} \rightarrow t \bar{t}+X}=8.05_{-0.53}^{+0.55} \text { (stat) }{ }_{-0.67}^{+0.72} \text { (syst) } \pm 0.49 \text { (lum) pb }
$$

The dependence of the cross section on the top quark mass is shown in Figure 6.4. It is fitted with 
a second order polynomial and the result is $\sigma_{t \bar{t}}=0.001090 \cdot M_{t o p}{ }^{2}-0.457093 \cdot M_{t o p}+54.610092$. In the figure, the result measured in this analysis is compared to two different theoretical cross sections by Cacciari et al. $\left(\sigma=6.70+0.71-0.88 \mathrm{pb}\right.$ at $\left.M_{t o p}=175 \mathrm{GeV}\right)[8]$ and Kidonakis and Vogt $\left(6.77 \pm 0.42 \mathrm{pb}\right.$ at $\left.M_{\text {top }}=175 \mathrm{GeV}\right)[7]$. The green (blue) dotted lines indicates the uncertainties in the cross section predictions (the measurement in this analysis).

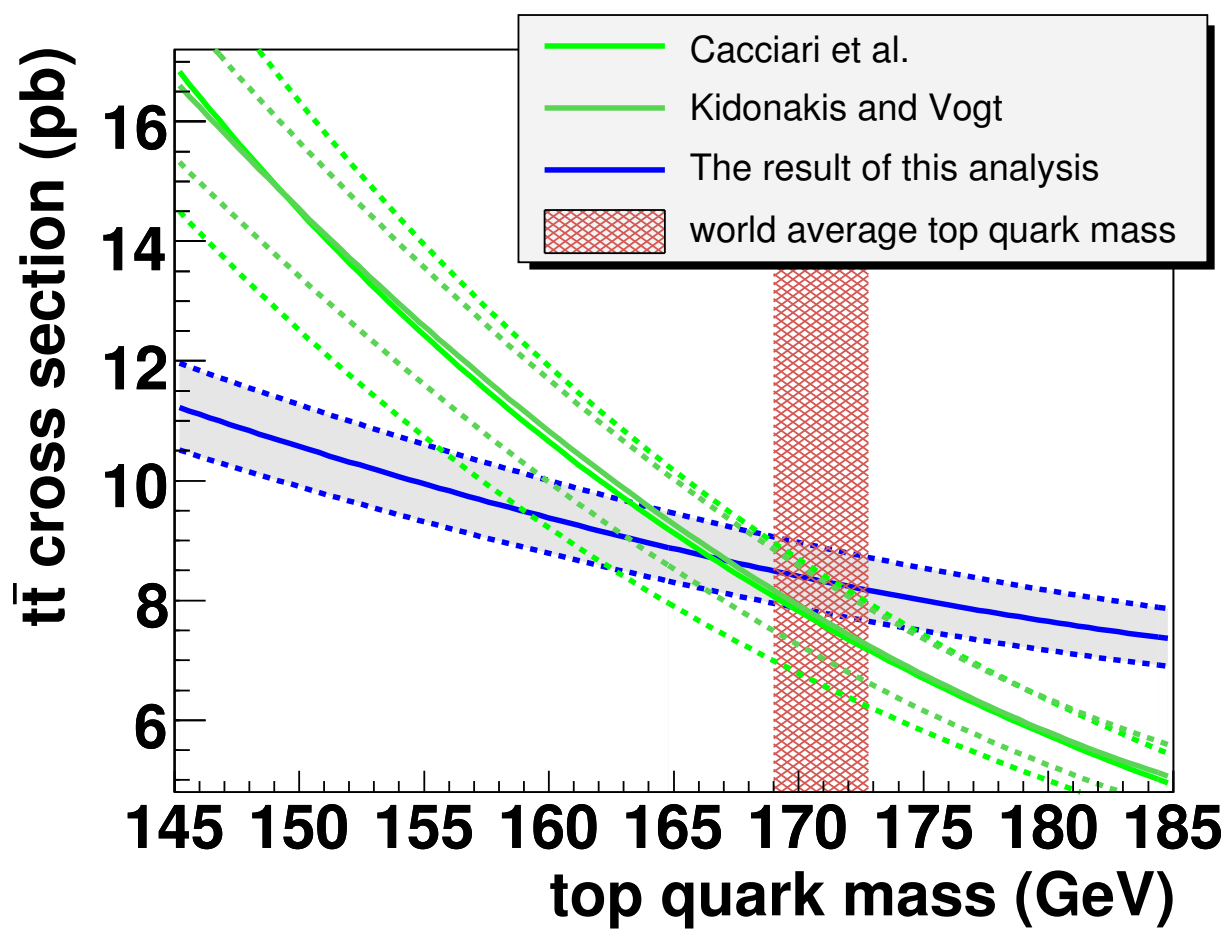

Figure 6.4: The measured top quark pair production cross section as a function of the top quark mass. The two green lines are the theoretical cross sections calculated by Cacciari et al. and Kidonakis and Vogt. The green (blue) dotted lines denote the uncertainties in the cross section The world average top quark mass at present, predictions (the measurement in this analysis). $172.6 \pm 0.8$ (stat) \pm 1.1 (sys) [11], is also shown in this figure. 


\begin{tabular}{|c|c|c|}
\hline Channel & $e+$ jets & $\mu+$ jets \\
\hline \multicolumn{3}{|l|}{ Correlated } \\
\hline Primary Vertex & $\times$ & $\times$ \\
\hline Luminosity Reweighting & $x$ & $x$ \\
\hline $\mathrm{Z} p_{\mathrm{T}}$ Reweighting & $x$ & $x$ \\
\hline Jet Trigger & $\times$ & $\times$ \\
\hline $\mathrm{PDF}$ & $x$ & $x$ \\
\hline Signal Modeling & $x$ & $x$ \\
\hline MC cross section & $\times$ & $\times$ \\
\hline JES & $x$ & $\times$ \\
\hline JSSR & $x$ & $\times$ \\
\hline Jet Identification & $\times$ & $x$ \\
\hline b-fragmentation & $x$ & $x$ \\
\hline Factorization & $\times$ & $\times$ \\
\hline TRF & $x$ & $x$ \\
\hline Flavor Dependence of taggability & $\times$ & $\times$ \\
\hline W TRF correction & $x$ & $\times$ \\
\hline W Heavy Flavor Scale Factor & $x$ & $\times$ \\
\hline Z Heavy Flavor Scale Factor & $\times$ & $\times$ \\
\hline \multicolumn{3}{|l|}{ Uncorrelated } \\
\hline EM ID & $x$ & \\
\hline EM Trigger & $x$ & \\
\hline Muon ID & & $\times$ \\
\hline Muon Tracking & & $\times$ \\
\hline Muon Isolation & & $\times$ \\
\hline$\mu$ Trigger & & $\times$ \\
\hline MC statistics & $\times$ & $\times$ \\
\hline$\varepsilon_{\text {sig }}$ & $\times$ & $\times$ \\
\hline$\varepsilon_{Q C D}$ & $\times$ & $\times$ \\
\hline
\end{tabular}

Table 6.3: Summary of the systematic uncertainties. The notation "uncorrelated" and "correlated" refer to the treatment of the sources of errors in the cross section combination. 


\begin{tabular}{|c|c|c|c|c|c|c|c|}
\hline & & \multicolumn{2}{|c|}{$e+j e t s$} & \multicolumn{2}{|c|}{$\mu+$ jets } & \multicolumn{2}{|c|}{$l+$ jets } \\
\hline type & source & $\overline{\sigma^{+}}$ & $\overline{\sigma^{-}}$ & " & $\bar{c}^{-}$ & " & 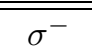 \\
\hline \multirow{3}{*}{ preselection } & Luminosity Reweighting & +0.02 & -0.00 & +0.15 & -0.00 & +0.07 & -0.00 \\
\hline & Primary Vertex & +0.14 & -0.13 & +0.17 & -0.16 & +0.15 & -0.15 \\
\hline & $Z p_{\mathrm{T}}$ Reweighting & +0.00 & -0.00 & +0.00 & -0.00 & +0.00 & -0.00 \\
\hline \multirow{7}{*}{$e+j e t s$ only } & $E M I D$ & +0.18 & -0.18 & - & - & +0.12 & -0.11 \\
\hline & L1EMtrigger & +0.08 & -0.01 & - & - & +0.05 & -0.01 \\
\hline & L2EMtrigger & +0.00 & -0.00 & - & - & +0.00 & -0.00 \\
\hline & L3EMtrigger & +0.05 & -0.05 & - & - & +0.03 & -0.03 \\
\hline & L1JetTrigger & +0.00 & -0.00 & - & - & +0.00 & -0.00 \\
\hline & L2JetTrigger & +0.00 & -0.00 & - & - & +0.00 & -0.00 \\
\hline & L3JetTrigger & +0.00 & -0.00 & - & - & +0.00 & -0.00 \\
\hline \multirow{4}{*}{$\mu+$ jets only } & Muon ID & - & - & +0.07 & -0.07 & +0.03 & -0.03 \\
\hline & Muon Tracking & - & - & +0.07 & -0.07 & +0.03 & -0.03 \\
\hline & Muon Isolation & - & - & +0.20 & -0.19 & +0.07 & -0.07 \\
\hline & Muon Trigger & - & - & +0.27 & -0.24 & +0.10 & -0.09 \\
\hline \multirow{4}{*}{ Jets } & Jet Trigger & +0.02 & -0.02 & +0.02 & -0.02 & +0.02 & -0.02 \\
\hline & $J E S$ & +0.30 & -0.25 & +0.30 & -0.23 & +0.30 & -0.24 \\
\hline & $J S S R$ & +0.00 & -0.09 & +0.00 & -0.08 & +0.00 & -0.08 \\
\hline & Jet Identification & +0.12 & -0.12 & +0.11 & -0.11 & +0.12 & -0.12 \\
\hline \multirow{2}{*}{ Matrix Method } & $\varepsilon_{s i g}$ & +0.03 & -0.03 & +0.08 & -0.07 & +0.05 & -0.05 \\
\hline & $\varepsilon_{Q C D}$ & +0.02 & -0.02 & +0.01 & -0.01 & +0.03 & -0.03 \\
\hline \multirow{3}{*}{ MC Modeling } & W HF Scale Factor & +0.20 & -0.19 & +0.28 & -0.26 & +0.24 & -0.22 \\
\hline & b-fragmetation & +0.18 & -0.00 & +0.18 & -0.00 & +0.19 & -0.00 \\
\hline & Factorization & +0.04 & -0.03 & +0.06 & -0.04 & +0.05 & -0.03 \\
\hline \multirow{5}{*}{ b-tagging } & $F D T$ & +0.00 & -0.13 & +0.00 & -0.00 & +0.00 & -0.07 \\
\hline & $W T R F$ correction & +0.07 & -0.00 & +0.10 & -0.00 & +0.09 & -0.00 \\
\hline & b-jets TRF & +0.20 & -0.15 & +0.21 & -0.16 & +0.21 & -0.16 \\
\hline & $c$-jets TRF & +0.06 & -0.06 & +0.08 & -0.08 & +0.07 & -0.07 \\
\hline & light-jets TRF & +0.06 & -0.06 & +0.08 & -0.08 & +0.07 & -0.07 \\
\hline \multirow{6}{*}{ Others } & Z HF Scale Factor & +0.09 & -0.09 & +0.12 & -0.12 & +0.10 & -0.10 \\
\hline & Signal Modeling & +0.00 & -0.23 & +0.00 & -0.23 & +0.00 & -0.23 \\
\hline & $M C$ cross section & +0.04 & -0.04 & +0.04 & -0.04 & +0.04 & -0.04 \\
\hline & $M C$ statistics on signal & +0.01 & -0.01 & +0.01 & -0.01 & +0.01 & -0.01 \\
\hline & MC statistics on background & +0.04 & -0.05 & +0.05 & -0.06 & +0.05 & -0.05 \\
\hline & $P D F$ & +0.02 & -0.02 & +0.02 & -0.02 & +0.02 & -0.02 \\
\hline \multicolumn{2}{|r|}{ Total } & +0.56 & -0.54 & +0.69 & -0.62 & +0.58 & -0.54 \\
\hline
\end{tabular}

Table 6.4: Summary of the total systematic uncertainties for the combined results (exactly one b-tag sample). 


\begin{tabular}{|c|c|c|c|c|c|c|c|}
\hline & & \multicolumn{2}{|c|}{$e+j e t s$} & \multicolumn{2}{|c|}{$\mu+$ jets } & \multicolumn{2}{|c|}{$l+$ jets } \\
\hline type & source & $\sigma^{+}$ & $\sigma^{-}$ & $\sigma^{+}$ & $\sigma^{-}$ & $\sigma^{+}$ & $\sigma^{-}$ \\
\hline \multirow{3}{*}{ preselection } & Luminosity Reweighting & +0.02 & -0.00 & +0.11 & -0.00 & +0.06 & -0.00 \\
\hline & Primary Vertex & +0.12 & -0.11 & +0.15 & -0.14 & +0.13 & -0.13 \\
\hline & $Z p_{\mathrm{T}}$ Reweighting & +0.00 & -0.00 & +0.00 & -0.00 & +0.00 & -0.00 \\
\hline \multirow{7}{*}{$e+j e t s$ only } & $E M I D$ & +0.15 & -0.15 & - & - & +0.09 & -0.09 \\
\hline & L1EMtrigger & +0.07 & -0.01 & - & - & +0.04 & -0.01 \\
\hline & L2EMtrigger & +0.00 & -0.00 & - & - & +0.00 & -0.00 \\
\hline & L3EMtrigger & +0.04 & -0.04 & - & - & +0.02 & -0.02 \\
\hline & L1JetTrigger & +0.00 & -0.00 & - & - & +0.00 & -0.00 \\
\hline & L2JetTrigger & +0.00 & -0.00 & - & - & +0.00 & -0.00 \\
\hline & L3JetTrigger & +0.00 & -0.00 & - & - & +0.00 & -0.00 \\
\hline \multirow{4}{*}{$\mu+$ jets only } & Muon ID & - & - & +0.06 & -0.06 & +0.02 & -0.02 \\
\hline & Muon Tracking & - & - & +0.06 & -0.06 & +0.02 & -0.02 \\
\hline & Muon Isolation & - & - & +0.18 & -0.17 & +0.07 & -0.07 \\
\hline & Muon Trigger & - & - & +0.24 & -0.21 & +0.09 & -0.08 \\
\hline \multirow{4}{*}{ Jets } & Jet Trigger & +0.01 & -0.01 & +0.02 & -0.02 & +0.02 & -0.02 \\
\hline & $J E S$ & +0.32 & -0.27 & +0.36 & -0.30 & +0.34 & -0.28 \\
\hline & $J S S R$ & +0.00 & -0.09 & +0.00 & -0.10 & +0.00 & -0.10 \\
\hline & Jet Identification & +0.13 & -0.13 & +0.12 & -0.12 & +0.13 & -0.13 \\
\hline \multirow{2}{*}{ Matrix Method } & $\varepsilon_{s i g}$ & +0.03 & -0.03 & +0.08 & -0.07 & +0.05 & -0.05 \\
\hline & $\varepsilon_{Q C D}$ & +0.02 & -0.02 & +0.05 & -0.04 & +0.00 & -0.01 \\
\hline \multirow{3}{*}{ MC Modeling } & W HF Scale Factor & +0.08 & -0.08 & +0.10 & -0.10 & +0.09 & -0.09 \\
\hline & b-fragmetation & +0.20 & -0.00 & +0.19 & -0.00 & +0.20 & -0.00 \\
\hline & Factorization & +0.02 & -0.02 & +0.03 & -0.02 & +0.03 & -0.02 \\
\hline \multirow{5}{*}{ b-tagging } & $F D T$ & +0.00 & -0.01 & +0.00 & -0.03 & +0.00 & -0.02 \\
\hline & $W T R F$ correction & +0.04 & -0.00 & +0.05 & -0.00 & +0.05 & -0.00 \\
\hline & b-jets TRF & +0.80 & -0.69 & +0.99 & -0.86 & +0.89 & -0.76 \\
\hline & $c$-jets TRF & +0.06 & -0.06 & +0.07 & -0.07 & +0.07 & -0.07 \\
\hline & light-jets TRF & +0.02 & -0.02 & +0.03 & -0.03 & +0.02 & -0.02 \\
\hline \multirow{6}{*}{ Others } & Z HF Scale Factor & +0.04 & -0.04 & +0.05 & -0.05 & +0.04 & -0.04 \\
\hline & Signal Modeling & +0.00 & -0.22 & +0.00 & -0.23 & +0.00 & -0.22 \\
\hline & $M C$ cross section & +0.03 & -0.03 & +0.03 & -0.03 & +0.03 & -0.03 \\
\hline & $M C$ statistics on signal & +0.01 & -0.01 & +0.01 & -0.01 & +0.01 & -0.01 \\
\hline & MC statistics on background & +0.01 & -0.01 & +0.01 & -0.02 & +0.01 & -0.01 \\
\hline & $P D F$ & +0.02 & -0.02 & +0.02 & -0.02 & +0.02 & -0.02 \\
\hline \multicolumn{2}{|r|}{ Total } & +0.92 & -0.82 & +1.15 & -1.01 & +1.01 & -0.89 \\
\hline
\end{tabular}

Table 6.5: Summary of the total systematic uncertainties for the combined results (two or more b-tags sample). 


\begin{tabular}{|c|c|c|c|c|c|c|c|}
\hline & & \multicolumn{2}{|c|}{$e+j e t s$} & \multicolumn{2}{|c|}{$\mu+$ jets } & \multicolumn{2}{|c|}{$l+j e t s$} \\
\hline type & source & $\sigma^{+}$ & $\sigma^{-}$ & $\sigma^{+}$ & $\sigma^{-}$ & $\bar{\sigma} \sigma^{+}$ & $\sigma^{-}$ \\
\hline \multirow{3}{*}{ preselection } & Luminosity Reweighting & +0.02 & -0.00 & +0.13 & -0.00 & +0.07 & -0.00 \\
\hline & Primary Vertex & +0.13 & -0.13 & +0.16 & -0.15 & +0.14 & -0.14 \\
\hline & $Z p_{\mathrm{T}}$ Reweighting & +0.00 & -0.00 & +0.00 & -0.00 & +0.00 & -0.00 \\
\hline \multirow{7}{*}{$e+j e t s$ only } & $E M I D$ & +0.17 & -0.16 & - & - & +0.11 & -0.11 \\
\hline & L1EMtrigger & +0.08 & -0.01 & - & - & +0.05 & -0.01 \\
\hline & L2EMtrigger & +0.00 & -0.00 & - & - & +0.00 & -0.00 \\
\hline & L3EMtrigger & +0.04 & -0.04 & - & - & +0.03 & -0.03 \\
\hline & L1JetTrigger & +0.00 & -0.00 & - & - & +0.00 & -0.00 \\
\hline & L2JetTrigger & +0.00 & -0.00 & - & - & +0.00 & -0.00 \\
\hline & L3JetTrigger & +0.00 & -0.00 & - & - & +0.00 & -0.00 \\
\hline \multirow{4}{*}{$\mu+j e t s$ only } & Muon ID & - & - & +0.07 & -0.06 & +0.02 & -0.02 \\
\hline & Muon Tracking & - & - & +0.07 & -0.06 & +0.02 & -0.02 \\
\hline & Muon Isolation & - & - & +0.19 & -0.18 & +0.07 & -0.07 \\
\hline & Muon Trigger & - & - & +0.26 & -0.23 & +0.09 & -0.09 \\
\hline \multirow{4}{*}{ Jets } & Jet Trigger & +0.02 & -0.02 & +0.02 & -0.02 & +0.02 & -0.02 \\
\hline & $J E S$ & +0.31 & -0.26 & +0.32 & -0.25 & +0.32 & -0.26 \\
\hline & $J S S R$ & +0.00 & -0.09 & +0.00 & -0.09 & +0.00 & -0.09 \\
\hline & Jet Identification & +0.12 & -0.12 & +0.12 & -0.12 & +0.12 & -0.12 \\
\hline \multirow{2}{*}{ Matrix Method } & $\varepsilon_{s i g}$ & +0.03 & -0.03 & +0.08 & -0.07 & +0.05 & -0.05 \\
\hline & $\varepsilon_{Q C D}$ & +0.03 & -0.03 & +0.02 & -0.02 & +0.02 & -0.03 \\
\hline \multirow{3}{*}{ MC Modeling } & W HF Scale Factor & +0.16 & -0.15 & +0.21 & -0.19 & +0.18 & -0.17 \\
\hline & b-fragmetation & +0.19 & -0.00 & +0.19 & -0.00 & +0.19 & -0.00 \\
\hline & Factorization & +0.03 & -0.03 & +0.05 & -0.03 & +0.04 & -0.03 \\
\hline \multirow{5}{*}{$b$-tagging } & $F D T$ & +0.00 & -0.08 & +0.00 & -0.01 & +0.00 & -0.05 \\
\hline & $W T R F$ correction & +0.06 & -0.00 & +0.08 & -0.00 & +0.07 & -0.00 \\
\hline & b-jets TRF & +0.46 & -0.42 & +0.54 & -0.49 & +0.49 & -0.45 \\
\hline & $c$-jets TRF & +0.06 & -0.06 & +0.08 & -0.08 & +0.07 & -0.07 \\
\hline & light-jets TRF & +0.04 & -0.04 & +0.06 & -0.06 & +0.05 & -0.05 \\
\hline \multirow{6}{*}{ Others } & Z HF Scale Factor & +0.07 & -0.07 & +0.09 & -0.09 & +0.08 & -0.08 \\
\hline & Signal Modeling & +0.00 & -0.23 & +0.00 & -0.23 & +0.00 & -0.23 \\
\hline & $M C$ cross section & +0.04 & -0.04 & +0.04 & -0.04 & +0.04 & -0.04 \\
\hline & $M C$ statistics on signal & +0.01 & -0.01 & +0.01 & -0.01 & +0.01 & -0.01 \\
\hline & $M C$ statistics on background & +0.03 & -0.03 & +0.04 & -0.04 & +0.03 & -0.04 \\
\hline & $P D F$ & +0.02 & -0.02 & +0.02 & -0.02 & +0.02 & -0.02 \\
\hline \multicolumn{2}{|r|}{ Total } & +0.68 & -0.64 & +0.82 & -0.75 & +0.72 & -0.67 \\
\hline
\end{tabular}

Table 6.6: Summary of the total systematic uncertainties for the combined results (all channels). 


\section{Chapter 7}

\section{Conclusion}

The $t \bar{t}$ pair production cross section is measured by this analysis in the lepton+jets channel using a b-tagging algorithm for about $900 \mathrm{pb}^{-1}$ data taken at $\sqrt{s}=1.96 \mathrm{TeV}$. The combined result for all channels is

$$
\ell+\text { jets }: \quad \sigma_{p \bar{p} \rightarrow t \bar{t}+X}=8.05_{-0.53}^{+0.55} \text { (stat) }{ }_{-0.67}^{+0.72} \text { (syst) } \pm 0.49(\mathcal{L}) \mathrm{pb} .
$$

In this analysis, the $t \bar{t}$ pair production cross section is measured with $12.6 \%$ precison. The theoretical calculations $[7,8]$ have uncertainties of $6.2 \%$ and $11.9 \%$, respectively. Therefore, the experimental precision is close to the theoretical precision at present.

The experimental result will continue to increase in precision as additional data is acquired at the Tevatron. The LHC accelerator will also start soon and it is expected that one $t \bar{t}$ pair event per second will be created in the LHC. By making accurate measurements of the $t \bar{t}$ cross section, a precise comparison of the experimental result and the perturbative QCD calculation can be made, with any difference between theory and experiment being an indication of new physics beyond the Standard Model.

Moreover, $t \bar{t}$ pair production is the dominant background for Higgs boson production and the search for new physics beyond the Standard Model. Therefore, it is very important to understand 
accurately $t \bar{t}$ pair production in order to suppress the $t \bar{t}$ background and discriminate signal from background in these analyses.

Figure 7.1 shows a comparison of the $t \bar{t}$ production cross sections in other channels measured by the $\mathrm{D} \varnothing$ experiment and selected results from the CDF experiment. In the figure, the inner error bar indicates the statistical uncertainty and the outer error bar is the systematic uncertainty.

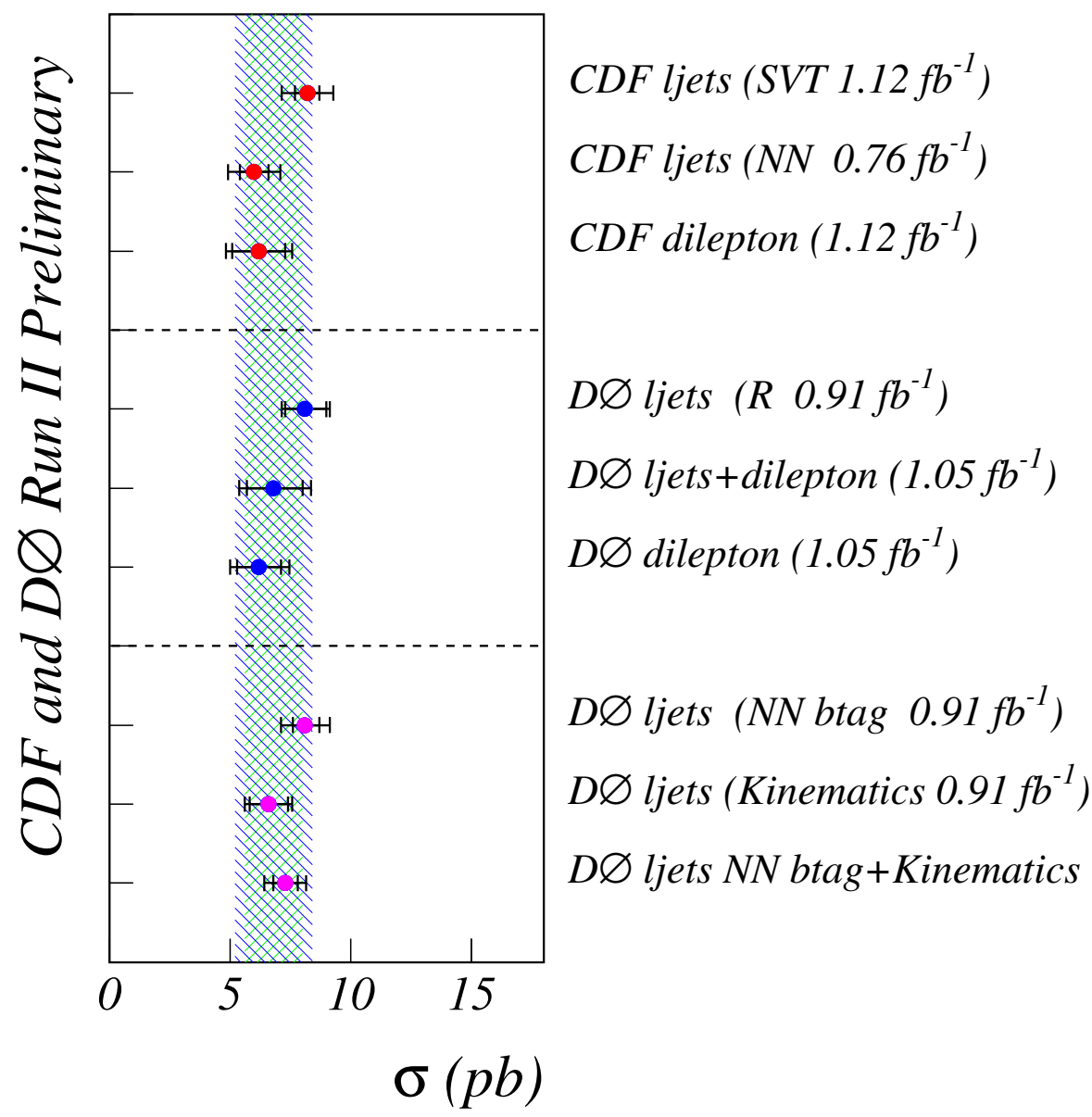

Figure 7.1: Summary of selected top quark pair production cross section measurements made by the $\mathrm{CDF}$ and $\mathrm{D} \varnothing$. The shaded area is the theoretical cross section with its uncertainty. 


\section{Appendix A}

\section{Calibration of the Luminosity}

\section{Monitor Readout Electronics}

The Luminosity Monitor (LM) detector, whose primary purpose is to determine luminosity at the $\mathrm{D} \emptyset$ detector, is briefly described in Section 2.2.5. The LM readout system consists of two parts: the front-end electronics mounted on the detector and the readout electronics located in the Moving Counting House $(\mathrm{MCH})$ at $\mathrm{D} \varnothing[69]$. In this appendix, the readout system and its calibration are described in detail.

\section{A.1 LM Readout Electronics}

\section{A.1.1 Configuration}

An overview of the LM readout system is shown in Figure A.1. As shown in the figure, the signal from the LM scintillators is readout by Hamamatsu photomultiplier tubes (PMT), which have a gain of approximately $10^{4}$ in the $1 \mathrm{~T}$ magnetic field at the LM detector. The signal pulse from the tubes is about $10 \mathrm{~ns}$ long. The preamplifiers amplify the signal by a factor of 60.5 . The amplified signal is sent to the MCH through high quality LMR-400 coaxial cables with lengths of about 200 ft. The signal is split in the $\mathrm{MCH}$, with $10 / 11$ of the signal sent to the VME-based electronics and 
1/11 of the signal sent to the NIM-based electronics [69]. Only the VME-based electronics will be described in this section.

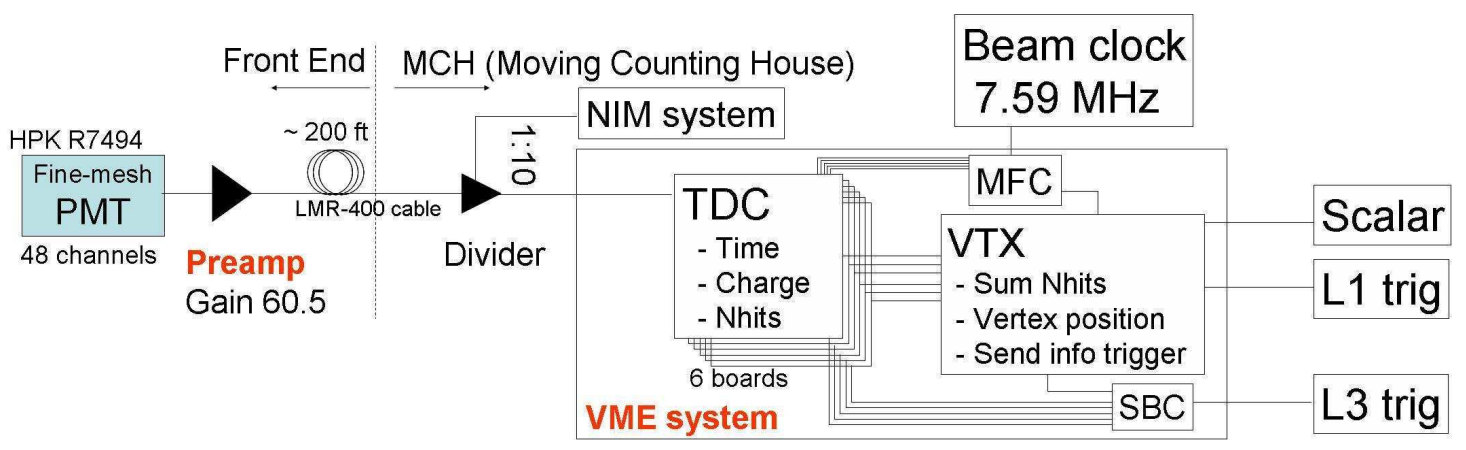

Figure A.1: Overview of the LM readout system [69].

There are two types of electronic boards in the VME-based readout system: the LM Timing (TDC) board and the LM Vertex (VTX) board. The role of the TDC board is to digitize the analog PMT signals and calculate several quantities from these signals described in Section A.1.2. The TDC board accepts signals from 8 PMTs and therefore 6 TDC boards are needed to readout the 48 LM counters (24 from the north array and 24 from the south array). The VTX board processes the outputs from the TDC boards and sends the processed data to the Level 1 Trigger Framework and the DAQ system. There is one VTX board in the LM readout system. Figure A.2 shows the LM VME crate and TDC and VTX boards. The VME crate is a 9U $280 \mathrm{~mm}$ deep crate [69] and has a standard DØ muon backplane [70]. The crate includes a PowerPC crate processor, a Single Board Computer (SBC) connected to the DAQ readout, a Muon Fanout Controller (MFC) that distributes timing signals and provides readout control, 6 TDC boards and one VTX board.

\section{A.1.2 TDC Board}

The TDC boards precisely measure the arrival time when a paricle hits the scintillator of the LM detector. The arrival time is converted into charge for each channel using a Time-to-Charge conversion technique. A current source $(10 \mathrm{~mA})$ is switched on when the input signal from the PMT passes through a progammable threshold and it is switched off when the common stop signal from 


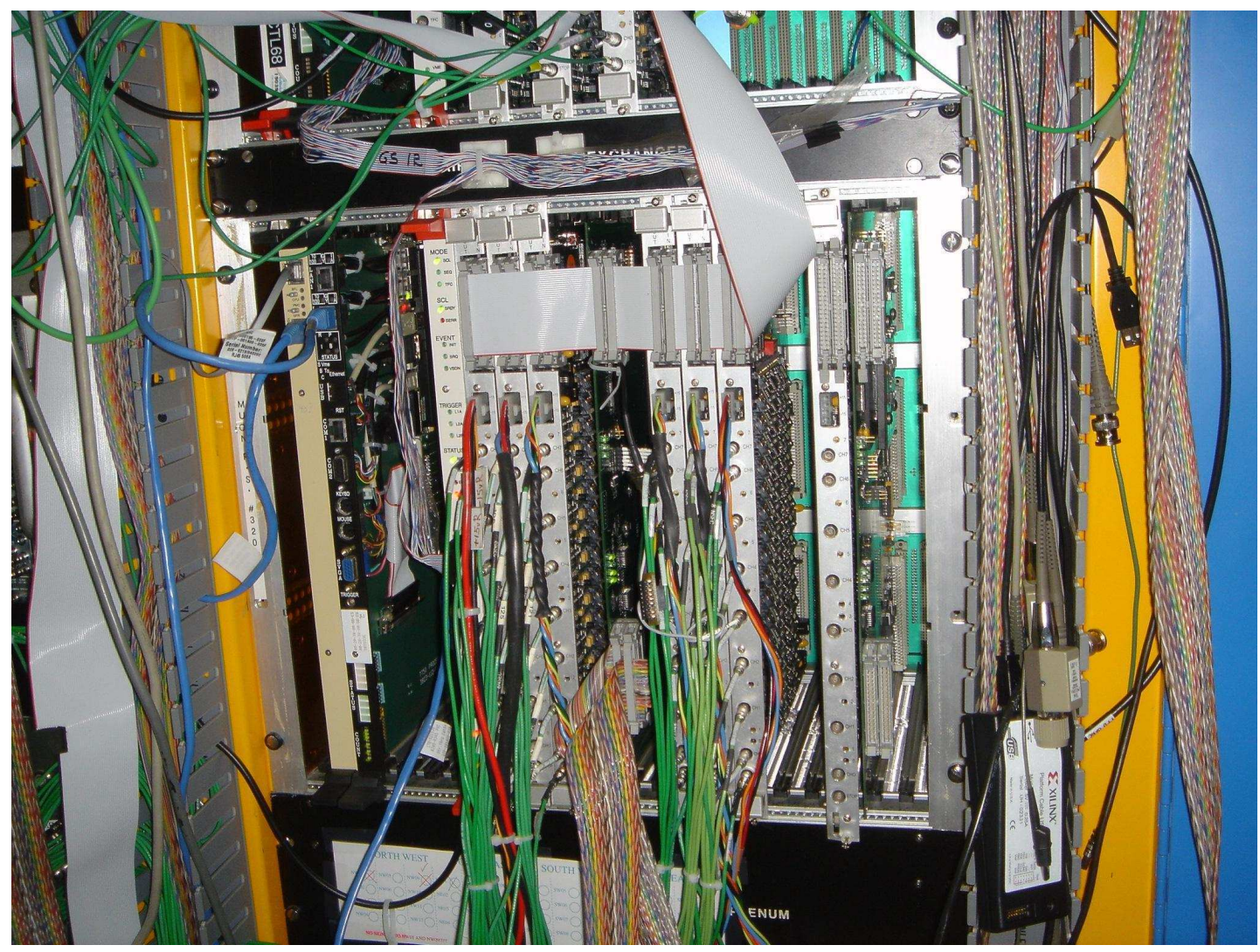

Figure A.2: Photograph of the LM VME crate. The PowerPC, SBC, MFC, three LM TDC boards for the north $(-\mathrm{z})$ detector, LM VTX board, and three LM TDC boards for the south $(+\mathrm{z})$ LM detector are shown from left to right [69]. 
the $\mathrm{D} \varnothing$ clock system is detected. The threshold voltage for each channel is determined by an 8-bit Digital-to-Analog Converter (DAC) that is VME addressable. The threshold is given by

$$
V_{T h}=0.1-\mathrm{DAC} / 640
$$

where $V_{T h}$ is the threshhold voltage $(\mathrm{V})$ and DAC denotes the DAC setting with 0 - 0xff range.

The current source is integrated and digitized using CAFÉ daughter cards, which were originally developed by the CDF collaboration for their calorimeter readout system. Two CAFÉ cards are allocated for each channel in the TDC board. One CAFÉ card is used to measure the PMT charge by integrating the current from the PMT. The other is used to measure the arrival time by integrating the current source between switch-on and switch-off. The former is called, the Charge CAFÉ and the latter is the Time CAFÉ. The CAFÉ card consists of the following main elements:

- An input buffer with about 50 ohm input impedence,

- A QIE chip to integrate the charge from the current source,

- An ADC to digitize the analog signal of the QIE, and

- A lookup table to produce a calibrated digital output.

The QIE chip, which was developed by Fermilab to provide multi-range pipelined charge integration, divides the input current into eight ranges: $1 / 2,1 / 4,1 / 8,1 / 16,1 / 32,1 / 64,1 / 128$ and 1/256, with the greatest to the lowest sensitivity from left to right. There is a four stage pipeline in the QIE, requiring four integration capacitors for each range. Table A.1 describes the QIE chip pipeline stages. As shown in the table, the charge is integrated on one of the four capacitors during the first

\begin{tabular}{|c|c|c|c|c|c|}
\hline \hline Capacitor & $\mathrm{t}=0 \mathrm{~ns}$ & $\mathrm{t}=132 \mathrm{~ns}$ & $\mathrm{t}=264 \mathrm{~ns}$ & $\mathrm{t}=396 \mathrm{~ns}$ & $\mathrm{t}=528 \mathrm{~ns}$ \\
\hline 0 & Integration & Range Select & Output & Reset & Integration \\
1 & Reset & Integration & Range Select & Output & Reset \\
2 & Output & Reset & Integration & Range Select & Output \\
3 & Range Select & Output & Reset & Integration & Range Select \\
\hline \hline
\end{tabular}

Table A.1: The operation on four capacitors in each stage. 
stage. The second stage examines the integrated charge for all eight ranges and then selects the optimal range. The integrated charge is driven onto the analog output during the third stage and the capacitors are reset during the last stage. The pipeline operates with a 132 ns clock.

The output of the QIE is sent to the Analog-Digital-Converter (ADC). A 10-bit flash ADC digitizes the integrated charge. The ADC output is used as the address of the lookup table memory. The lookup table memory is divided into two sections: "pass-through" and "calibrated". The passthrough table outputs the input address and is used for calibration of the CAFÉ card. The calibrated table provides the calibrated output. More details are discussed in Section A.2 on calibration.

The TDC board calculates an 8-bit corrected time signal by summing the measured time from the Time CAFÉ and a charge slewing correction from the Charge CAFÉ for valid hits. Using the corrected time values, the TDC calculates several quantities used as inputs by the VTX board, including the number of PMT hits with a valid time measurement (NHIT), the number of halo hits, and the sum of the slewing corrected times for valid hits. Seven Xilinx Spartan FPGAs (Xilinx XCS40PQ240-3) [69] are used to implement the functions of the TDC. Figure A.3 is a photograph of the TDC board. The 7 FPGAs are located around the edge of the board and the 16 CAFÉ cards are located in the middle.

\section{A.1.3 VTX Board}

The outputs of TDC boards are combined using a daisy chain approach. The three TDC boards receiving the signals from the north $(-\mathrm{z})$ LM detector and the other three TDC boards receiving the signals from the south $(+\mathrm{z})$ LM detector are separately chained together. The first TDC board in the chain is connected to a 40-conductor flat cable and the output is sent to the second TDC. The second and the third TDC boards receive the signal from the previous board and their outputs are the combined result. Therefore, the output of the third TDC is the final combined output for three TDC boards. It is sent to the VTX board as an input.

The purpose of the VTX board is to generate the LM coincidence signals that are used to calculate the luminosity. The VTX board also generates proton and antiproton halo signals which 


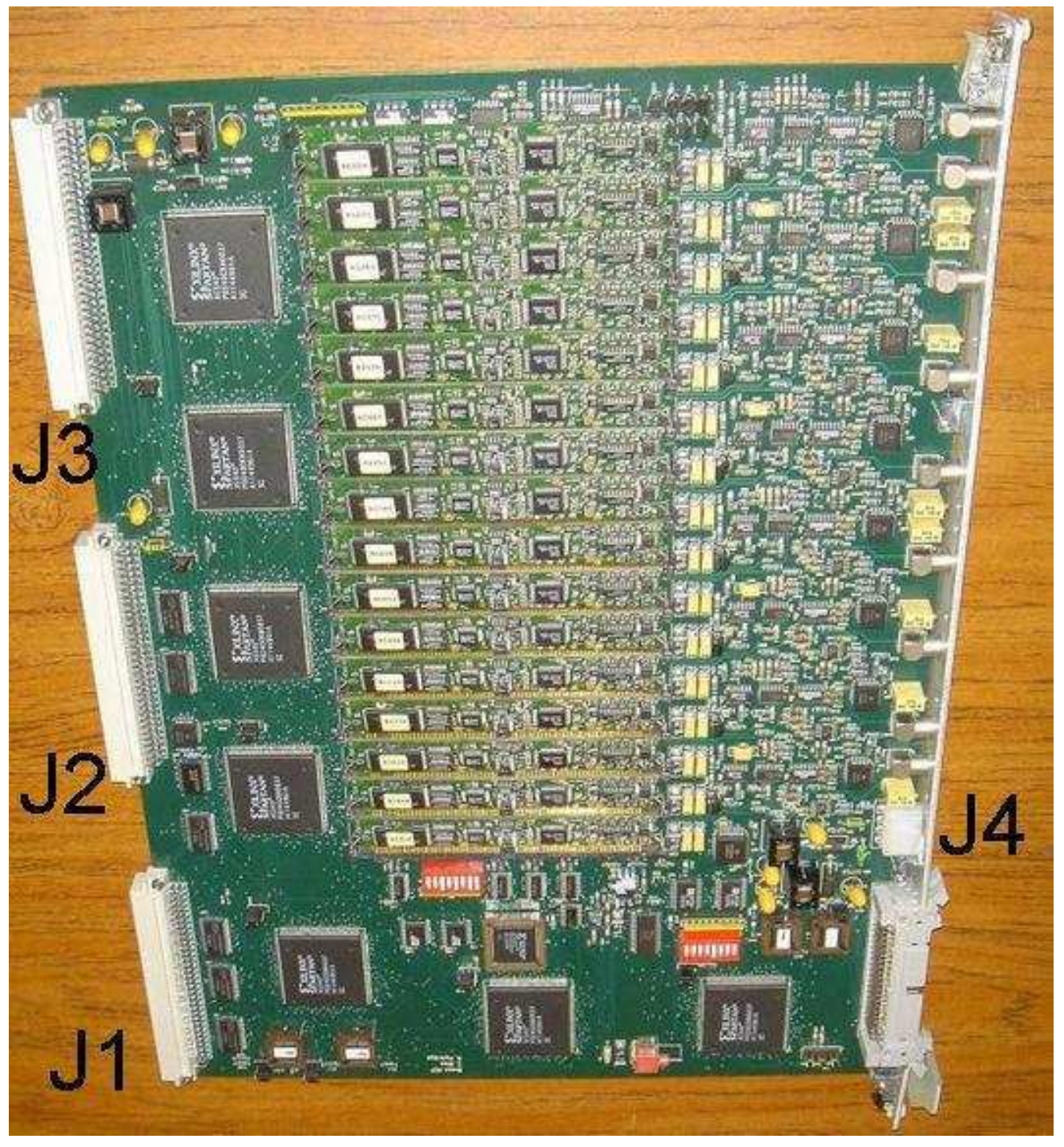

Figure A.3: Photograph of the TDC board [69]. 
are based on detecting early hits, and uses timing to estimate the position of the primary interaction vertex. Figure A.4 is a photograph of the VTX board.

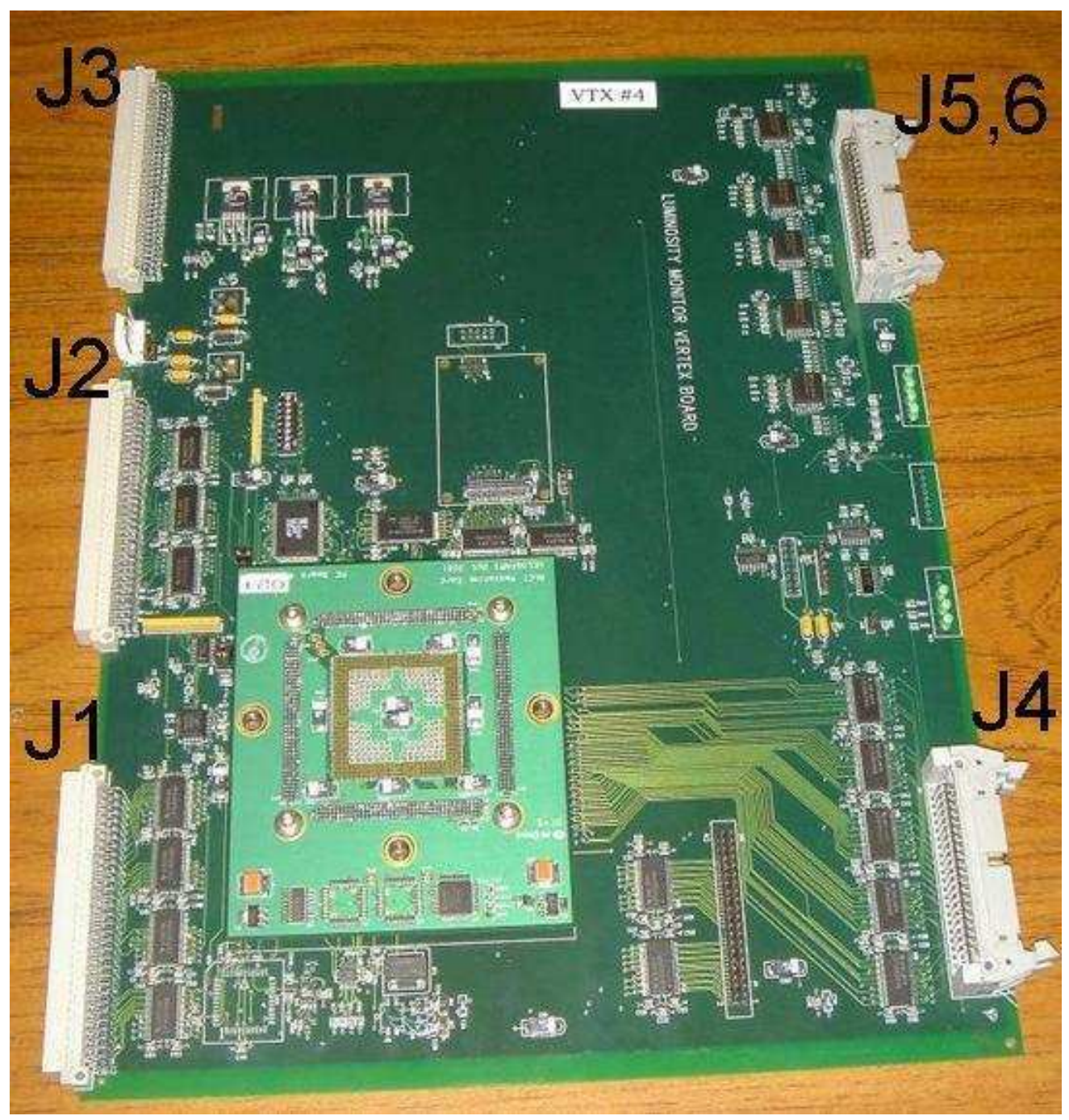

Figure A.4: Photograph of the VTX board [69]. The mounted circuit is the mezzanine card with the FPGA.

The functions of the VTX board are implemented using a single Xilinx Virtex FPGA, Xilinx XCV600E [69]. This FPGA implements the major logic functions and it is mounted on a mezzanine card which was developed by UCLA for the CMS muon trigger. A block diagram of the FPGA functionality is shown in Figure A.5. As shown in Figure A.5, the FPGA includes seven functional blocks as follows: 


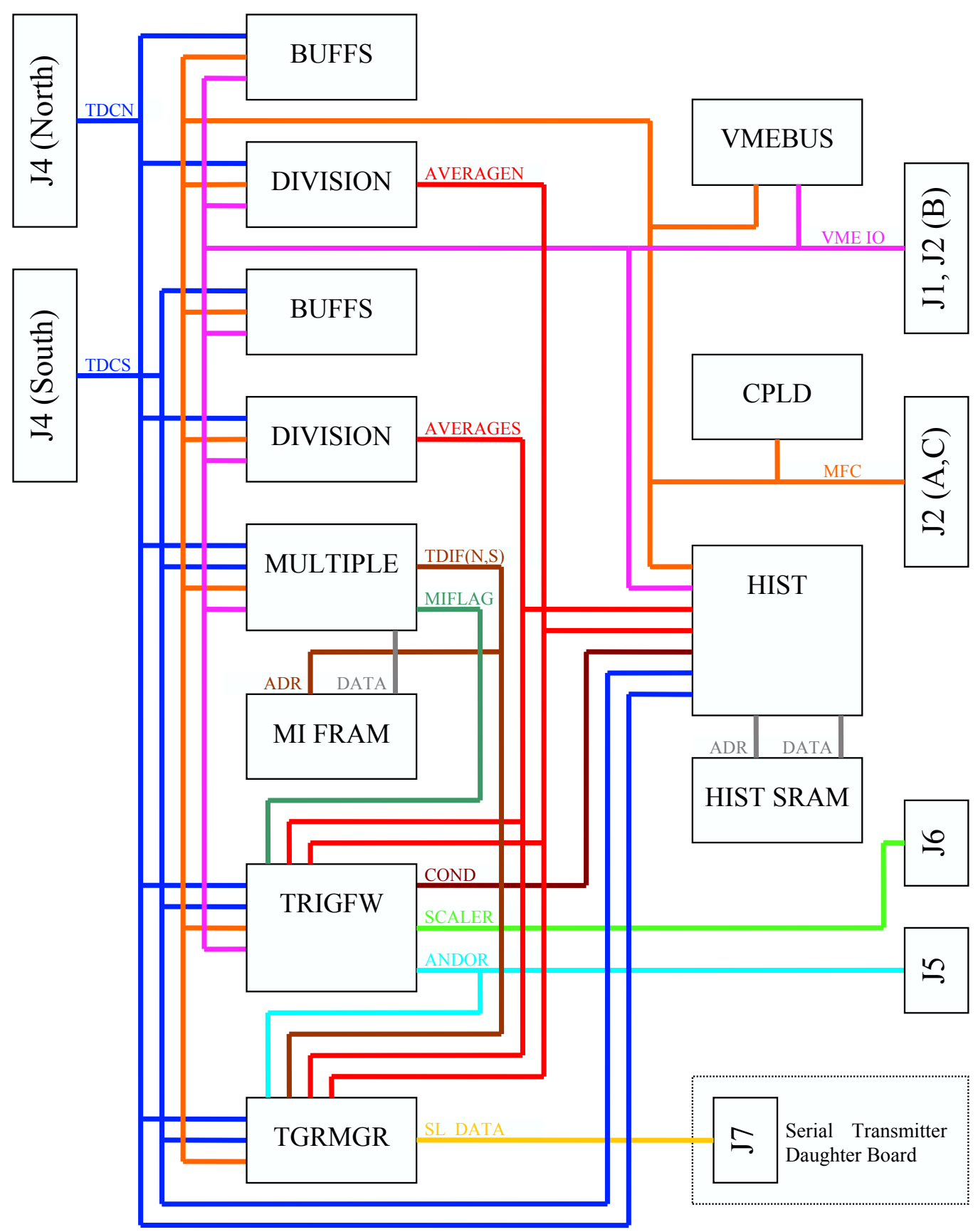

Figure A.5: High-level block diagram of the VTX board showing the flow of major signals. Signals are generally labeled near their source. In some cases, only a subset of the bus lines may be connected to a particular element [71]. 
- BUFFER: buffer the input signals from the TDC boards

- DIVISION: calculate the average time of the hits in the north and south LM arrays

- MULTIPLE: determine the multiple interaction (MI) flags which are used to identify whether there is a single or multiple interactions in a beam crossing

- TRIGFW: generate the signals set to the trigger framework, including the luminosity and halo scalar signals and the L1 trigger input terms from the LM

- TGRMGR: multiplex 96 bits of data into six 16-bits frame for use by the forward proton detector trigger system

- HIST: perform histogramming functions used for calibration, monitoring and diagnostics

- VMEBUS: provide the VME and MFC interface [71].

The HIST block is important for the calibration of readout electronics. It is discussed in the next section in detail.

\section{A.2 Calibration}

\section{A.2.1 CAFÉ Card Calibration}

The calibration of the LM readout electronics requires calibrating the CAFÉ cards on the TDC boards. The CAFÉ card has a variable current source for calibration. Using the current source, a known charge can be injected into the CAFÉ input and compared to the measured value after processing by the CAFÉ card. The true value of the injected calibration charge can be calculated using the following equation [72]

$$
Q=(132 n s) \times\left(\frac{V C A L}{2^{16}} \times 10 \mathrm{~V}\right) \times(1 \mathrm{~mA} / \mathrm{V})=(0.0201 \mathrm{pC}) \cdot V C A L
$$

where $V C A L$ denotes the value loaded into the 16-bit $V C A L D A C$ on the TDC board. The output of the $V C A L D A C$ is a voltage ranging between 0 and $10 \mathrm{~V}$. The calibration current source provides 
$1 \mathrm{~mA} / \mathrm{V}$ and is integrated for $132 \mathrm{~ns}$. Therefore, the integrated charge from the calibration current source is given by the Equation A.2.

On the other hand, the integrated charge is measured using the formula

$$
Q=Q_{0}+Q_{\text {slope }} \times A D C
$$

where $Q_{0}$ and $Q_{\text {slope }}$ are calibration constants which have to be determined. By comparing this integrated charge to the true value from Equation A.2, the CAFÉ card can be calibrated. The CAFÉ card is very close to linear and hence a linear fit is sufficient to calibrate the CAFÉ card. One CAFÉ card has 8 different ranges, each with 4 pipeline capacitors. Therefore, 32 sets of calibration constants are required for each CAFÉ card. Figure A.6 shows a plot of ADC vs. VCAL. The eight ranges are clearly visible, with each range exhibiting good linearity.

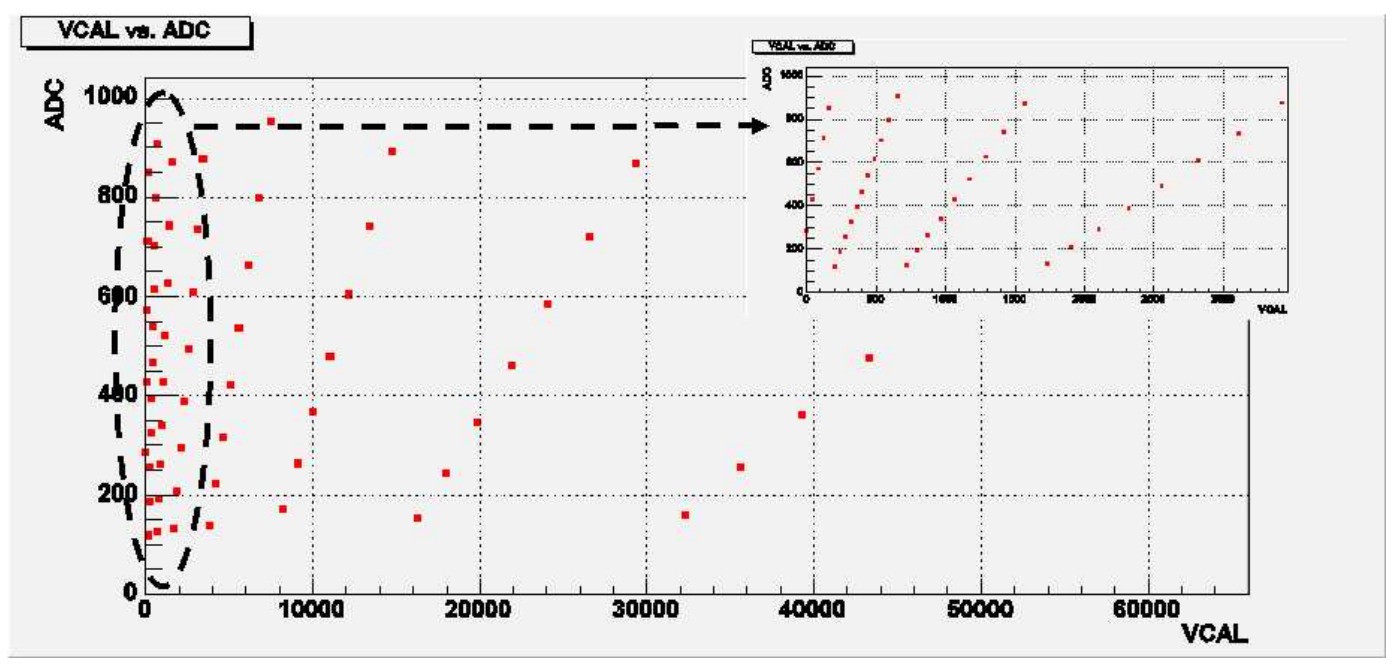

Figure A.6: ADC vs. VCAL taken from CAFÉ card for calibration.

The calibration data shown in Figure A.6 are taken using the "pass-through" mode of the lookup table. Table A.2 shows the format of the lookup table address. If the H/L bit is set to 1 ("passthrough" mode), the output of the lookup table is identical to the input address and the output (raw data) can be used for calibration. On the other hand, if the H/L is set to 0 ("calibrated" mode), the output of the lookup table provides the calibrated measurement. The CapID bits specify which of 


\begin{tabular}{|c|c|c|c|c|c|c|c|c|c|c|c|c|c|c|c|}
\hline 15 & 14 & 13 & 12 & 11 & 10 & 9 & 8 & 7 & 6 & 5 & 4 & 3 & 2 & 1 & 0 \\
\hline $\mathrm{H} / \mathrm{L}$ & CapID & \multicolumn{10}{|c|}{ Range } & \multicolumn{10}{|c|}{ ADC } \\
\hline
\end{tabular}

Table A.2: The lookup table address, 16 bits. 10 bits of 16 bits are ADC output. Range is the information from the QIE and the CapID is the capacitor ID of the QIE. H/L decides a mode of "pass-through" or "calibrated".

the four pipeline capacitors is used, and the range bits specify which of the eight ranges is selected. Figure A.7 shows a cartoon for the lookup table, and Figure A.8 shows the result of calibration (calibrated charge for all ranges in a CAFÉ card).

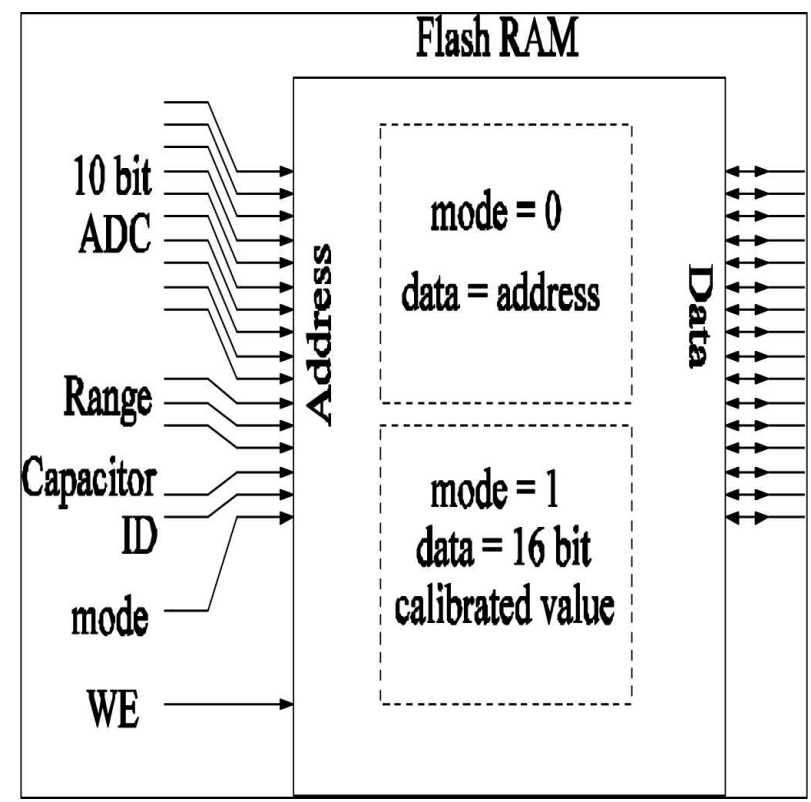

Figure A.7: Cartoon of the lookup table. Data flows from left to right. Mode $=1$ is "calibrated" and mode $=0$ is "pass-through" [73].

For calibration, the 16-bit output of the lookup table is sent to the histogrammer on the VTX board via the histogram bus. The histogram memory is $64 \mathrm{k} \times 32$-bits, allowing the output of the lookup table to be accumulated. The HIST block of the VTX board FPGA controls to access this memory. The histogram is accumulated by the following procedure: identifying the bin to be updated, reading the current value in the bin, adding one, and writing the updated value back into the histogram memory. 


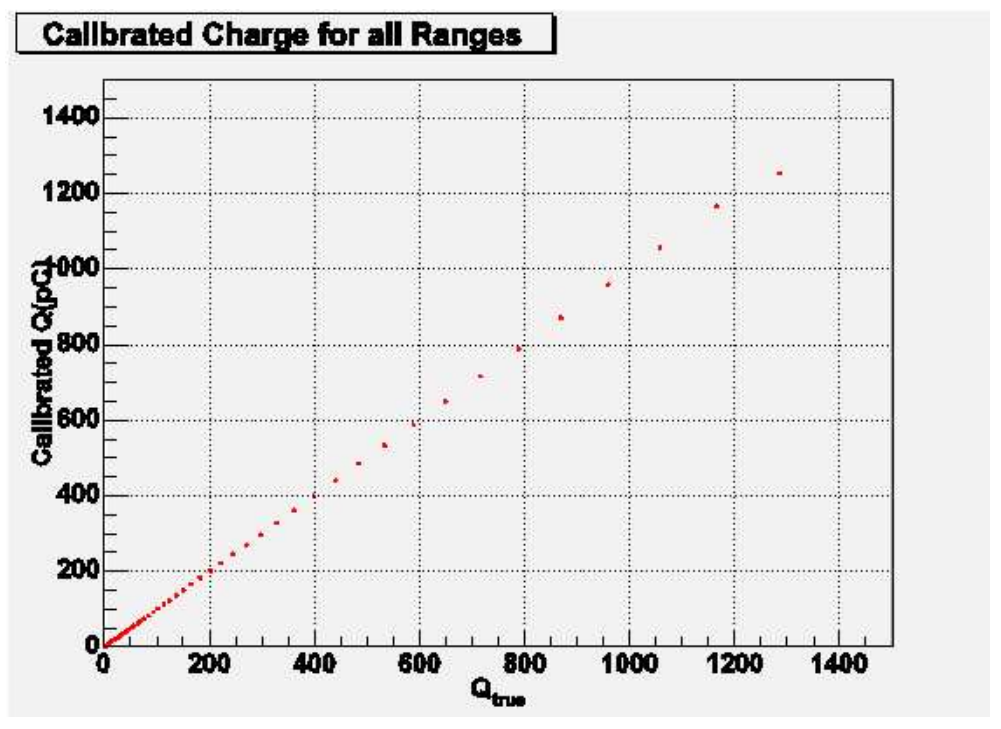

Figure A.8: A comparsion between the true integrated charge (xaxis) and the calibrated charge (y-axis) for all of the ranges in a CAFÉ card. It is observed to be almost linear.

There are a toal of 48 channels for the LM detector and each channel has 2 CAFÉ cards. Each CAFÉ card contains four pipeline capacitors, and 63 VCAL DAC values are measured in a typical calibration to cover the eight ranges. Around 18k entries per pipeline capacitor are accumulated for each VCAL DAC setting. Therefore, total number of charge measurements is $18 \mathrm{k} \times 63 \times 4 \times 2 \times 48=$ 435.5M. This huge number of entries can be taken during only one hour due to the histogramming feature in the VTX board.

The author was responsible for the CAFÉ calibration procedure. To perform the calibration, it is necessary to first set up the TDC and VTX boards to acquire data for a specific CAFÉ card and VCAL setting. To control and acquire calibration data efficiently, the author developed a Graphic User Interface (GUI) based histogramming controller, using python programming language. Figure A.9 shows a screen shot of the histogramming controller.

The biggest panel in the middle is the main controller and the others are pop-up panels from the main controller to set options such as the TDC board, channel, CAFÉ card (Time or Charge), and so on. The user can take calibration data channel by channel, and also can take data from all channels automatically by the click of one button. This GUI based controller communicates with 


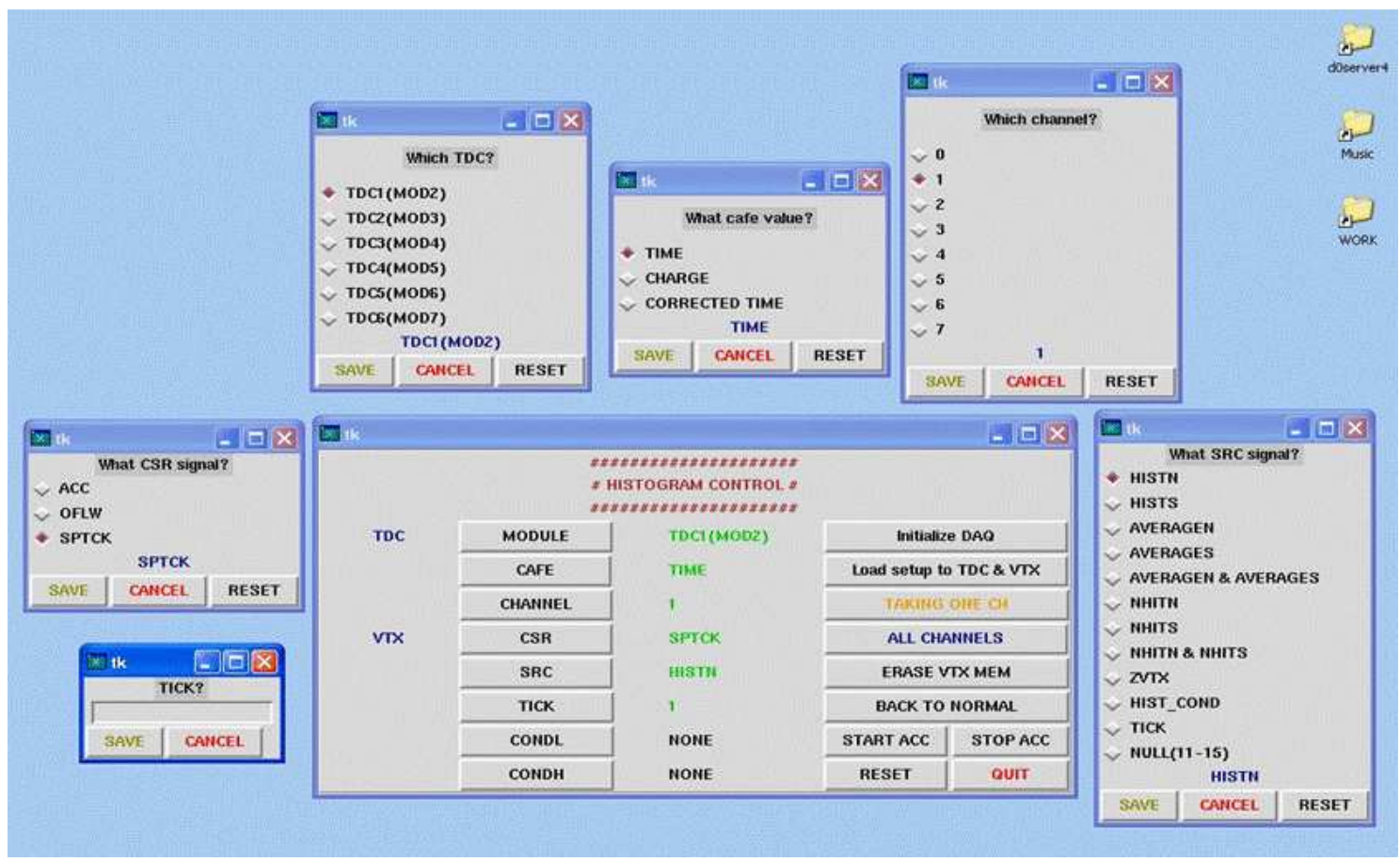

Figure A.9: The GUI based histogramming controller.

the VxWorks OS in the crate processor using EPICS control system.

Finally, the calibration constants are determined by linear fits of the calibration data and are stored with the format shown in Table A.3.

\begin{tabular}{|c|}
\hline TDC Module ID (1-6) \\
\hline Channel number for the TDC (0-7) \\
\hline QFlag (0 = Time, $1=$ Charge) \\
\hline Range (0-7) \\
\hline Capacitor ID (0-3) \\
\hline$Q_{0}(\mathrm{pC})$ \\
\hline$Q_{\text {slope }}(\mathrm{pC}$ /ADC count) \\
\hline RMS of Fit Residuals (pC) \\
\hline
\end{tabular}

Table A.3: The data format used to store the CAFÉ card calibration results [72].

\section{A.2.2 Global Time Calibration}

There is another important step for the LM readout system calibration, called "Global Time Calibration". This calibration provides the time-of-flight calibration for each channel using collider 
data.

To measure the precise arrival time of particles in the LM detector, the slewing correction is applied for the measured arrival time, and the corrected time is given by:

$$
T=T_{0}+T_{\text {slope }} \times Q_{T}-\frac{K}{\sqrt{Q_{P}}}
$$

where $Q_{T}$ is the integrated charge measured by Time CAFÉ, $Q_{P}$ is the integrated charge measured by Charge CAFÉ, and $T_{0}, T_{\text {slope }}, K$ are calibration constants determined by global fitting. The calibration constants for each channel can be calculated by minimizing the following $\chi^{2}$

$$
\chi^{2}=\sum_{\text {Events }}\left(\left\langle T^{N}\right\rangle-\left\langle T^{S}\right\rangle-\frac{2 z_{v}}{c}\right)^{2}+\frac{\lambda}{N_{\text {Hits }}} \sum_{\text {Events }} \sum_{\text {Counters }} T\left(T_{0}, T_{\text {slope }}, K\right)
$$

where $\left\langle T^{N}\right\rangle$ and $\left\langle T^{S}\right\rangle$ are the average arrival time for the north and south LM counters in the event respectively, $z_{v}$ is the $\mathrm{z}$ coordinate of the primary interaction vertex, and $\lambda$ is a Lagrange multiplier that is used to impose the constraint $\langle T\rangle=0$ and remove the ambiguity in the definition of $T=0$.

Equation A.5 is minimized for all 48 channels simultaneously by solving a system of $3 \times 48+1=$ 145 linear equations with 145 unknowns ( 1 is for $\lambda$ ). The results are stored for each channel with the format shown in Table A.4.

\begin{tabular}{|c|}
\hline TDC Module ID (1-6) \\
\hline Channel number for the TDC (0-7) \\
\hline$T_{0}(\mathrm{~ns})$ \\
\hline$T_{\text {slope }}(\mathrm{ns} / \mathrm{pC})$ \\
\hline$K$ ns pC $\left.^{1 / 2}\right)$ \\
\hline$Q_{0}(\mathrm{pC})$ \\
\hline RMS of Fit Residuals (pC) \\
\hline
\end{tabular}

Table A.4: The data format used to store the global time calibration results [72].

The calibrated outputs of the CAFÉ cards incorporate the results of the CAFÉ and global 
calibrations. For the Time CAFÉ, the measured time is given by:

$$
\text { Time }=T_{\text {off }}-N I N T\left(\frac{T_{0}+T_{\text {slope }} \cdot Q_{T}}{T_{l s b}}\right)
$$

where $T_{o f f}$ is the time lookup table offset, $T_{l s b}$ is the time binning, and NINT is the nearest integer function. The calibrated time is loaded into the lookup table with the format shown in Table A.5.

\begin{tabular}{|c|c|c|c|c|c|c|c|c|c|c|c|c|c|c|c|}
\hline 15 & 14 & 13 & 12 & 11 & 10 & 9 & 8 & 7 & 6 & 5 & 4 & 3 & 2 & 1 & 0 \\
\hline 0 & 0 & 0 & VT & \multicolumn{10}{|c|}{ Time } \\
\hline
\end{tabular}

Table A.5: Format of the Time CAFÉ lookup table output.

For the Charge CAFÉ, the charge slewing correction is given by:

$$
\text { Slew }=S_{o f f}-N I N T\left(\frac{K}{\sqrt{Q_{P}} \cdot T_{l s b}}\right)
$$

where $S_{\text {off }}$ is the charge lookup table offset. It is loaded into the lookup table with the format shown in Table A.6 [72]:

\begin{tabular}{|c|c|c|c|c|c|c|c|c|c|c|c|c|c|c|c|}
\hline 15 & 14 & 13 & 12 & 11 & 10 & 9 & 8 & 7 & 6 & 5 & 4 & 3 & 2 & 1 & 0 \\
\hline Q Range & \multicolumn{10}{|c|}{ Q Data } & \multicolumn{10}{|c|}{ Slewing Correction } \\
\hline
\end{tabular}

Table A.6: Format of the Charge CAFÉ lookup table output. 


\section{Appendix B}

\section{Soft Lepton Tagger with Neural}

\section{Network Method (SLTNN tagger)}

A new b-tagging algorithm, the "SLTNN" tagger is described in this appendix.

\section{B.1 Introduction}

The SLTNN tagger is a combination of the SLT tagger and a neural network (NN) method that includes both muon and lifetime variables. Around $20 \%$ of b-jets decay semi-leptonically with a muon in the final state $(b \rightarrow \mu X$ is $10.95 \%$ and $b \rightarrow c \rightarrow \mu X$ is $9.58 \%$ [3]). On the other hand, only a very small fraction of light-quark or gluon jets decay semi-leptonically, and therefore a jet can be identified as a b-jets if a muon is found inside the jet. This is called the Soft Lepton Tagging (SLT). The SLT tagger is a very powerful b-identification algorithm with a fake rate below $1 \%$.

The basic idea for the SLTNN tagger is to reduce the fake rate without significant b-tagging efficiency loss. The NN method is applied to do this. Using the NN method, the fake rate of this new tagger is decreased by about factor of 10 compared to the SLT tagger. 


\section{B.1.1 Samples}

The data samples shown in Table B.1 are used in this analysis.

\begin{tabular}{|l|c|c|}
\hline \hline Name & Skim Criteria & Number of Events \\
\hline MUinclusive & One loose $\mu p_{\mathrm{T}}>4 \mathrm{GeV}$ inside a 0.7 cone jet & $262 \mathrm{M}$ \\
QCD & Jet Triggers & $72 \mathrm{M}$ \\
\hline \hline
\end{tabular}

Table B.1: The data samples used in this analysis.

A summary of the MC samples used in this analysis is shown in Table B.2. The table includes the number of taggable jets in each MC sample, where the taggable jet is a calorimeter jet matched to a track jet within $\Delta R<0.5[74]$.

\begin{tabular}{|c|c|c|c|}
\hline \hline Name & $N_{\text {Events }}$ & $N_{\text {Jets }}$ & $N_{\text {Taggable Jets }}$ \\
\hline$t \bar{t}$ & 571,750 & $4,236,391$ & $3,162,736$ \\
$Z \rightarrow b \bar{b}$ & 105,250 & 187,034 & 136,183 \\
$Z \rightarrow b \bar{b}$ with $\mu$ & 105,750 & 210,418 & 135,445 \\
$Z \rightarrow c \bar{c}$ & 107,250 & 197,207 & 134,290 \\
$Z \rightarrow c \bar{c}$ with $\mu$ & 107,750 & 226,060 & 150,693 \\
$Z \rightarrow q \bar{q}$ & 103,750 & 192,170 & 138,843 \\
$Z \rightarrow q \bar{q}$ with $\mu$ & 107,000 & 279,548 & 175,247 \\
direct $b \bar{b} p_{\mathrm{T}}=20 \sim 40,40 \sim 80,80 \sim 160,160 \sim 320,320 \sim 980$ & 265,000 & 617,923 & 462,746 \\
direct $c \bar{c} p_{\mathrm{T}}=20 \sim 40,40 \sim 80,80 \sim 160,160 \sim 320,320 \sim 980$ & 239,287 & 561,735 & 413,329 \\
QCD $q \bar{q} p_{\mathrm{T}}=20 \sim 40,40 \sim 80,80 \sim 160,160 \sim 320,320 \sim 980$ & $2,067,711$ & $8,910,178$ & $7,176,256$ \\
$\gamma+$ jets $p_{\mathrm{T}}=20 \sim 980,40 \sim 980,80 \sim 980,160 \sim 980,320 \sim 980$ & $2,800,000$ & $4,022,662$ & $2,737,789$ \\
\hline \hline \multicolumn{2}{|l|}{}
\end{tabular}

Table B.2: MC samples which are used in this study

\section{B.1.2 Object Selection}

In order to ensure high quality of the reconstructed vertex, while keeping the efficiency high, the following PV selections are required:

- $\left|z_{P V}\right| \leq 60 \mathrm{~cm}$ (PV within the SMT fiducial region)

- At least three tracks fitted to the PV.

For jets, the following requirements are applied:

- taggable jet 
- Jet $p_{\mathrm{T}}>15 \mathrm{GeV}$

- Jet $|\eta|<2.5$

- JESMU correction

- Muon smearing for MC sample.

Four different muon definitions are used in this analysis (see Table B.3) and they are studied in parellel. Their kinematic requirements are as follows:

- Muon $p_{\mathrm{T}}>4 \mathrm{GeV}$

- Muon $|\eta|<2.0$

- $\Delta R$ between jet and muon $<0.5$.

\begin{tabular}{|c|c|c|c|}
\hline \hline Definition & Muon Quality & Muon Type & Central Track Matching \\
\hline loose & loose & nseg $>0$ & \\
medium & medium & $\mathrm{nseg}>0$ & $\chi^{2}<100$ \\
medium3 & medium & $\mathrm{nseg}=3$ & \\
tight & tight & $\mathrm{nseg}=3$ & \\
\hline \hline
\end{tabular}

Table B.3: Muon definitions used in this analysis

\section{B.1.3 Method Description}

The SLTNN tagger is the combination of the SLT tagger and the NN method. First, the SLT tagger (muon-tagging) is applied. Then, the NN method is applied to the muon-tagged jets. An outline of this study is as follows:

- Muon-tagging

- NN method: find operating points for each muon definition using the NN output

- Tagging Efficiency: measure the scale factor and tagging efficiency for the data sample using the system 8 method 
- Fake Rate: measure the fake rate using the negative tag rate (NTR) method

- Estimation of systematic uncertainties.

\section{B.2 Neural Network Method}

Starting with the muon-tagged jets, the NN method is then applied. There are 11 input variables for the NN training. Table B.4 shows the variables and their descriptions. In the table, SVT $T^{\text {mutrk }}$ means the only secondary vertex (SV) that is reconstructed using the tracks which the muon track is included in. The reason why this restriction is applied is to minimize the correlation between the SLTNN tagger and the lifetime tagger. The absence of correlation between them is very important for the system 8 method [37]. The system 8 method is needed to measure the b-tagging efficiency in the data sample, and it will be discussed in the next section.

\begin{tabular}{|c|c|}
\hline \hline Input Variable & Description \\
\hline Muon $p_{\mathrm{T}}$ & Muon $p_{\mathrm{T}}$ in a jet \\
Muon $p_{\mathrm{T}}^{r e l}$ & Muon $p_{\mathrm{T}}$ measured relative to the jet axis \\
Muon $\Delta R$ & $\Delta R$ between the muon and the nearest jet \\
Muon $\chi^{2}$ & Muon central matching $\chi^{2}$ \\
Muon IP sig & Impact parameter (IP) significance of the muon \\
$S V T_{S L}^{\text {mutrk }} D L S$ & Decay length significance of the SV \\
$S V T_{S L}^{\text {mutrk }}$ Mass & Mass of the SV \\
$S V T_{S L}^{\text {mutrk }} \chi_{\text {ndof }}^{2}$ & $\chi^{2}$ per degree of freedom of the SV \\
$S V T_{S L}^{\text {mutrk }} N_{\text {tracks }}$ & Number of tracks used to reconstruct the SV \\
$S V T_{S L}^{\text {Sutrk }} N_{\text {vertices }}$ & Number of SV found in the jet \\
$C S I P$ Pomb & Weighted combination of the tracks' IP significances \\
\hline \hline
\end{tabular}

Table B.4: NN input variables and descriptions. The SL denotes the SuperLoose operating point for the SVT tagger.

The distributions for the NN input variables for signal (direct bb) and background (QCD and $\gamma+$ jets combined) in MC samples are shown in Figures B.1 and B.2. The figures are for the medium muon definition. A comparison of variables between the direct bb MC sample and the MUinclusive data sample of b-jets is shown in Figures B.3 and B.4. The following selections are applied to the MUinclusive sample in order to make an enriched $b \bar{b}$ sample in the figures: 
- away tagging with JLIPprob $<0.01$ (b-enriched sample)

- JLIPprob $<0.01$ on a taggable jet

where it is called as an away tag if a jet is taggable and it has only one other back-to-back taggable jet which passes some tagging criteria (JLIPprob $<0.01$ in this analysis) in the same event.
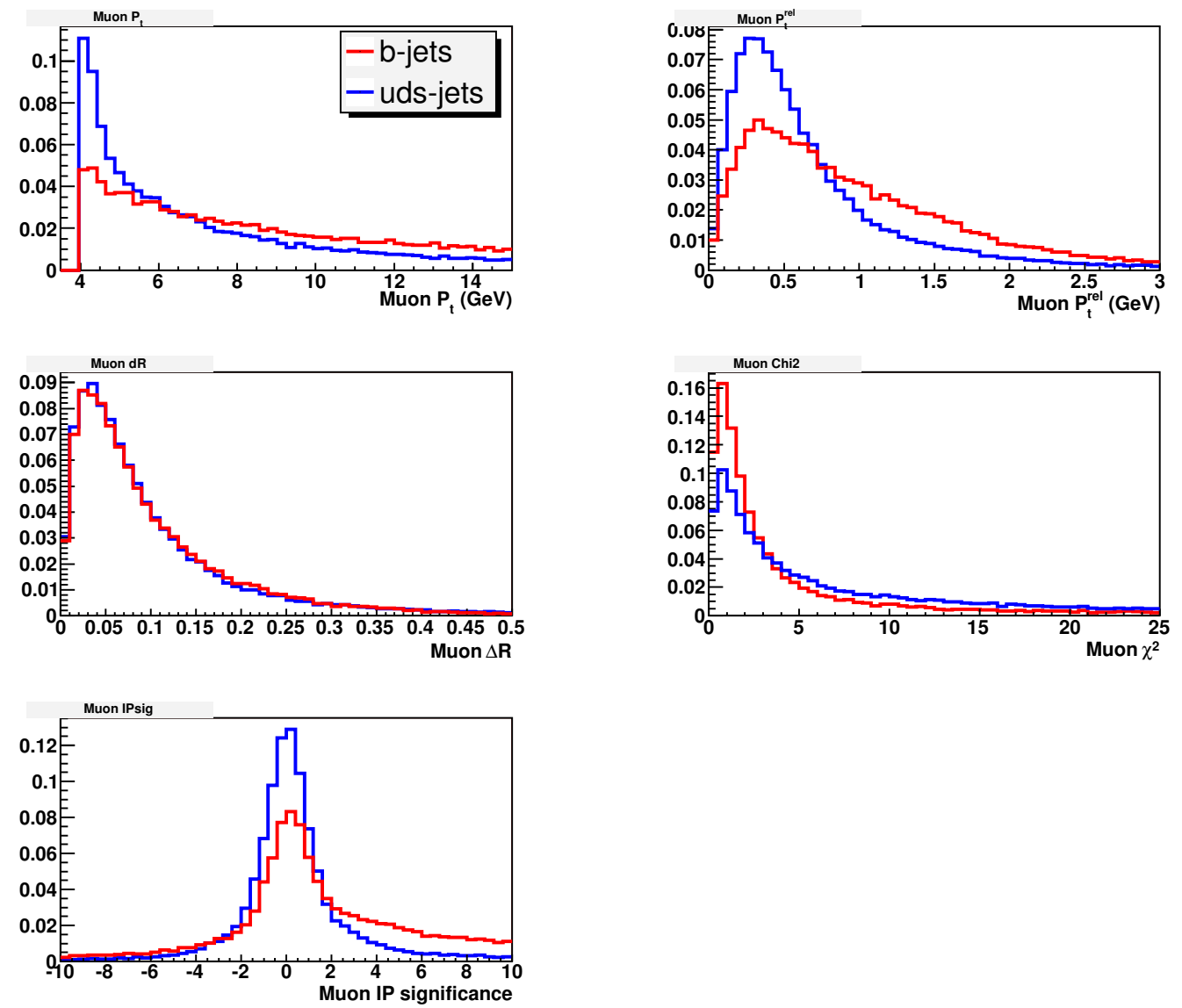

Figure B.1: The distribution of NN input variables for the Medium muon definition. Muon variables. From left to right, muon $p_{\mathrm{T}}, p_{t}^{r e l}, \Delta R$ (muon, jet), $\chi^{2}$ and IP significance. The red histogram is for b-jets and the blue histogram is for uds-jets.

Using the NN input variables, the NN training is performed with 500 epochs for each muon definition. The hidden nodes for the training is set to $24: 1$. The outputs for the NN training are shown in Figure B.5. 

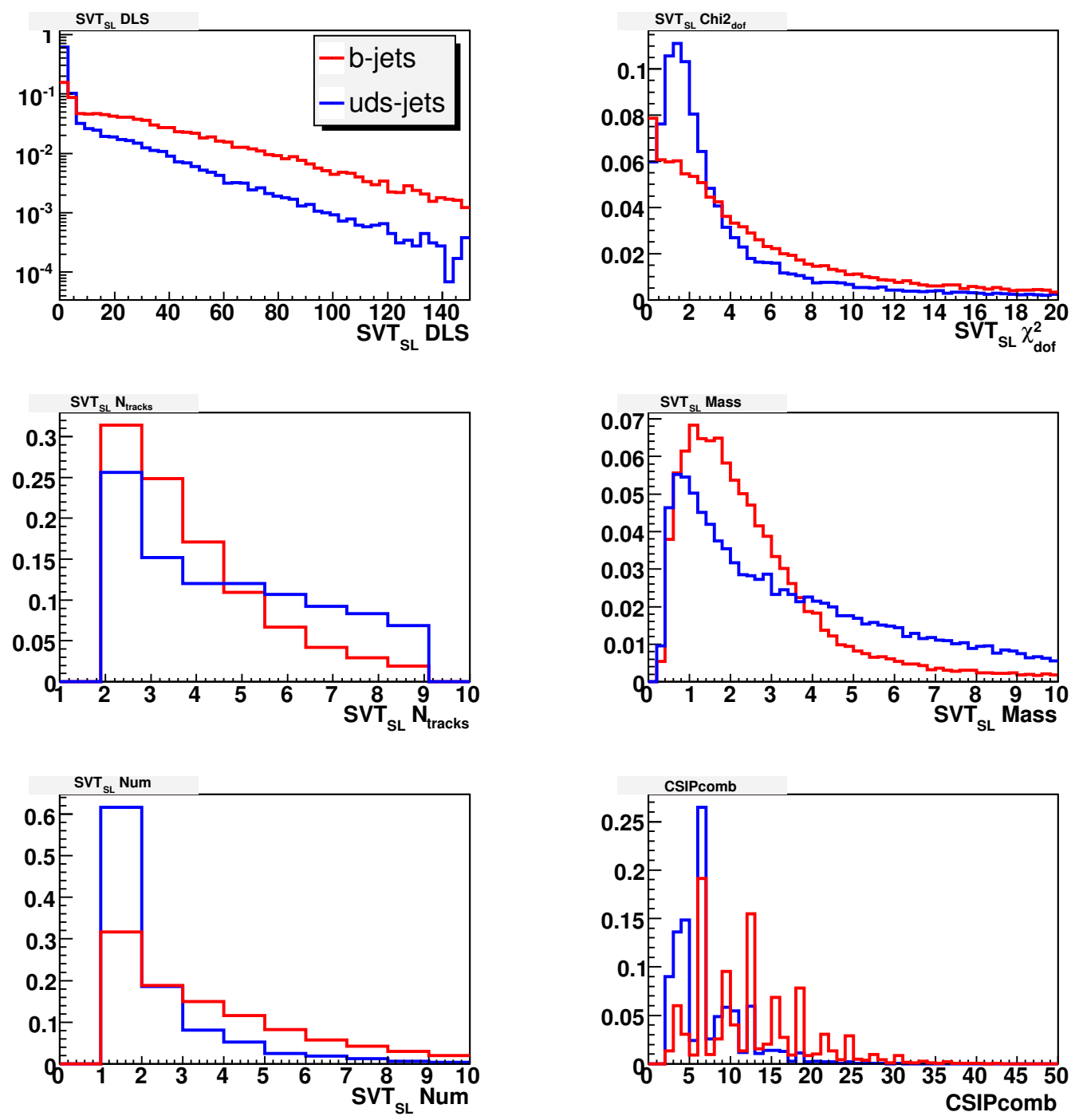

Figure B.2: The distribution of NN input variables for the Medium muon definition. SVT variables. From left to right, $S V T_{S L}^{m u t r k} D L S, S V T_{S L}^{m u t r k} \chi_{n d o f}^{2}, S V T_{S L}^{m u t r k} N_{\text {tracks }}, S V T_{S L}^{m u t r k} M a s s$, $S V T_{S L}^{m u t r k} N u m$, and CSIPcomb The red histogram is for b-jets and the blue histogram is for uds-jets. 

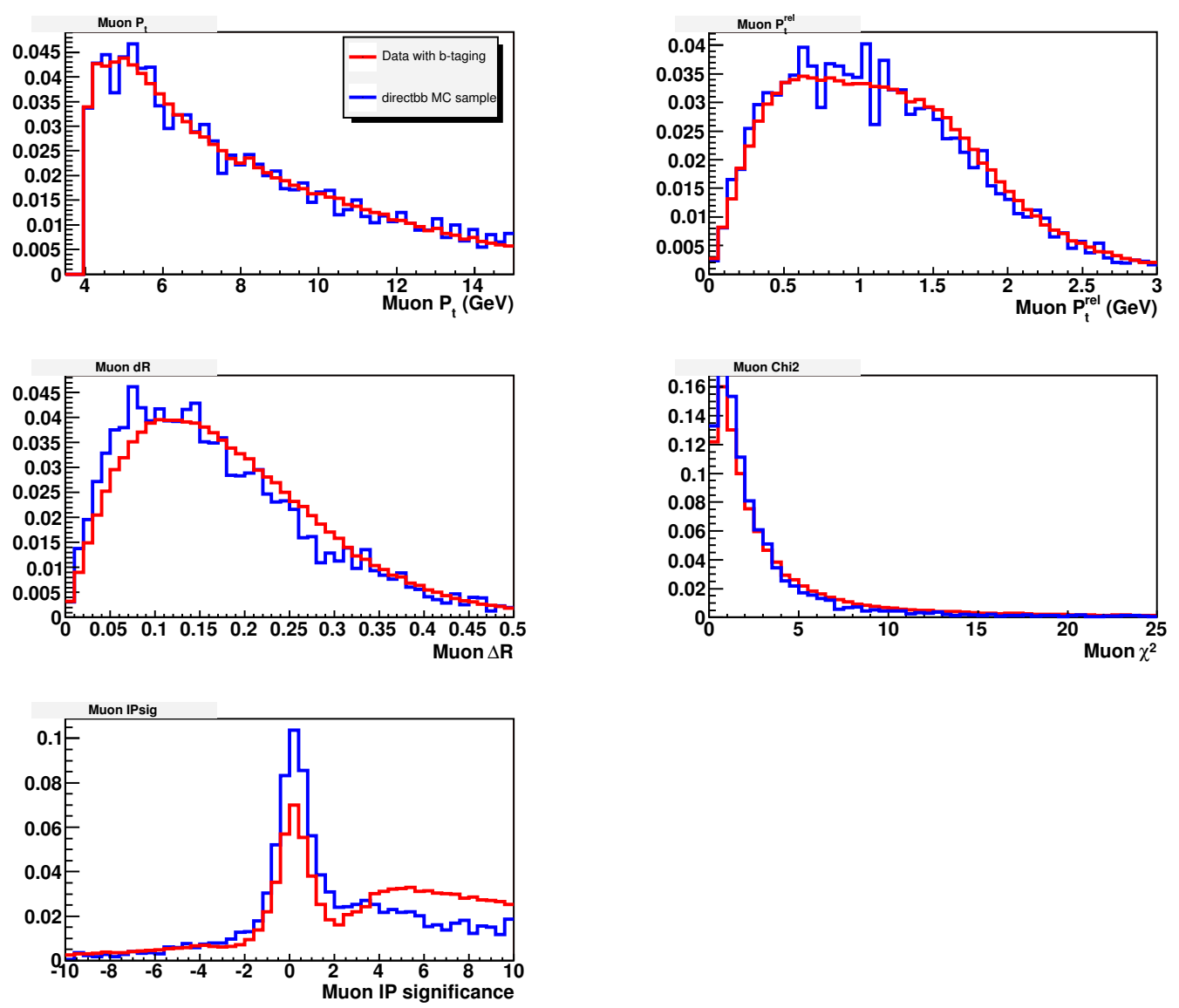

Figure B.3: Comparison of the distributions for the NN input variables between data and $\mathrm{MC}$ sample on b-jets for Tight muon definition. Muon variables. From left to right, muon $p_{\mathrm{T}}, p_{t}^{r e l}$, $\Delta R$ (muon, jet), $\chi^{2}$ and IP significance. The red histogram is for b-jets from data sample and the blue histogram is for b-jets from the directbb MC sample. 

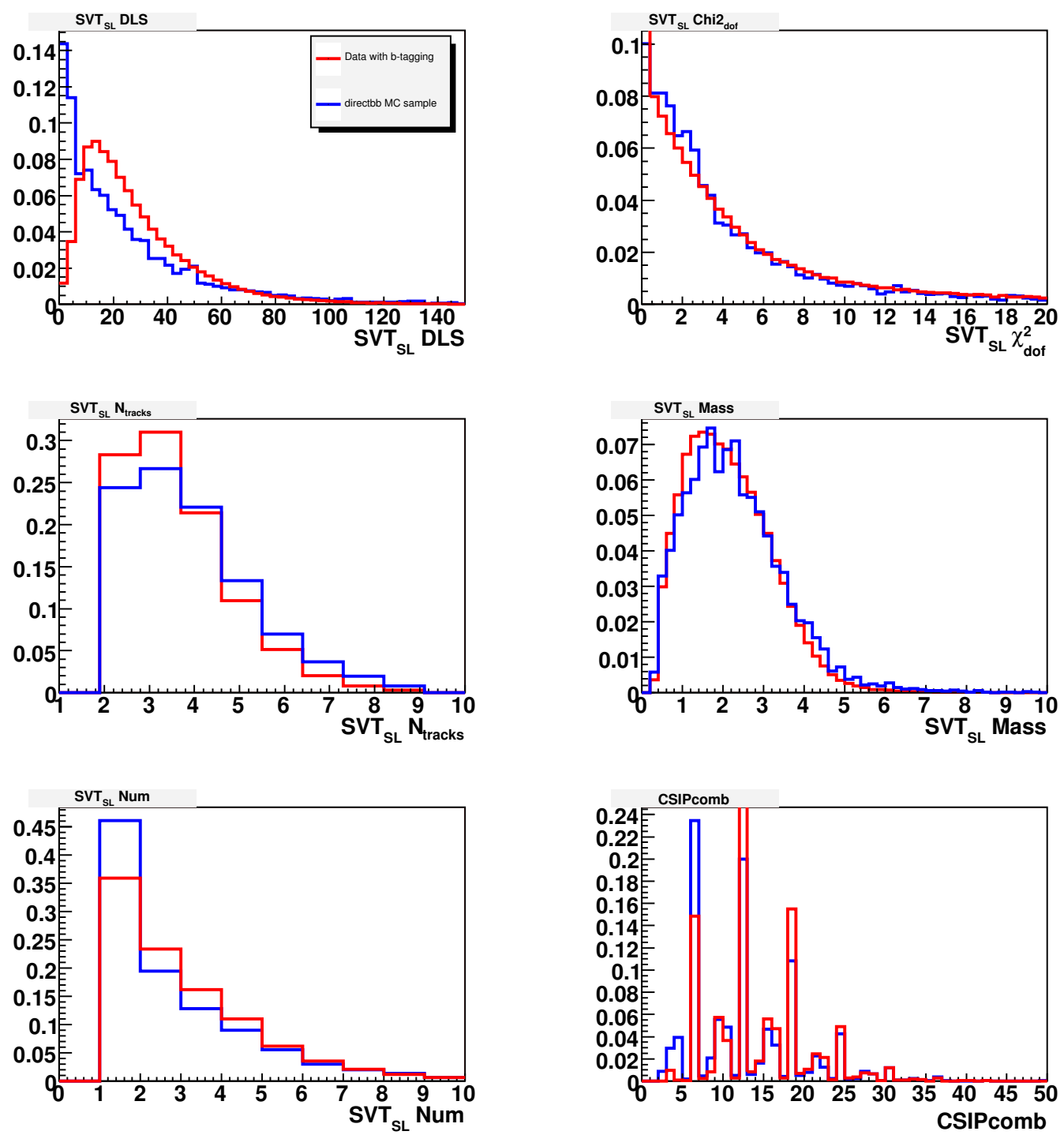

Figure B.4: Comparison of the distributions for the NN input variables between data and MC sample on b-jets for Tight muon definition. SVT variables. From left to right, $S V T_{S L}^{\text {mutrk }} D L S$, $S V T_{S L}^{\text {mutrk }} \chi_{\text {ndof }}^{2}, S V T_{S L}^{\text {mutrk }} N_{\text {tracks }}, S V T_{S L}^{\text {mutrk }}$ Mass, SVT $T_{S L}^{\text {mutrk }} N u m$, and CSIPcomb. The red histogram is for b-jets from data sample and the blue histogram is for b-jets from the directbb MC sample. 

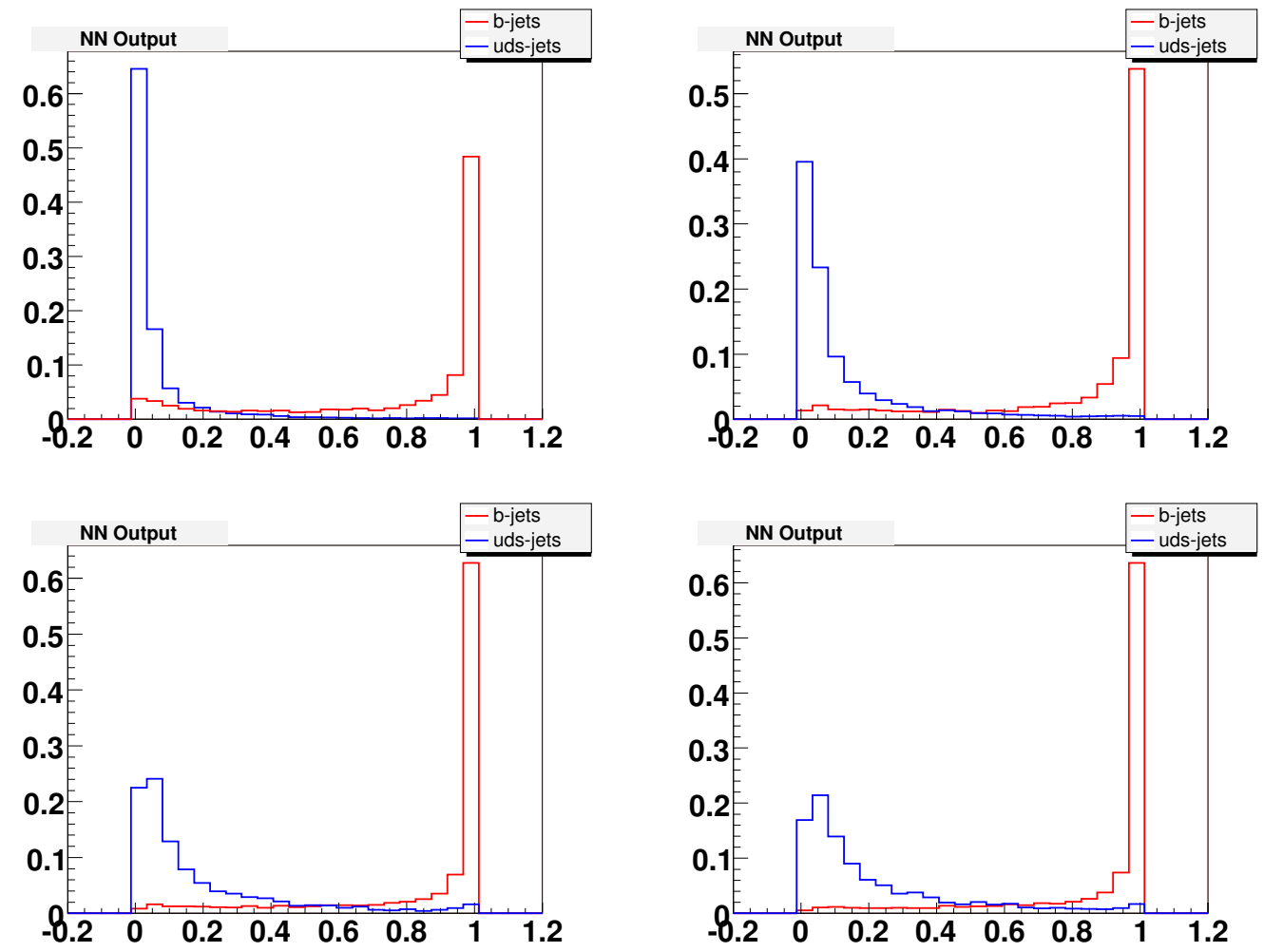

Figure B.5: Outputs for the NN training for each muon definition: Loose (top left), Medium (top right), Medium3 (bottom left) and Tight (bottom right). The red histogram is background (light-jets) from the QCD and $\gamma+$ jets combined MC samples, and the blue histogram is signal (b-jets) from the direct bb MC sample. 
The final b-tagging efficiency for the SLTNN tagger is

$$
\varepsilon_{S L T N N}=\varepsilon_{\text {muon-tagging }} \times \varepsilon_{N N}
$$

namely, the combination of both muon-tagging efficiency and pure NN tagging efficiency. Figure B.6 shows the final tagging efficiency of the SLTNN tagger $\left(\varepsilon_{S L T N N}\right)$ for b-jets. Figure B.7 shows the plot for the c-tagging efficiency. To measure the tagging efficiency and fake rate, the direct bb MC sample is used as signal and the QCD and $\gamma+$ jets combined MC sample is used as background. Three NN operating points (Loose, Medium, Tight) for each muon definition are selected by considering the fake rate, $0.15 \%$ for Loose, $0.10 \%$ for Medium and $0.04 \%$ for Tight. The NN cuts of the operating points are shown in Table B.5.

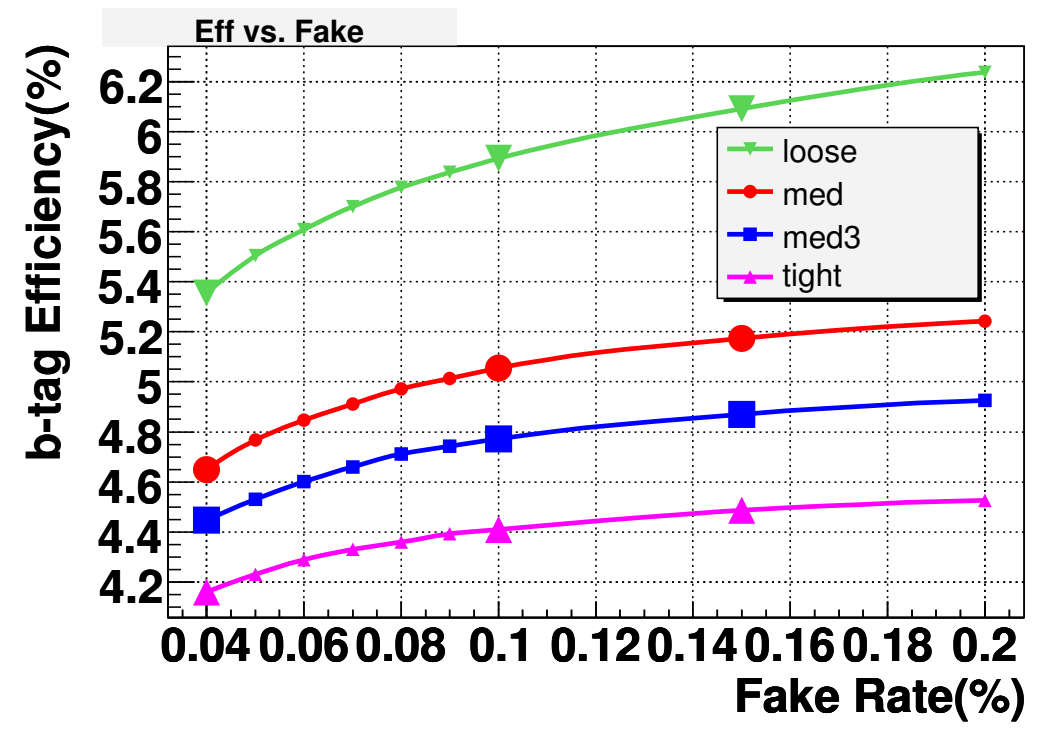

Figure B.6: 2D plot for Efficiency vs. Fake Rate measured in MC samples. The $\mathrm{y}$-axis is the b-tagging efficiency (the SLTNN tagging efficiency) measured in the direct bb MC sample and the $\mathrm{x}$-axis is the fake rate determined in the QCD $+\gamma+$ jets combined MC sample. The large points indicate the 3 operating points (Tight, Medium, and Loose from left to right) in this study for each muon definition. 


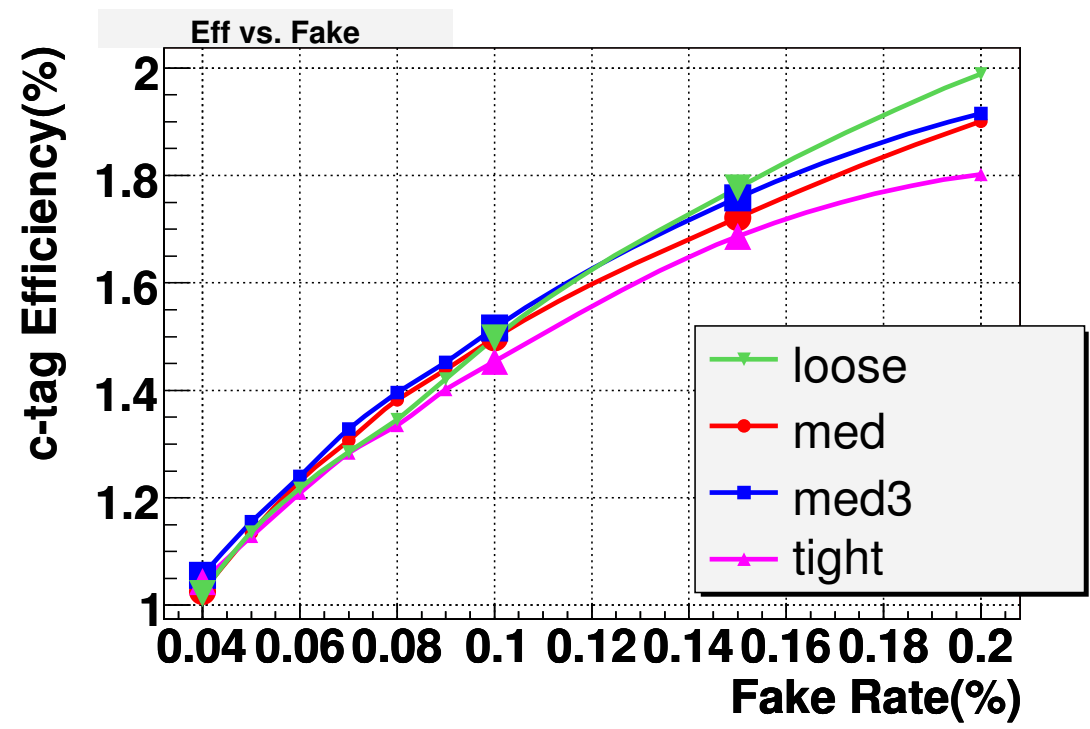

Figure B.7: 2D plot for Efficiency vs. Fake Rate measured in MC samples. The $\mathrm{y}$-axis is the c-tagging efficiency (the SLTNN tagging efficiency) measured in the direct cc MC sample and the $\mathrm{x}$-axis is the fake rate determined in the QCD $+\gamma+$ jets combined MC sample. The large points indicate the 3 operating points (Tight, Medium, and Loose from left to right) in this study for each muon definition.

\begin{tabular}{|c|c|c|c|c|}
\hline \hline operating points & Loose & Medium & Medium3 & Tight \\
\hline Loose & 0.172 & 0.089 & 0.067 & 0.045 \\
Medium & 0.258 & 0.160 & 0.134 & 0.114 \\
Tight & 0.505 & 0.429 & 0.394 & 0.375 \\
\hline \hline
\end{tabular}

Table B.5: The NN cuts for the operating points for each muon definition.

\section{B.3 Tagging Efficiency}

\section{B.3.1 Muon Tagging Efficiency in the MC Samples}

The muon-tagging efficiency in the MC samples can be simply estimated by dividing the number of b-jets (c-jets) passing the muon selection by the total number of b-jets (c-jets) in MC sample,

$$
\begin{aligned}
& \varepsilon_{\text {muon-tagging }}^{\text {bjets }}=\frac{N_{\text {passed muon selection }}^{\text {bjets }}}{N_{\text {total }}^{\text {bjets }}} \\
& \varepsilon_{\text {muon-tagging }}^{\text {cjets }}=\frac{N_{\text {passed muon selection }}^{\text {cjets }}}{N_{\text {total }}^{\text {cjets }}} .
\end{aligned}
$$


The muon-tagging efficiency has a different dependance on jet $p_{\mathrm{T}}$ for each $\eta$ region $(\mathrm{CC}, \mathrm{ICR}$, EC). Therefore, the jet $p_{\mathrm{T}}$ dependent muon-tagging efficiency is measured separately for each $\eta$ region. Figure B.8 shows the muon-tagging efficiency vs jet $p_{\mathrm{T}}$ for each $\mathrm{MC}$ sample $(t \bar{t}, Z \rightarrow b b(c c)$, direct $\mathrm{bb}(\mathrm{cc}))$. There are discrepancies between the MC samples with respect to the muon-tagging efficiency. This MC sample dependence is added as a systematic uncertainty.

To measure the muon-tagging efficiency, the muon correction factors, muon reconstruction and muon track-matching described in Section 3.1, are applied. The certified correction factors provided by the muon ID group are used in this analysis [24].

The systematic uncertainties in the muon-tagging efficiency originate from two sources: MC sample dependence and muon correction factors. The former is estimated by a closure test to compare the difference between direct tagging and applying $T R F_{\text {muon-tagging }}$ shown in Figure B.8. The latter is determined by varying the muon correction factors (muon id reconstruction and muon track matching) by $\pm 1 \sigma$ errors. Systematic uncertainties are individually measured for several jet $p_{\mathrm{T}}$ bins. Figure B.9 shows the systematic uncertainties for each $\eta$ region and $p_{\mathrm{T}}$ bins.

\section{B.3.2 System8 Method}

To measure the tagging efficiency in the data sample, the so-called "system8" method is used in this analysis [37]. The system 8 method consists of 8 equations with 8 unknowns, and the tagging efficiency of the SLTNN tagger in the data sample is one of the 8 unknowns. The data tagging efficiency can be measured with almost no input from MC simulations by using the system8 method.

The system8 method needs two independent taggers and two different data samples. In this study, the SLTNN tagger and JLIP (Jet Lifetime Probability) tagger are used. JLIP is the probability that the jet orignates from the PV. The SLTNN tagger uses the some of variables for the secondary vertex (lifetime information), therefore the SLTNN tagger is correlated with the JLIP. To reduce the correleation (it it assumed that two taggers are totally uncorrelated in the system8 method), only SV information not involving the muon track $\left(S V T^{m u t r k}\right)$ are used in the SLTNN tagger. The JLIP tagger is also modified by removing the muon track in the JLIP probability, and is called 

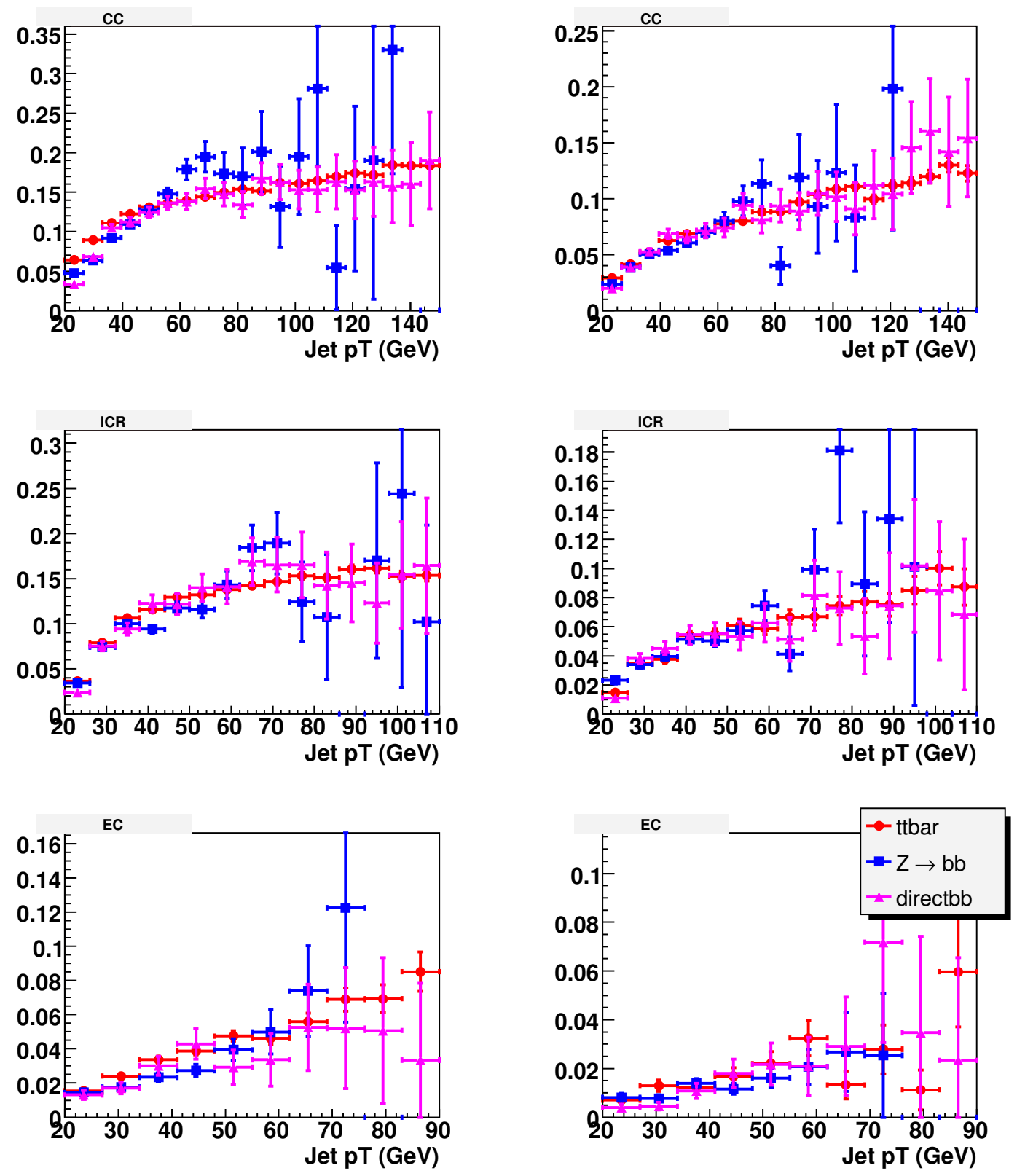

Figure B.8: The muon-tagging efficiency for b-jets (Left) and c-jets (right), Loose muon definition. The red histogram denotes $t \bar{t} \mathrm{MC}$ sample, the blue histogram denotes $Z \rightarrow b b(c c)$, the purple histogram denotes direct $\mathrm{bb}(\mathrm{cc})$ sample. From top to bottom, plots show the muon-tagging effciencies for CC, ICR, and EC regions, respectively. 

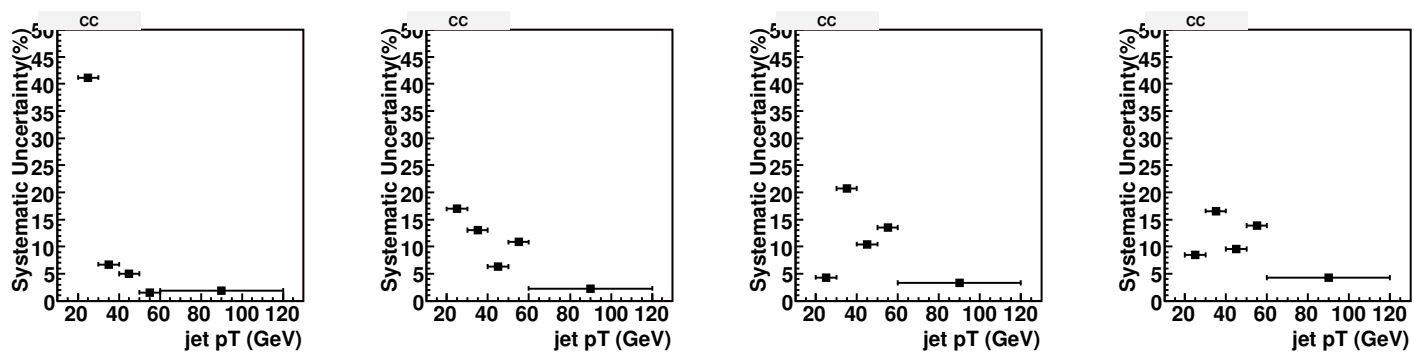

(a) CC $\eta$ region
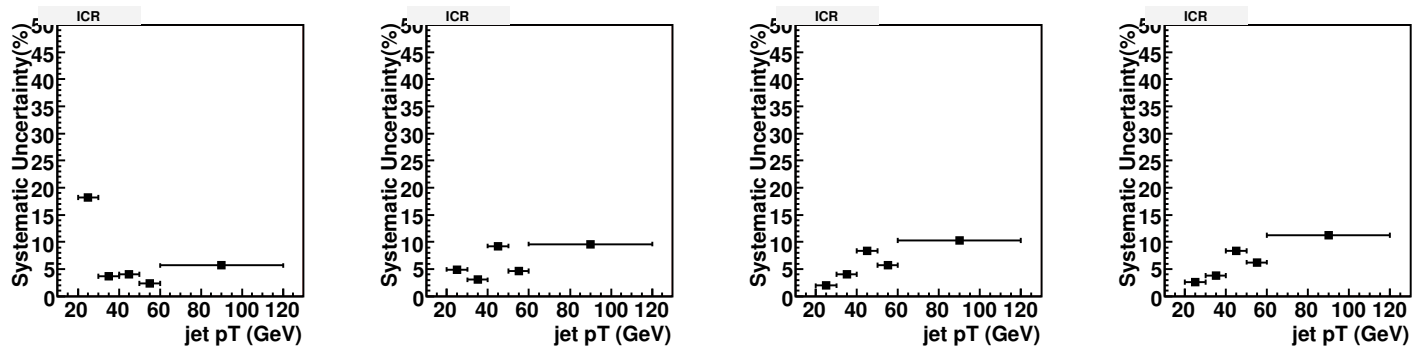

(b) ICR $\eta$ region
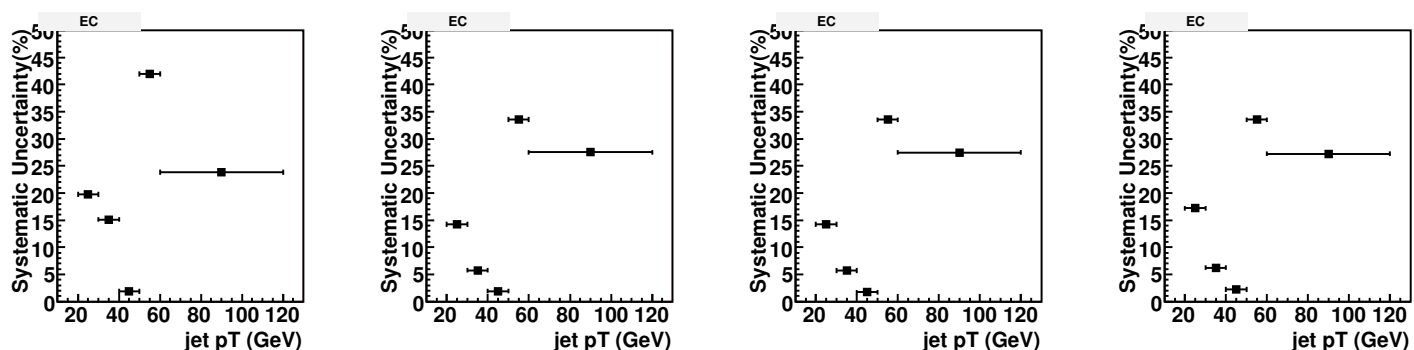

(c) EC $\eta$ region

Figure B.9: Measured systematic uncertainties for each $\eta$ region. From left to right, the plots show the results for Loose muon, Medium muon, Medium3 muon and Tight muon, respectively. 
"modified JLIP (modJLIP)" in this analysis. These two requirements reduce the correlation between the SLTNN tagger and the lifetime tagger, allowing the system8 method to be used to estimate the data tagging efficiency. Figure B.10 shows the distribution of the modJLIP for each muon definition. A modJLIP $<0.1$ cut is required in the system8 method.
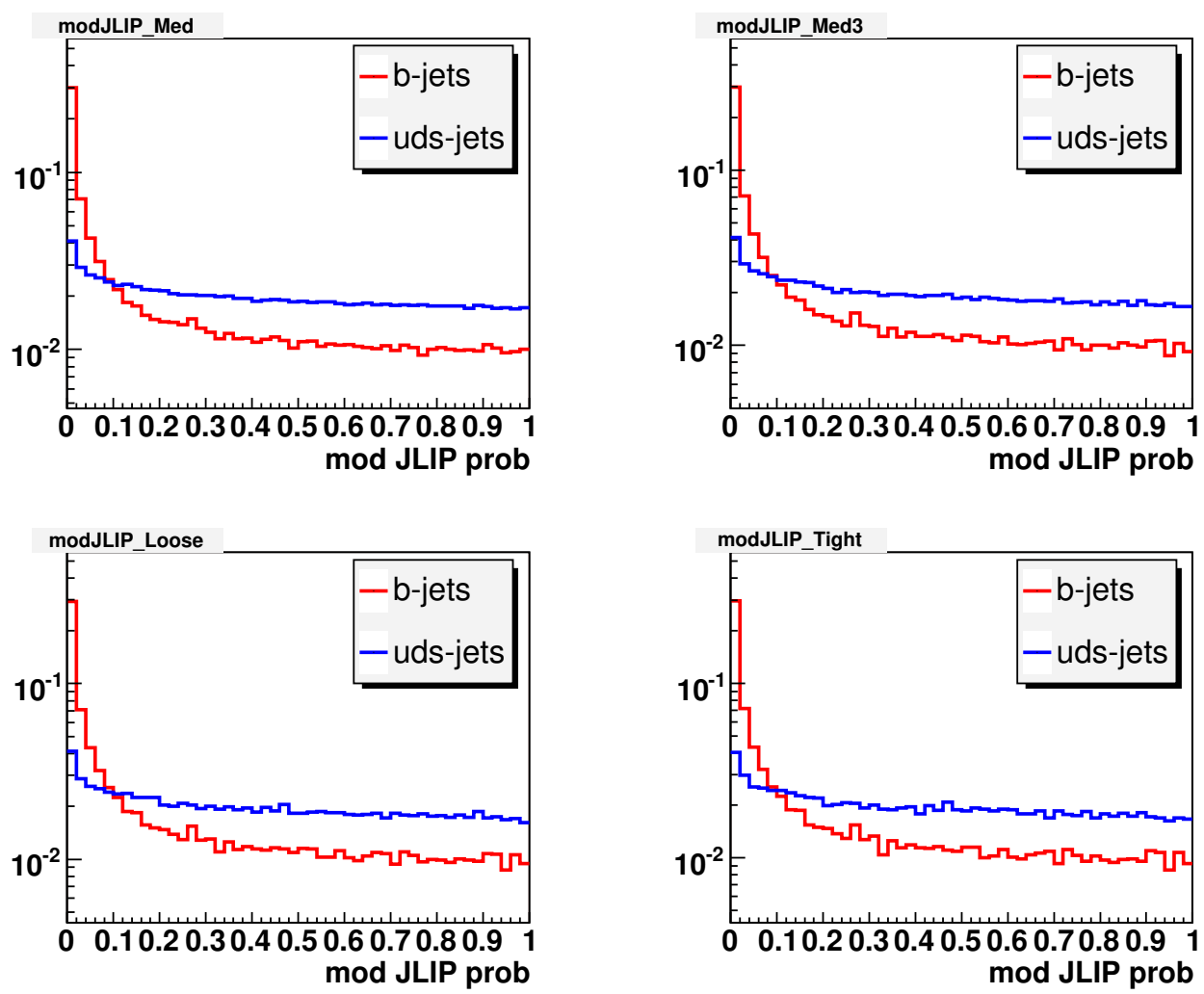

Figure B.10: modJLIP distribution for each muon definition. Loose (top left), Medium (top right), Medium3 (bottom left), and Tight (bottom right). A modJLIP $<0.1$ cut is required in this study.

The muonic and b-enriched samples are used as two different samples in the system8 method. The muonic sample contains events having a jet associated with a muon (Loose, Medium, Medium3, and Tight). The b-enriched sample is a subsample of the muonic sample. An away tag (JLIP < 0.01) is applied to the muonic sample to make the b-enriched sample. An away tag is defined as an event having a back-to-back jet which passes b-tagging criteria (JLIP $<0.01$ in this analysis). 
The system8 equations are defined as follows

$$
\begin{aligned}
n & =n_{b}+n_{c l} \\
p & =p_{b}+p_{c l} \\
n^{\operatorname{modJLIP}} & =\varepsilon_{b}^{\bmod J L I P} \cdot n_{b}+\varepsilon_{c l}^{\bmod J L I P} \cdot n_{c l} \\
p^{\operatorname{modJLIP}} & =\delta \cdot \varepsilon_{b}^{\bmod L L I P} \cdot p_{b}+\gamma \cdot \varepsilon_{c l}^{\bmod L L I P} \cdot p_{c l} \\
n^{S L T N N} & =\varepsilon_{b}^{S L T N N} \cdot n_{b}+\varepsilon_{c l}^{S L T N N} \cdot n_{c l} \\
p^{S L T N N} & =\beta \cdot \varepsilon_{b}^{S L T N N} \cdot p_{b}+\alpha \cdot \varepsilon_{c l}^{S L T N N} \cdot p_{c l} \\
n^{\operatorname{modJLIP}, S L T N N} & =\kappa_{b} \cdot \varepsilon_{b}^{\operatorname{modJLIP}} \cdot \varepsilon_{b}^{S L T N N} \cdot n_{b}+\kappa_{c l} \cdot \varepsilon_{c l}^{\operatorname{modJLIP}} \cdot \varepsilon_{c l}^{S L T N N} \cdot n_{c l} \\
p^{\operatorname{modJLIP}, S L T N N} & =\kappa_{b} \cdot \delta \cdot \beta \cdot \varepsilon_{b}^{\bmod J L I P} \cdot \varepsilon_{b}^{S L T N N} \cdot p_{b}+\kappa_{c l} \cdot \gamma \cdot \alpha \cdot \varepsilon_{c l}^{\operatorname{modJLIP}} \cdot \varepsilon_{c l}^{S L T N N} \cdot p_{c l}
\end{aligned}
$$

where $n(p)$ denotes the number of jets measured in the muonic sample (b-enriched sample). The subscripts $b(c l)$ of $n$ and $p$ indicate the flavor content of the jet is b (udcs). The superscripts indicate the applied tagger. For example, $n^{S L T N N}$ means the number of jets passed by the SLTNN tagger in the muonic sample. $\varepsilon_{b}\left(\varepsilon_{c l}\right)$ denotes the data tagging efficiency estimated for a data sample of b-jets (udcs-jets). In system8, the eight $n$ and $p$ terms with superscripts on the left side are input numbers from the data sample, and $n_{b}, n_{c l}, p_{b}, p_{c l}, \varepsilon_{b}^{\operatorname{modJLIP}}, \varepsilon_{c l}^{\operatorname{modJLIP}}, \varepsilon_{b}^{S L T N N}$ and $\varepsilon_{c l}^{S L T N N}$ on the right side are the 8 unknowns which will be determined by the system 8 calculation. $\varepsilon_{b}^{S L T N N}$ is the data tagging efficiency for the SLTNN tagger determined by the system8 method.

The 6 Greek symbols, $\kappa_{b}, \kappa_{c l}, \beta, \alpha, \delta$, and $\gamma$ are correlation coefficients measured in MC samples. The correlation coefficients would ideally be 1.0, but they are not 1.0 because the samples are not totally uncorrelated. Hence the correlation coefficients are determined in MC samples to account for the correlations. The definitions of the correlation coefficients are as follows:

1. $\kappa_{b}$ : Correlation between the SLTNN tagger and the modJLIP tagger on b-jets

$$
\kappa_{b}=\frac{\varepsilon_{b}^{\bmod J L I P, S L T N N}}{\varepsilon_{b}^{\bmod J L I P} \cdot \varepsilon_{b}^{S L T N N}}
$$


2. $\kappa_{c l}$ : Correlation between the SLTNN tagger and the modJLIP tagger on cl-jets.

$$
\kappa_{c l}=\frac{\varepsilon_{c l}^{\bmod J L I P, S L T N N}}{\varepsilon_{c l}^{\bmod L I P} \cdot \varepsilon_{c l}^{S L T N N}}
$$

3. $\beta$ : Ratio of the b-tagging efficiencies of the SLTNN in the two samples

$$
\beta=\frac{\varepsilon_{b}^{S L T N N} \text { in } \mathrm{b}-\text { enriched sample }}{\varepsilon_{b}^{S L T N N} \text { in muonic sample }}
$$

4. $\alpha$ : Ratio of the cl-tagging efficiencies of the SLTNN in the two samples

$$
\alpha=\frac{\varepsilon_{c l}^{S L T N N} \text { in } \mathrm{b}-\text { enriched sample }}{\varepsilon_{c l}^{S L T N N} \text { in muonic sample }}
$$

5. $\delta$ : Ratio of the b-tagging efficiencies of modJLIP in the two samples

$$
\delta=\frac{\varepsilon_{b}^{\text {modJLIP }} \text { in } \mathrm{b}-\text { enriched sample }}{\varepsilon_{b}^{\text {modJLIP }} \text { in muonic sample }}
$$

6. $\gamma$ : Ratio of the cl-tagging efficiencies of modJLIP in the two samples

$$
\gamma=\frac{\varepsilon_{c l}^{\bmod L I P} \text { in } \mathrm{b}-\text { enriched sample }}{\varepsilon_{c l}^{\operatorname{modJLIP}} \text { in muonic sample }}
$$

Each correlation coefficient for b-jets is measured using the $t \bar{t}$ and $Z \rightarrow b b \rightarrow \mu$ combined MC sample for jet $p_{\mathrm{T}}$ and $\eta$ dependences, respectively. For udcs-jets, it is assumed that c-jets and other lightquark jets have similar correlation coefficients, hence the correlation coefficients are measured in the direct cc and $Z \rightarrow c c \rightarrow \mu$ combined MC sample. The Figures from B.11 B.14 show the results of the correlation coefficient measurement. Table B.6 shows the measured correlation coefficients for the overall sample (without any jet dependence). 

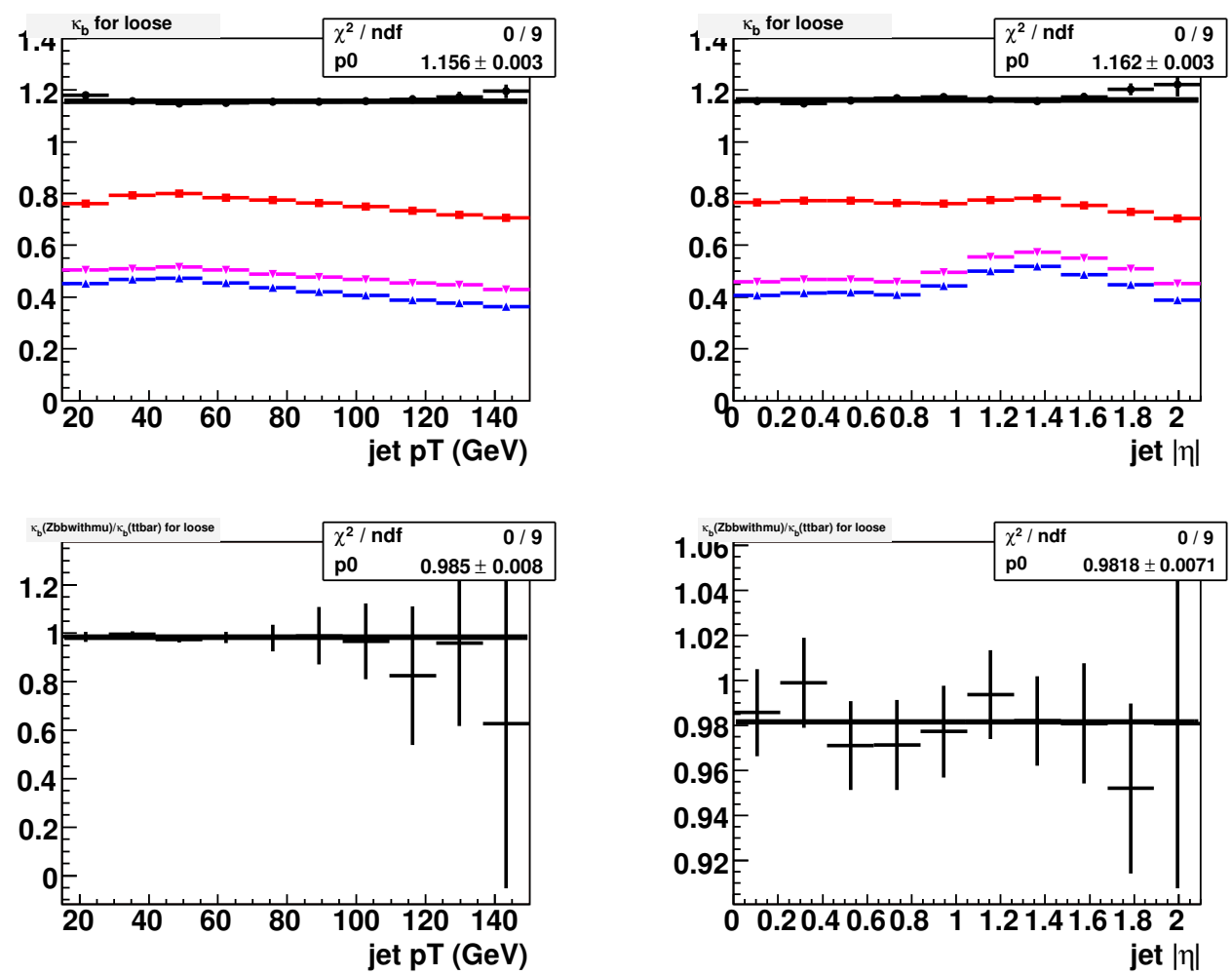

Figure B.11: $\kappa_{b}$ for the Loose Muon and its Tight NN operating point. Top left (right) plot is the coefficient measured in jet $p_{\mathrm{T}}(\eta)$ bins. Bottom left (right) plot is the comparison between coefficients measured in $Z \rightarrow b b \rightarrow \mu$ and $t \bar{t}$ MC samples, respectively. The red line indicates $\varepsilon_{b}^{S L T N N}$, the pink line indicates $\varepsilon_{b}^{\operatorname{modJLIP}}$, the blue line indicates $\varepsilon_{b}^{S L T N N, \bmod J L I P}$. The black line indicates the coefficient estimated by fitting with a zeroth order polynomial function. 

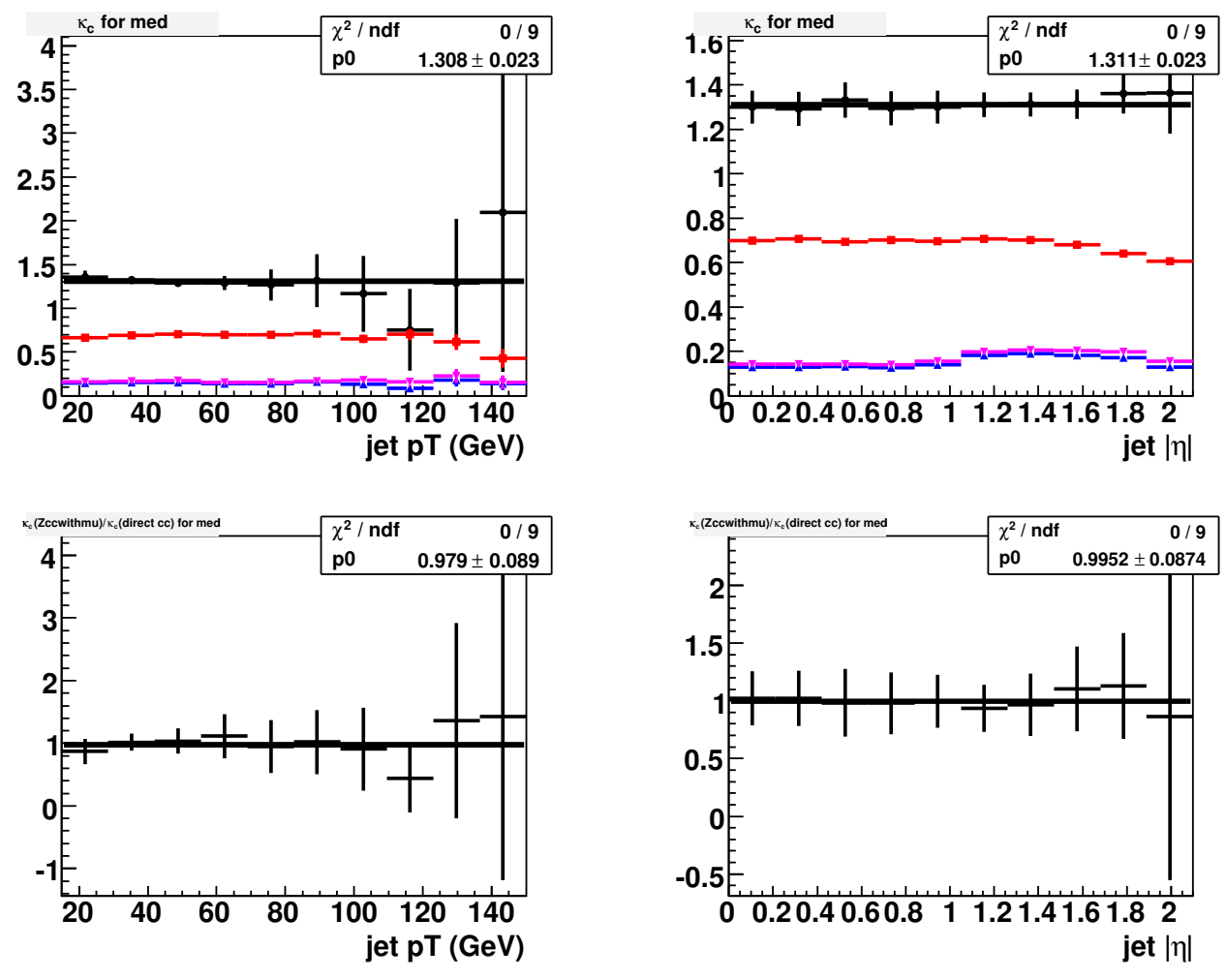

Figure B.12: $\kappa_{c l}$ for the Medium Muon and its Medium NN operating point. Top left (right) plot is the coefficient measured in jet $p_{\mathrm{T}}(\eta)$. Bottom left (right) plot is the comparison between coefficients measured in $Z \rightarrow c c \rightarrow \mu$ and directcc MC samples, respectively. The red line indicates $\varepsilon_{c l}^{S L T N N}$, the pink line indicates $\varepsilon_{c l}^{\text {modJLIP }}$, the blue line indicates $\varepsilon_{c l}^{S L T N N, \text { modJLIP }}$. The black line indicates the coefficient estimated by fitting with a zeroth order polynomial function. 

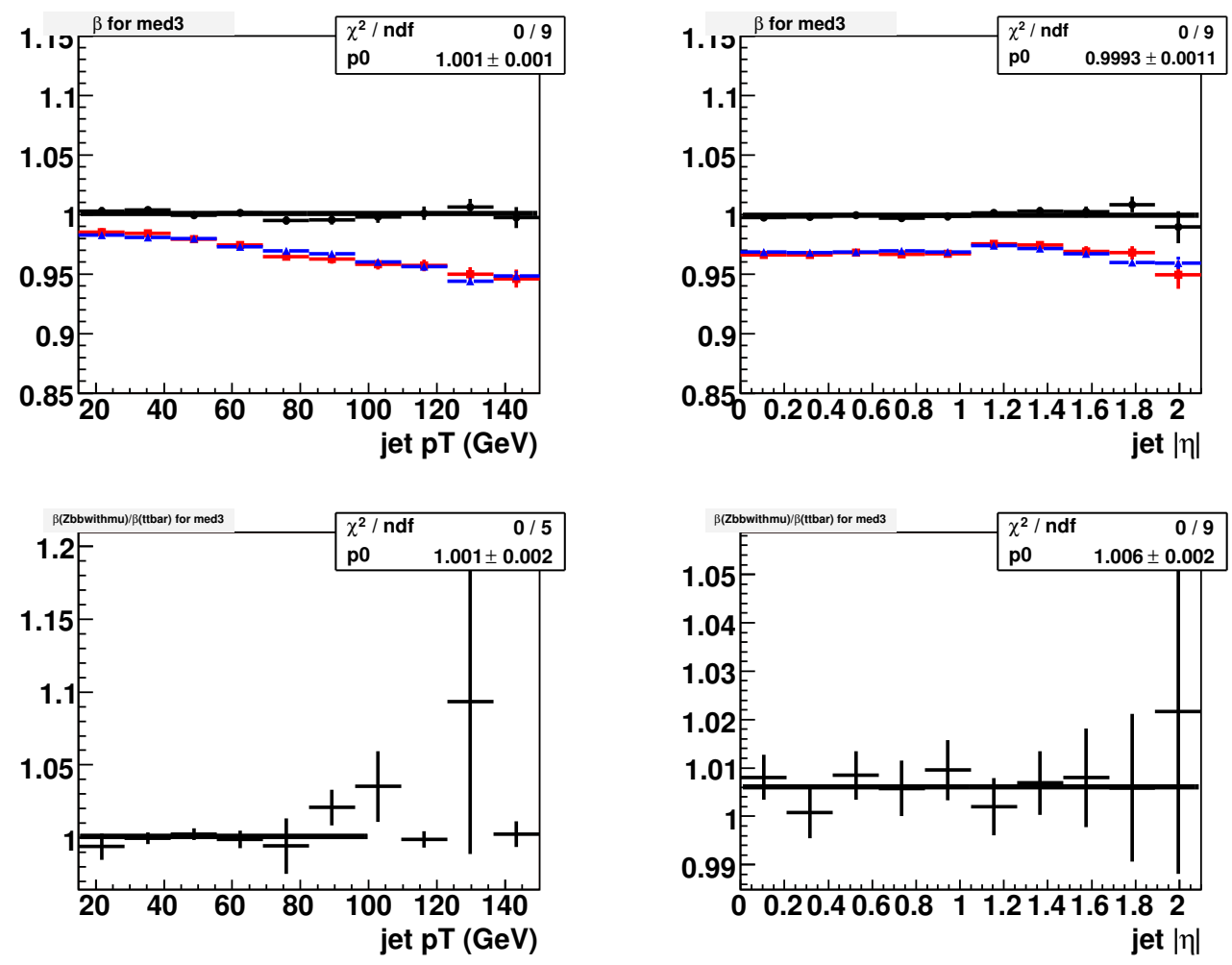

Figure B.13: $\beta$ for the Medium3 Muon and its Loose NN operating point. Top left (right) plot is the coefficient measured in jet $p_{\mathrm{T}}(\eta)$. Bottom left (right) plot is the comparison between coefficients measured in $Z \rightarrow b b \rightarrow \mu$ and $t \bar{t}$ MC samples, respectively. The red (blue) line indicates $\varepsilon^{S L T N N}$ meaured in muonic (b-enriched) sample. The black line indicates the coefficient estimated by fitting with a zeroth order polynomial function. 

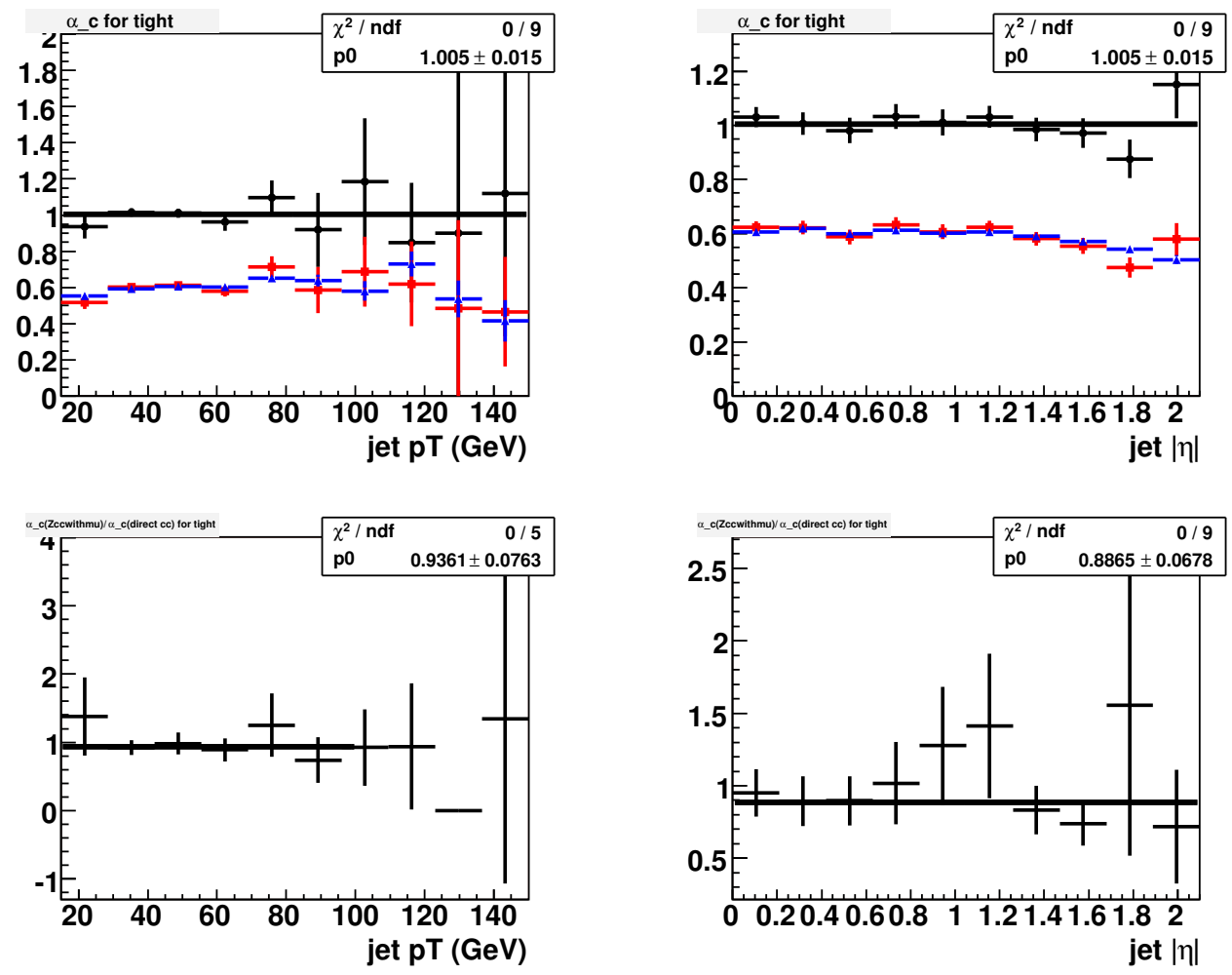

Figure B.14: $\alpha$ for the Tight Muon and its Tight NN operating point. Top left (right) plot is the coefficient measured in jet $p_{\mathrm{T}}(\eta)$. Bottom left (right) plot is the comparison between coefficients measured in $Z \rightarrow c c \rightarrow \mu$ and directcc MC samples, respectively. The red (blue) line indicates $\varepsilon^{S L T N N}$ meaured in muonic (b-enriched) sample. The black line indicates the coefficient estimated by fitting with a zeroth order polynomial function. 


\begin{tabular}{|c|c|c|c|c|}
\hline \hline Muon selection & Operating point & $\kappa_{b}$ & $\kappa_{c l}$ & $\beta$ \\
\hline \multirow{3}{*}{ Loose } & Loose & $1.090 \pm 0.003$ & $1.469 \pm 0.027$ & $1.001 \pm 0.002$ \\
& Medium & $1.110 \pm 0.003$ & $1.579 \pm 0.030$ & $0.999 \pm 0.002$ \\
& Tight & $1.162 \pm 0.003$ & $1.789 \pm 0.038$ & $0.998 \pm 0.003$ \\
\hline & Loose & $1.038 \pm 0.003$ & $1.185 \pm 0.019$ & $1.000 \pm 0.001$ \\
& Medium & $1.055 \pm 0.003$ & $1.310 \pm 0.023$ & $0.999 \pm 0.002$ \\
& Tight & $1.105 \pm 0.003$ & $1.600 \pm 0.032$ & $1.000 \pm 0.002$ \\
\hline \multirow{3}{*}{ Medium3 } & Loose & $1.022 \pm 0.003$ & $1.081 \pm 0.016$ & $0.999 \pm 0.001$ \\
& Medium & $1.038 \pm 0.003$ & $1.206 \pm 0.021$ & $0.998 \pm 0.001$ \\
& Tight & $1.087 \pm 0.003$ & $1.512 \pm 0.030$ & $0.998 \pm 0.002$ \\
\hline \multirow{3}{*}{ Tight } & Loose & $1.011 \pm 0.003$ & $1.044 \pm 0.014$ & $0.999 \pm 0.001$ \\
& Medium & $1.026 \pm 0.003$ & $1.152 \pm 0.019$ & $1.000 \pm 0.001$ \\
& Tight & $1.070 \pm 0.003$ & $1.440 \pm 0.029$ & $0.998 \pm 0.002$ \\
\hline \hline Muon selection & Operating point & $\alpha$ & $\delta$ & \\
\hline & Loose & $1.004 \pm 0.014$ & $0.998 \pm 0.005$ & $0.993 \pm 0.041$ \\
& Medium & $0.992 \pm 0.017$ & $0.997 \pm 0.006$ & $0.985 \pm 0.044$ \\
& Tight & $1.018 \pm 0.022$ & $0.995 \pm 0.006$ & $1.023 \pm 0.047$ \\
\hline \multirow{3}{*}{ Medium } & Loose & $0.993 \pm 0.009$ & $0.998 \pm 0.006$ & $1.011 \pm 0.040$ \\
& Medium & $1.014 \pm 0.011$ & $0.999 \pm 0.006$ & $1.011 \pm 0.041$ \\
& Tight & $1.010 \pm 0.018$ & $0.999 \pm 0.006$ & $1.022 \pm 0.044$ \\
\hline \multirow{3}{*}{ Medium3 } & Loose & $0.998 \pm 0.006$ & $0.998 \pm 0.006$ & $1.015 \pm 0.040$ \\
& Medium & $1.004 \pm 0.009$ & $0.997 \pm 0.006$ & $1.007 \pm 0.041$ \\
& Tight & $1.001 \pm 0.017$ & $0.999 \pm 0.006$ & $1.012 \pm 0.044$ \\
\hline & Loose & $1.004 \pm 0.004$ & $1.000 \pm 0.006$ & $1.008 \pm 0.041$ \\
& Medium & $0.999 \pm 0.008$ & $1.000 \pm 0.006$ & $1.009 \pm 0.042$ \\
& Tight & $1.008 \pm 0.015$ & $1.000 \pm 0.007$ & $1.019 \pm 0.044$ \\
\hline \hline
\end{tabular}

Table B.6: Summary of correlation coefficients with statistical uncertainties.

\section{B.3.3 The Results for Tagging Efficiencies}

The b-tagging efficiency is measured in the overall MUinclusive sample and the TMinuit fitting program [75] is used to solve the system 8 equations. The fit is carried out on the entire sample and in jet $p_{\mathrm{T}}$ and $\eta$ bins to create a profile over the jet $p_{\mathrm{T}}$ and $\eta$ phase space variables. Table B.7 shows the results for b-tagging efficiencies measured for the data sample.

There are discrepancies in the measured tagging efficiencies between the $\mathrm{MC}$ and data samples. Therefore, a scale factor is calculated from the ratio of tagging efficiencies, parameterized by jet $p_{\mathrm{T}}$ and $\eta$. To create the $2 \mathrm{D}$ parameterization, it is assumed that the efficiency can be factorized into $p_{\mathrm{T}}$ and $\eta$ components. The $2 \mathrm{D}$ parameterization is given by the product of the two projections 


\begin{tabular}{|c|c|c|}
\hline \hline Muon selection & Operating point & b-tagging efficiency \\
\hline \multirow{3}{*}{ Loose } & Loose & $0.876 \pm 0.001$ \\
& Medium & $0.848 \pm 0.001$ \\
& Tight & $0.773 \pm 0.001$ \\
\hline \multirow{3}{*}{ Medium } & Loose & $0.950 \pm 0.000$ \\
& Medium & $0.926 \pm 0.001$ \\
& Tight & $0.857 \pm 0.001$ \\
\hline \multirow{3}{*}{ Medium3 } & Loose & $0.973 \pm 0.000$ \\
& Medium & $0.951 \pm 0.000$ \\
& Tight & $0.886 \pm 0.001$ \\
\hline \multirow{2}{*}{ Tight } & Loose & $0.987 \pm 0.000$ \\
& Medium & $0.965 \pm 0.000$ \\
& Tight & $0.905 \pm 0.001$ \\
\hline \hline
\end{tabular}

Table B.7: b-tagging efficiency measured by the system8 method for the MUinclusive sample for each muon definition and its $\mathrm{NN}$ operating points.

scaled by the total efficiency of the sample:

$$
\begin{aligned}
\varepsilon\left(p_{\mathrm{T}}, \eta\right) & =\frac{1}{\varepsilon^{A l l}} \cdot \varepsilon\left(p_{\mathrm{T}}\right) \cdot \varepsilon(\eta) \\
& =\frac{1}{\varepsilon^{A l l}} \cdot\left(a+b \cdot p_{\mathrm{T}}+c \cdot{p_{\mathrm{T}}}^{2}+d \cdot{p_{\mathrm{T}}}^{3}\right) \cdot\left(e+f \cdot \eta+g \cdot \eta^{2}+h \cdot \eta^{3}\right)
\end{aligned}
$$

where $a \sim h$ are constants to be determined by fitting and the fitting funtion is a third order polynomial. The scale factor is calculated by dividing the data tagging efficiency by the MC tagging efficiency. Figure B.15 shows the tagging efficiencies in both $\mathrm{MC}$ and data samples as a function of jet $p_{\mathrm{T}}$ and $\eta$ and the scale factor between them.

The inclusive b-tagging efficiency in the data sample can be computed by taking the product of the inclusive b-tagging efficiency in MC sample and the scale factor. It is called the "Tag Rate Function" $\left(T R F_{b}\right)$ for b-jets, and the same procedure is applied to c-jets to create $T R F_{c}$. Figure B.16 shows $T R F_{b}$ and $T R F_{c}$ for the medium3 muon, Medium operating point.

\section{B.3.4 Systematic Uncertainties}

There are two sources of systematic unceratinties for the scale factor and $T R F$. The systematic unceratinty caused by the system 8 method can be determined by varying by $\pm 1 \sigma$ the statistical 

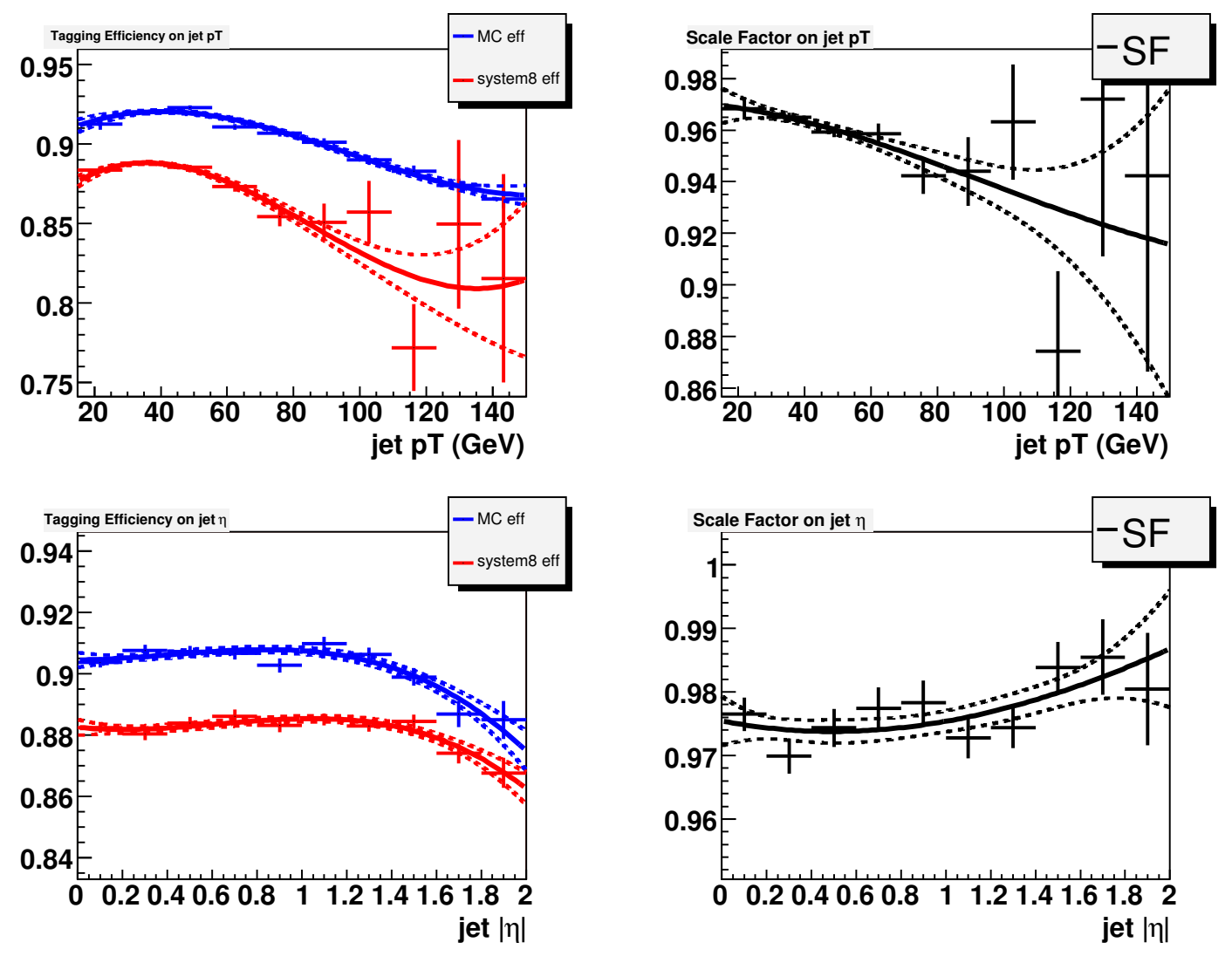

Figure B.15: b-tagging efficiency and scale factor for the Tight muon, Tight NN operating point. Left plots are the b-tagging efficiency of data (red) and MC sample (blue). Right plots are the scale factors for jet $p_{\mathrm{T}}$ (top) and $\eta$ (bottom). The dashed lines denote estimated statistical uncertainties. 

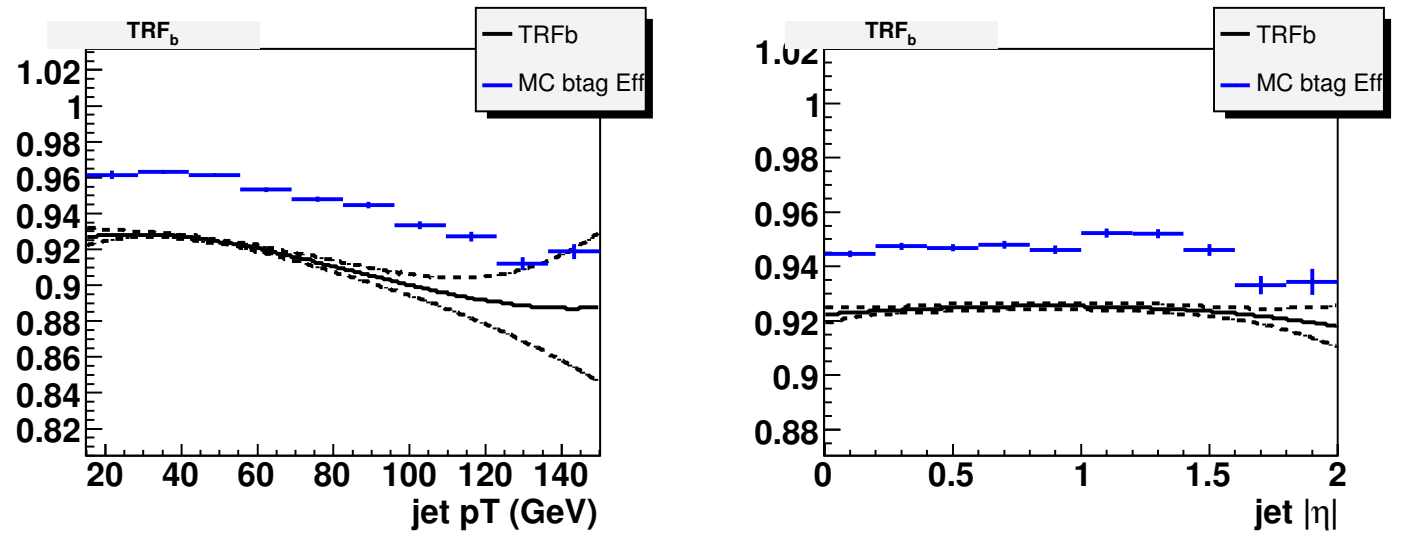

(a) $T R F_{b}$
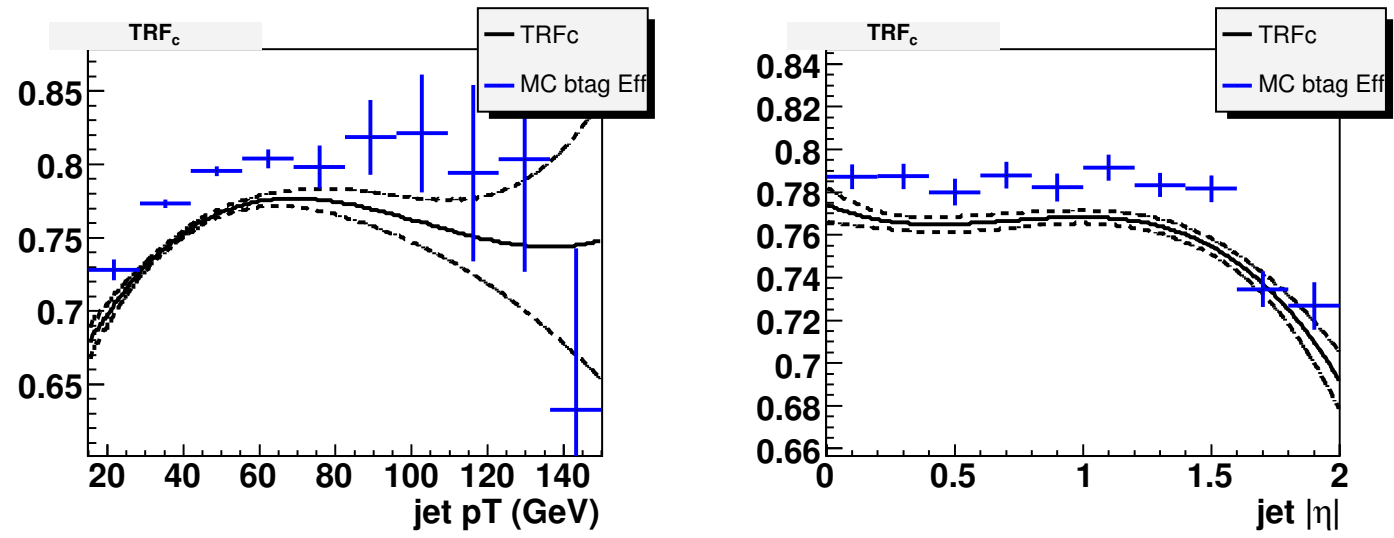

(b) $T R F_{c}$

Figure B.16: $T R F_{b}$ (top) and $T R F_{c}$ (bottom) measured in the medium3 muon, medium NN operating point. Left plots are for the jet $p_{\mathrm{T}}$ distribution and right plots are for the jet $\eta$ distribution. The blue line is the MC b-tagging efficiency and the black line is the TRF. The dashed lines denotes estimated statistical uncertainties. 
error of individual correlation coefficients. The systematic uncertainty caused by the MC sample dependence in the system 8 method can be estimated by a closure test, which is the difference between direct tagging and multiplying the untagged sample by the $T R F$. The closure test is performed as a function of jet $p_{\mathrm{T}}$ in 3 jet $\eta$ regions $(\mathrm{CC}, \mathrm{ICR}$ and $\mathrm{EC}$ ) individually, where CC region covers $0<|\eta|<1.2$, ICR covers $1.2<|\eta|<1.8$ and EC covers $1.8<|\eta|<2.5$. The closure test is performed for all of the MC samples and the combined sample which are used in system8 method. Then, the largest uncertainty among them is chosen as the uncertainty of the closure test. Using the estimated systematic uncertainties from the two sources, the total systematic uncertainties for the scale factor and $T R F$ are calculated by the following formula:

$$
\begin{aligned}
\delta_{S F} & =\sqrt{\left(\delta_{\text {sys } 8}\right)^{2}+\left(\delta_{\text {selected }}^{\text {closure }}\right)^{2}} \\
\delta_{T R F_{b}} & =\sqrt{\left(\delta_{S F}\right)^{2}+\left(\delta_{b \text { combined }}^{\text {closure }}\right)^{2}} \\
\delta_{T R F_{c}} & =\sqrt{\left(\delta_{S F}\right)^{2}+\left(\delta_{\text {c closure }}^{\text {combined }}\right)^{2}} .
\end{aligned}
$$

Table B.8 shows the estimated systematic uncertainties.

\begin{tabular}{|c|c|c|c|c|c|c|c|}
\hline \hline \multirow{2}{*}{ Muon Def. } & \multirow{2}{*}{ NN Operating Point } & \multicolumn{5}{|c|}{ Systematic Uncertainty } \\
\cline { 3 - 7 } & & System8 & Closure (b) & Closure (c) & $S F$ & $T R F_{b}$ & $T R F_{c}$ \\
\hline \multirow{3}{*}{ Loose } & Loose & $0.49 \%$ & $2.87 \%$ & $6.12 \%$ & $2.91 \%$ & $2.97 \%$ & $6.77 \%$ \\
& Medium & $0.59 \%$ & $3.22 \%$ & $6.52 \%$ & $3.28 \%$ & $3.35 \%$ & $7.30 \%$ \\
& Tight & $0.89 \%$ & $3.86 \%$ & $8.05 \%$ & $3.96 \%$ & $4.03 \%$ & $8.97 \%$ \\
\hline \multirow{3}{*}{ Medium } & Loose & $0.37 \%$ & $1.37 \%$ & $1.93 \%$ & $1.41 \%$ & $1.55 \%$ & $2.39 \%$ \\
& Medium & $0.42 \%$ & $1.67 \%$ & $2.09 \%$ & $1.72 \%$ & $1.85 \%$ & $2.71 \%$ \\
& Tight & $0.48 \%$ & $2.45 \%$ & $2.79 \%$ & $2.52 \%$ & $2.67 \%$ & $3.76 \%$ \\
\hline \multirow{3}{*}{ Medium3 } & Loose & $0.33 \%$ & $0.68 \%$ & $0.93 \%$ & $0.76 \%$ & $0.80 \%$ & $1.20 \%$ \\
& Medium & $0.37 \%$ & $1.06 \%$ & $1.08 \%$ & $1.12 \%$ & $1.18 \%$ & $1.56 \%$ \\
& Tight & $0.53 \%$ & $1.40 \%$ & $1.36 \%$ & $1.50 \%$ & $1.57 \%$ & $2.03 \%$ \\
\hline \multirow{2}{*}{ Tight } & Loose & $0.32 \%$ & $0.27 \%$ & $0.38 \%$ & $0.41 \%$ & $0.45 \%$ & $0.56 \%$ \\
& Medium & $0.35 \%$ & $0.57 \%$ & $0.64 \%$ & $0.67 \%$ & $0.69 \%$ & $0.93 \%$ \\
& Tight & $0.50 \%$ & $1.20 \%$ & $0.17 \%$ & $1.30 \%$ & $1.36 \%$ & $1.31 \%$ \\
\hline \hline
\end{tabular}

Table B.8: Estimated systematic uncertainties for $S F, T R F_{b}$, and $T R F_{c}$. The systematic uncertainties estimated by the system 8 method and closure tests are also shown in this table. 


\section{B.4 Fake Rate}

The negative tag rate (NTR) method is used to measure the fake rate for light-quark jets. The formula for the NTR is as follows:

$$
\varepsilon_{\text {light }}=\varepsilon_{\text {data }}^{-} \cdot F_{h f} \cdot F_{l l}
$$

where

$$
\begin{aligned}
& \varepsilon_{\text {data }}^{-} \text {is the negative tag rate measured in QCD data } \\
& F_{h f}=\frac{\varepsilon_{Q C D l i g h t}^{-}}{\varepsilon_{Q C \text { Dall }}^{-}} \text {is the fraction of light-quark jets among all negative tagged jets in the } \\
& \mathrm{MC} \\
& F_{l l}=\frac{\varepsilon_{Q C D l i g h t}^{+}}{\varepsilon_{Q C D l i g h t}^{-}} \text {is the ratio of positive tagged jets to negative tagged jets from light } \\
& \text { quarks in the MC. It is sensitive to long lived hadron decays in light quark jets. }
\end{aligned}
$$

The two scale factor, $F_{h f}$ and $F_{l l}$ are measured in the QCD and $\gamma+$ jets combined MC sample and $\varepsilon_{\text {data }}^{-}$is measured in the QCD data sample.

To evaluate the NTR, muon IP significance is used. The muon IP significance is described in Table B.4 and shown in Figure B.1. There are more jets with positive muon IP significance than with negative significance. The NTR is evaluated by using this difference. Each jet $\eta$ region has a different distribution for the NTR, therefore the NTR is measured for CC, ICR and EC individually with a jet $p_{\mathrm{T}}$ dependence.

The fake rate estimated in the overall QCD data sample is shown in Table B.9. Figure B.17 shows the results for $F_{h f}$ (top left), $F_{l l}$ (top right), $\varepsilon_{\text {data }}^{-}$(bottom left), and $\varepsilon_{\text {light }}$ (bottom right) in the $\mathrm{CC}$ region for the loose muon, tight $\mathrm{NN}$ operating point as a function of jet $p_{\mathrm{T}}$.

Systematic uncertainties in the fake rate are measured by the closure test used for the systematic uncertainty estimation of the $T R F$. A second source of systematic uncertainty is estimated by varying the b-fraction and c-fraction by $\pm 20 \%$ in the QCD and $\gamma+$ jets combined MC sample. Using the remeasured scale factor $F_{h f}$ and $F_{l l}$, the fake rates are recalculated, and the differences are taken as the systematic uncertainties. The total systematic uncertainty is calculated by the quadrature 


\begin{tabular}{|c|c|c|c|c|}
\hline \hline \multirow{2}{*}{ Muon selection } & \multirow{2}{*}{ Operating point } & \multicolumn{3}{|c|}{ Fake Rate (\%) } \\
\cline { 3 - 5 } & & CC & ICR & EC \\
\hline \multirow{4}{*}{ Loose } & Nocut & $0.914 \pm 0.005$ & $0.488 \pm 0.003$ & $0.200 \pm 0.002$ \\
& Loose & $0.090 \pm 0.003$ & $0.046 \pm 0.002$ & $0.013 \pm 0.001$ \\
& Medium & $0.051 \pm 0.003$ & $0.027 \pm 0.001$ & $0.007 \pm 0.000$ \\
& Tight & $0.011 \pm 0.001$ & $0.006 \pm 0.001$ & $0.001 \pm 0.000$ \\
\hline \multirow{4}{*}{ Medium } & Nocut & $0.225 \pm 0.003$ & $0.190 \pm 0.003$ & $0.039 \pm 0.001$ \\
& Loose & $0.080 \pm 0.002$ & $0.068 \pm 0.002$ & $0.014 \pm 0.000$ \\
& Medium & $0.056 \pm 0.002$ & $0.047 \pm 0.002$ & $0.009 \pm 0.000$ \\
& Tight & $0.017 \pm 0.001$ & $0.014 \pm 0.001$ & $0.003 \pm 0.000$ \\
\hline \multirow{3}{*}{ Tight } & Nocut & $0.130 \pm 0.003$ & $0.132 \pm 0.003$ & $0.034 \pm 0.001$ \\
& Loose & $0.080 \pm 0.002$ & $0.082 \pm 0.002$ & $0.020 \pm 0.001$ \\
& Medium & $0.054 \pm 0.002$ & $0.054 \pm 0.002$ & $0.013 \pm 0.000$ \\
& Tight & $0.015 \pm 0.001$ & $0.014 \pm 0.001$ & $0.003 \pm 0.000$ \\
\hline \hline & Nocut & $0.099 \pm 0.002$ & $0.124 \pm 0.003$ & $0.032 \pm 0.001$ \\
& Loose & $0.073 \pm 0.002$ & $0.091 \pm 0.003$ & $0.023 \pm 0.001$ \\
& Medium & $0.047 \pm 0.002$ & $0.056 \pm 0.002$ & $0.014 \pm 0.001$ \\
& Tight & $0.017 \pm 0.001$ & $0.019 \pm 0.001$ & $0.004 \pm 0.000$ \\
\hline \hline
\end{tabular}

Table B.9: Fake rate measured in the overall QCD data sample (without any jet dependence).
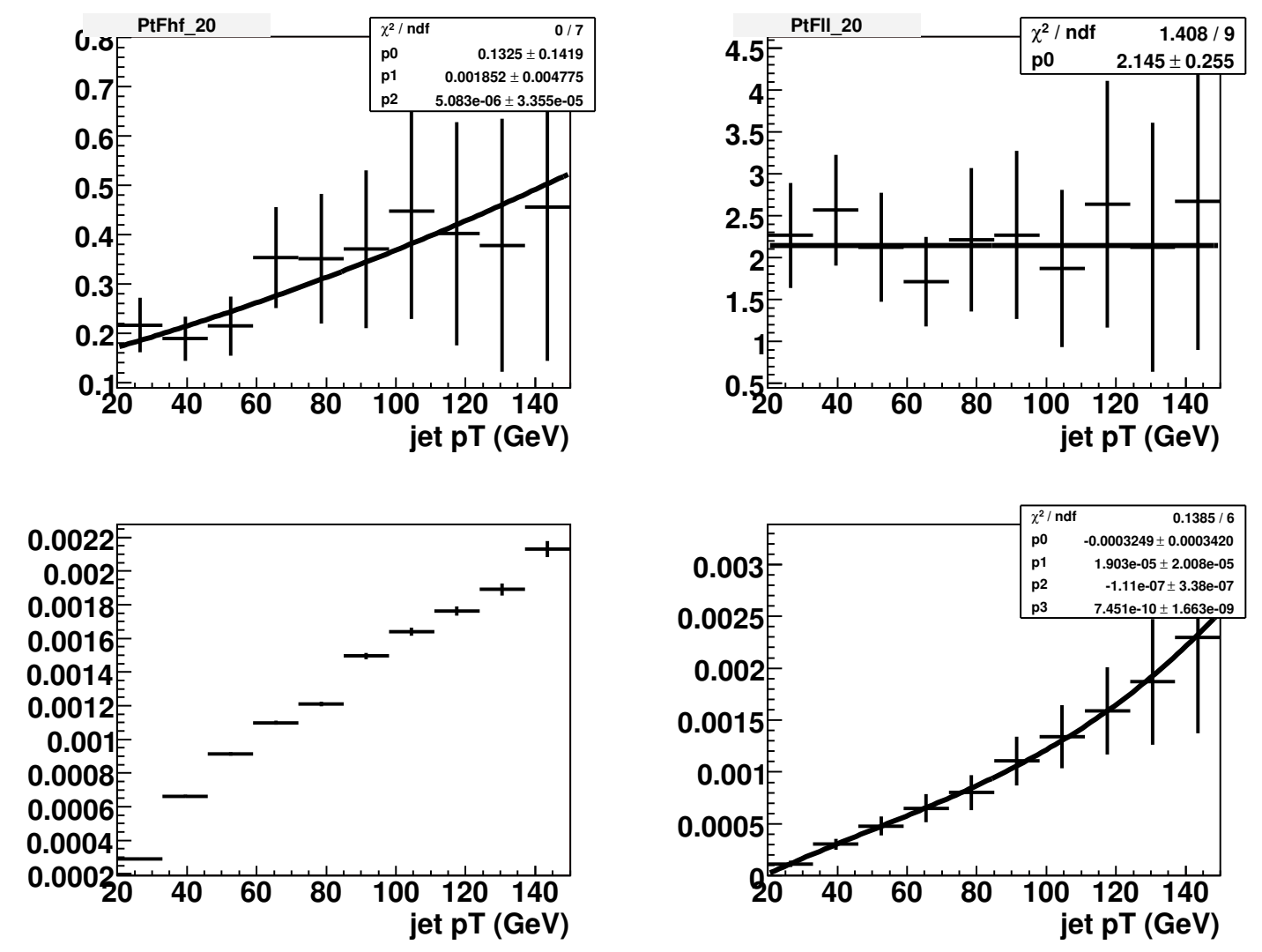

Figure B.17: The fake rate as fuction of jet $p_{\mathrm{T}}$ measured in the loose muon, tight NN operating point, CC region. $F_{h f}$ (top left), $F_{l l}$ (top right), $\varepsilon_{\text {data }}^{-}$(bottom left), and $\varepsilon_{\text {light }}$ (bottom right). 


\begin{tabular}{|c|c|c|c|c|}
\hline \hline \multirow{2}{*}{ Muon selection } & \multirow{2}{*}{ Operating point } & \multicolumn{3}{|c|}{ Systematic Uncertainty } \\
\cline { 3 - 5 } & & CC & ICR & EC \\
\hline \multirow{3}{*}{ Loose } & NoCut & $2.20 \%$ & $2.33 \%$ & $2.33 \%$ \\
& Loose & $6.72 \%$ & $6.79 \%$ & $6.79 \%$ \\
& Medium & $7.77 \%$ & $7.80 \%$ & $7.93 \%$ \\
& Tight & $10.12 \%$ & $10.16 \%$ & $10.22 \%$ \\
\hline \multirow{4}{*}{ Medium } & NoCut & $3.99 \%$ & $4.04 \%$ & $4.07 \%$ \\
& Loose & $6.52 \%$ & $6.54 \%$ & $6.55 \%$ \\
& Medium & $7.39 \%$ & $7.42 \%$ & $7.43 \%$ \\
& Tight & $9.72 \%$ & $9.75 \%$ & $9.80 \%$ \\
\hline \multirow{3}{*}{ Medium3 } & NoCut & $5.24 \%$ & $5.29 \%$ & $5.28 \%$ \\
& Loose & $6.50 \%$ & $6.56 \%$ & $6.56 \%$ \\
& Medium & $7.37 \%$ & $7.42 \%$ & $7.42 \%$ \\
& Tight & $9.69 \%$ & $9.70 \%$ & $9.75 \%$ \\
\hline & NoCut & $5.71 \%$ & $5.77 \%$ & $5.77 \%$ \\
& Loose & $6.41 \%$ & $6.48 \%$ & $6.51 \%$ \\
& Medium & $7.31 \%$ & $7.38 \%$ & $7.38 \%$ \\
& Tight & $9.54 \%$ & $9.53 \%$ & $9.53 \%$ \\
\hline \hline
\end{tabular}

Table B.10: Systematic uncertainty in the fake rate for CC, ICR and EC regions.

sum of all systematic uncertainties. Table B.10 shows the estimated systematic uncertainties for the fake rate.

\section{B.5 $t \bar{t}$ Cross Section Measurement Using the SLTNN Tag- ging}

The newly developed b-tagging algorithm, the SLTNN tagger, is applied to the $t \bar{t}$ pair production cross section measurement. The procedure for the $t \bar{t}$ cross section measurement (discussed in Chapters $4 \sim 6$ ) is applied to this analysis with the SLTNN tagger. In this section, the results of the analysis using the SLTNN tagger are described.

\section{B.5.1 b-tagging Optimization}

There are 16 operating points for the SLTNN tagger including the pure SLT tagger (called "NoCut"). To decide on one of the 16 operating points, the same procedure of b-tagging optimization described in Section 4.4 is applied. Namely, the signal and background ratio, $S / \sqrt{S+B}$ is calculated for 
each operating point and the operating point having largest ratio is selected. Table B.11 shows the results of $S / \sqrt{S+B}$ ratio for each operating point. This optimization is performed for the combined $e+\geq 3$ jets and $\mu+\geq 3$ jets channels. The best operating point is the loose NN operating point for the loose muon definition.

\begin{tabular}{|c|c|c|c|c|}
\hline \hline Muon selection & Operating point & $S$ & $B$ & $S / \sqrt{S+B}$ \\
\hline \multirow{4}{*}{ Loose } & NoCut & 106.68 & 113.78 & 7.18 \\
& Loose & 97.20 & 51.69 & 7.97 \\
& Medium & 81.11 & 40.49 & 7.36 \\
& Tight & 86.79 & 36.52 & 7.82 \\
\hline \multirow{4}{*}{ Medium } & NoCut & 80.34 & 61.19 & 6.75 \\
& Loose & 70.50 & 39.36 & 6.73 \\
& Medium & 58.48 & 31.62 & 6.16 \\
& Tight & 63.06 & 27.59 & 6.62 \\
\hline \multirow{3}{*}{ Medium3 } & NoCut & 74.06 & 49.47 & 6.66 \\
& Loose & 66.80 & 39.02 & 6.49 \\
& Medium & 64.84 & 34.81 & 6.50 \\
& Tight & 56.09 & 25.01 & 6.23 \\
\hline \multirow{5}{*}{ Tight } & NoCut & 67.63 & 43.44 & 6.42 \\
& Loose & 67.25 & 40.12 & 6.49 \\
& Medium & 67.00 & 36.26 & 6.59 \\
& Tight & 55.36 & 24.93 & 6.18 \\
\hline \hline
\end{tabular}

Table B.11: The results of $S / \sqrt{S+B}$ ratio for each operating point. The best operating point is the Loose NN operating point with the loose muon definition.

\section{B.5.2 Control Plots}

In this section, the control plots for the main kinematic distributions (lepton $p_{\mathrm{T}}, \mathbb{E}_{T}$, leading jet $p_{\mathrm{T}}$, and $\mathrm{W}$ transverse momentum) are shown in Figures B.18 B.25. The number of events for two or more btags are very small because the efficiency of the SLTNN tagger is low $(\sim 6 \%)$. Therefore the exactly one tagged sample and the two or more tagged sample are combined in this analysis (called the single tagged sample). The control plots are shown only for the single tagged sample in this section because the control plots for before tagging are the same as the figures in Chapter 5 and Appendix C. Table B.12 and B.13 show the summaries of event yields after SLTNN tagging. 

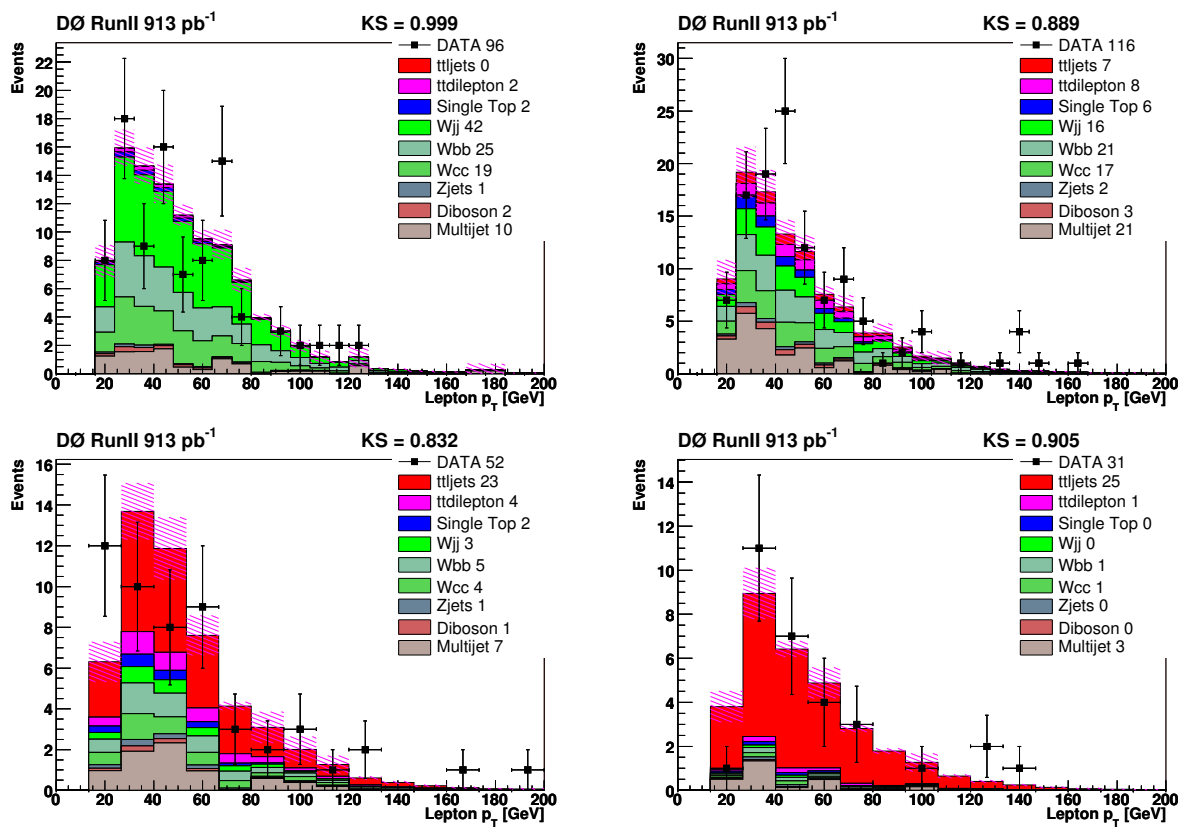

Figure B.18: Electron $p_{\mathrm{T}}$ distributions for single tagged sample in the $e+$ jets channel. The plots show the data for different jet multiplicities: $=1$ jet (top left), $=2$ jets (top right), $=3$ jets (bottom left), and $\geq 4$ jets (bottom right).
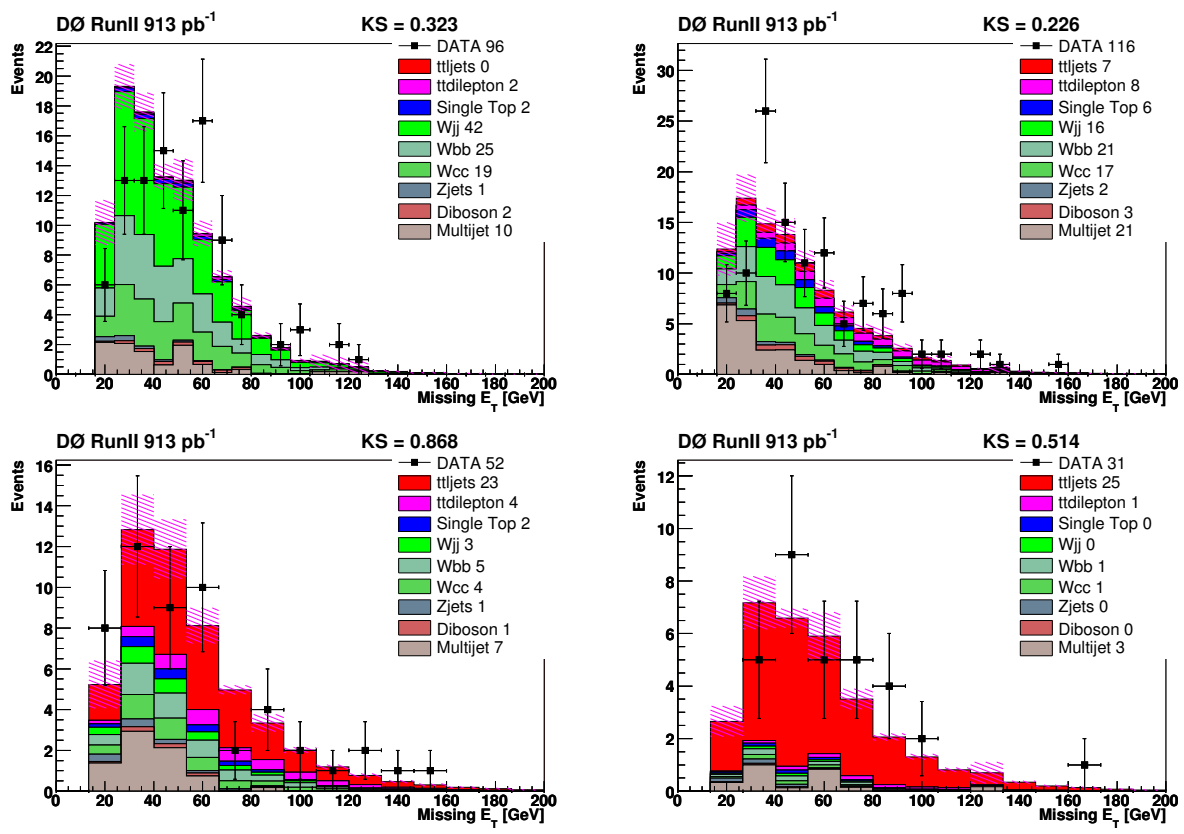

Figure B.19: $\mathbb{E}_{T}$ distributions for single tagged sample in the $e+$ jets channel. The plots show the data for different jet multiplicities: $=1$ jet (top left), $=2$ jets (top right), $=3$ jets (bottom left), and $\geq 4$ jets (bottom right). 

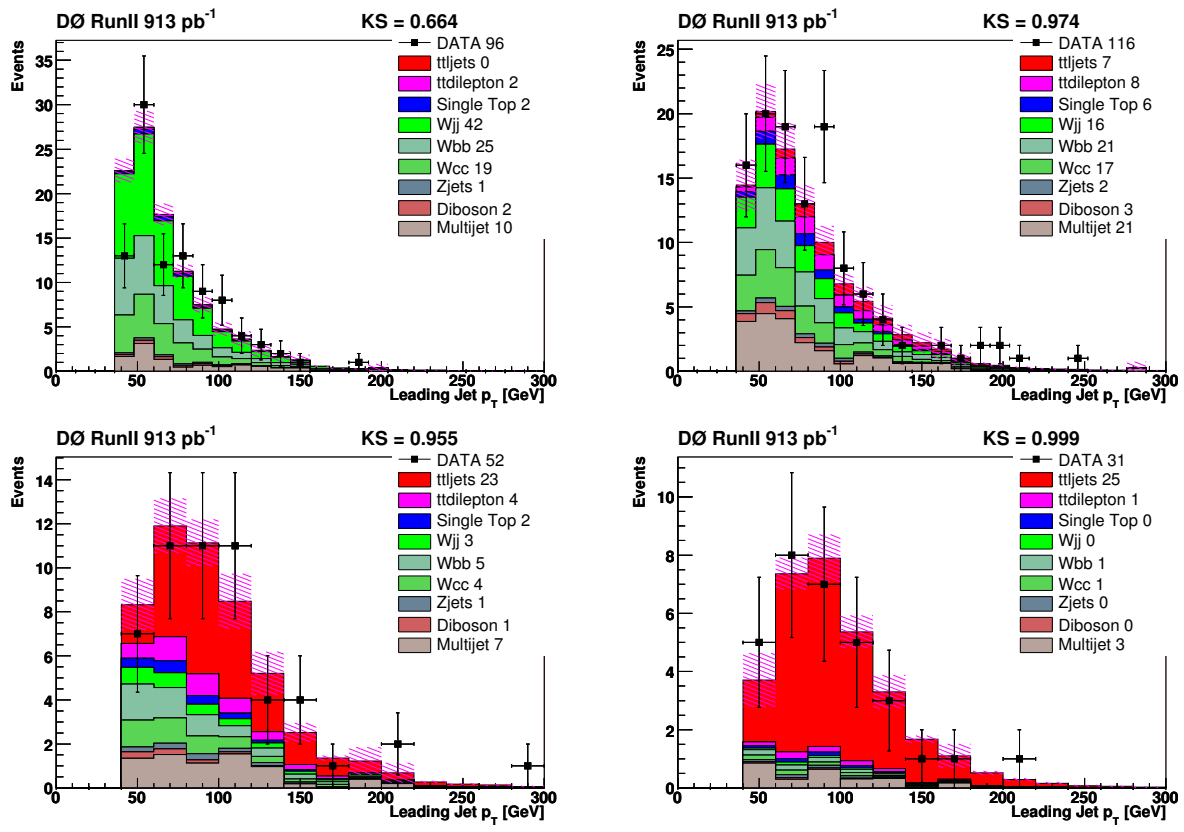

Figure B.20: Leading jet $p_{\mathrm{T}}$ distributions for single tagged sample in the $e+$ jets channel. The plots show the data for different jet multiplicities: $=1$ jet (top left), $=2$ jets (top right), $=3$ jets (bottom left), and $\geq 4$ jets (bottom right).
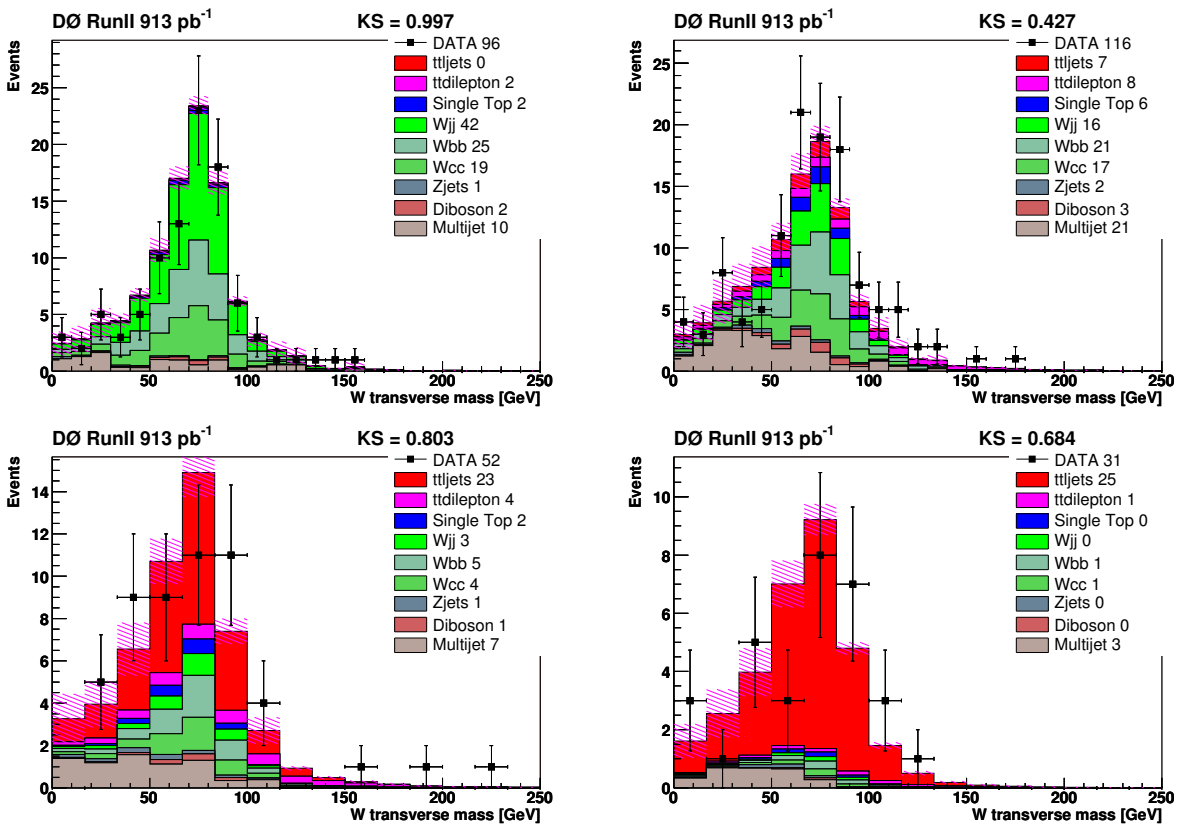

Figure B.21: The $e \nu$ transverse mass distributions for single tagged sample in the $e+$ jets channel. The plots show the data for different jet multiplicities: $=1$ jet (top left), $=2$ jets (top right), $=3$ jets (bottom left), and $\geq 4$ jets (bottom right). 

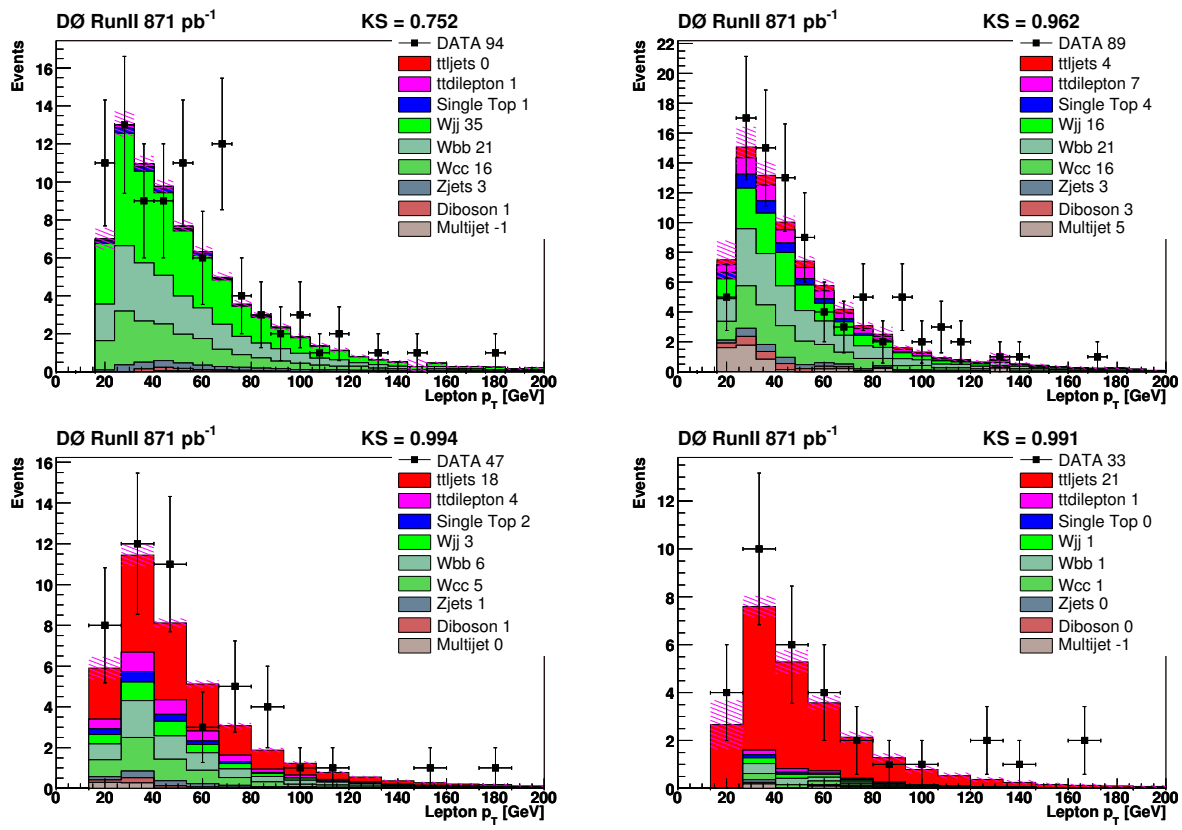

Figure B.22: Muon $p_{\mathrm{T}}$ distributions for single tagged sample in the $\mu+$ jets channel. The plots show the data for different jet multiplicities: $=1$ jet (top left), $=2$ jets (top right), $=3$ jets (bottom left), and $\geq 4$ jets (bottom right).
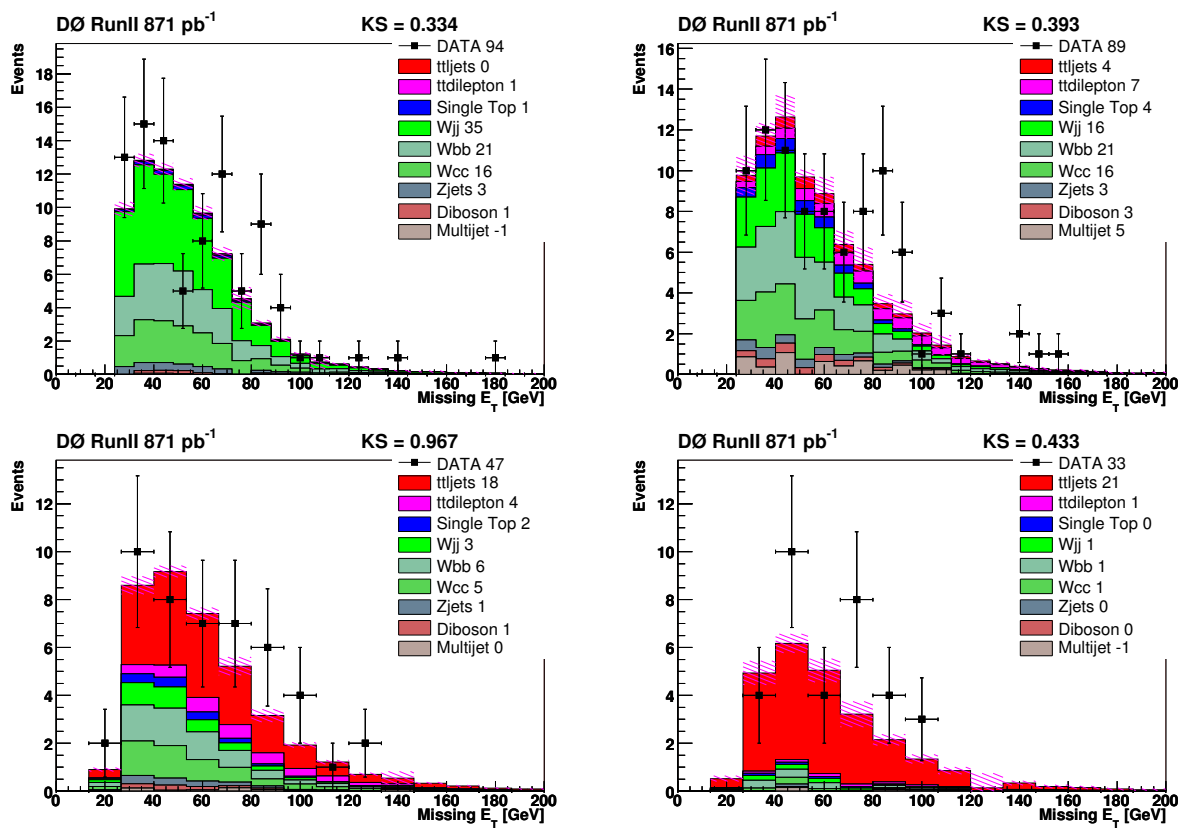

Figure B.23: $E_{T}$ distributions for single tagged sample in the $\mu+$ jets channel. The plots show the data for different jet multiplicities: $=1$ jet (top left), $=2$ jets (top right), $=3$ jets (bottom left), and $\geq 4$ jets (bottom right). 

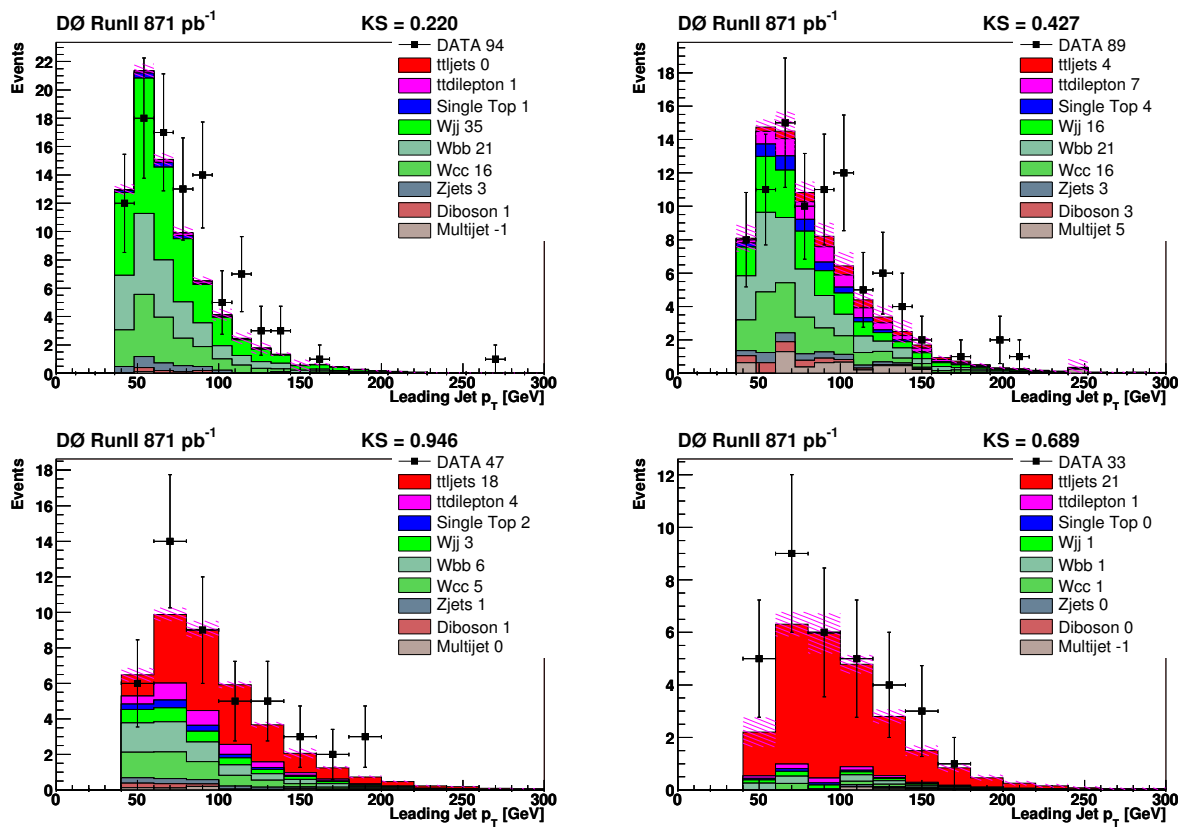

Figure B.24: Leading jet $p_{\mathrm{T}}$ distributions for single tagged sample in the $\mu+$ jets channel. The plots show the data for different jet multiplicities: $=1$ jet (top left), $=2$ jets (top right), $=3$ jets (bottom left), and $\geq 4$ jets (bottom right).
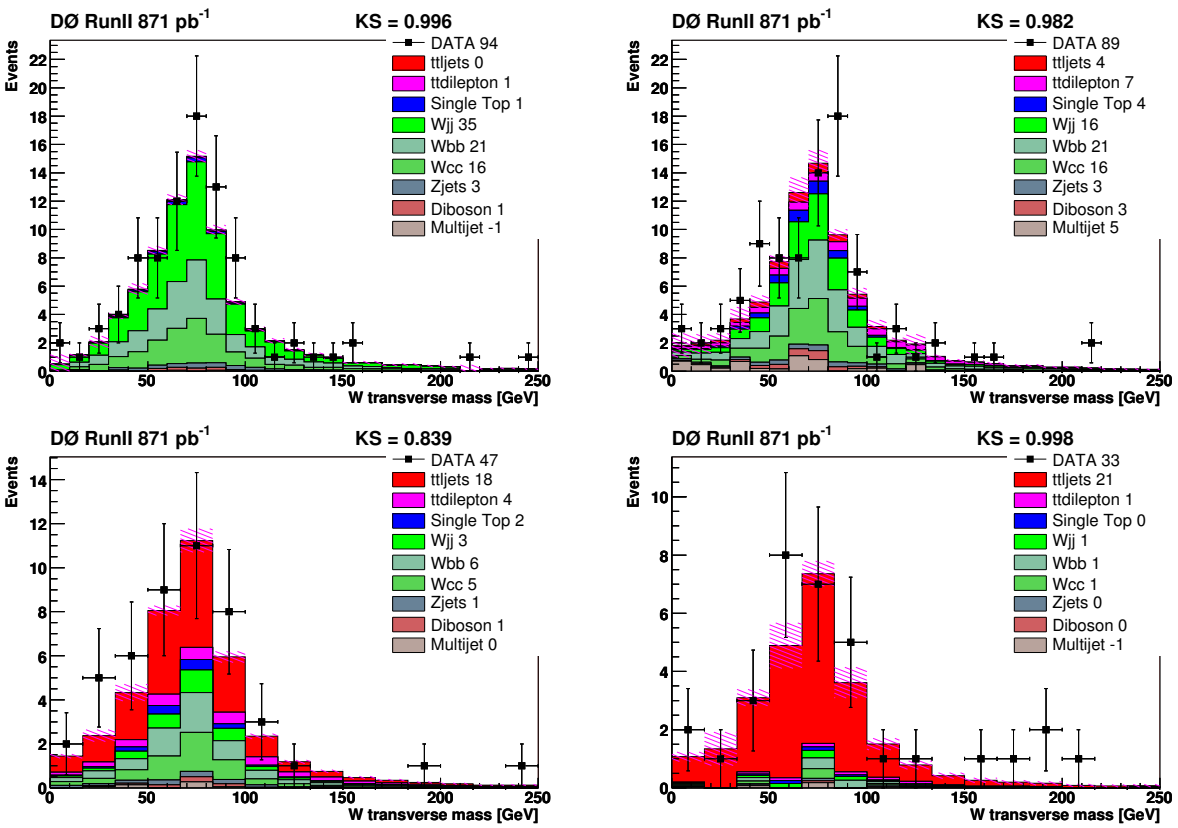

Figure B.25: The $\mu \nu$ transverse mass distributions for single tagged sample in the $\mu+$ jets channel. The plots show the data for different jet multiplicities: $=1$ jet (top left), $=2$ jets (top right), $=3$ jets (bottom left), and $\geq 4$ jets (bottom right). 


\begin{tabular}{|c|c|c|c|c|}
\hline \hline & \multicolumn{4}{|c|}{ e+jets } \\
\hline & 1 jet & 2 jets & 3 jets & $\geq 4$ jets \\
\hline \hline$W j j$ & 42.0 & 15.8 & 2.8 & 0.4 \\
$W b b$ & 24.7 & 21.0 & 5.4 & 0.8 \\
$W c c$ & 19.1 & 16.7 & 4.3 & 0.6 \\
\hline singletop & 1.8 & 5.6 & 1.9 & 0.5 \\
$Z+$ jets & 1.3 & 2.3 & 1.2 & 0.5 \\
Diboson & 1.6 & 3.4 & 0.9 & 0.2 \\
\hline$t \bar{t} \rightarrow l l$ & 1.6 & 8.5 & 4.3 & 0.9 \\
$t \bar{t} \rightarrow$ l+jets & 0.4 & 6.9 & 23.4 & 25.0 \\
\hline Multijet & $10.0 \pm 3.4$ & $21.3 \pm 4.9$ & $7.3 \pm 2.6$ & $2.6 \pm 1.6$ \\
\hline total & $102.4 \pm 4.8$ & $101.5 \pm 6.9$ & $51.6 \pm 3.7$ & $31.6 \pm 2.2$ \\
\hline \hline data & 96.0 & 116.0 & 52.0 & 31.0 \\
\hline \hline difference & $-6.6 \%$ & $+12.5 \%$ & $+0.7 \%$ & $-1.8 \%$ \\
\hline \hline
\end{tabular}

Table B.12: Summary of event yields for the $e+$ jets channel after SLTNN tagging.

\section{B.5.3 Cross Section Calculation}

The cross section calculation discussed in Chapter 6 needs to be modified for this analysis using the SLTNN tagger because the tagged sample is not split into two independent samples. Therefore, the total number of channels are reduced to four: $e+$ jets ( 3 jets and $\geq 4$ jets) and $\mu+$ jets $(3$ jets and $\geq 4$ jets) with the SLTNN b-tagging.

Equation 6.9 and 6.10 can be modified as follows:

$$
\begin{gathered}
N_{W+t \bar{t}}=N_{W+t \bar{t}}^{0 \mathrm{tag}}+N_{W+t \bar{t}}^{\mathrm{tag}} \\
N_{Q C D}=N_{Q C D}^{0 \mathrm{tag}}+N_{Q C D}^{\mathrm{tag}} \\
\mathcal{L}=\mathcal{L}_{0} \times \mathcal{L}_{1} \\
=\mathcal{P}\left(\tilde{N}_{\text {tight }}^{0 \mathrm{tag}} ; N_{\text {tight }}^{0 \mathrm{tag}}\right) \times \mathcal{P}\left(\tilde{N}_{\text {loose-tight }}^{0 \text { tag }} ; N_{\text {loose-tight }}^{\text {tag }}\right) \\
\times \mathcal{P}\left(\tilde{N}_{\text {tight }}^{\mathrm{tag}} ; N_{\text {tight }}^{\mathrm{tag}}\right) \times \mathcal{P}\left(\tilde{N}_{\text {loose-tight }}^{\mathrm{tag}} ; N_{\text {loose-tight }}^{\text {tag }}\right)
\end{gathered}
$$

where "0tag" denotes the sample that is not passed by the SLTNN tagging, and "tag" denotes the 


\begin{tabular}{|c|c|c|c|c|}
\hline \hline & \multicolumn{4}{|c|}{$\mu+$ jets } \\
\hline & 1 jet & 2 jets & 3 jets & $\geq 4$ jets \\
\hline \hline$W j j$ & 34.9 & 15.9 & 3.3 & 0.8 \\
$W b b$ & 21.0 & 20.7 & 6.0 & 1.3 \\
$W c c$ & 15.7 & 15.6 & 5.4 & 1.0 \\
\hline singletop & 1.3 & 4.2 & 1.5 & 0.4 \\
$Z+$ jets & 3.2 & 3.3 & 1.3 & 0.4 \\
Diboson & 1.4 & 3.0 & 0.8 & 0.2 \\
\hline$t \bar{t} \rightarrow$ ll & 1.1 & 6.6 & 3.5 & 0.8 \\
$t \bar{t} \rightarrow$ l+jets & 0.2 & 4.3 & 17.9 & 21.3 \\
\hline Multijet & $-0.7 \pm 1.0$ & $5.0 \pm 2.6$ & $0.5 \pm 0.7$ & $-0.8 \pm 1.0$ \\
\hline total & $78.1 \pm 1.4$ & $78.6 \pm 3.7$ & $40.2 \pm 1.0$ & $25.5 \pm 1.5$ \\
\hline \hline data & 94.0 & 89.0 & 47.0 & 33.0 \\
\hline \hline difference & $+16.9 \%$ & $+11.7 \%$ & $+14.4 \%$ & $+22.7 \%$ \\
\hline \hline
\end{tabular}

Table B.13: Summary of event yields for the $\mu+$ jets channel after SLTNN tagging.

sample that is passed by the SLTNN tagging. The solution, Equation 6.18 and 6.19 , can be modified as follows:

$$
\begin{aligned}
N_{\text {tight }}^{\mathrm{tag}}= & \frac{1}{1-(a-c)+(b-c) P_{W}^{t a g}} \times \\
& \left(k+P_{W}^{\text {tag }}(b-c) N_{\text {tight }}^{0 t a g}+c\left(1-P_{W}^{\text {tag }}\right) N_{\text {loose-tight }}^{\text {tag }}-c P_{W}^{\text {tag }} N_{\text {loose-tight }}^{\text {Otag }}\right) \\
k= & N_{t \bar{t}}^{\text {tag }}-P_{W}^{t a g} N_{M C b k g}-P_{W}^{t a g} N_{t \bar{t}}+N_{M C b k g}^{\text {tag }} \\
a= & \frac{\varepsilon_{Q C D}}{\varepsilon_{s i g}-\varepsilon_{Q C D}} \\
b= & \frac{\varepsilon_{\text {sig }}}{\varepsilon_{\text {sig }}-\varepsilon_{Q C D}} \\
c= & \frac{\varepsilon_{s i g} \varepsilon_{Q C D}}{\varepsilon_{s i g}-\varepsilon_{Q C D}} .
\end{aligned}
$$

The solution $N_{\text {tight }}^{\mathrm{tag}}$ is inserted into the likelihood (Equation B.14). The observed number of events $\left(N_{\text {tight }}^{0 \text { tag }}, N_{\text {loose-tight }}^{\text {tag }}\right.$ and $\left.N_{\text {loose-tight }}^{\text {otag }}\right)$, are inserted into the same likelihood function. The input numbers are floated in the fit to find the optimized values using the same procedure discussed in Chapter 6.

For the optimized operating point (loose muon, loose NN), the result for $t \bar{t}$ pair production cross 
section using the SLTNN tagging is

$$
\begin{array}{ccc}
\mathrm{e}+\text { jets }: & \sigma_{p \bar{p} \rightarrow t \bar{t}+X}=6.75_{-1.26}^{+1.36} \text { (stat) } \mathrm{pb} \\
\mu+\text { jets }: & \sigma_{p \bar{p} \rightarrow t \bar{t}+X}=9.30_{-1.55}^{+1.68} \text { (stat) } \mathrm{pb} \\
\ell+\text { jets }: & \sigma_{p \bar{p} \rightarrow t \bar{t}+X}=7.88_{-1.00}^{+1.05} \text { (stat) } \mathrm{pb} .
\end{array}
$$

Figure B.26 shows the signal and background compositions estimated using MC samples and are compared to the number of events in data sample as a function of jet multiplicity. The measured cross section for all channels $(7.88 \mathrm{pb})$ is used to create this plot.

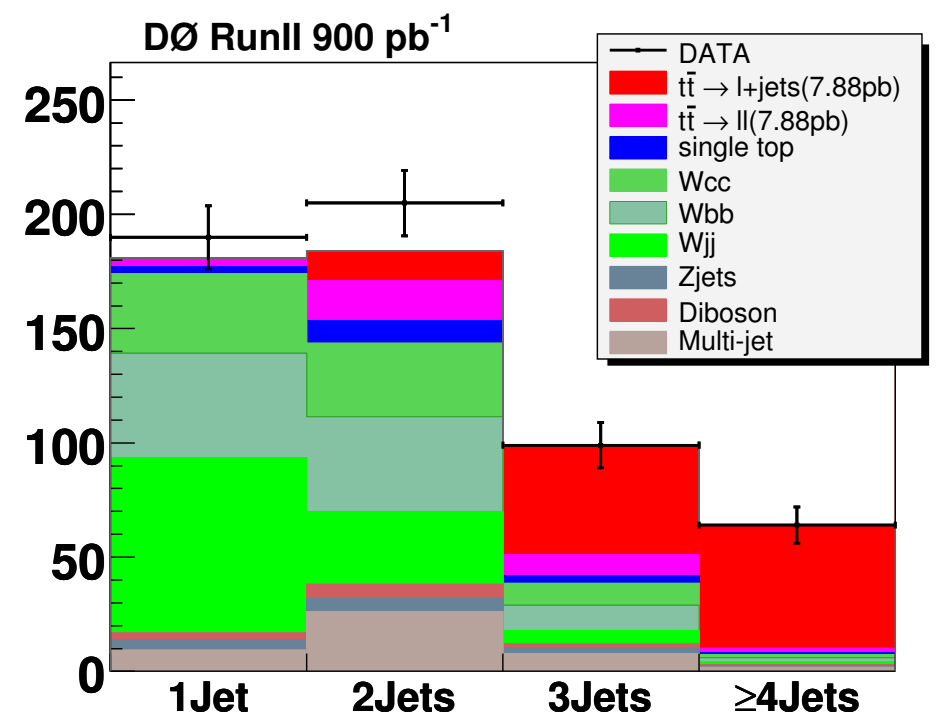

Figure B.26: The signal and background composition predicted using MC samples and the number of events in the data sample as a function of jet multiplicity for the SLTNN tagging algorithm. The plot shows the result in the lepton+jets channel.

\section{B.5.4 Systematic Uncertainty}

To estimate the systematic uncertainties for this analysis, the same list of systematic sources (shown in Section 6.3) is used. Table B.14 shows the systematic uncertainties estimated for each source. 


\begin{tabular}{|c|c|c|c|c|c|c|c|}
\hline & & \multicolumn{2}{|c|}{$e+j e t s$} & \multicolumn{2}{|c|}{$\mu+$ jets } & \multicolumn{2}{|c|}{$l+j e t s$} \\
\hline type & source & $\overline{\sigma^{+}}$ & $\overline{\sigma^{-}}$ & $\overline{\sigma^{+}}$ & $\sigma^{-}$ & $\overline{\sigma^{+}}$ & $\overline{\sigma^{-}}$ \\
\hline \multirow{3}{*}{ preselection } & Luminosity Reweighting & +0.06 & -0.00 & +0.08 & -0.00 & +0.07 & -0.00 \\
\hline & Primary Vertex & +0.11 & -0.10 & +0.15 & -0.14 & +0.12 & -0.12 \\
\hline & $Z p_{\mathrm{T}}$ Reweighting & +0.00 & -0.00 & +0.00 & -0.03 & +0.00 & -0.01 \\
\hline \multirow{7}{*}{$e+j e t s$ only } & $E M I D$ & +0.18 & -0.17 & - & - & +0.12 & -0.12 \\
\hline & L1EMtrigger & +0.07 & -0.01 & - & - & +0.05 & -0.01 \\
\hline & L2EMtrigger & +0.00 & -0.00 & - & - & +0.00 & -0.00 \\
\hline & L3EMtrigger & +0.04 & -0.04 & - & - & +0.03 & -0.03 \\
\hline & L1JetTrigger & +0.00 & -0.00 & - & - & +0.00 & -0.00 \\
\hline & L2JetTrigger & +0.00 & -0.00 & - & - & +0.00 & -0.00 \\
\hline & L3JetTrigger & +0.00 & -0.00 & - & - & +0.00 & -0.00 \\
\hline \multirow{4}{*}{$\mu+$ jets only } & Muon ID & - & - & +0.07 & -0.07 & +0.02 & -0.02 \\
\hline & Muon Tracking & - & - & +0.07 & -0.07 & +0.02 & -0.02 \\
\hline & Muon Isolation & - & - & +0.19 & -0.19 & +0.07 & -0.07 \\
\hline & Muon Trigger & - & - & +0.26 & -0.23 & +0.09 & -0.08 \\
\hline \multirow{4}{*}{ Jets } & Jet Trigger & +0.01 & -0.01 & +0.02 & -0.02 & +0.02 & -0.02 \\
\hline & $J E S$ & +0.36 & -0.29 & +0.43 & -0.34 & +0.39 & -0.31 \\
\hline & $J S S R$ & +0.00 & -0.09 & +0.00 & -0.15 & +0.00 & -0.12 \\
\hline & Jet Identification & +0.11 & -0.11 & +0.06 & -0.06 & +0.09 & -0.09 \\
\hline \multirow{2}{*}{ Matrix Method } & $\varepsilon_{s i g}$ & +0.03 & -0.03 & +0.08 & -0.08 & +0.05 & -0.05 \\
\hline & $\varepsilon_{Q C D}$ & +0.06 & -0.06 & +0.08 & -0.07 & +0.02 & -0.02 \\
\hline \multirow{3}{*}{ MC Modeling } & W HF Scale Factor & +0.14 & -0.13 & +0.20 & -0.19 & +0.17 & -0.16 \\
\hline & b-fragmetation & +0.18 & -0.00 & +0.18 & -0.00 & +0.19 & -0.00 \\
\hline & Factorization & +0.03 & -0.02 & +0.04 & -0.03 & +0.03 & -0.02 \\
\hline \multirow{4}{*}{ b-tagging } & $F D T$ & +0.00 & -0.09 & +0.00 & -0.12 & +0.00 & -0.11 \\
\hline & b-jets TRF & +0.55 & -0.44 & +0.75 & -0.56 & +0.64 & -0.49 \\
\hline & $c$-jets TRF & +0.16 & -0.09 & +0.23 & -0.11 & +0.19 & -0.10 \\
\hline & light-jets TRF & +0.03 & -0.03 & +0.04 & -0.04 & +0.03 & -0.03 \\
\hline \multirow{6}{*}{ Others } & Z HF Scale Factor & +0.06 & -0.06 & +0.08 & -0.08 & +0.07 & -0.07 \\
\hline & Signal Modeling & +0.00 & -0.21 & +0.00 & -0.25 & +0.00 & -0.23 \\
\hline & $M C$ cross section & +0.03 & -0.03 & +0.03 & -0.03 & +0.03 & -0.03 \\
\hline & $M C$ statistics on signal & +0.01 & -0.01 & +0.01 & -0.01 & +0.01 & -0.01 \\
\hline & MC statistics on background & +0.04 & -0.03 & +0.05 & -0.04 & +0.04 & -0.03 \\
\hline & $P D F$ & +0.02 & -0.02 & +0.02 & -0.02 & +0.02 & -0.02 \\
\hline \multicolumn{2}{|r|}{ Total } & +0.77 & -0.66 & +1.03 & -0.84 & +0.86 & -0.72 \\
\hline
\end{tabular}

Table B.14: Summary of total systematic unertainties for combined results (all channels). 


\section{B.5.5 Conclusion}

The final result for all channels is

$$
\ell+\text { jets }: \quad \sigma_{p \bar{p} \rightarrow t \bar{t}+X}=7.88_{-1.00}^{+1.05} \text { (stat) }{ }_{-0.72}^{+0.86} \text { (sys) } \pm 0.48 \text { (lumi) pb. }
$$

Figure B.27 shows the various results for $t \bar{t}$ production cross section in both CDF and DØ collaborations to compare with the result using the SLTNN algorithm (5th from top). The red bars denote the statistical uncertainty and the blue bars denote the total uncertainty for the measurement.

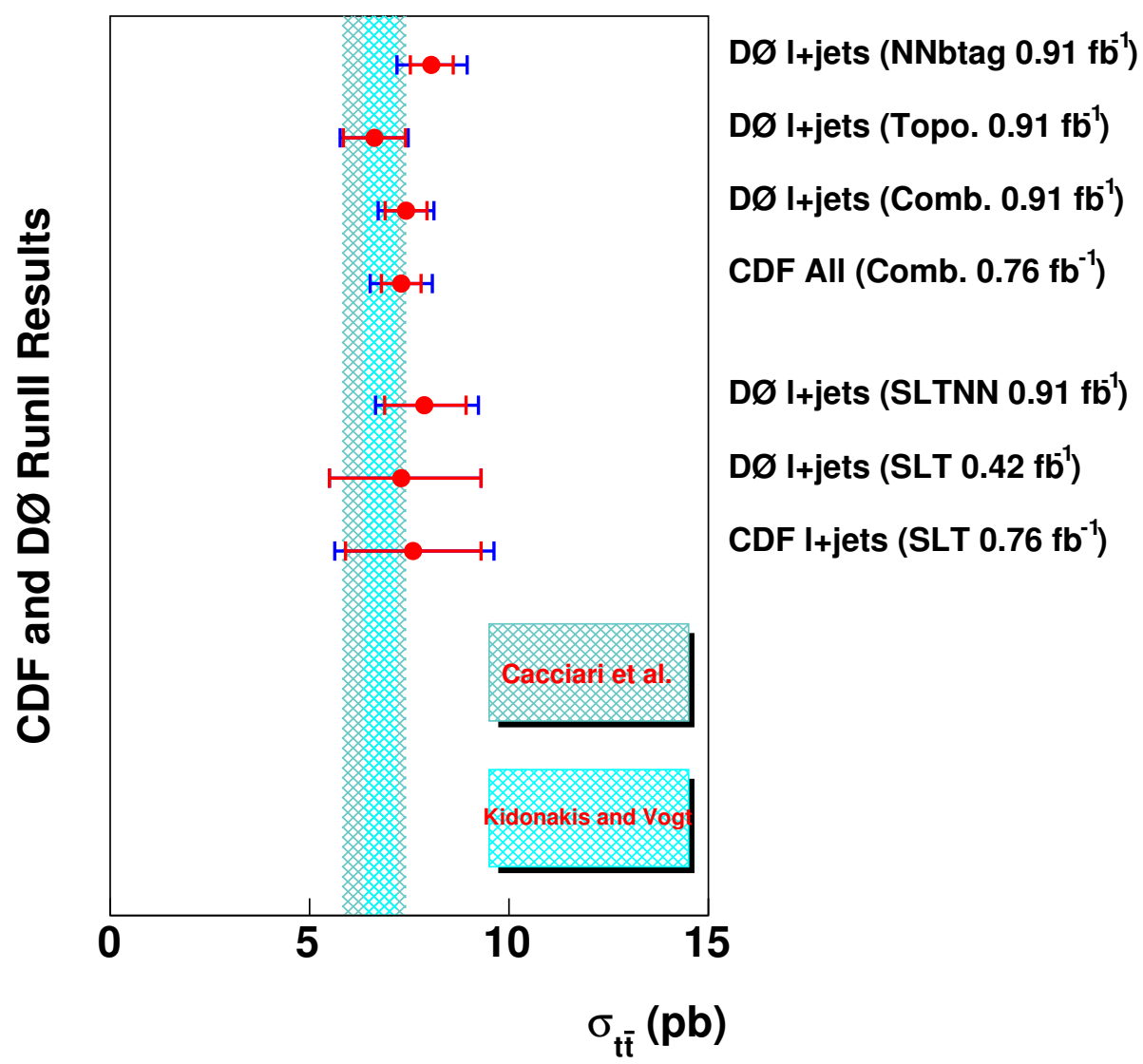

Figure B.27: Summary of the various $t \bar{t}$ cross section measurements made by CDF and $\mathrm{D} \varnothing$. The shade areas denote the theoretical cross sections with its uncertainty. The red bars denote the statistical uncertainty and the blue bars denote the total uncertainty for the measurement. 


\section{Appendix $\mathrm{C}$}

\section{Control Plots}

In this chapter, control plots for the kinematic variables, Centrality, Sphericity, $H_{T}, \Delta \phi$ (leading jet, $\left.E_{T}\right)$, and $\Delta \phi\left(\right.$ lepton, $\left.E_{T}\right)$ are shown for each jet multiplicity bin. The defintions of the kinematic variables are as below. Three samples (preselection, exactly one b-tagged, and two or more b-tagged samples) are presented individually.

- $H_{T}$ : sum of the transverse momentum of all jets $\left(\sum E_{T}\right)$ with $E_{t}>15$ and $|\eta|<2.5$

- Centrality : $H_{T} / H$, where $H$ is the scalar sum of the jet energies

- Sphericity : $S=\frac{3}{2}\left(\lambda_{2}+\lambda_{3}\right)$, where $\lambda_{2}$ and $\lambda_{3}$ are the smallest eigenvalues of the normalized momentum tensor $\mathrm{M}$

- $\Delta \phi\left(\right.$ leading jet, $\left.\mathbb{E}_{T}\right): \Delta \phi$ between the leading jet and $\mathbb{E}_{T}$

- $\Delta \phi\left(\right.$ lepton, $\left.\mathbb{E}_{T}\right): \Delta \phi$ between the lepton and $\mathbb{E}_{T}$.

The figures show these distributions for the four jet multiplicity bins: $=1$ jet (top left), $=2$ jets (top right), $=3$ jets (bottom left), and $\geq 4$ jets (bottom right). 


\section{C.1 Control Plots for the $e+$ jets Channel}

\section{C.1.1 Preselection Events}
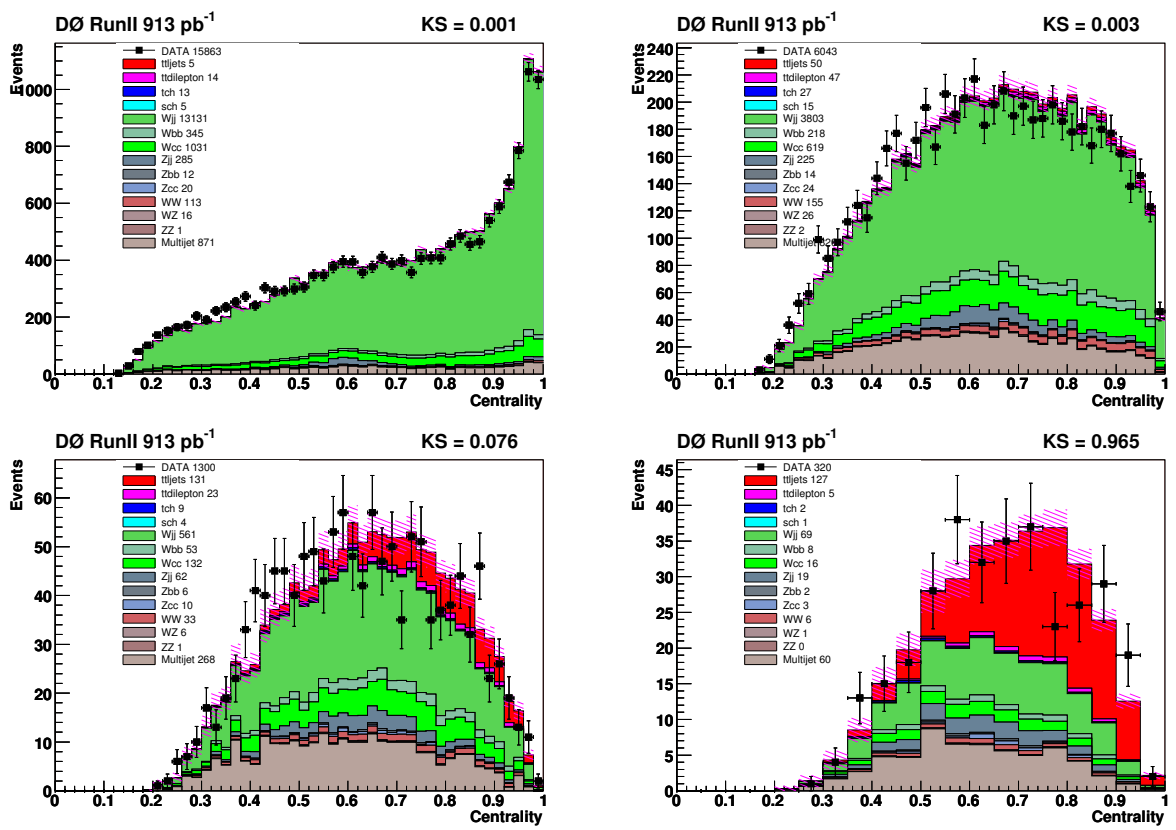

Figure C.1: Centrality distributions for the preselected sample in the $e+$ jets channel. 

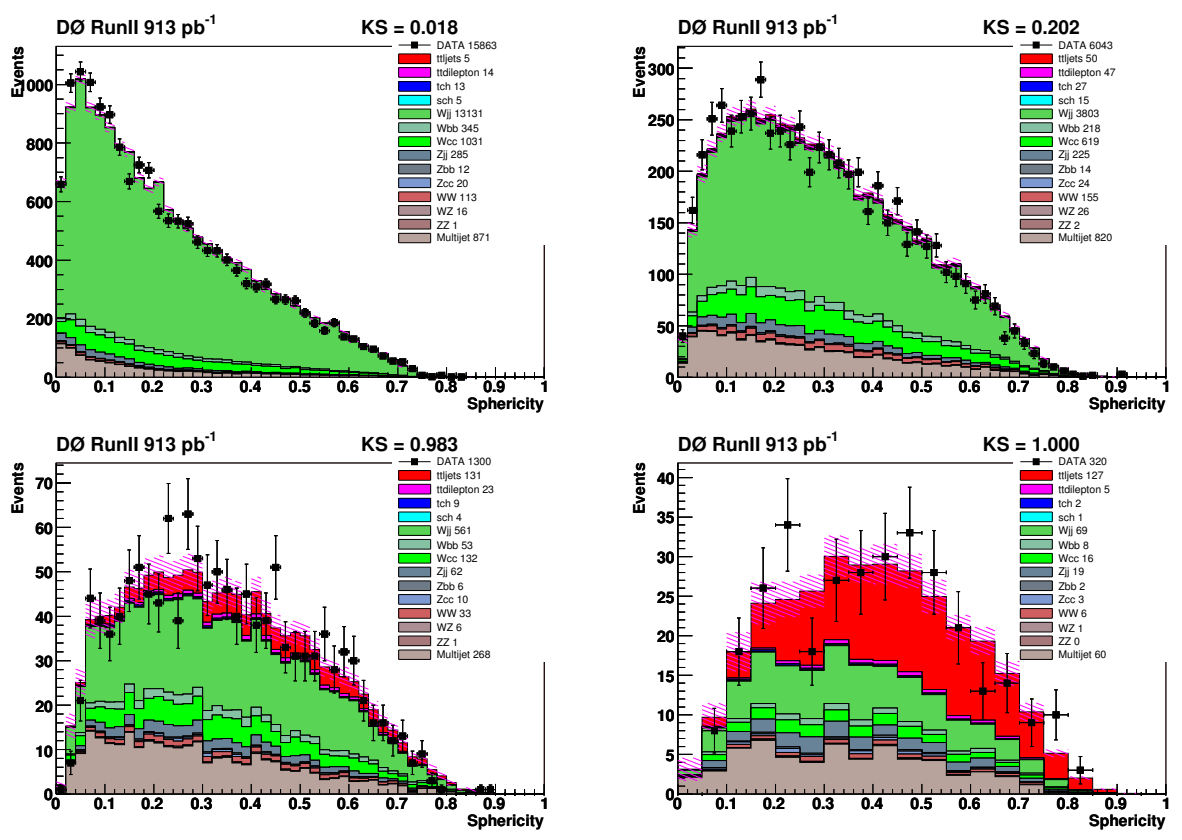

Figure C.2: Sphericity distributions for the preselected sample in the $e+$ jets channel.
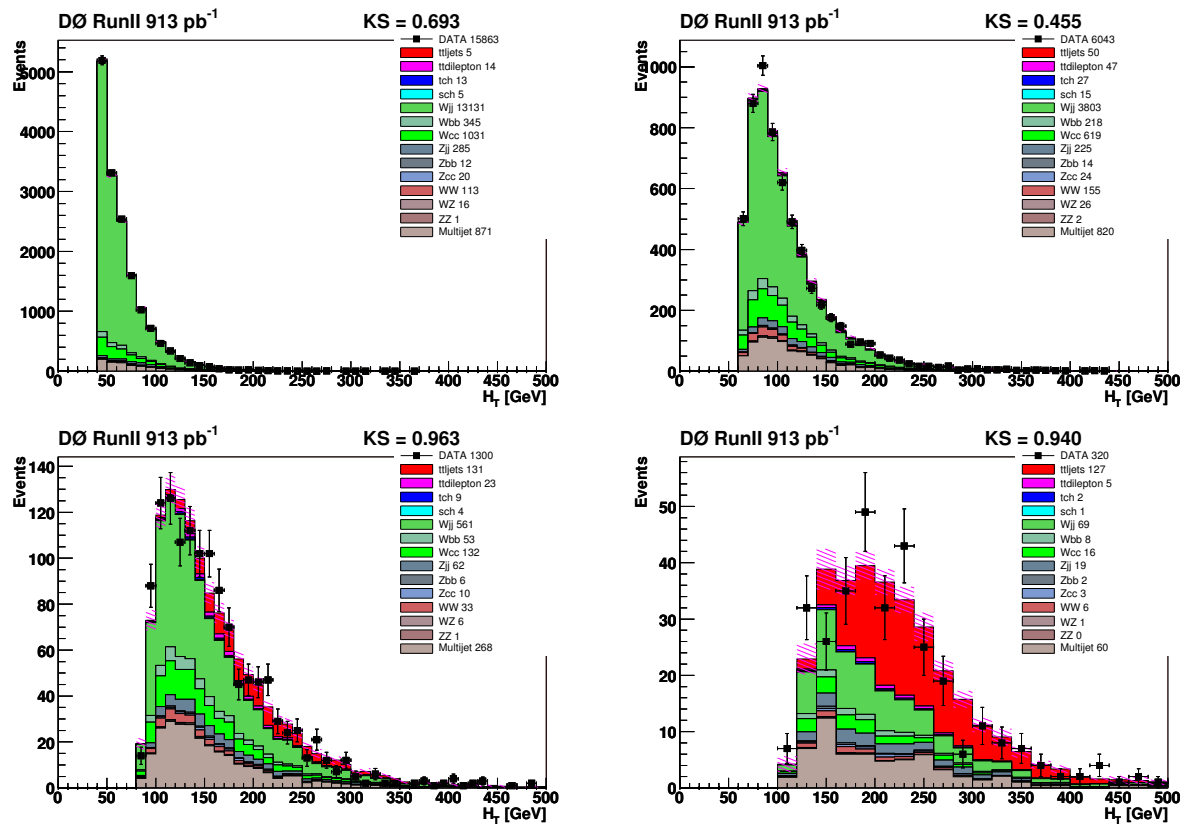

Figure C.3: $H_{T}$ distributions for the preselected sample in the $e+$ jets channel. 

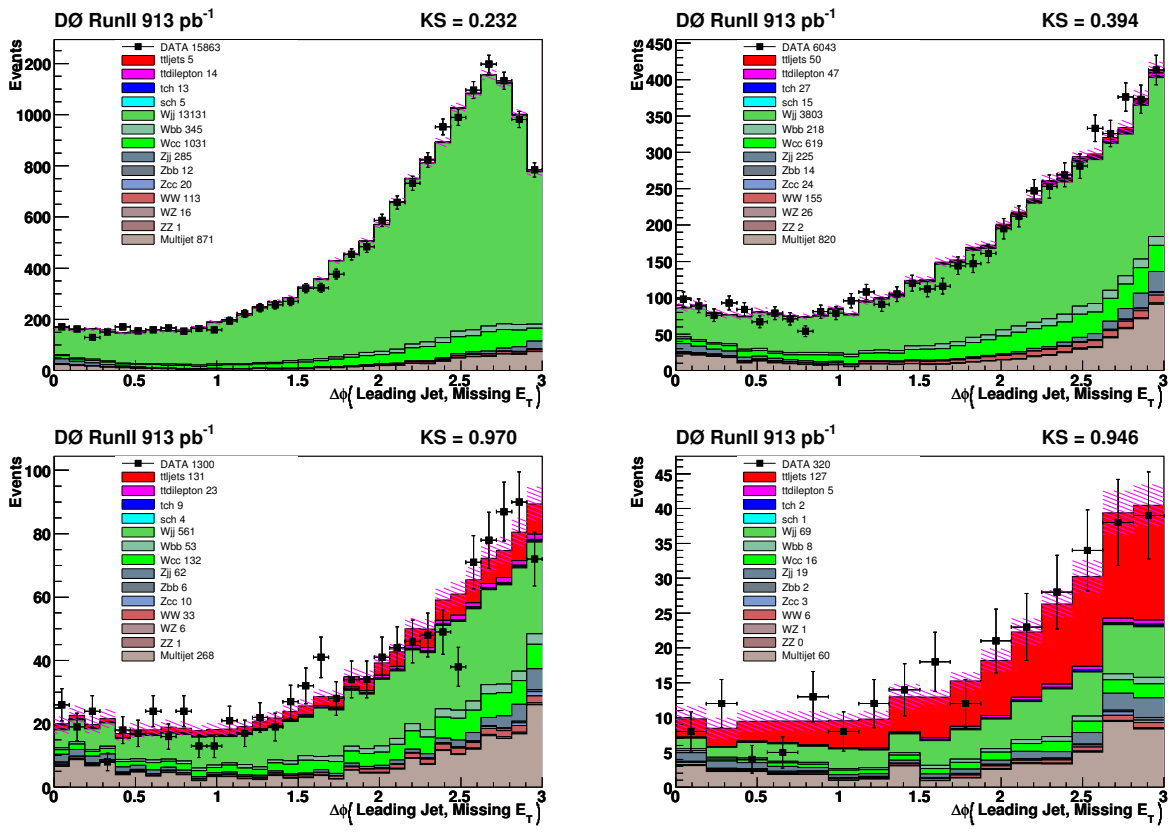

Figure C.4: $\Delta \phi$ (leading Jet, $\mathbb{E}_{T}$ ) distributions for the preselected sample in the $e+$ jets channel.
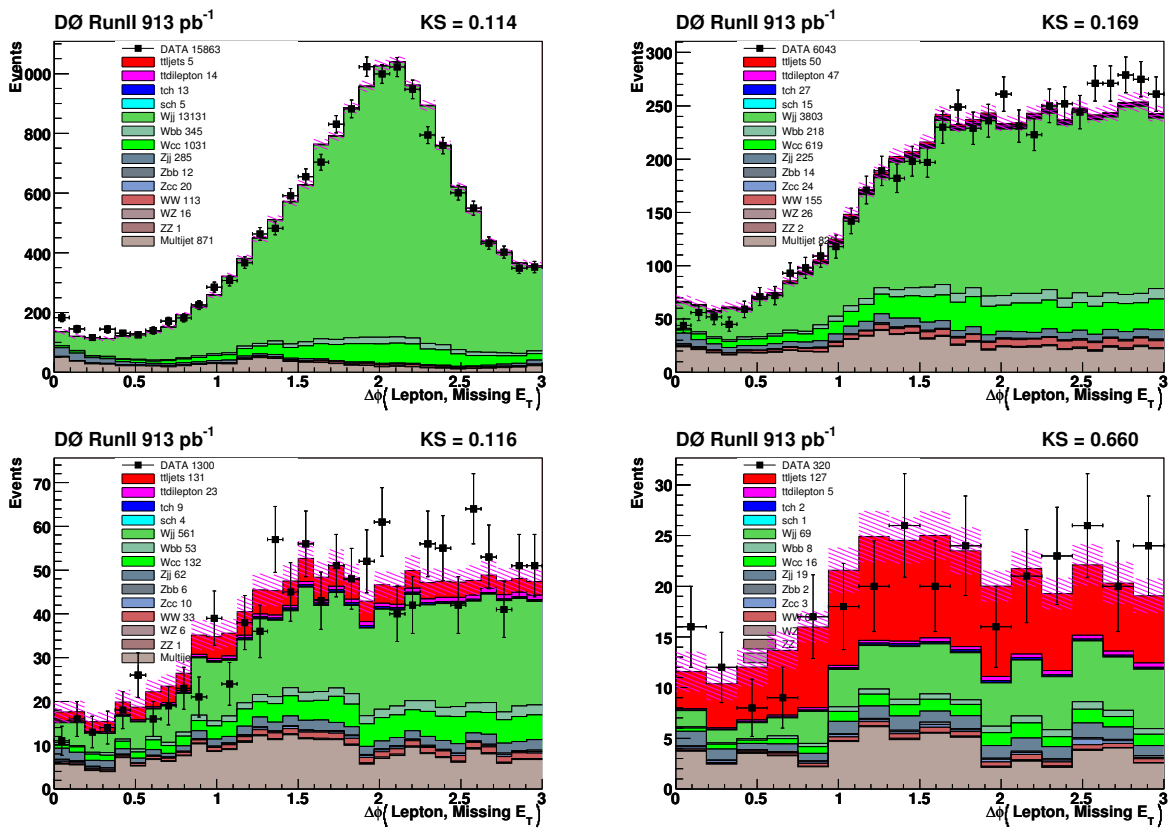

Figure C.5: $\Delta \phi\left(\right.$ electron,$\left.E_{T}\right)$ distributions for the preselected sample in the $e+$ jets channel. 


\section{C.1.2 Exactly One b-tagged Events}
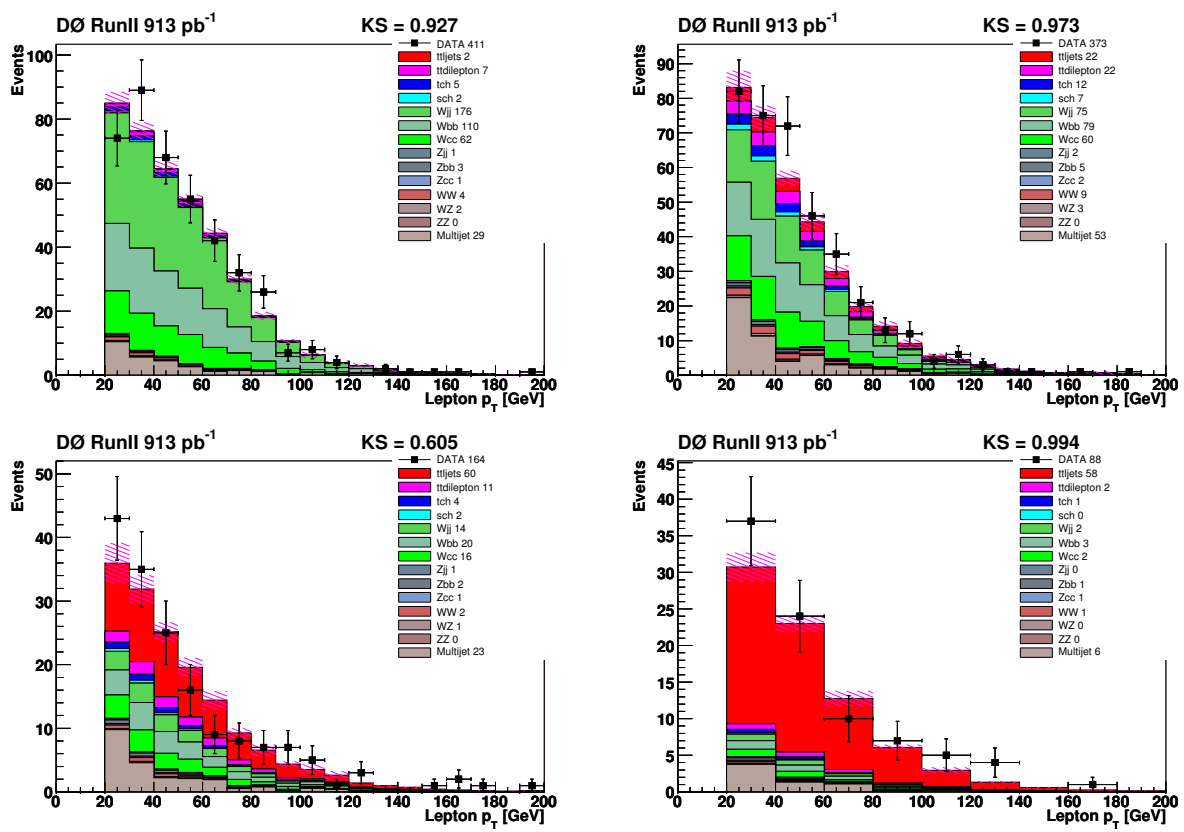

Figure C.6: Electron $p_{\mathrm{T}}$ distributions for the exactly one tagged sample in the e+jets channel. 

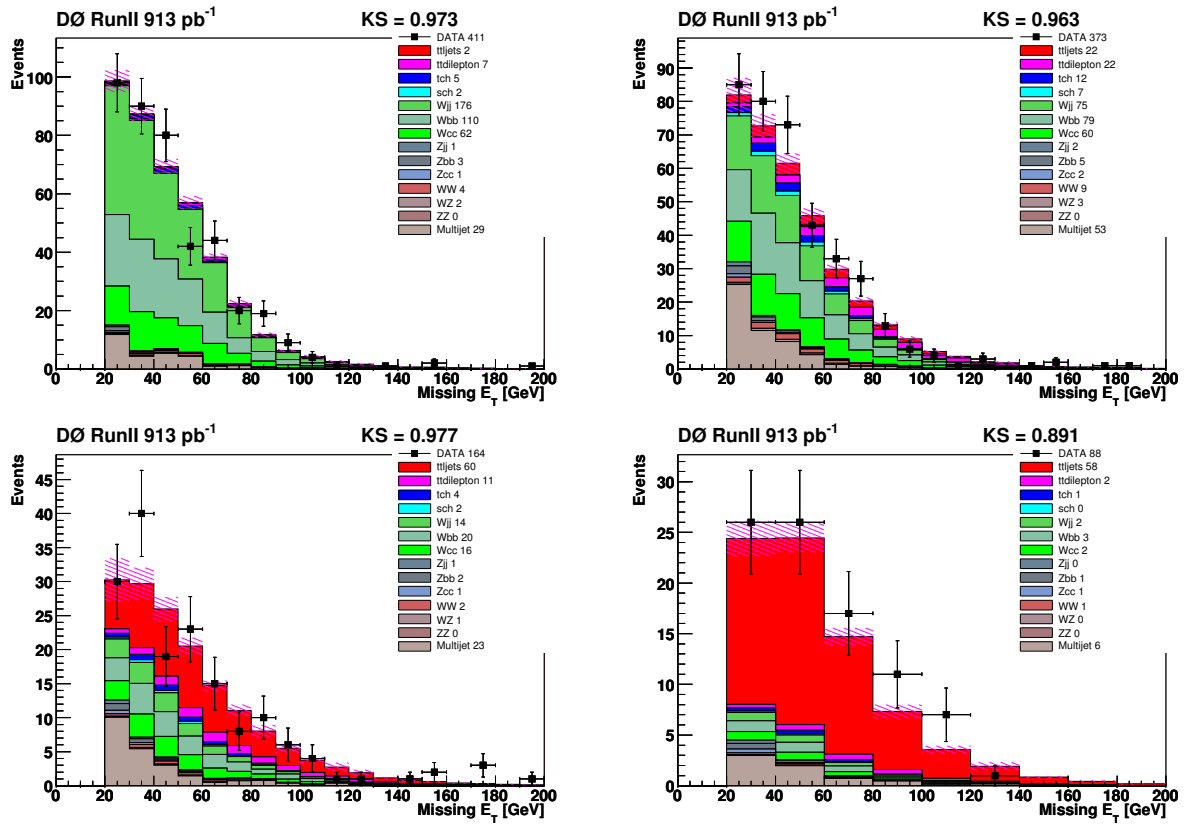

Figure C.7: $E_{T}$ distributions for the exactly one tagged sample in the $e+$ jets channel.
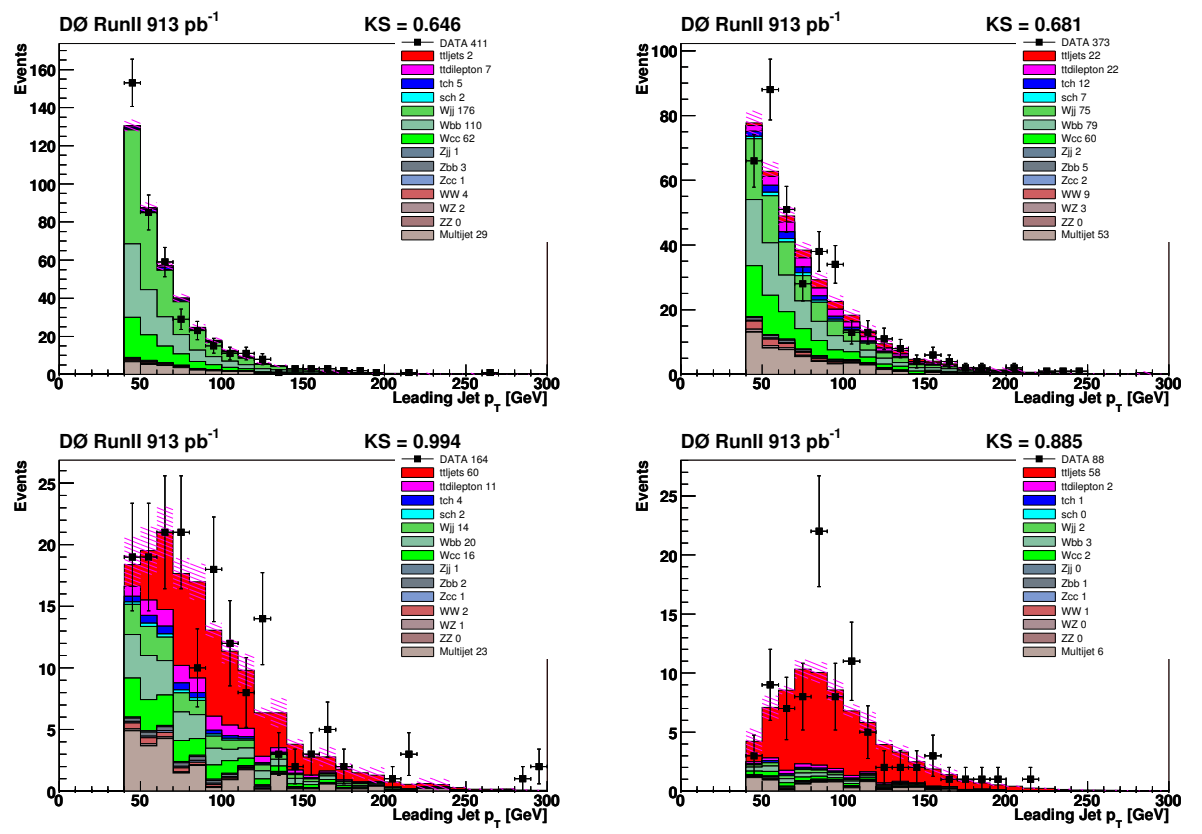

Figure C.8: Leading jet $p_{\mathrm{T}}$ distributions for the exactly one tagged sample in the $e+$ jets channel. 

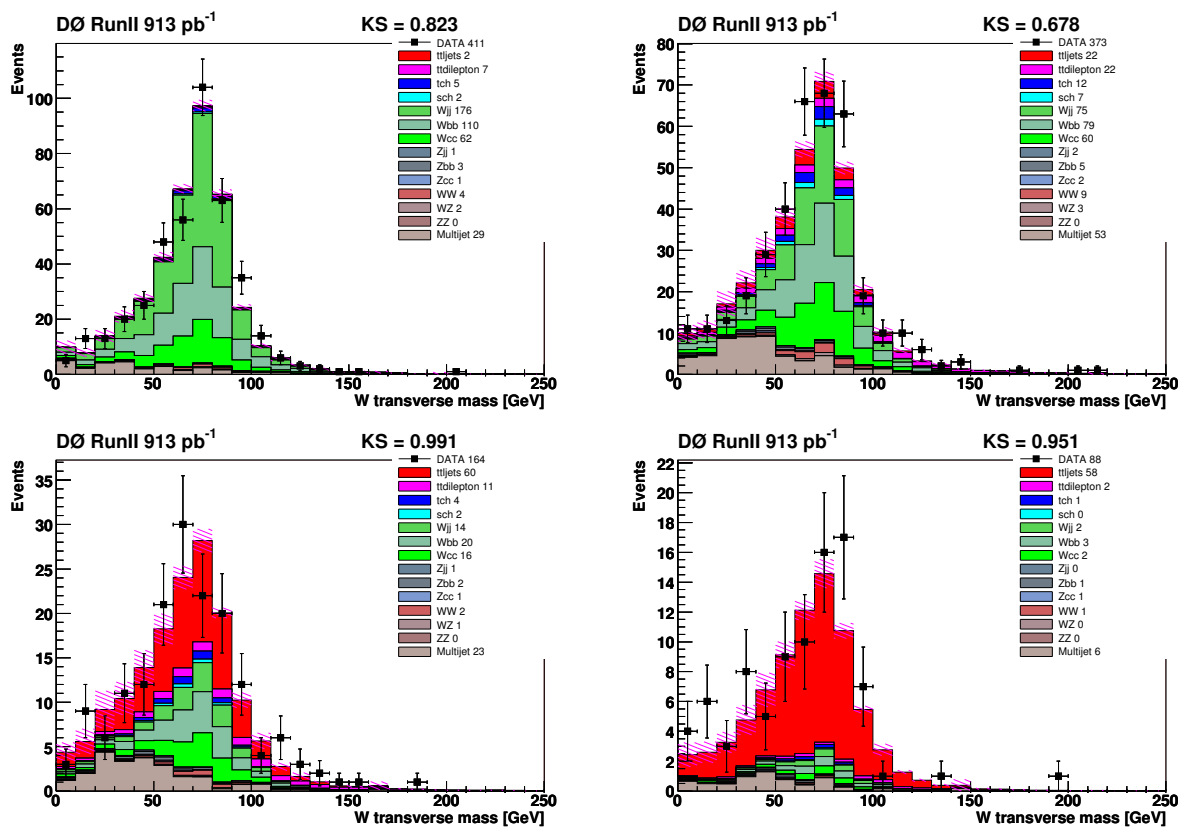

Figure C.9: The $e \nu$ transverse mass distributions for the exactly one tagged sample in the $e+$ jets channel.
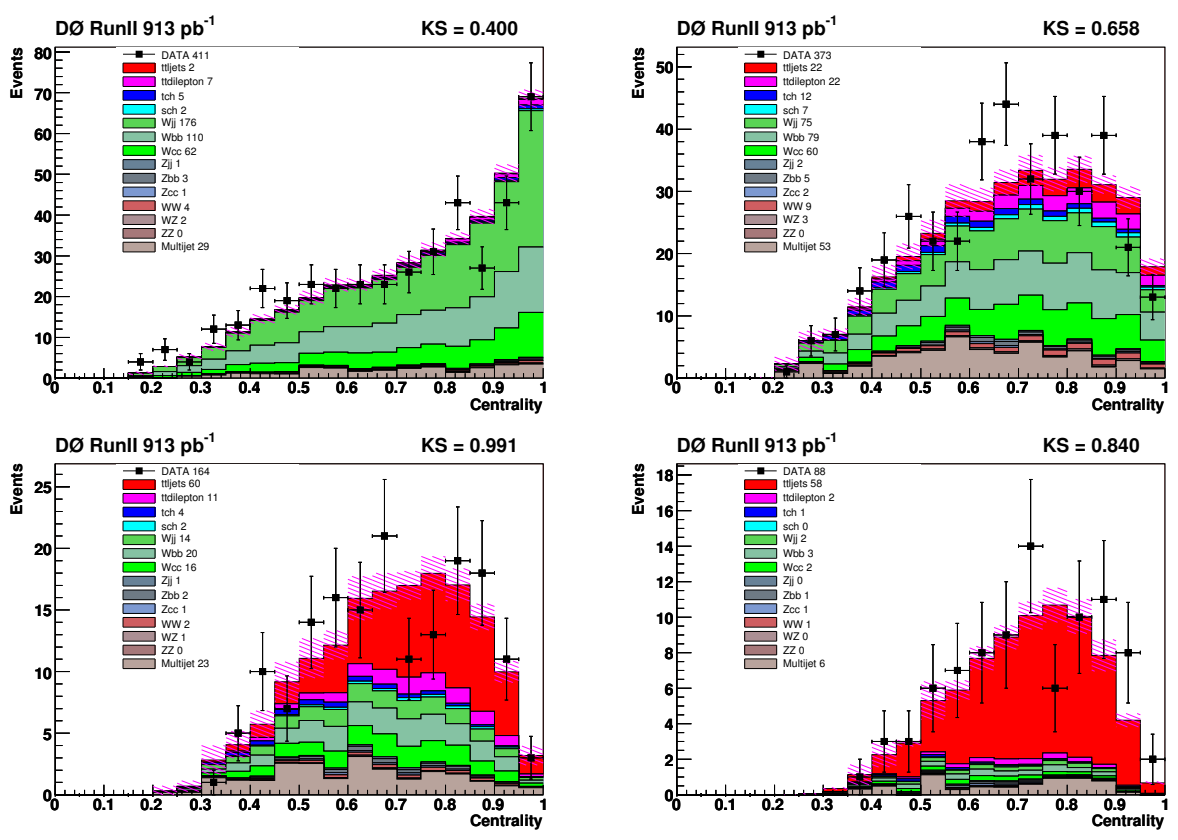

Figure C.10: Centrality distributions for the exactly one tagged sample in the $e+$ jets channel. 

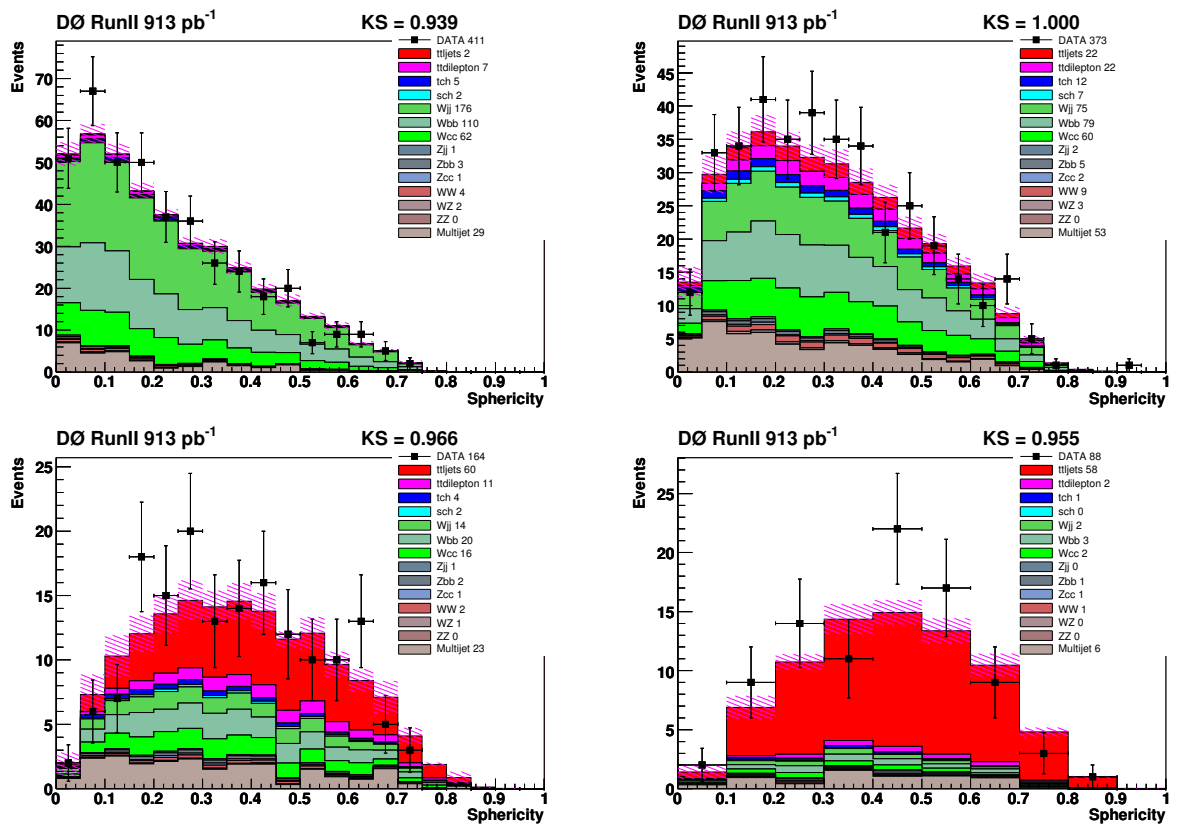

Figure C.11: Sphericity distributions for the exactly one tagged sample in the $e+$ jets channel.
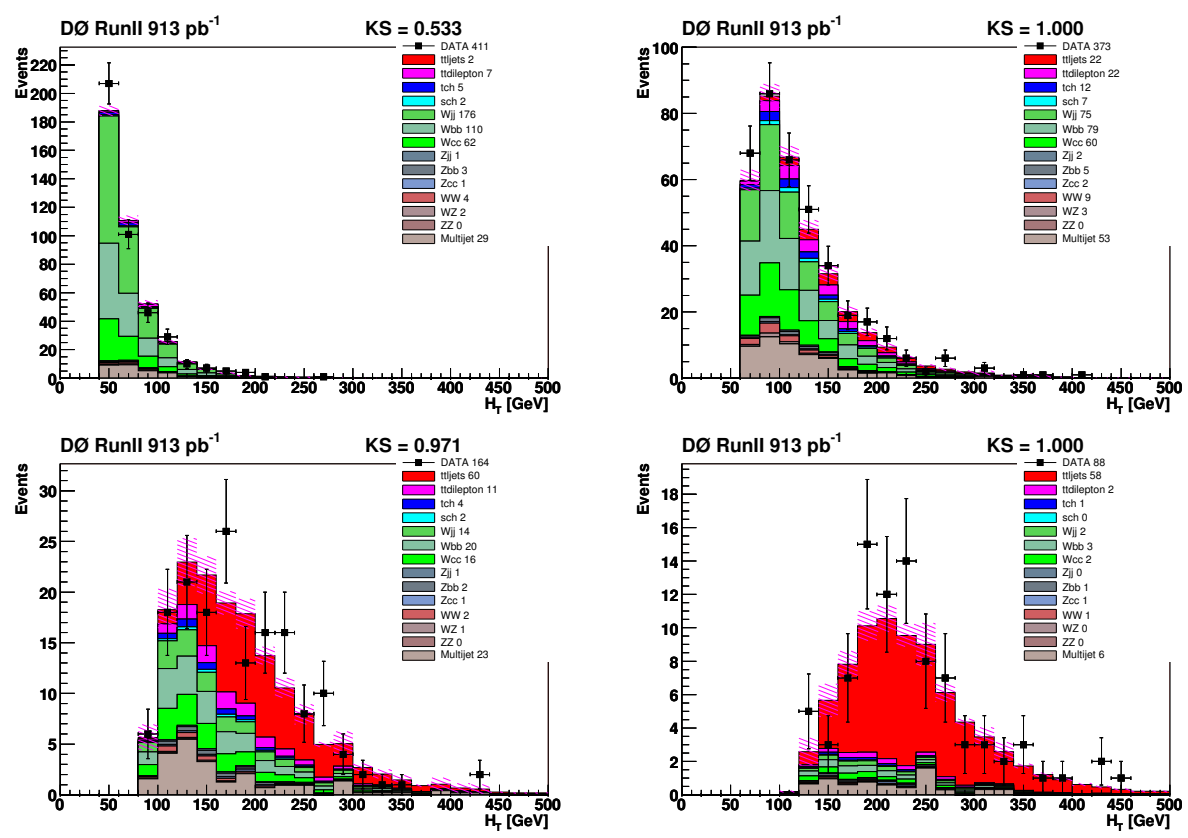

Figure C.12: $H_{T}$ distributions for the exactly one tagged sample in the $e+$ jets channel. 

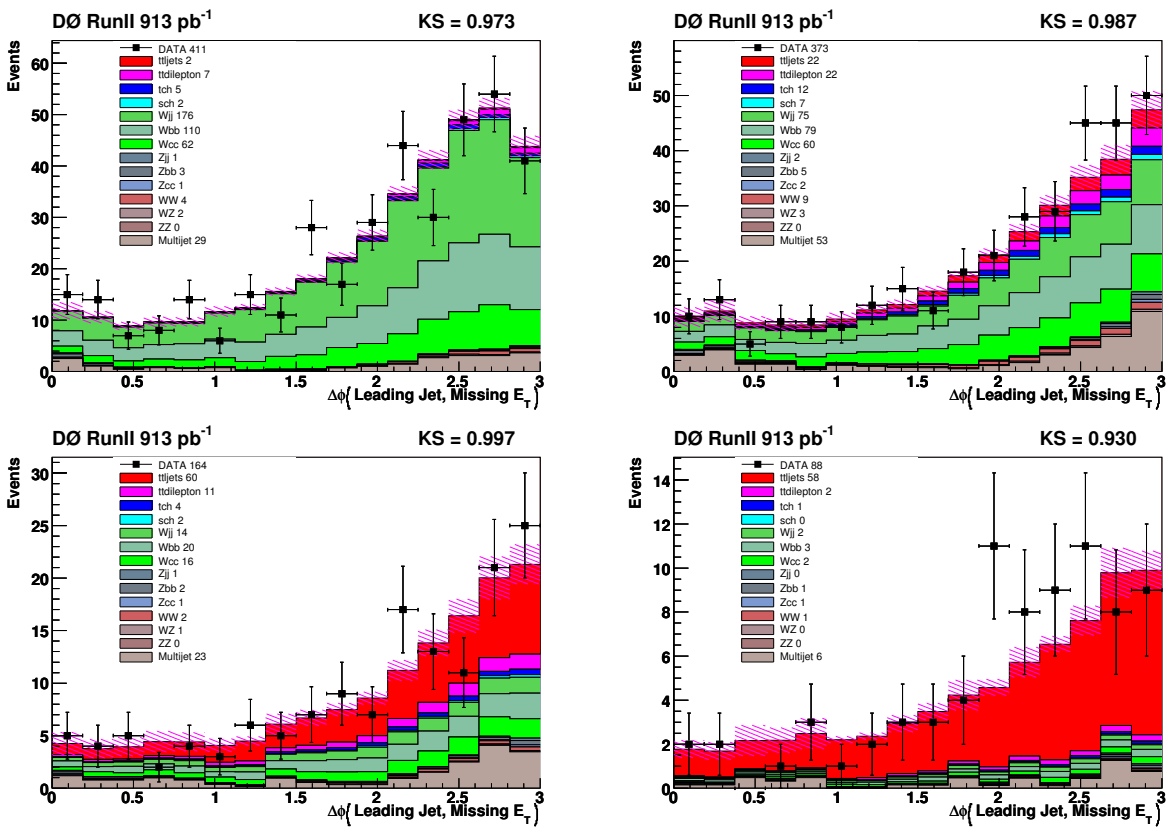

Figure C.13: $\Delta \phi$ (leading Jet, $\mathbb{E}_{T}$ ) distributions for the exactly one tagged sample in the $e+$ jets channel.
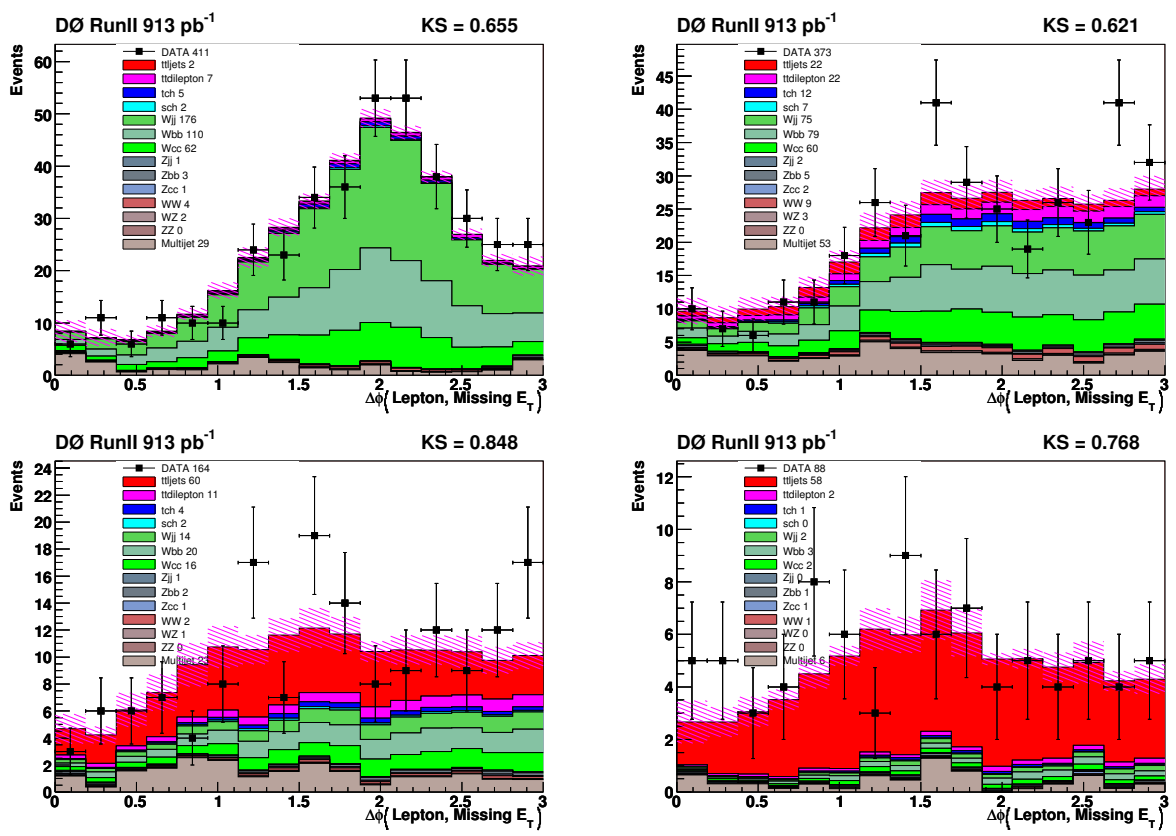

Figure C.14: $\Delta \phi\left(\right.$ electron, $\left.E_{T}\right)$ distributions for the exactly one tagged sample in the $e+$ jets channel. 


\section{C.1.3 Two or More b-tagged Events}

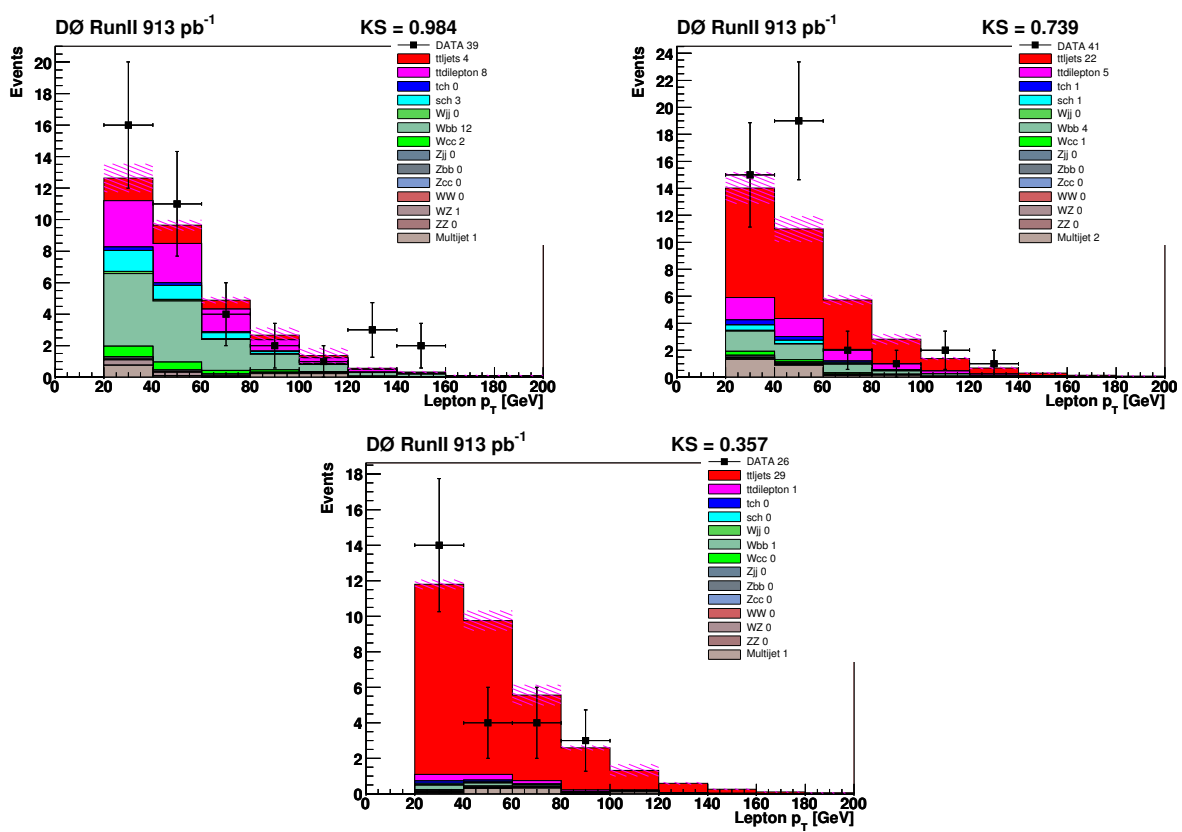

Figure C.15: Electron $p_{\mathrm{T}}$ distributions for the two or more tagged sample in the $e+$ jets channel. 

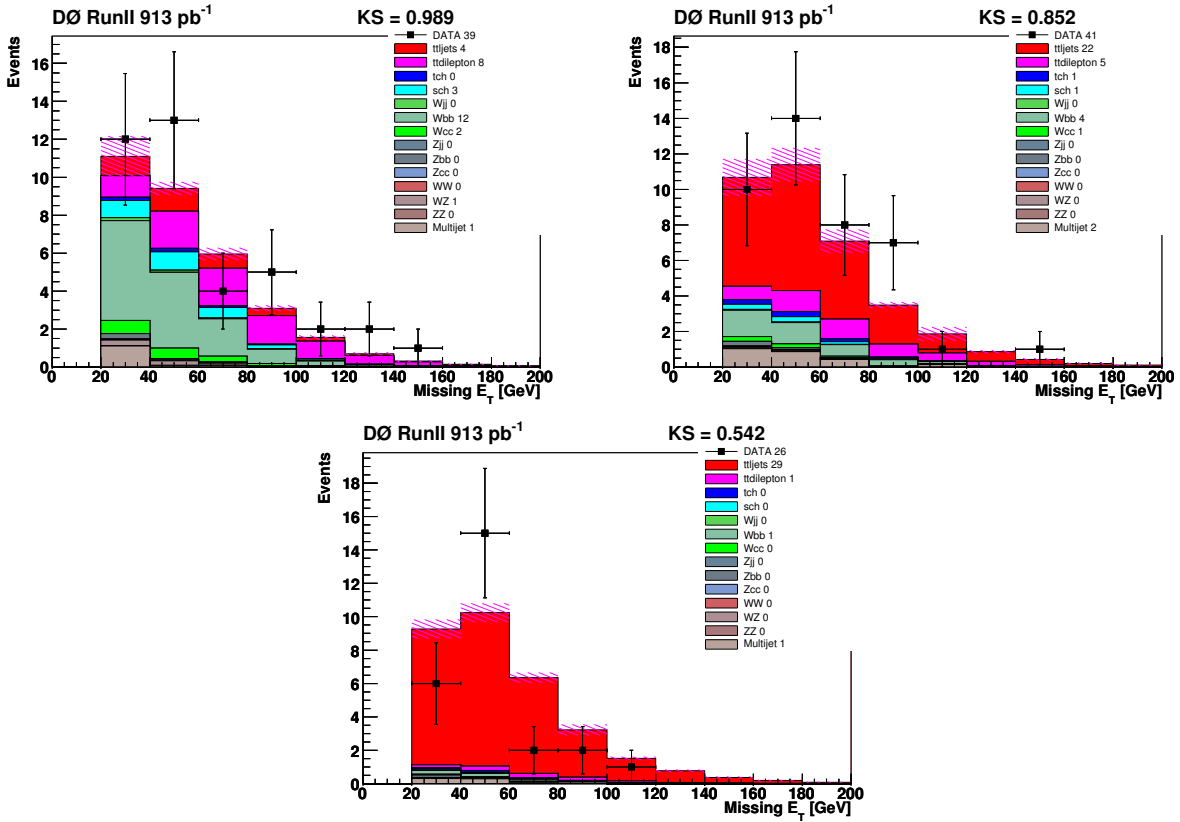

Figure C.16: $\not_{T}$ distributions for the two or more tagged sample in the $e+$ jets channel.
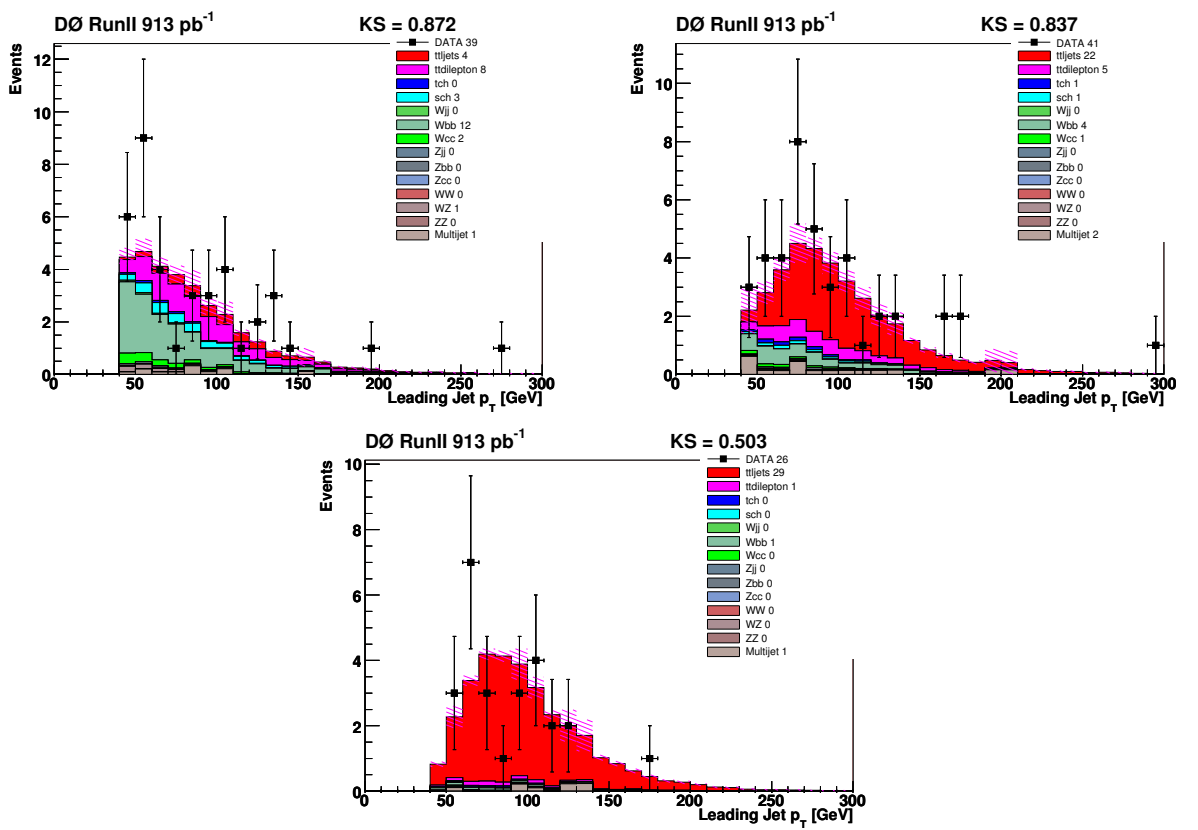

Figure C.17: Leading jet $p_{\mathrm{T}}$ distributions for the two or more tagged sample in the $e+$ jets channel. 

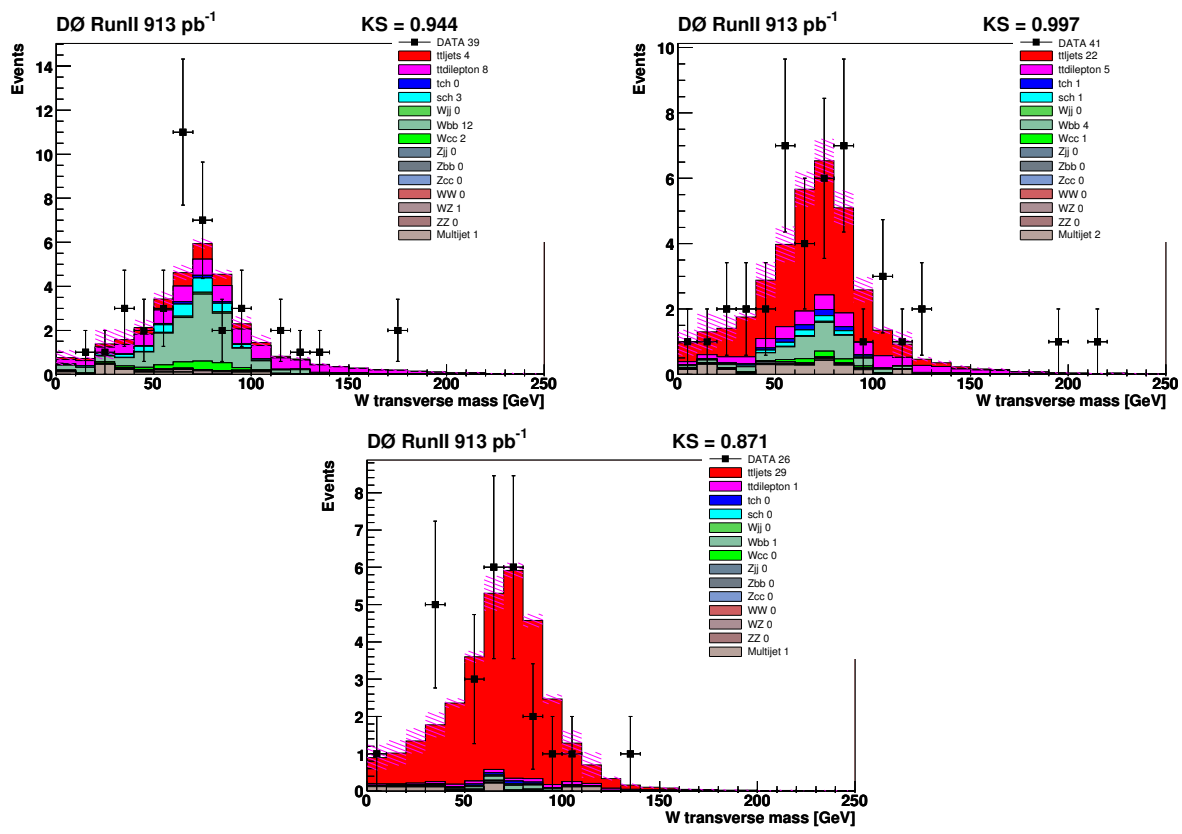

Figure C.18: The $e \nu$ transverse mass distributions for the two or more tagged sample in the $e+$ jets channel.
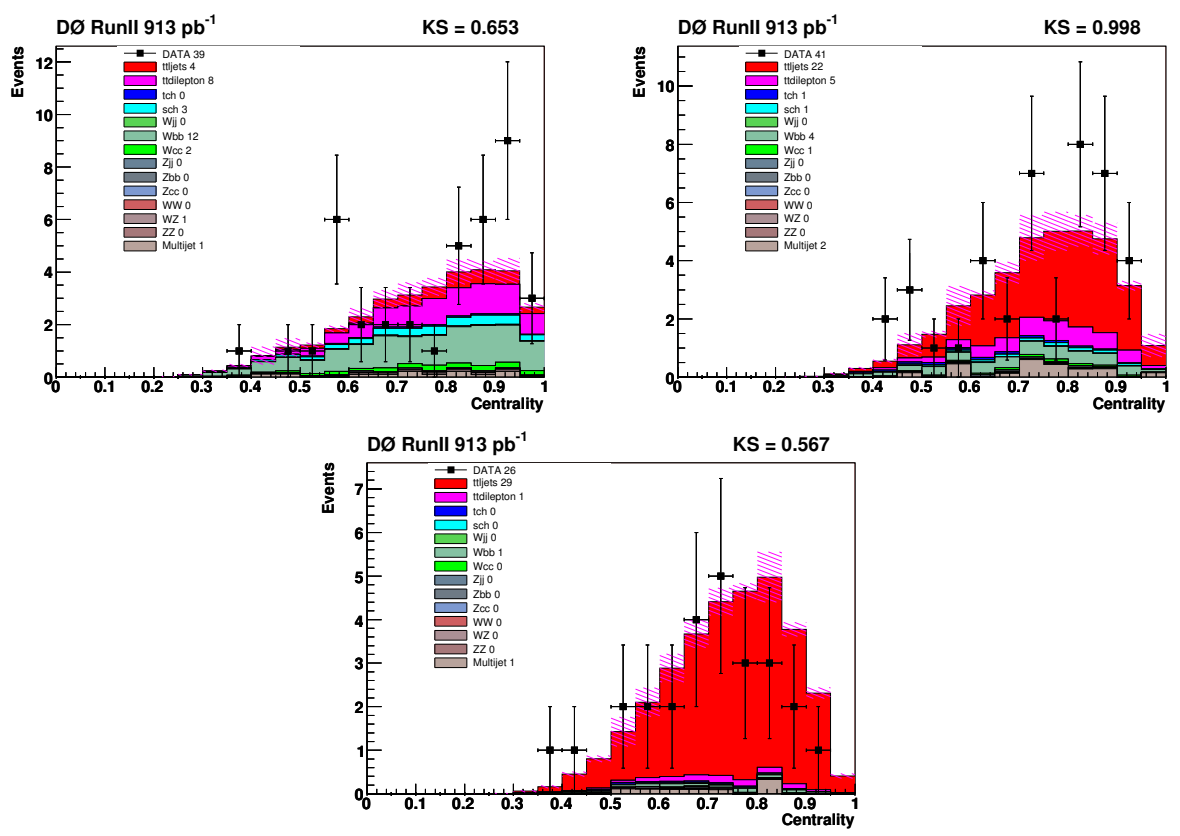

Figure C.19: Centrality distributions for the two or more tagged sample in the e+jets channel. 


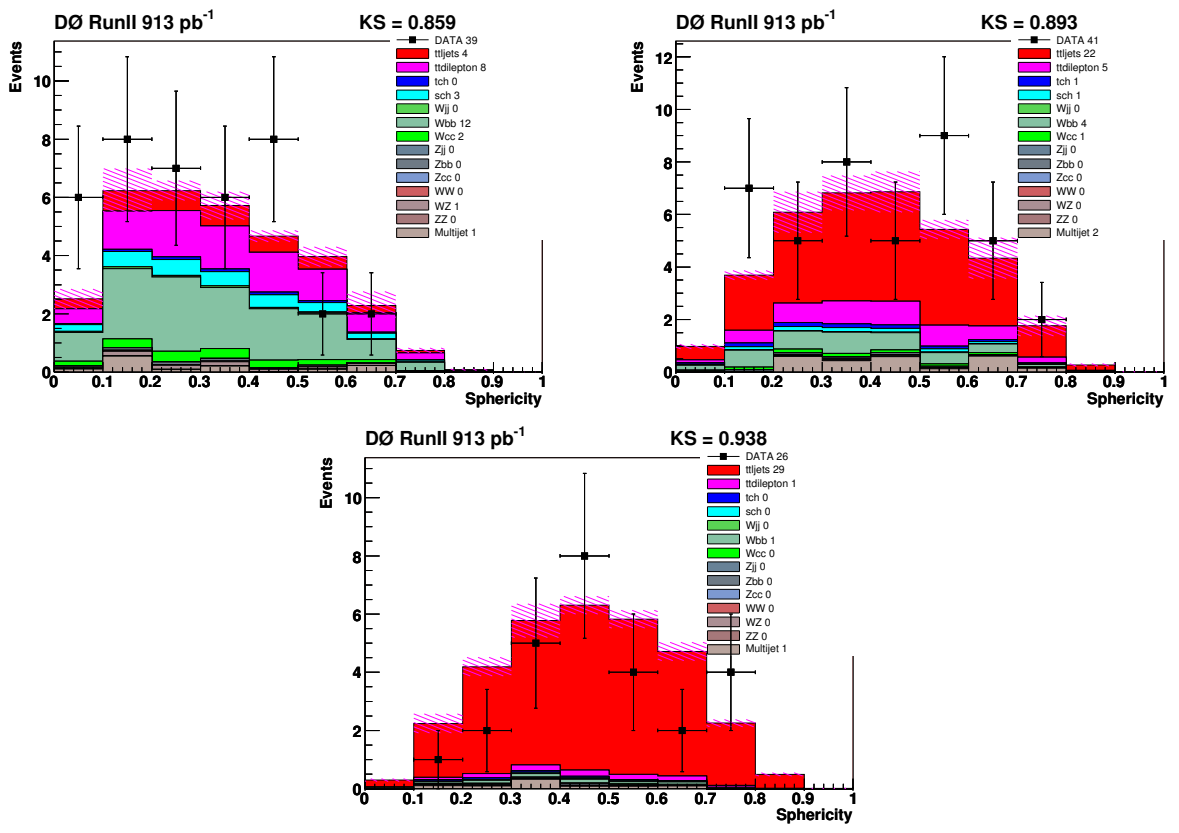

Figure C.20: Sphericity distributions for the two or more tagged sample in the $e+$ jets channel.
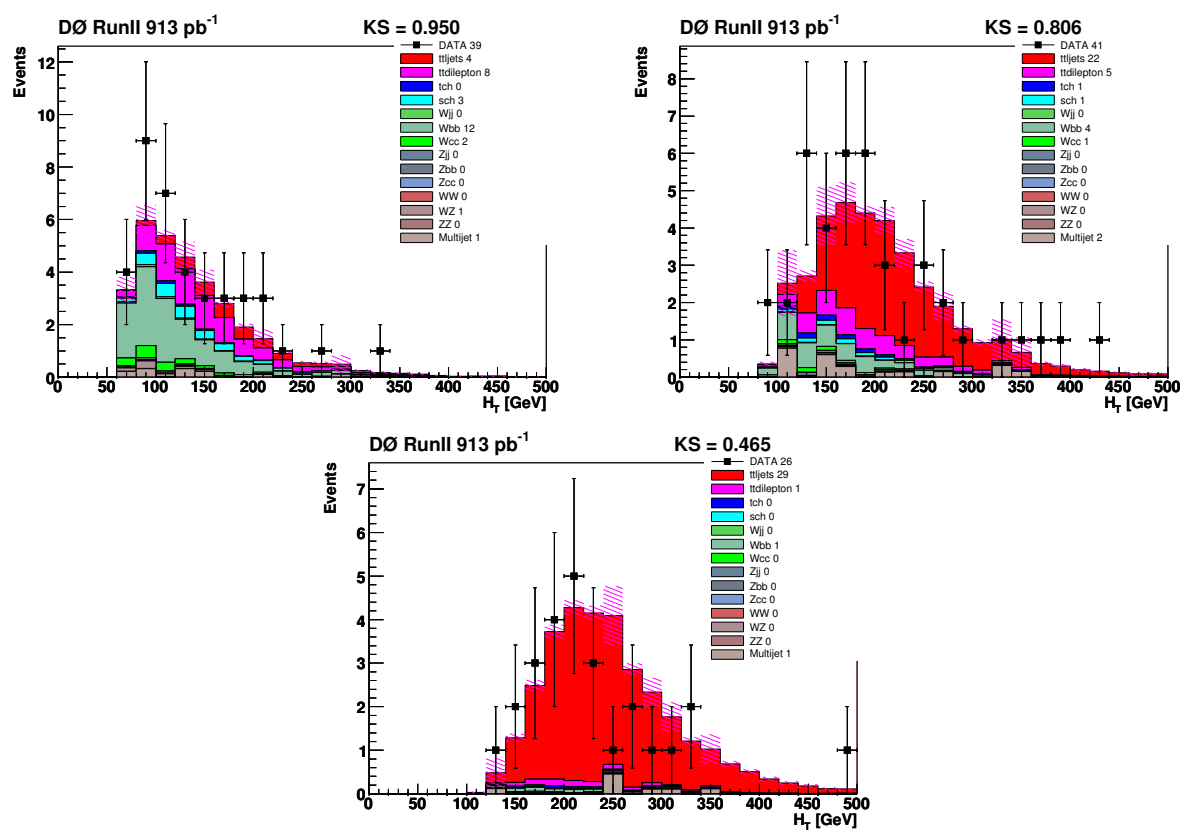

Figure C.21: $H_{T}$ distributions for the two or more tagged sample in the $e+$ jets channel. 

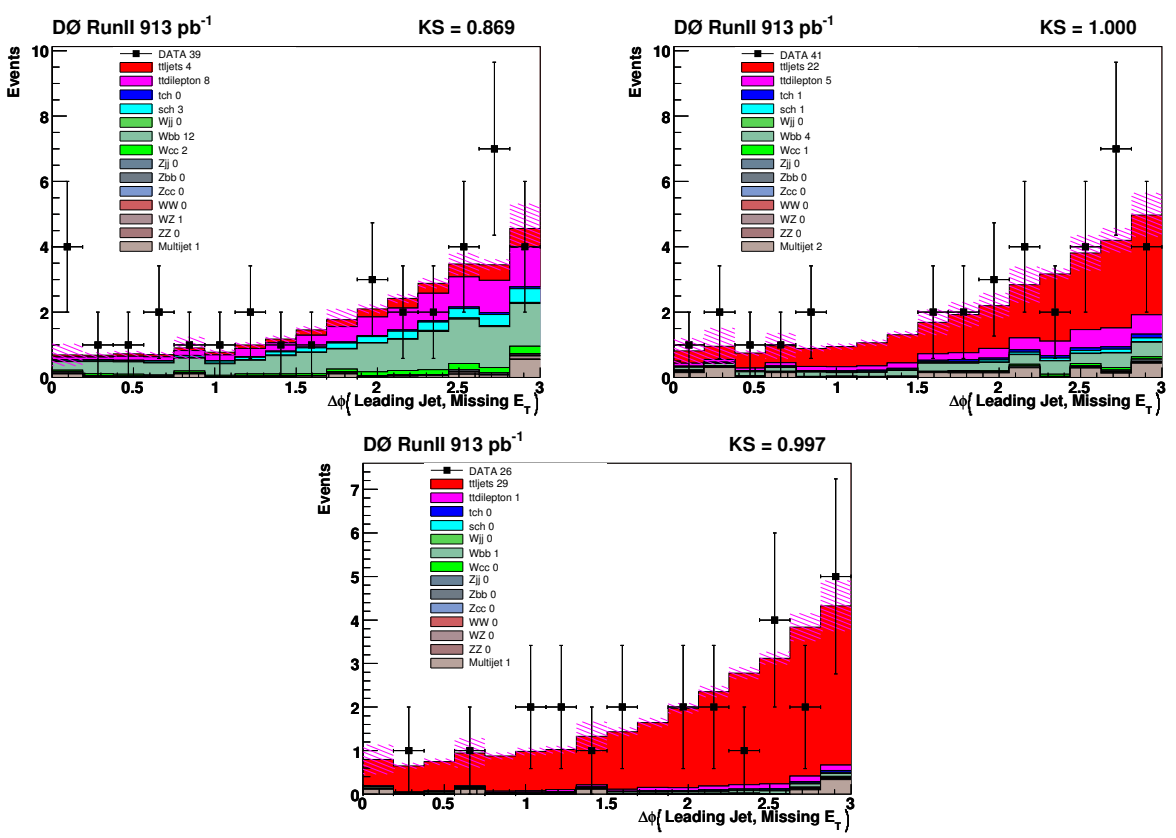

Figure C.22: $\Delta \phi\left(\right.$ leading Jet, $\left.E_{T}\right)$ distributions for the two or more tagged sample in the $e+$ jets channel.

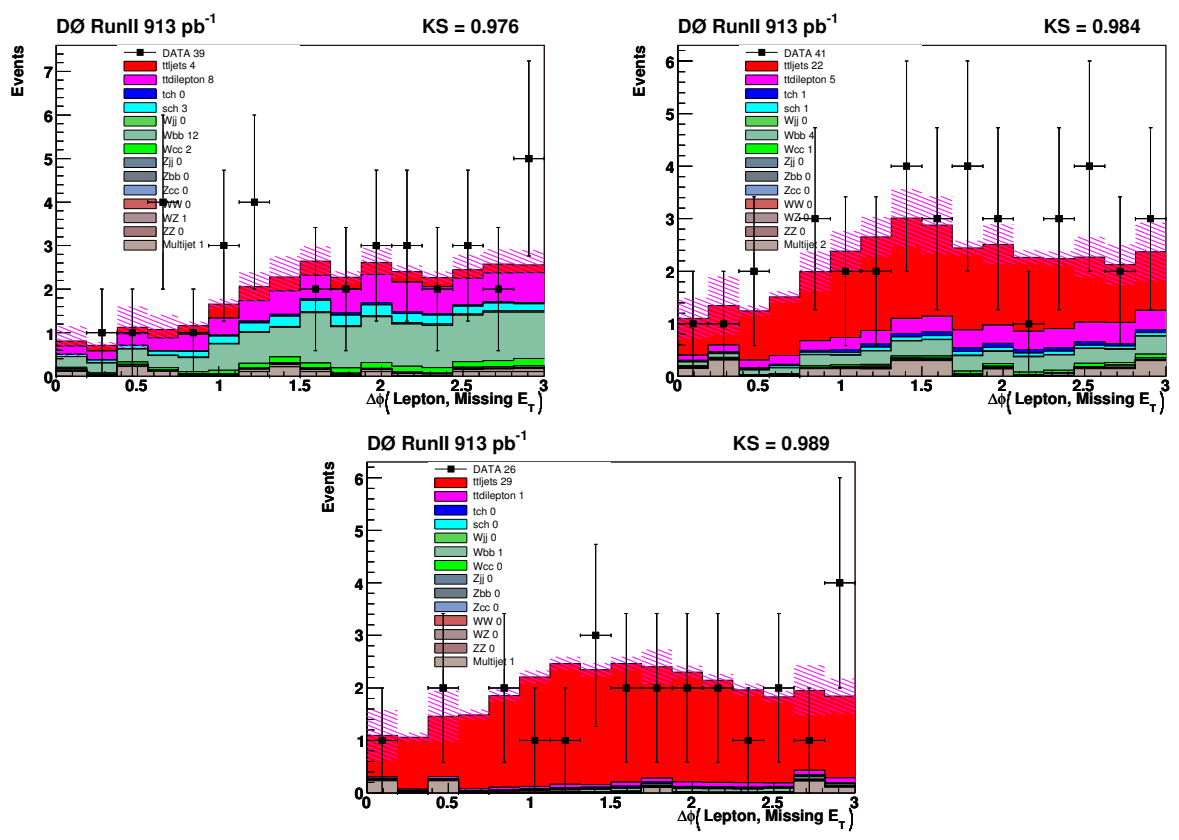

Figure C.23: $\Delta \phi\left(\right.$ electron, $\left.E_{T}\right)$ distributions for the two or more tagged sample in the $e+$ jets channel. 


\section{C.2 Control Plots for the $\mu+$ jets Channel}

\section{C.2.1 Preselection Events}
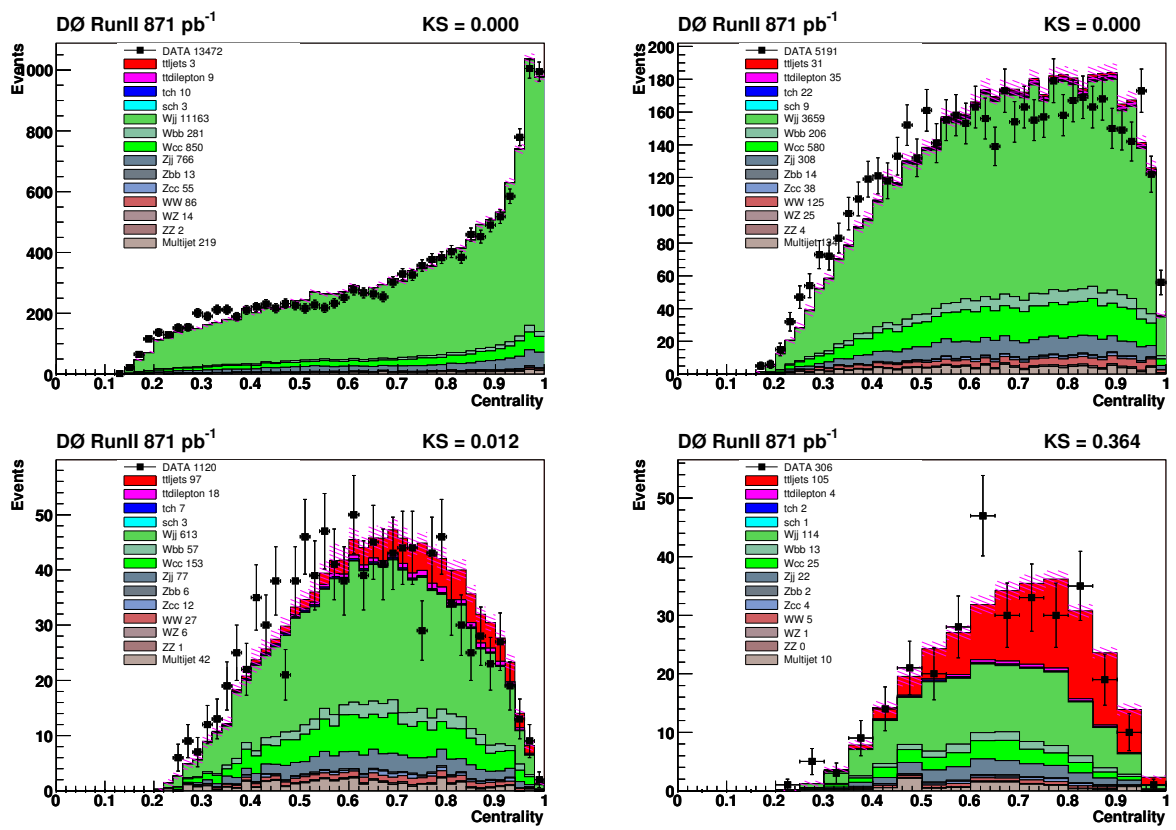

Figure C.24: Centrality distributions for the preselected sample in the $\mu+$ jets channel. 

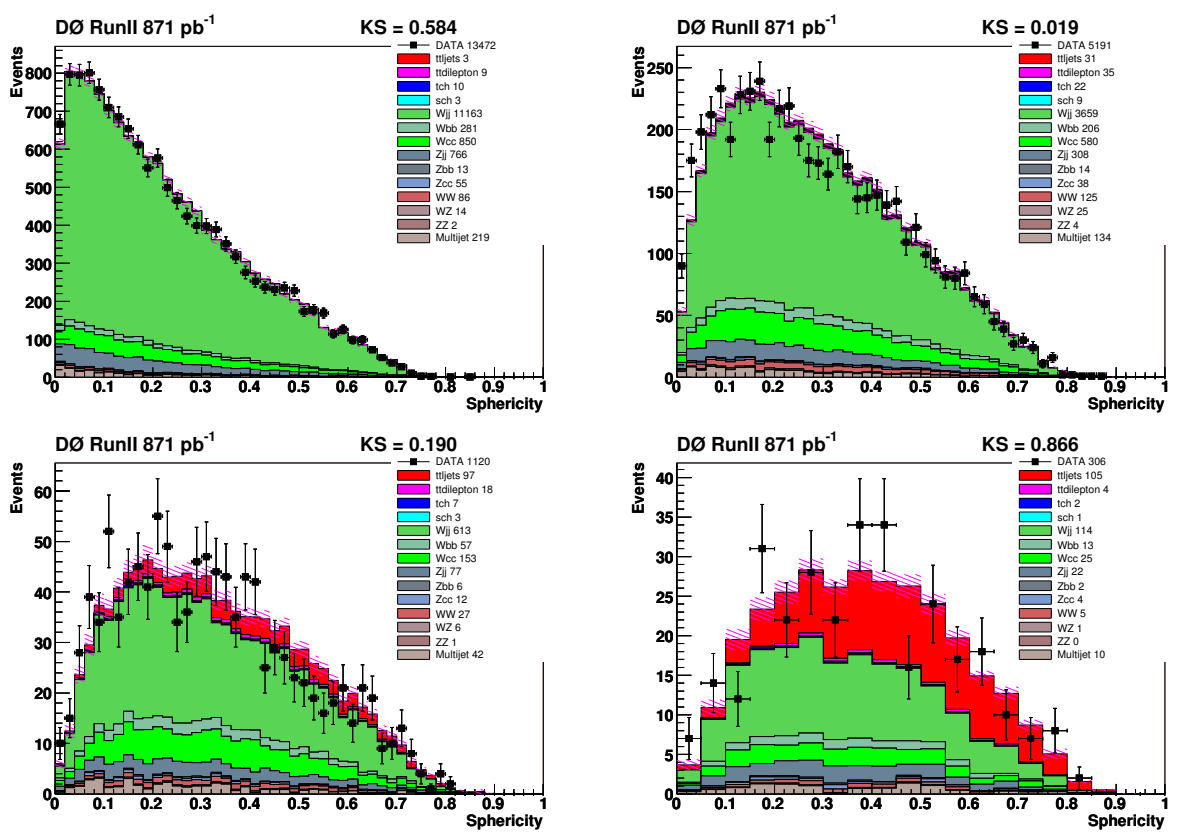

Figure C.25: Sphericity distributions for the preselected sample in the $\mu+$ jets channel.
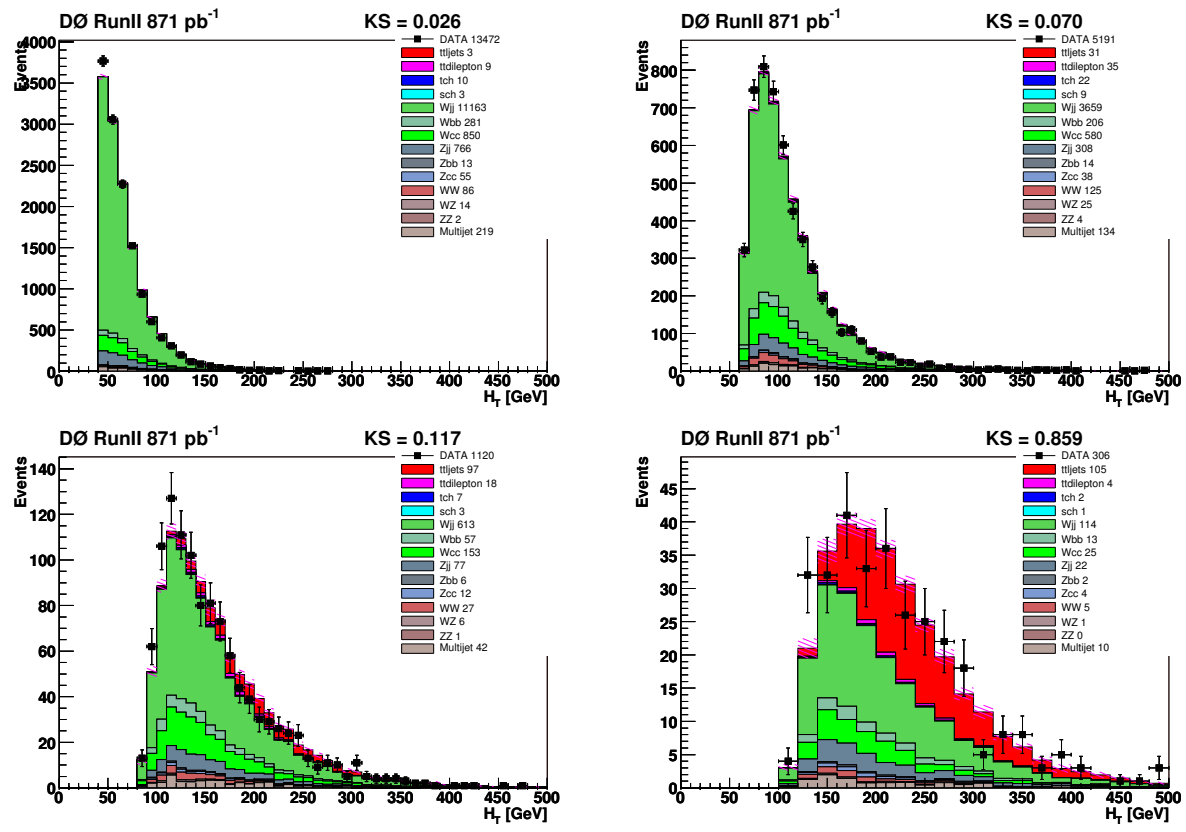

Figure C.26: $H_{T}$ distributions for the preselected sample in the $\mu+$ jets channel. 

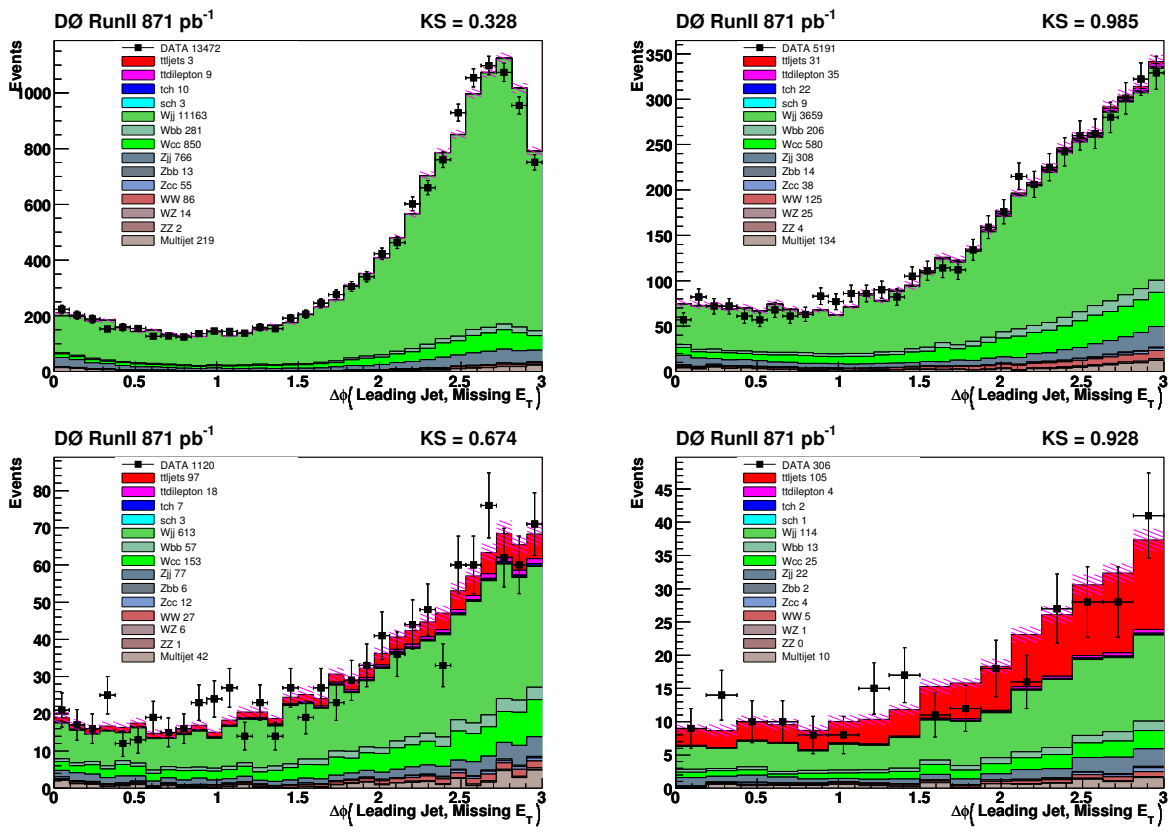

Figure C.27: $\Delta \phi\left(\right.$ leading Jet, $\left.\mathbb{E}_{T}\right)$ distributions for the preselected sample in the $\mu+$ jets channel.
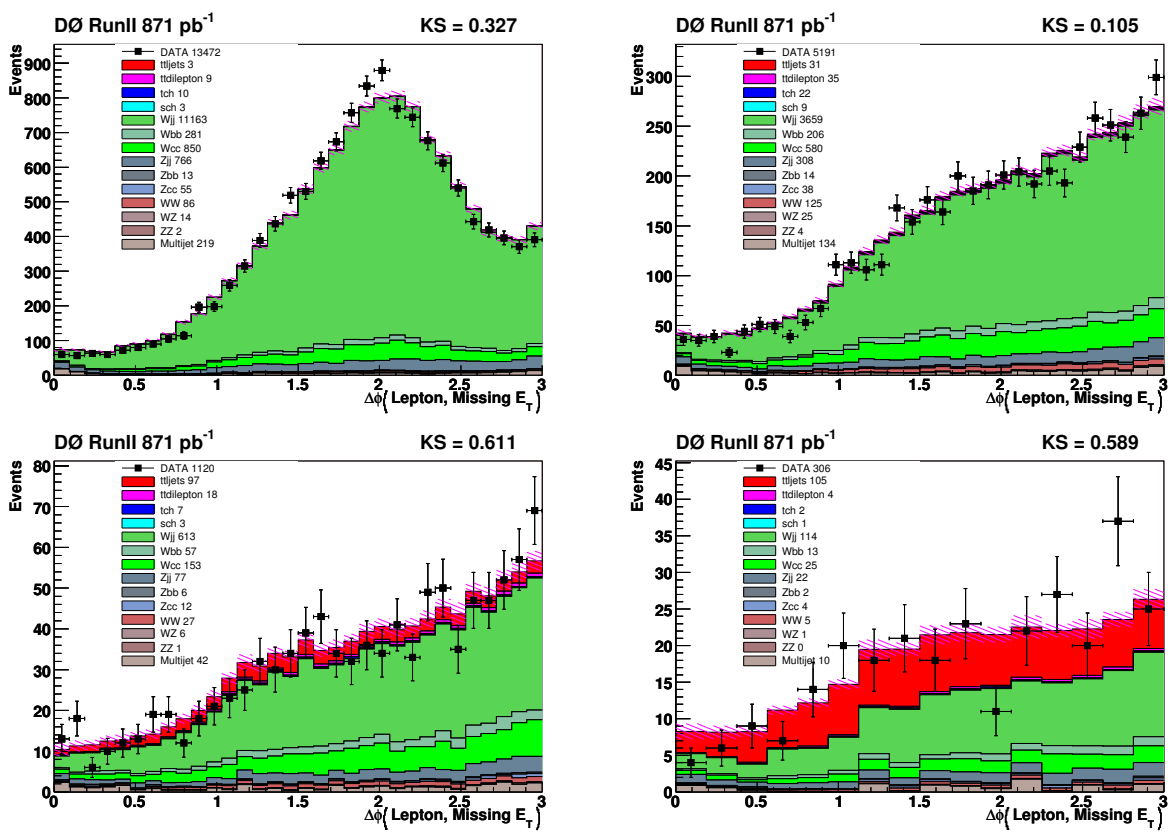

Figure C.28: $\Delta \phi\left(\right.$ muon,$\left.E_{T}\right)$ distributions for the preselected sample in the $\mu+$ jets channel. 


\section{C.2.2 Exactly One b-tagged Events}
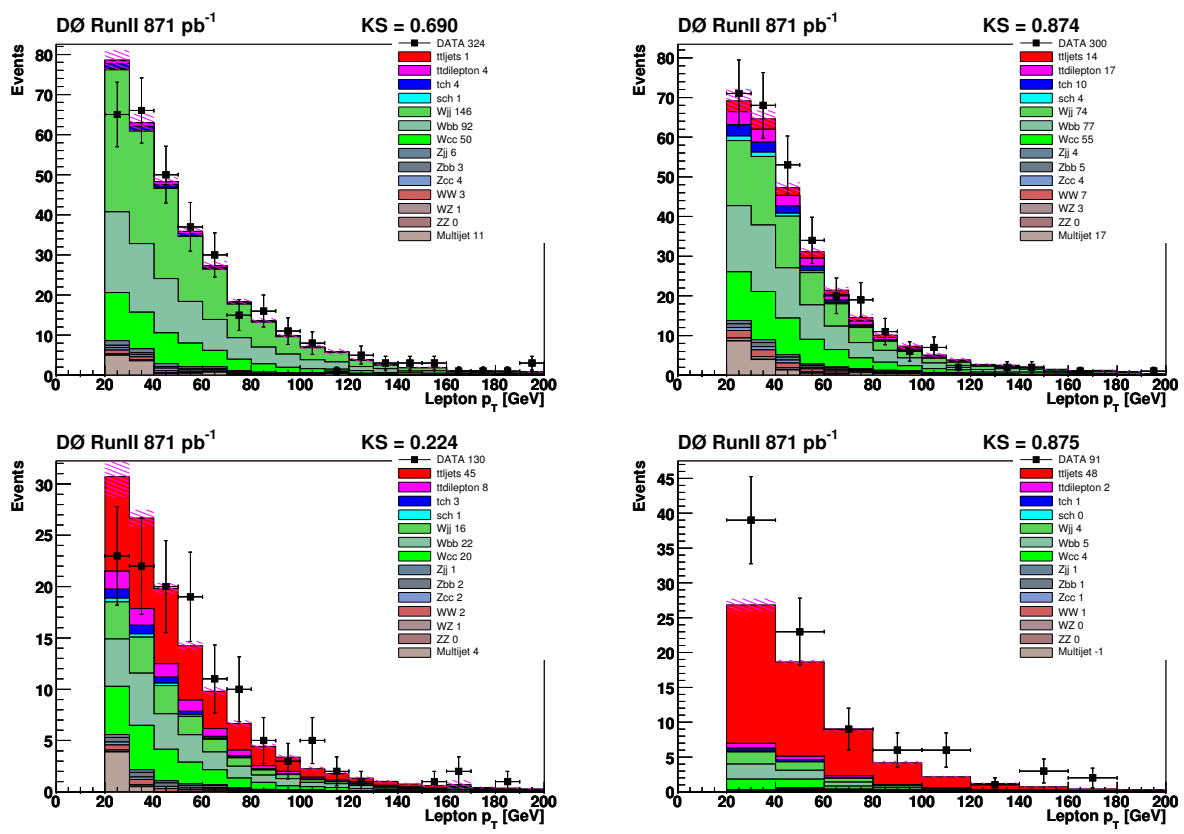

Figure C.29: Muon $p_{\mathrm{T}}$ distributions for the exactly one tagged sample in the $\mu+$ jets channel. 

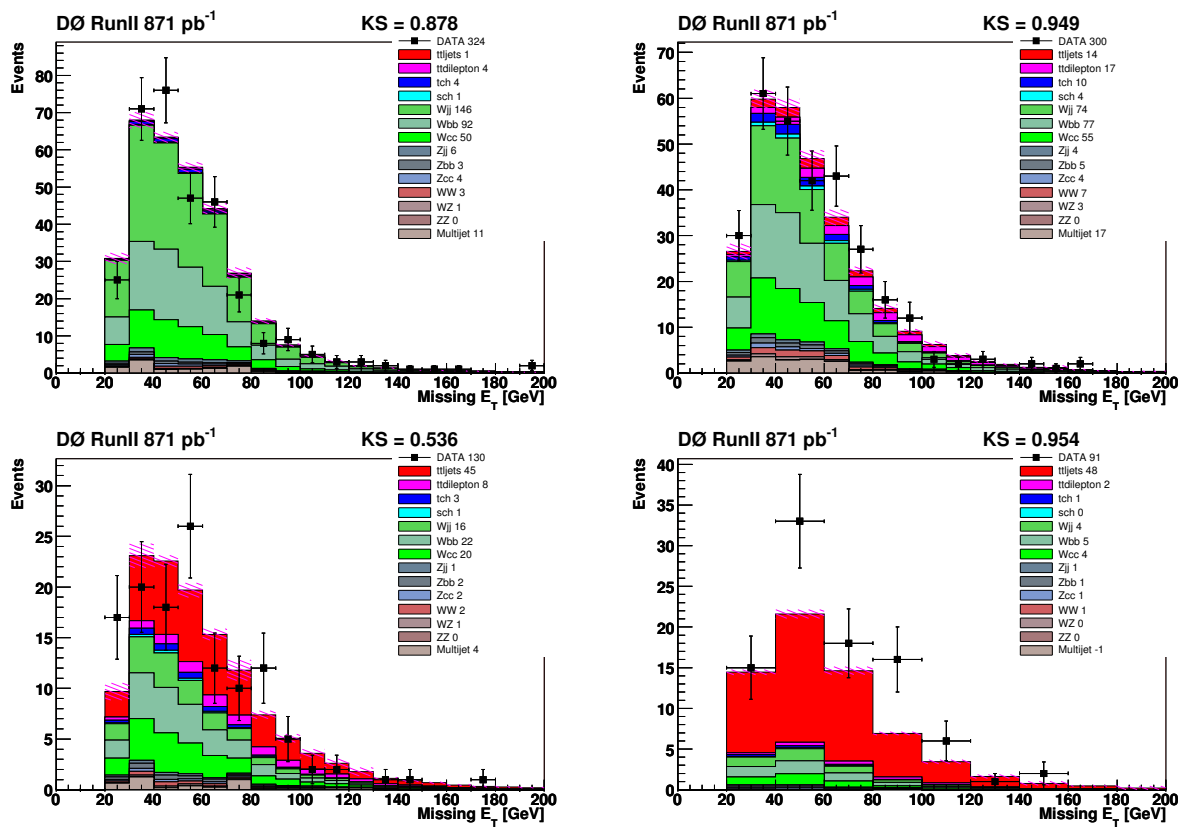

Figure C.30: $\mathbb{E}_{T}$ distributions for the exactly one tagged sample in the $\mu+$ jets channel.
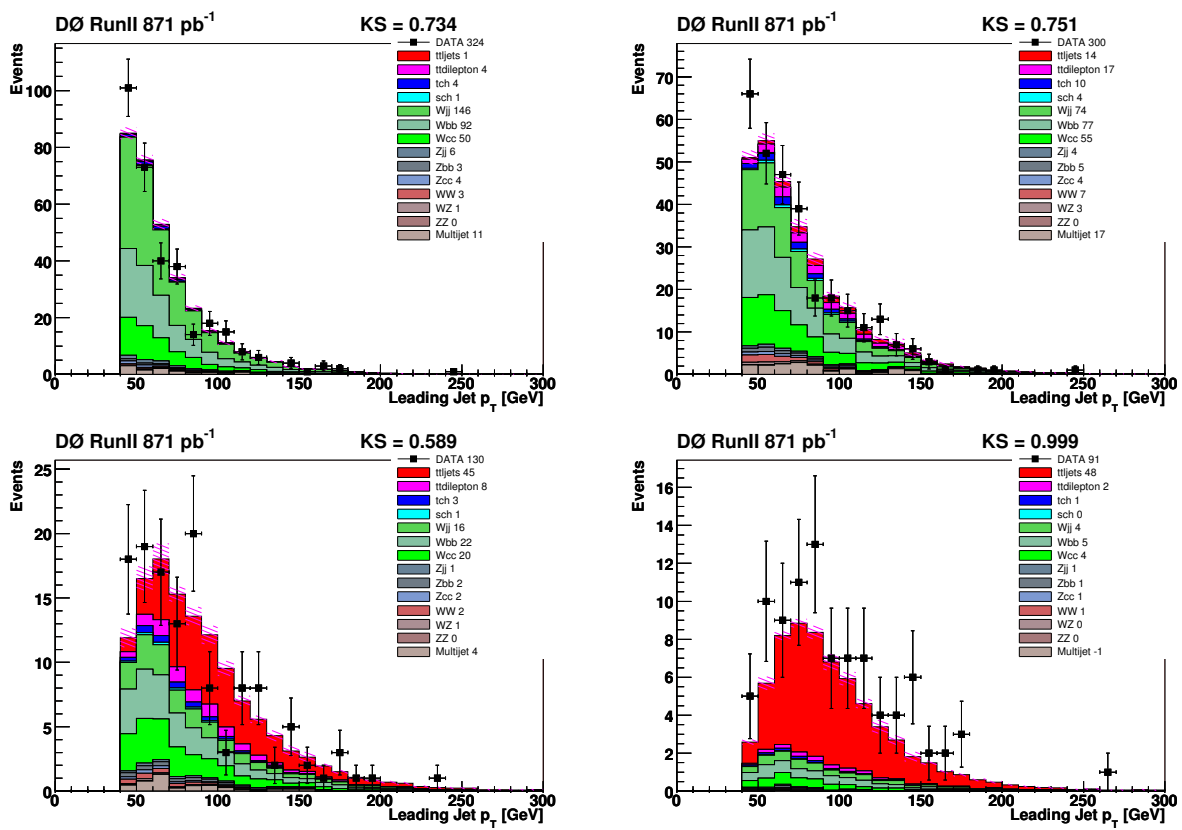

Figure C.31: Leading jet $p_{\mathrm{T}}$ distributions for the exactly one tagged sample in the $\mu+$ jets channel. 

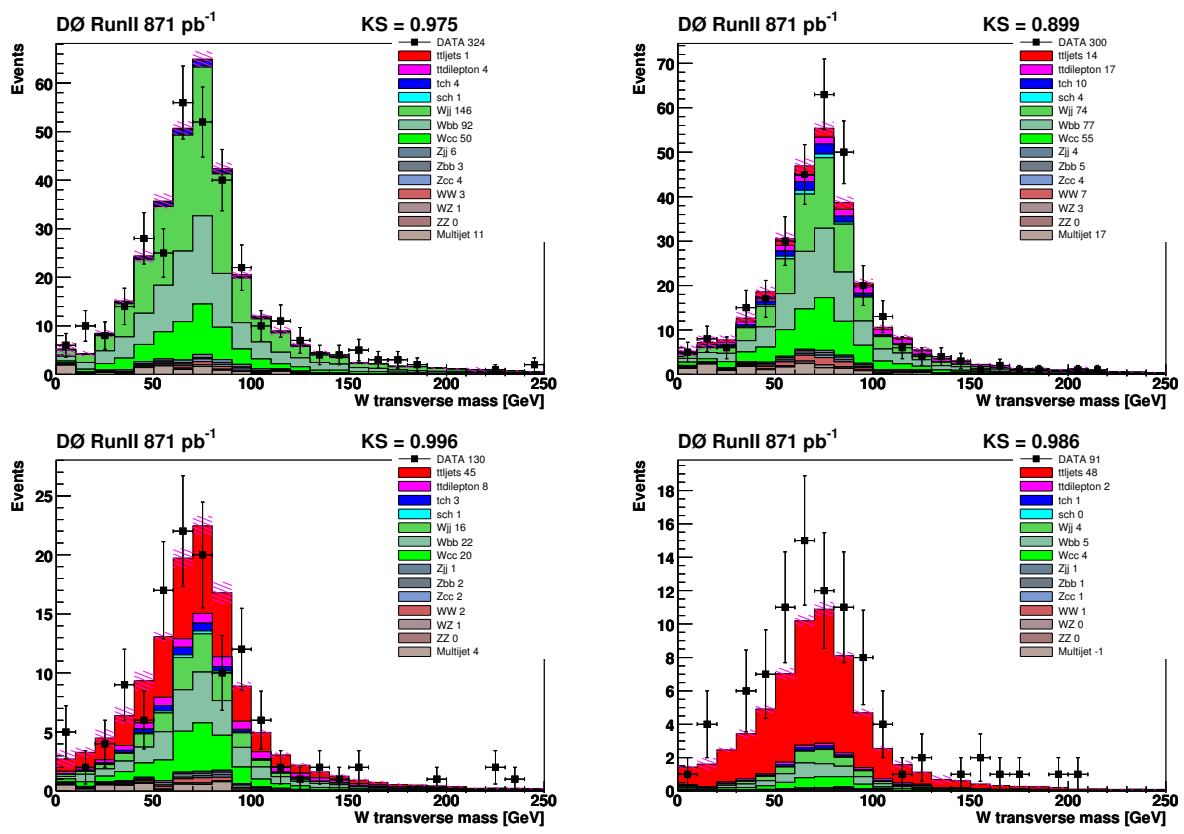

Figure C.32: The $\mu \nu$ transverse mass distributions for the exactly one tagged sample in the $\mu+$ jets channel.
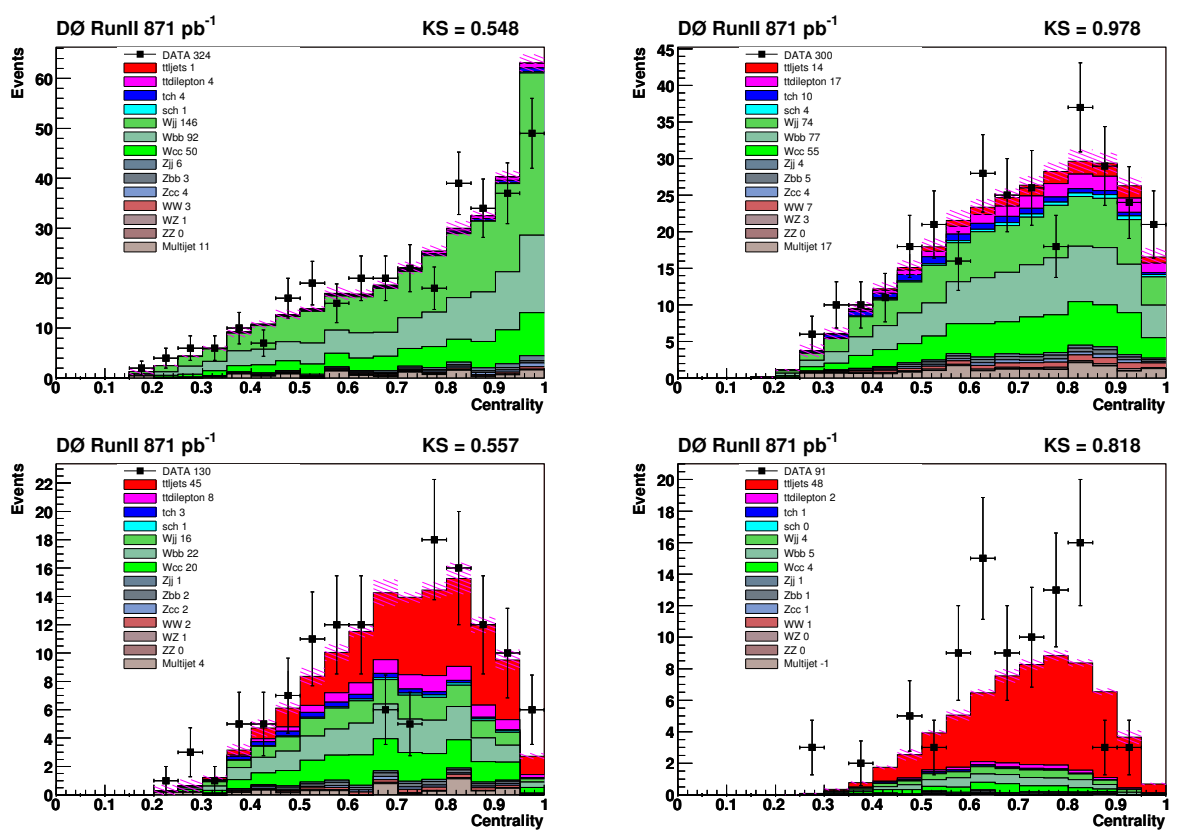

Figure C.33: Centrality distributions for the exactly one tagged sample in the $\mu+$ jets channel. 

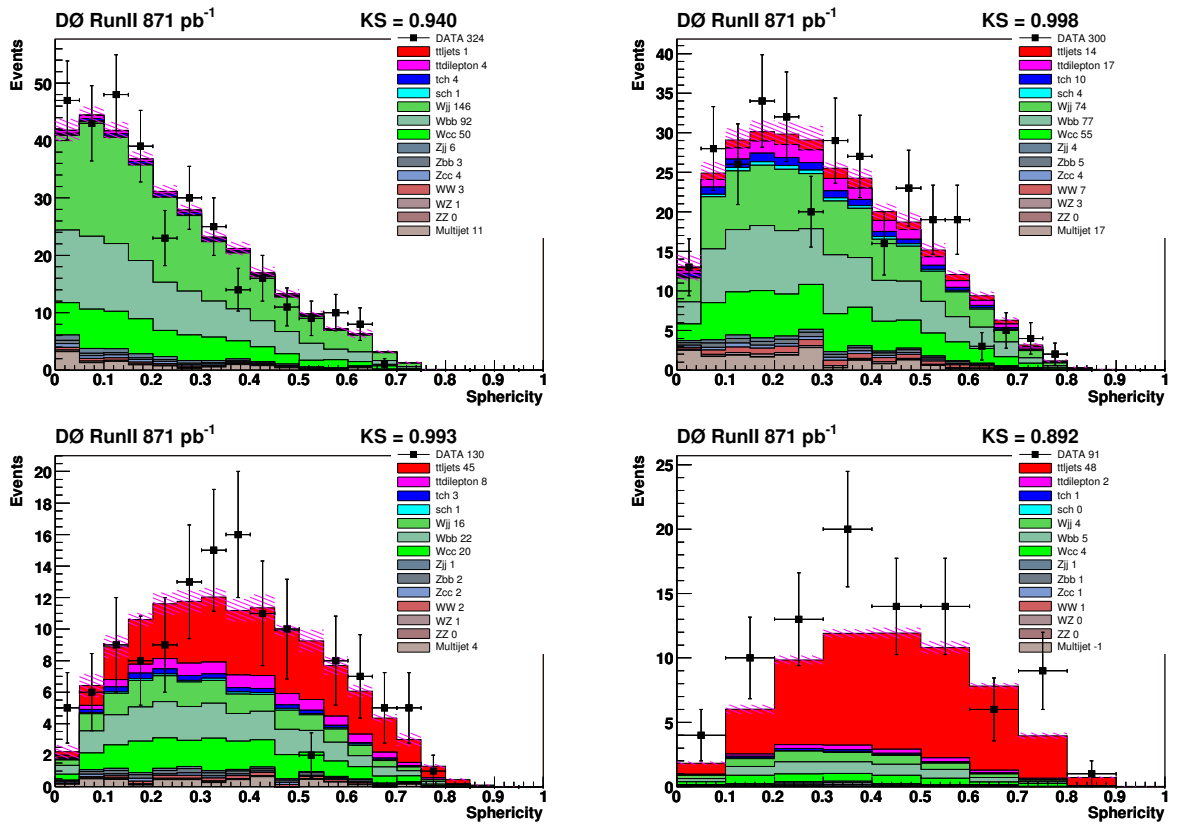

Figure C.34: Sphericity distributions for the exactly one tagged sample in the $\mu+$ jets channel.
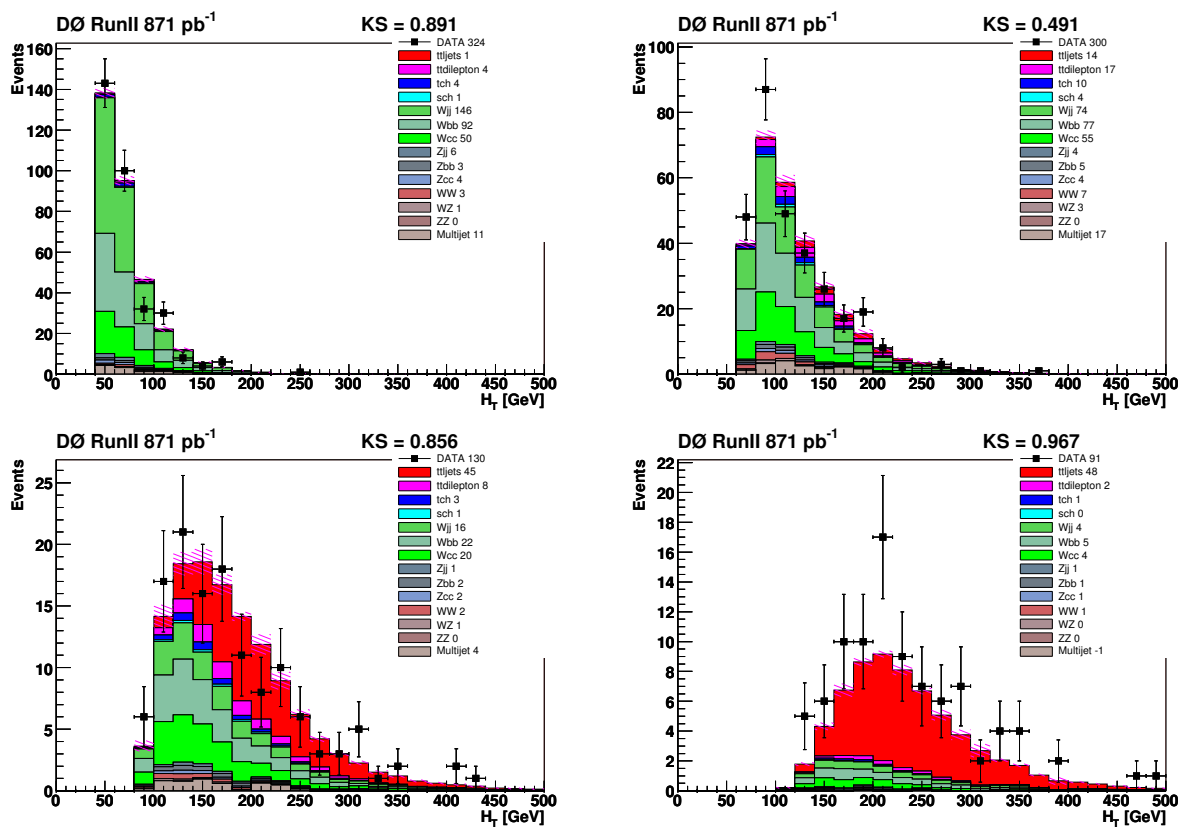

Figure C.35: $H_{T}$ distributions for the exactly one tagged sample in the $\mu+$ jets channel. 

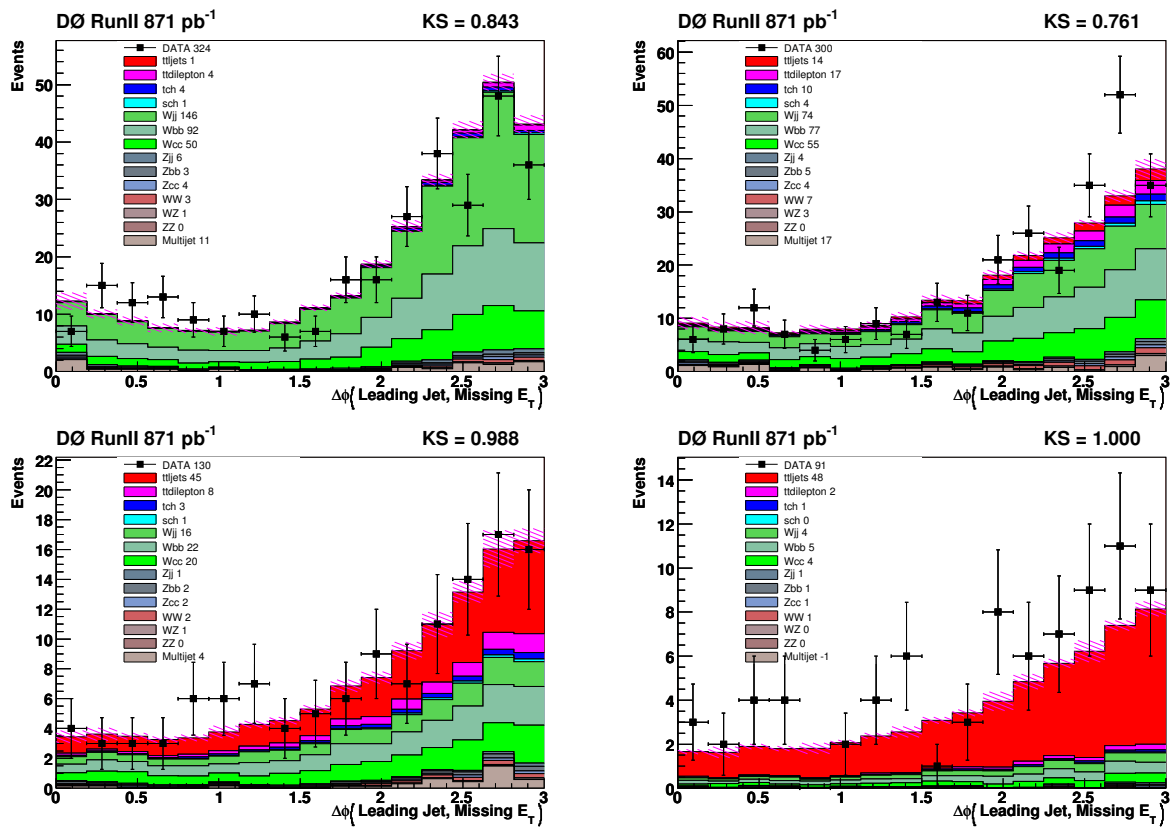

Figure C.36: $\Delta \phi$ (leading Jet, $\mathbb{E}_{T}$ ) distributions for the exactly one tagged sample in the $\mu+$ jets channel.
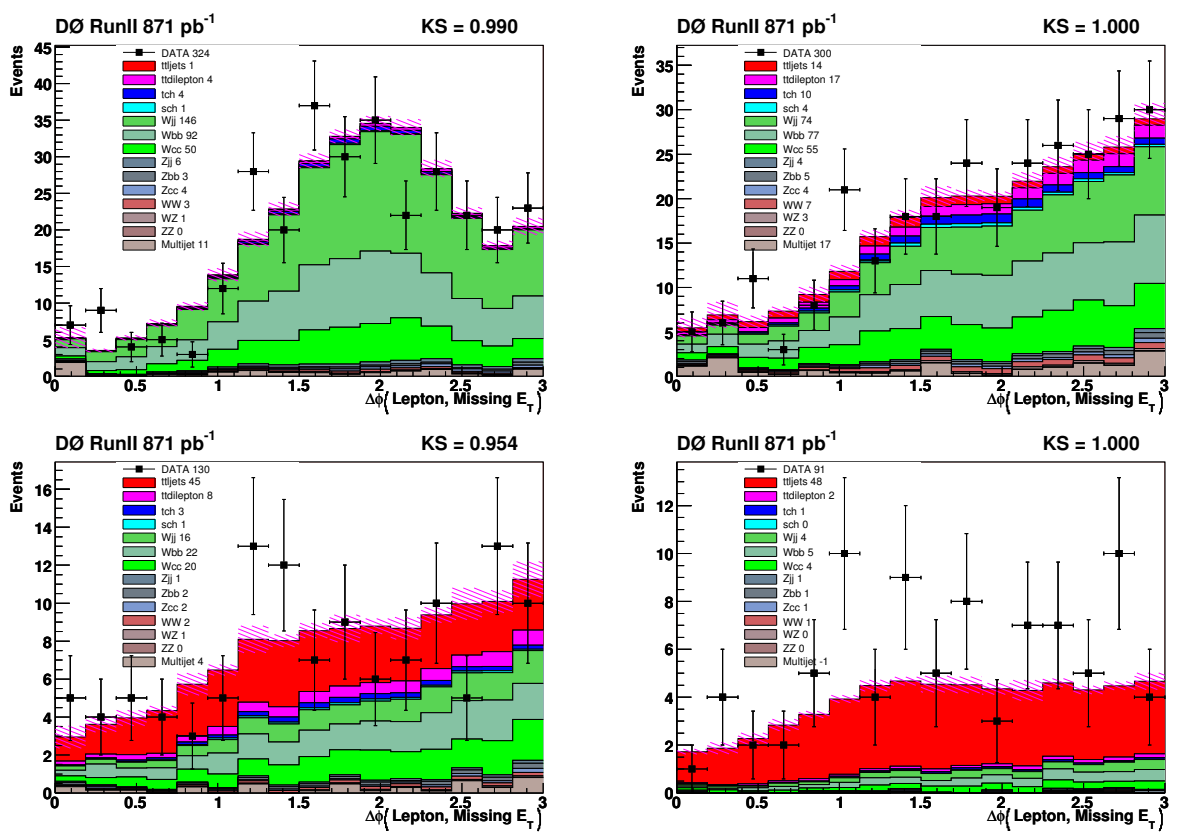

Figure C.37: $\Delta \phi\left(\right.$ muon,$\left.E_{T}\right)$ distributions for the exactly one tagged sample in the $\mu+$ jets channel. 


\section{C.2.3 Two or More b-tagged Events}

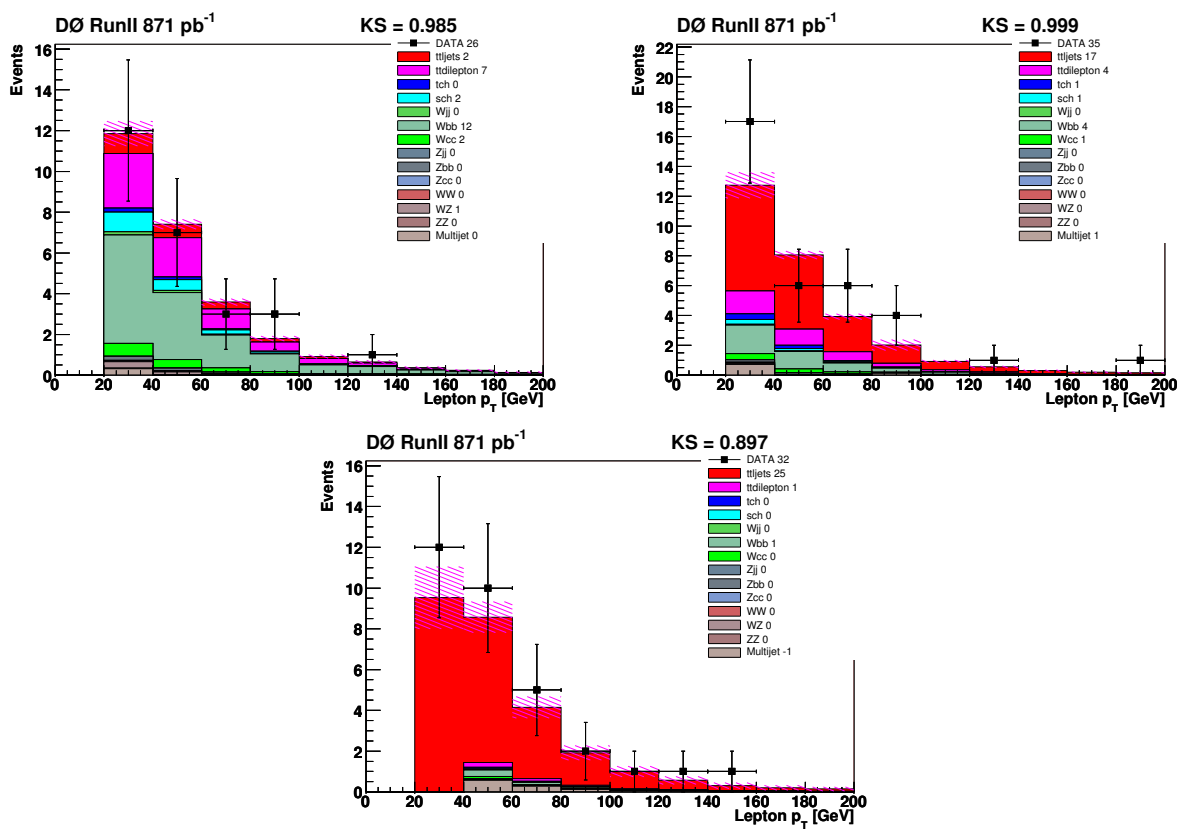

Figure C.38: Muon $p_{\mathrm{T}}$ distributions for the two or more tagged sample in the $\mu+$ jets channel. 

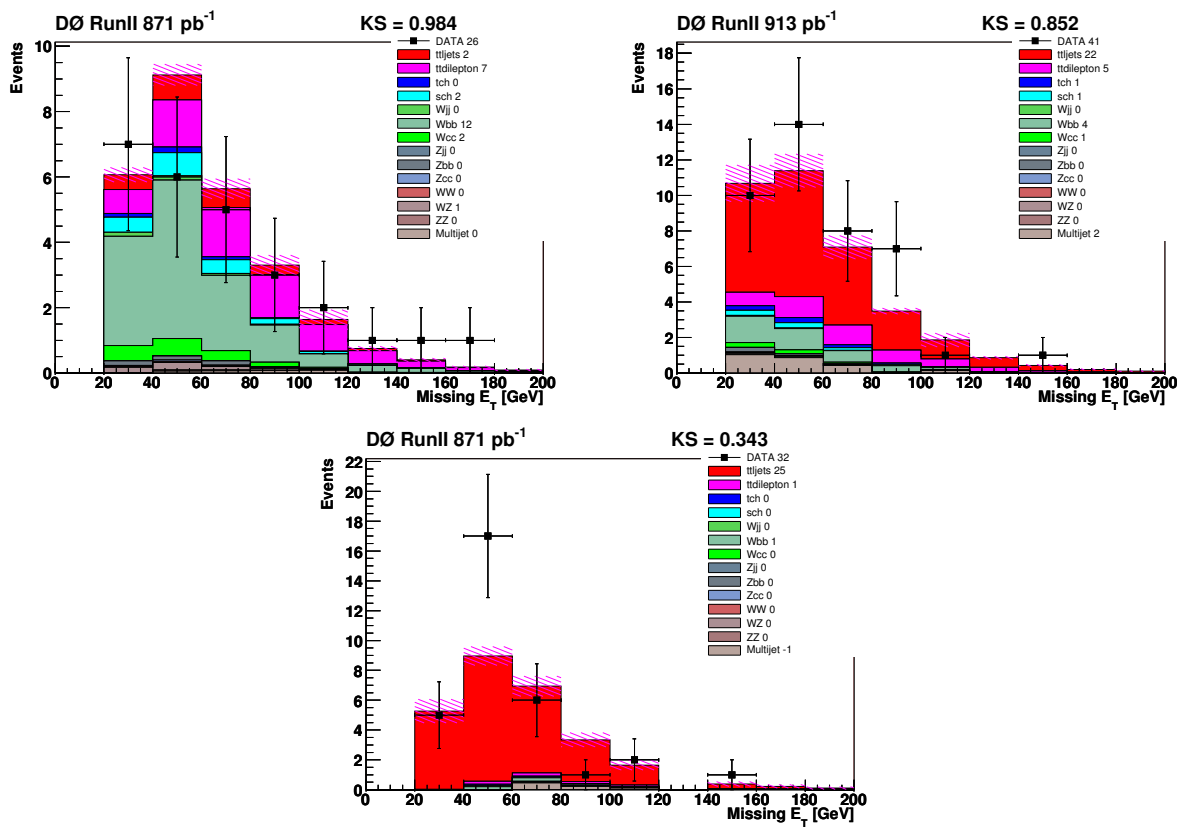

Figure C.39: $\mathbb{F}_{T}$ distributions for the two or more tagged sample in the $\mu+$ jets channel.

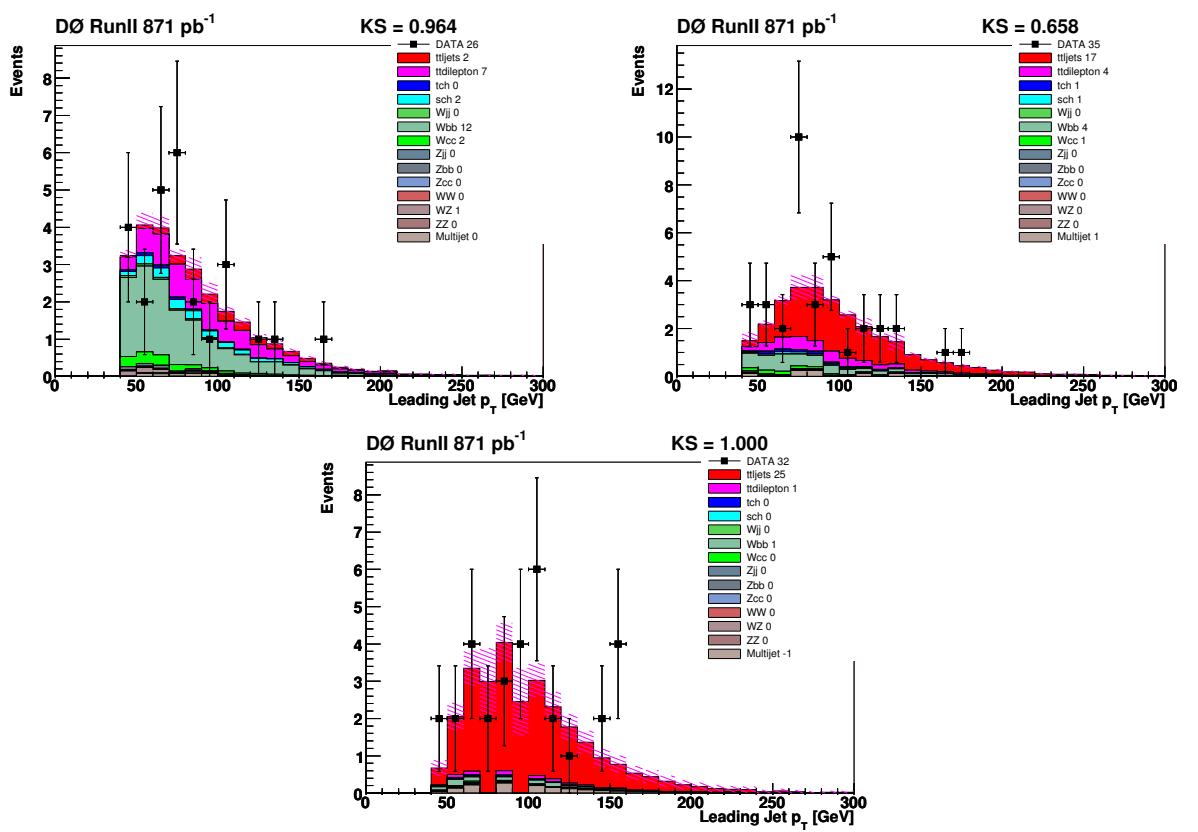

Figure C.40: Leading jet $p_{\mathrm{T}}$ distributions for the two or more tagged sample in the $\mu+$ jets channel. 

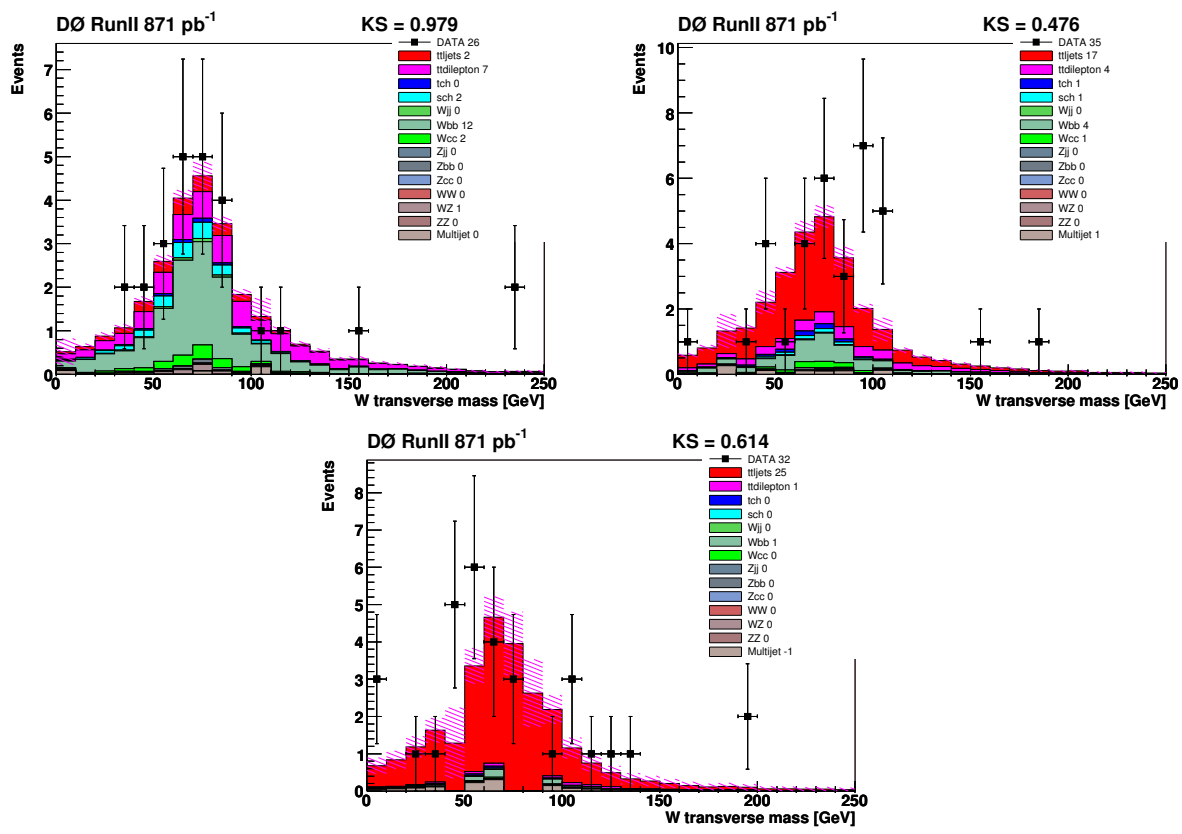

Figure C.41: The $\mu \nu$ transverse mass distributions for the two or more tagged sample in the $\mu+$ jets channel.
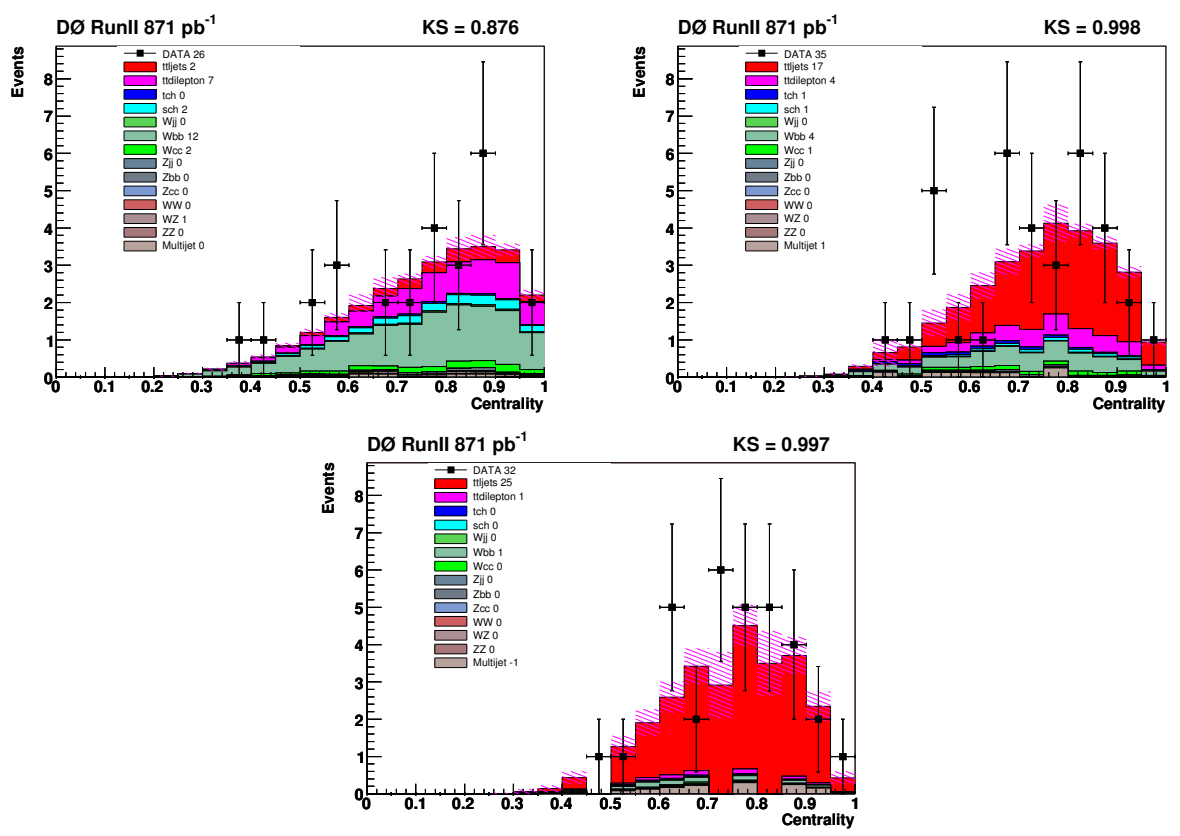

Figure C.42: Centrality distributions for the two or more tagged sample in the $\mu+$ jets channel. 

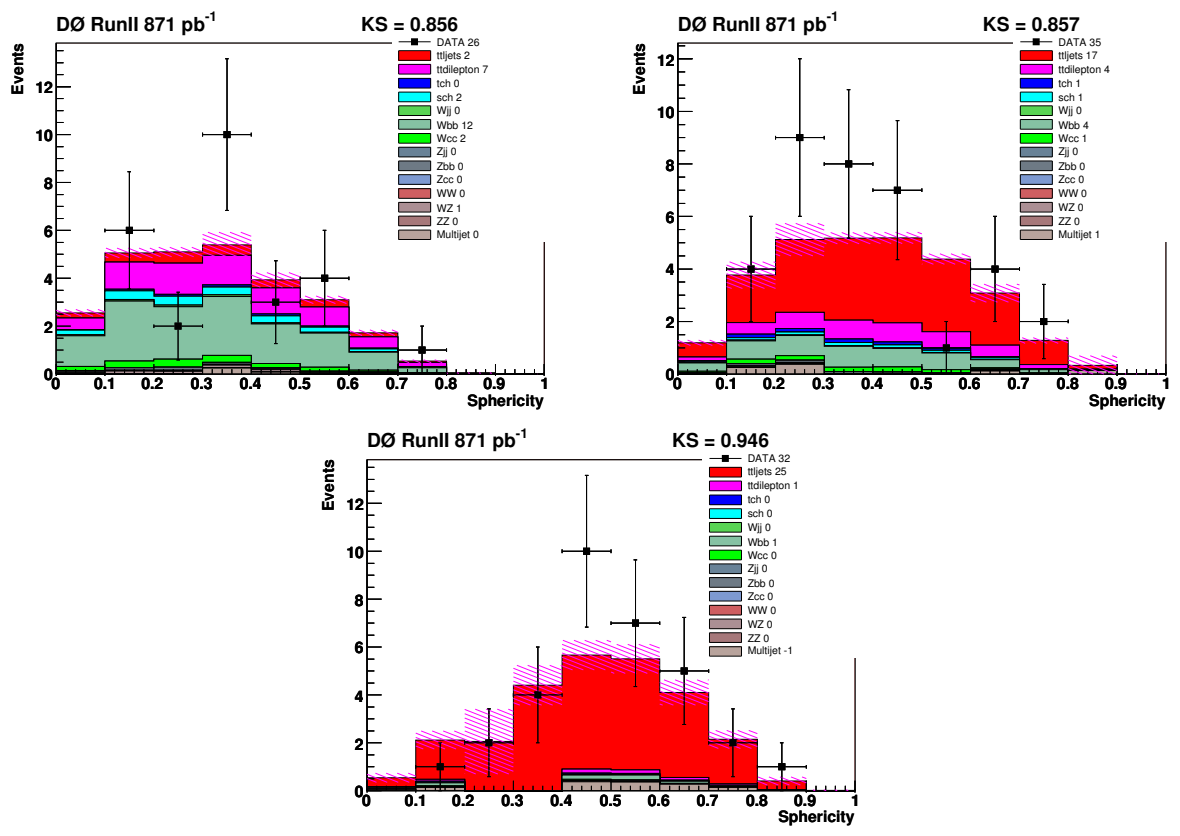

Figure C.43: Sphericity distributions for the two or more tagged sample in the $\mu+$ jets channel.
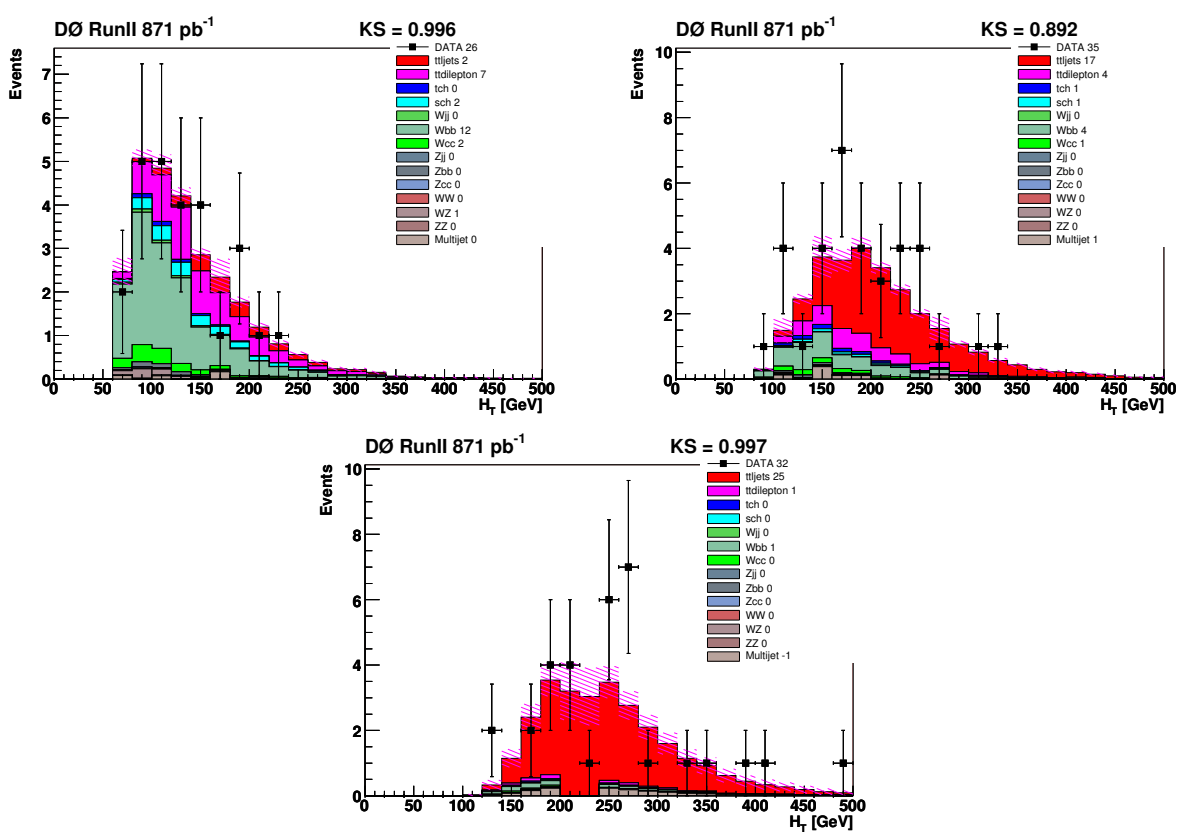

Figure C.44: $H_{T}$ distributions for the two or more tagged sample in the $\mu+$ jets channel. 

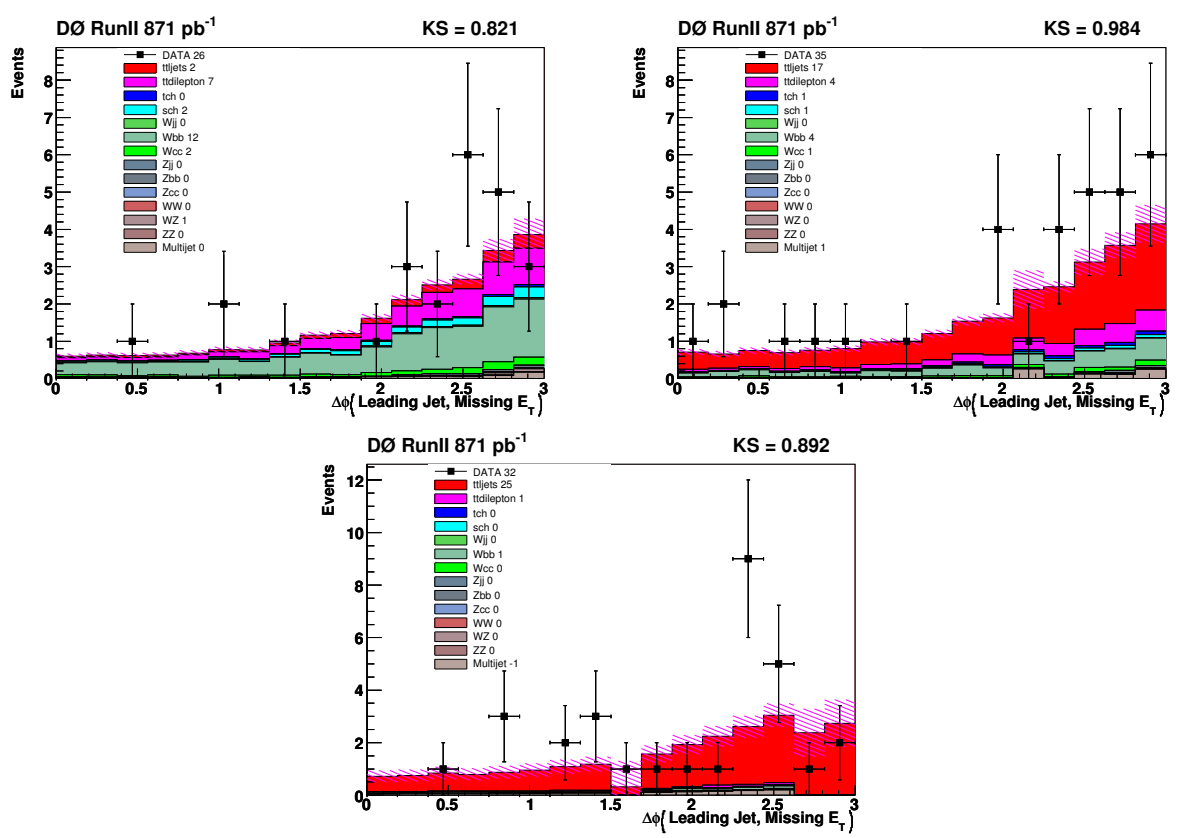

Figure C.45: $\Delta \phi$ (leading Jet, $\left.E_{T}\right)$ distributions for the two or more tagged sample in the $\mu+$ jets channel.
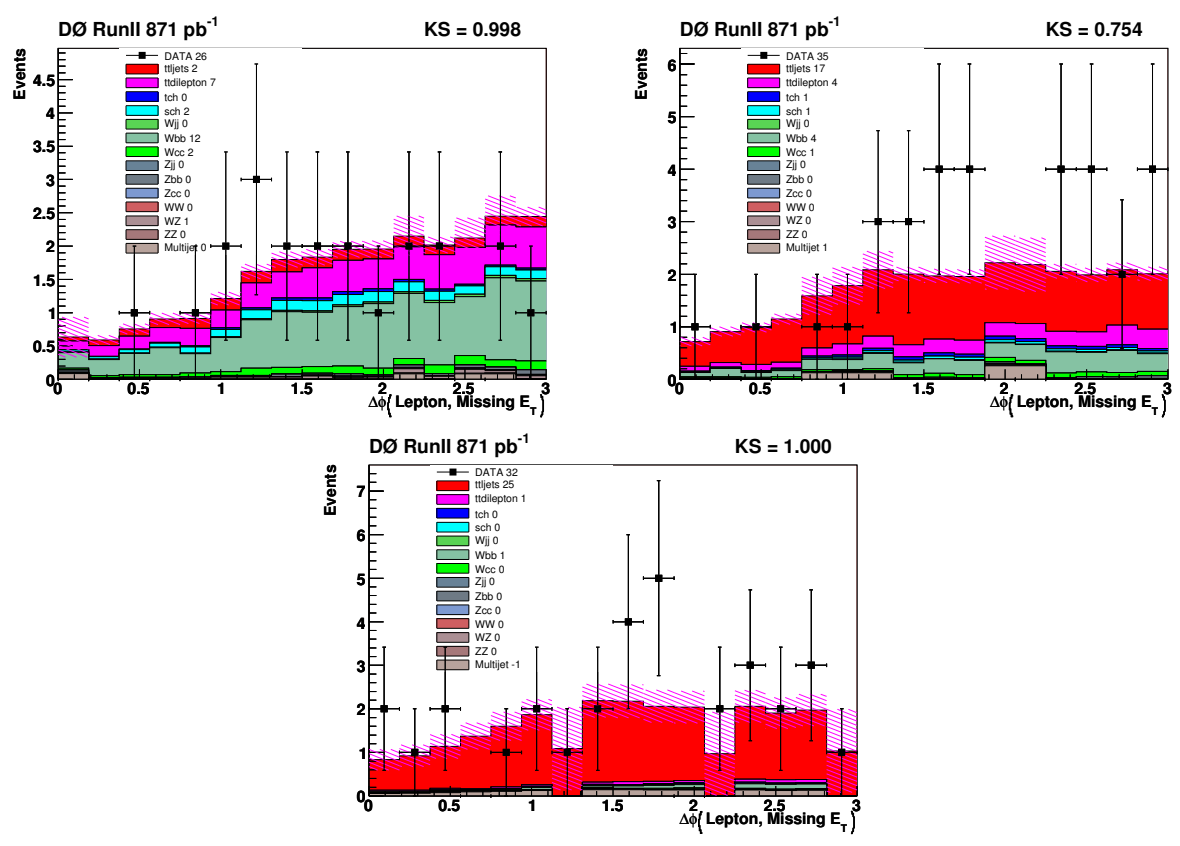

Figure C.46: $\Delta \phi\left(\right.$ muon, $\left.\mathbb{E}_{T}\right)$ distributions for the two or more tagged sample in the $\mu+$ jets channel. 


\section{Bibliography}

[1] CDF Collaboration, "Observation of the Top Quark in $p \bar{p}$ Collisions with the Collider Detector at Fermilab", Phys. Rev. Lett. 74, 2626 (1995).

[2] DØ Collaboration, "Observation of the Top Quark", Phys. Rev. Lett. 74, 2632 (1995).

[3] W.-M Yao et al., "The Review of Particle Physics 2006 Edition", J. Phys. G33, 1 (2006).

[4] Gordon Kane, "Modern Elementary Particle Physics", Addison-Wesley, Reading, MA (1993).

[5] Top Physics Group, http://www-d0.fnal.gov/Run2Physics/top/index.html.

[6] P. Nason, S. Dawson, R. K. Ellis, "The total cross section for the production of heavy quarks in hadronic collisions", FERMILAB-Pub-87/222-T (1987).

[7] N. Kidonakis and R. Vogt, "Next-to-Next-to-Leading Order Soft Gluon Corrections in Top Quark Hadroproduction,", Phys. Rev. D 68, 114014 (2003).

[8] M. Cacciari et al., "The $t \bar{t}$ Cross Section at $1.8 \mathrm{TeV}$ and $1.96 \mathrm{TeV}$ : A Study of the Systematics Due to Parton Densities and Scale Dependence", JHEP 404, 068 (2004).

[9] A.D. Martin et al., "Uncertainties of predictions from parton distributions. 1: Experimental errors", Eur. Phys. J. C 28, 455 (2003).

[10] J. Pumplin et al., "New generation of parton distributions with uncertainties from global QCD analysis" JHEP 0207, 012 (2002). 
[11] The Tevatron Electroweak Working Group for the CDF and DØ Collaborations, "Combination of CDF and DØ Results on the Mass of the Top Quark", FERMILAB-TM-2403-E.

[12] Fermi National Accelerator Laboratory (Fermilab) Homepage, http://www.fnal.gov.

[13] Accelerator Divison Homepage, http://www-bd.fnal.gov/public.

[14] http://www-bd.fnal.gov/public/proton.html.

[15] http://www-bd.fnal.gov/public/antiproton.html.

[16] http://www-bd.fnal.gov/public/maininj.html.

[17] Luminosity at Tevatron, http://www-bdnew.fnal.gov/operations/lum/lum.html.

[18] V.M. Abazov et al. (DØ Collaboration), "The Upgraded DØ Detector", Nucl. Instrum. and Methods A 565, 463-537 (2006).

[19] A. Khanov, "HTF: histogramming method for finding tracks. The algorithm description.", DØ internal note 3778.

[20] G. Borissov, "Status of D $\varnothing$ Track Reconstruction", presented at All D $\varnothing$ Meeting, Feb. 14 2003.

[21] G. Borissov, "Ordering a Chaos or... Technical Details of AA Tracking", presented at All DØ Meeting, Feb. 282003.

[22] A. Schwartzman, C. Tully, "Primary Vertex Reconstruction by Means of Adaptive Vertex Fitting", DØ internal note 4918.

[23] J. Hays et al., "Single electron efficiencies in p17 data and monte-carlo using d0correct from Release p18.05.00", DØ internal note 5105.

[24] P. Calfayan et al., "Muon Identification Certification for p17 data", DØ internal note 5157.

[25] J. Vlimant et al., "Technical description of the T42 Algorithm for the calorimeter noise suppression", DØ internal note 4146. 
[26] G. Blazey et al., "Run II Jet Physics", DØ internal note 3750.

[27] A. Harel, "Jet ID Optimization", DØ internal note 4919.

[28] http://www-d0.fnal.gov/phys_id/jes/public/plots_v7.1/.

[29] JES Group, "Jet Energy Scale Determination at DØ Run II", DØ internal note 5382.

[30] S. Calvet et al., "Towards MissingET Certification and Unclustered Energy Studies", DØ internal note 4927.

[31] R. Demina, A. Khanov, F. Rizatdinova, "b-Tagging with Counting Signed Impact Parameter Method", DØ internal note 4049.

[32] D. Bloch, B. Clement, "Update of the JLIP b-tagger Performance in p14/pass2 with Jes 5.3", DØ internal note 4824.

[33] D. Boline, L. Feligioni, M. Narain, "Update on b-quark jet identification with Secondary Vertex reconstruction using DØ reco version p14-Pass2 Samples", DØ internal note 4796.

[34] M. Anastasoaie, S. Robinson, T. Scanlon, "Performance of the NN b-tagging Tool on p17 Data", DØ internal note 5213.

[35] K. Hanagaki, J. Kasper, "Identification of b-jet by Soft Muon", DØ internal note 4867.

[36] H.D. Yoo, R. Partridge, "A Soft Muon Tagger with Neural Network on p17 Data", DØ internal note 5557 .

[37] B. Clement et al., "SystemD or how to get signal, backgrounds and their efficiencies with real data", DØ internal note 4159.

[38] P. Bevington, D. Robinson, "Data Reduction and Error Analysis for the Physical Sciences", third edition, MC-Graw-Hill (2003).

[39] M. L. Mangano et al., ALPGEN, a Generator for Hard Multiparton Processes in Hadronic Collisions, JHEP 0307:001, 2003 
[40] D. Stump et al., Inclusive Jet Production, Parton Distributions, and the Search for New Physics, JHEP 0310:046, 2003

[41] T. Sjöstrand, L. Lonnblad and S. Mrenna, PYTHIA 6.2: PHYSICS AND MANUAL, LU-TP01-21, hep-ph/0108264 (2001).

[42] Y. Fisyak, J. Womersley, "D0gstar DØ GEANT Simulation of the Total Apparatus Response", DØ internal note 3191.

[43] http://www-d0.fnal.gov/computing/MonteCarlo/mc_gallery.html.

[44] N. Makovec, J. Grivaz, "Shifting, Smearing and Removing Simulated Jets", DØ internal note 4914.

[45] http://www-d0.hef .kun.nl///fullAgenda.php?ida=a052073.

[46] Common Sample Group, http://www-d0.fnal.gov/Run2Physics/cs/index.html.

[47] DØ Top Triggers, http://www-do.fnal.gov/Run2Physics/top/d0_private/wg/triggers/triggers.html.

[48] Brendan Casey et al., "Determination of the Effective Inelastic $p \bar{p}$ Cross-Section for the DØ Luminosity Measurement Using Upgraded Readout Electronics", DØ internal note 4958.

[49] T. Andeen et al., "The DØ experiment's integrated luminosity for Tevatron Run IIa", FERMILAB-TM-2365 (2007)

[50] S. Höche et al., "Matching Parton Showers and Matrix Elements", hep-ph/0602031.

[51] Yvonne Peters et al., "Study of the W+jets heavy flavor scale factor in p17", DØ internal note 5406.

[52] E.E. Boos et al., "Method for Simulating Electroweak Top-Quark Production Events in the NLO Approximation: SingleTop Generator", Phys. Atom. Nucl. 69, 1317 (2006).

[53] E. Aguiló et al., "Search for Single Top Quark Production in $1 \mathrm{fb}^{-1}$ of Data", DØ internal note 5285 . 
[54] Shaohua Fu, Andy Haas, "A search for $Z H\left(\rightarrow e^{+} e^{-} b \bar{b}\right)$ Production with the DØ Detector in $p \bar{p}$ Collisions at $\sqrt{s}=1.96 \mathrm{TeV} ", \mathrm{D} \varnothing$ internal note 5275.

[55] Y. Arnoud et al., "Measurement of the $t \bar{t}$ Production Cross-Section at $\sqrt{s}=1.96 \mathrm{TeV}$ in the Dilepton Final States Using $1 \mathrm{fb}^{-1} "$, D $\varnothing$ internal note 5371.

[56] B. Martin et al., "Measurement of the $t \bar{t}$ production cross section at $\sqrt{s}=1.96 \mathrm{TeV}$ in the ee final state using p17 data set", DØ internal note 5386 .

[57] Data Quality Group web page:

http://www-d0.fnal.gov/computing/data_quality/d0_private/forusers.html.

The recommended tags are used: caf_dq v02-01-01, with dq_defs v2006-05-04.

[58] Marc-André Pleier, "Measurement of the Electron and Muon Fake Rates in Lepton+Jets Datasets", DØ internal note 5469.

[59] S.-J. Park, M. Begel, "Efficiency of the Data Quality Calorimeter Flags", DØ internal note 5324 .

[60] Yvonne Peters et al., "Simultaneous measurement of $\mathrm{B}(\mathrm{t} \rightarrow \mathrm{Wb}) / \mathrm{B}(\mathrm{t} \rightarrow \mathrm{Wq})$ and $\sigma(p \bar{p} \rightarrow t \bar{t}$ )$\cdot \mathrm{B}(\mathrm{t} \rightarrow \mathrm{Wb})^{2}$ with $\mathrm{p} 17$ data", $\mathrm{D} \varnothing$ internal note 5422.

[61] Ulrich Heintz, Matrix Method Uncertainties, DØ internal note 5534.

[62] I. Anghel et al., "Studies of Taggability versus number of Primary Vertices for p17 data", $\mathrm{D} \varnothing$ internal note 5240.

[63] Heidi Schellman, "The longitudinal shape of the luminous region at DØ", DØ internal note 5142.

[64] Bertrand Martin, "p17 Dielectron Selection", presented at Precision Measurements Meeting, September 24, 2007.

[65] Yvonne Peters et al., "Reweighting of the fragmentation function for the DØ Monte Carlo", DØ internal note 5325. 
[66] G. Garzon et al., "Measurement of the $t \bar{t}$ Production Cross Section in $p \bar{p}$ Collisions at $\sqrt{s}=$ 1.96TeV using Secondary Vertex b-Tagging", DØ internal note 5113.

[67] Andy Haas, "b-ID Status", presented in the DØ Conveners Meeting, May 25, 2007.

[68] Yvonne Peters et al., "Study of Monte Carlo Factorization scale for D 5373.

[69] John Anderson et al., "Upgrade of the DØ Luminosity Monitor Readout System", FERMILAB-CONF-06-470-E.

[70] B. Baldin et al., "DØ Upgrade Muon Electronics Design", IEEE Trans. on Nuclear Science, Vol.42, No.4, pp 736-742, 1995.

[71] B. Casey, C. Miao, R. Partridge, "DØ Run 2 Luminosity Monitor Vertex Board Specifications", DØ internal note 4857.

[72] V. Bodyagin, B. Casey, R. Partridge, "Luminosity Monitor Calibration", DØ internal note 4151.

[73] D. Krop, Ph.D. Thesis, Indiana University (2007).

[74] Ioana Anghel et al., "Studies of Taggability versus number of Primary Vertices for p17 data", DØ internal note 5240 .

[75] TMinuit, "http://root.cern/ch/root/html/TMinuit.html". 